NIST Special Publication 800-166

\title{
Derived PIV Application and Data Model Test Guidelines
}

\author{
David Cooper \\ Hildegard Ferraiolo \\ Ramaswamy Chandramouli \\ Nabil Ghadiali \\ Jason Mohler \\ Steven Brady
}

This publication is available free of charge from:

http://dx.doi.org/10.6028/NIST.SP.800-166

\section{COMPUTER SECUR I T Y}

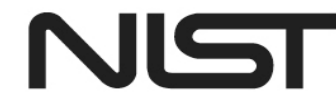

National Institute of Standards and Technology U.S. Department of Commerce 


\title{
NIST Special Publication 800-166
}

\section{Derived PIV Application and Data Model Test Guidelines}

\author{
David Cooper \\ Hildegard Ferraiolo \\ Ramaswamy Chandramouli \\ Computer Security Division \\ Information Technology Laboratory \\ Nabil Ghadiali \\ National Gallery of Art \\ Washington, $D C$ \\ Jason Mohler \\ Steven Brady \\ Electrosoft Services, Inc. \\ Reston, VA
}

This publication is available free of charge from

http://dx.doi.org/10.6028/NIST.SP.800-166

June 2016

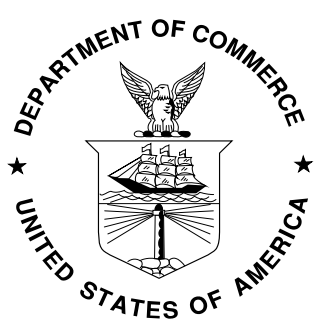

U.S. Department of Commerce Penny Pritzker, Secretary

National Institute of Standards and Technology Willie May, Under Secretary of Commerce for Standards and Technology and Director 


\section{Authority}

This publication has been developed by NIST in accordance with its statutory responsibilities under the Federal Information Security Modernization Act (FISMA) of 2014, 44 U.S.C. § 3541 et seq., Public Law (P.L.) 113-283. NIST is responsible for developing information security standards and guidelines, including minimum requirements for federal information systems, but such standards and guidelines shall not apply to national security systems without the express approval of appropriate federal officials exercising policy authority over such systems. This guideline is consistent with the requirements of the Office of Management and Budget (OMB) Circular A-130.

Nothing in this publication should be taken to contradict the standards and guidelines made mandatory and binding on Federal agencies by the Secretary of Commerce under statutory authority. Nor should these guidelines be interpreted as altering or superseding the existing authorities of the Secretary of Commerce, Director of the OMB, or any other Federal official. This publication may be used by nongovernmental organizations on a voluntary basis and is not subject to copyright in the United States. Attribution would, however, be appreciated by NIST.

\section{National Institute of Standards and Technology Special Publication 800-166 \\ Natl. Inst. Stand. Technol. Spec. Publ. 800-166, 143 pages (June 2016) \\ CODEN: NSPUE2}

This publication is available free of charge from:

http://dx.doi.org/10.6028/NIST.SP.800-166

Certain commercial entities, equipment, or materials may be identified in this document in order to describe an experimental procedure or concept adequately. Such identification is not intended to imply recommendation or endorsement by NIST, nor is it intended to imply that the entities, materials, or equipment are necessarily the best available for the purpose.

There may be references in this publication to other publications currently under development by NIST in accordance with its assigned statutory responsibilities. The information in this publication, including concepts and methodologies, may be used by federal agencies even before the completion of such companion publications. Thus, until each publication is completed, current requirements, guidelines, and procedures, where they exist, remain operative. For planning and transition purposes, federal agencies may wish to closely follow the development of these new publications by NIST.

Organizations are encouraged to review all draft publications during public comment periods and provide feedback to NIST. Many NIST cybersecurity publications, other than the ones noted above, are available at http://csrc.nist.gov/publications.

\section{Comments on this publication may be submitted to:}

National Institute of Standards and Technology

Attn: Computer Security Division, Information Technology Laboratory

100 Bureau Drive (Mail Stop 8930) Gaithersburg, MD 20899-8930

Email: pivtesting@nist.gov 


\title{
Reports on Computer Systems Technology
}

The Information Technology Laboratory (ITL) at the National Institute of Standards and Technology (NIST) promotes the U.S. economy and public welfare by providing technical leadership for the Nation's measurement and standards infrastructure. ITL develops tests, test methods, reference data, proof of concept implementations, and technical analyses to advance the development and productive use of information technology. ITL's responsibilities include the development of management, administrative, technical, and physical standards and guidelines for the cost-effective security and privacy of other than national security-related information in federal information systems. The Special Publication 800-series reports on ITL's research, guidelines, and outreach efforts in information system security, and its collaborative activities with industry, government, and academic organizations.

\begin{abstract}
NIST Special Publication (SP) 800-157 contains technical guidelines for the implementation of standards-based, secure, reliable, interoperable Public Key Infrastructure (PKI)-based identity credentials that are issued for mobile devices by federal departments and agencies to individuals who possess and prove control over a valid Personal Identity Verification (PIV) Card. This document, SP 800-166, contains the requirements and test assertions for testing the Derived PIV Application and associated Derived PIV data objects implemented on removable hardware tokens and within mobile devices. The tests reflect the design goals of interoperability and interface functions.
\end{abstract}

\section{Keywords}

authentication; derived PIV application; derived PIV application data model; derived PIV credential; derived test requirements (DTR); FIPS 201; implementation under test (IUT); mobile devices; Personal Identity Verification (PIV); test assertions; token command interface. 


\section{Acknowledgements}

The authors (David Cooper, Hildegard Ferraiolo and Ramaswamy Chandramouli of NIST; Nabil Ghadiali of the National Gallery of Art; and Jason Mohler and Steven Brady of Electrosoft Services, Inc.), wish to thank their colleagues who reviewed drafts of this document and contributed to its development. Special gratitude to the General Services Administration (GSA) FIPS 201 Evaluation Program (GSA FIPS 201 EP) team for their review and contributions to the document.

\section{Audience}

This document is targeted at vendors of Derived PIV Applications, issuers of Derived PIV Credentials, and entities that will conduct tests on these applications and credentials. Readers are assumed to have a working knowledge of SP 800-157, FIPS 201 and other PIV guidelines, and applicable technologies. This document is intended to:

+ Enable developers of Derived PIV Applications to design their applications as specified in SP 800-157 for interface, data object container size and access requirements.

+ Enable issuers of Derived PIV Credentials to ensure that Derived PIV data objects conform to the requirements specified in SP 800-157.

+ Enable developers and issuers to develop self-tests as part of the development effort and issuance process.

+ Enable entities performing conformance tests on Derived PIV Applications and Derived PIV data objects to develop tests that cover the test suite provided in this document. 


\section{Table of Contents}

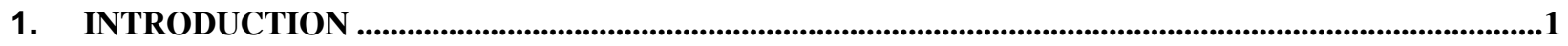

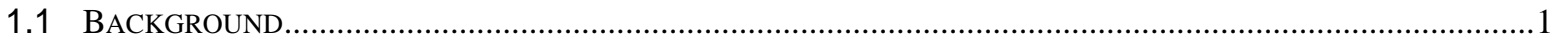

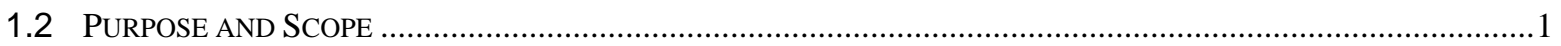

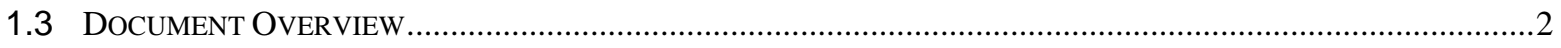

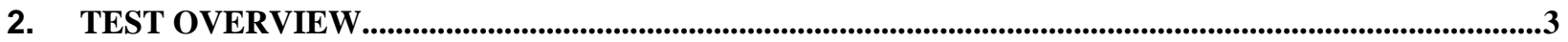

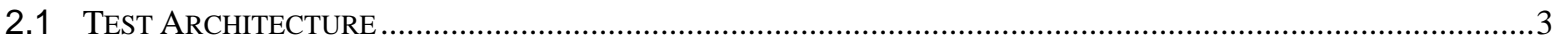

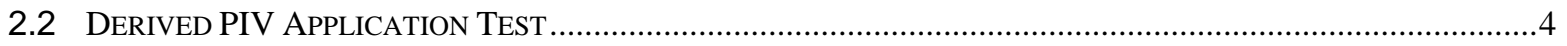

2.3 DATA Model Of THE DERIVEd PIV APPLICATION TESTS .............................................................................

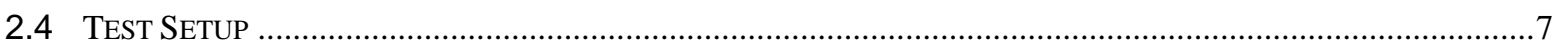

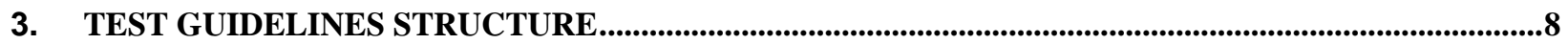

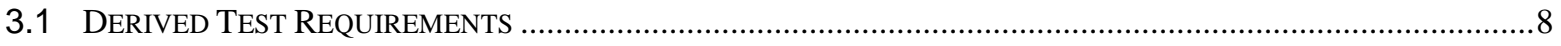

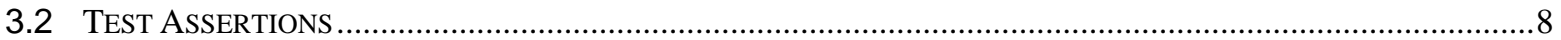

4. CONFORMANCE CRITERIA …......................................................................................................10

4.1 CONFORMANCE CRITERIA FOR DERIVED PIV APPLICATION ON REMOVABLE TOKENS ...............................10

4.2 Conformance CRITERIA For Data Model of THE DERIVEd PIV APPLICATION ...................................10

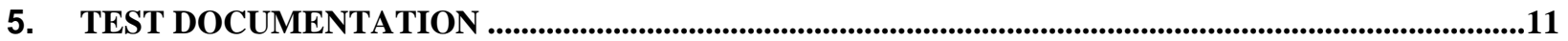

6. DERIVED TEST REQUIREMENTS FOR THE DERIVED PIV APPLICATION ON REMOVABLE

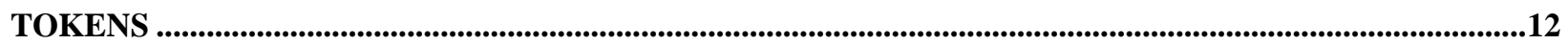

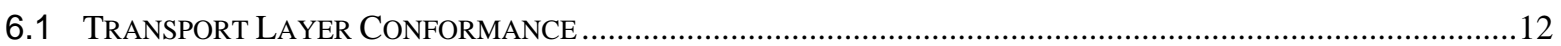

6.2 Derived PIV ApPLICATION DATA OBJECT ACCESS/StORAge CONFORMANCE …...................................13

6.3 DERIVED PIV APPLICATION COMMAND INTERFACE CONFORMANCE …….....................................................15

7. DERIVED TEST REQUIREMENTS FOR DATA MODEL OF THE DERIVED PIV APPLICATION 27

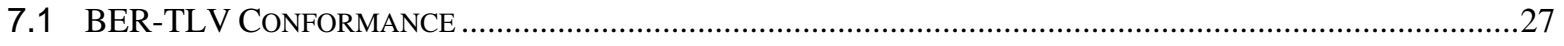

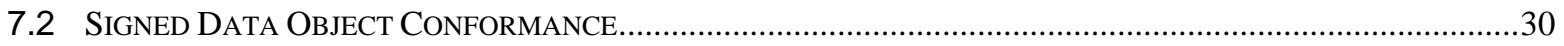

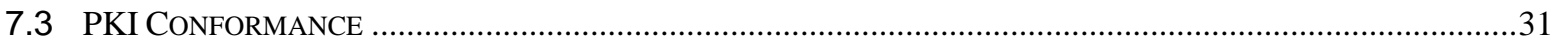

8. TEST ASSERTIONS FOR THE DERIVED PIV APPLICATION …...........................................................40

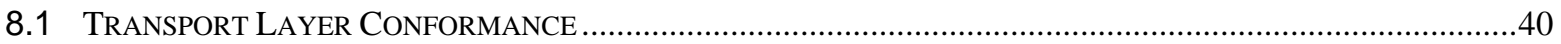

8.2 DeRIVED PIV APPLICATION DATA OBJECT ACCESS/STORAGE CONFORMANCE ...........................................41

8.3 DERIVED PIV APPLICATION COMMAND INTERFACE CONFORMANCE …….....................................................43

9. TEST ASSERTIONS FOR THE DERIVED PIV APPLICATION DATA MODEL .....................................88

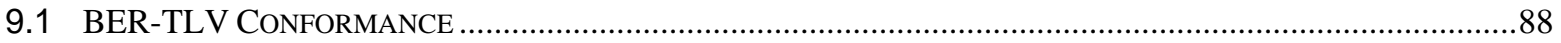

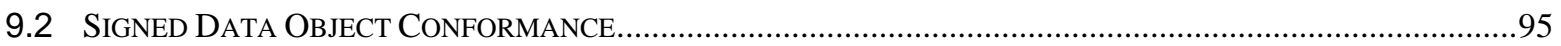

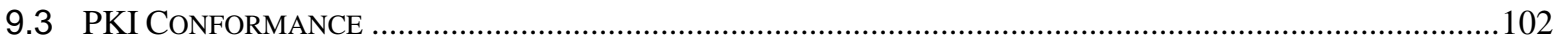




\section{List of Appendices}

APPENDIX A-TESTING OF DERIVED PIV CREDENTIALS ON EMBEDDED TOKENS .....................131

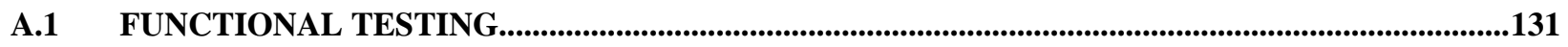

A.2 DATA MODEL TESTING ................................................................................................................131

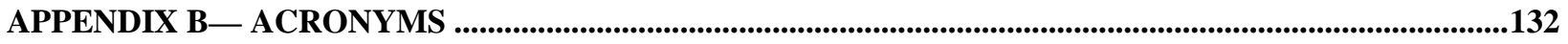

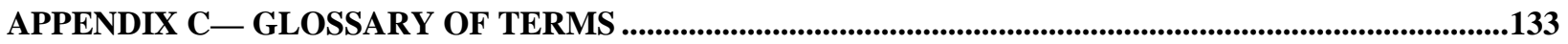

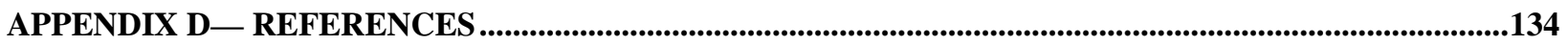

\section{List of Figures}

Figure 1 - DeRIVEd PIV ApPliCATION CONFORMANCE TEST ARCHITECTURE ............................. 3

\section{List of Tables}

TABLE 1 - ENCODING OF LENGTH FIELD 


\section{Introduction}

\subsection{Background}

Federal Information Processing Standard (FIPS) 201, Personal Identity Verification (PIV) for Federal Employees and Contractors [FIPS201], specified a common set of identity credentials for the purpose of Homeland Security Presidential Directive 12 [HSPD12] in a smart card form factor, known as the PIV Card. [FIPS201] originally required that all PIV credentials and associated keys be stored on the PIV Card, and although the use of the PIV Card for electronic authentication works well with traditional desktop and laptop computers, it is not optimized for mobile devices. ${ }^{1}$

In response to the growing use of mobile devices within the Federal Government, [FIPS201] was revised to permit the issuance of an additional credential specifically for mobile devices. This PIV credential is called a Derived PIV Credential, for which the corresponding private key is stored in a cryptographic module within a mobile device. The use of this Derived PIV Credential is restricted to provide PIV-enabled authentication services on mobile devices in order to authenticate the credential holder to remote systems.

\subsection{Purpose and Scope}

The objective of this document is to provide test requirements and test assertions that could be used to validate the compliance/conformance of the following: (i) the Derived PIV Application and (ii) the Derived PIV data model. Because NIST Special Publication (SP) 800-157, Guidelines for Derived Personal Identity Verification (PIV) Credentials [SP800-157], was developed for meeting interoperability goals of [FIPS201], the conformance tests in this document provide the assurance that the Derived PIV Application and associated derived PIV data objects that have passed these tests are conformant to the specification. This in turn facilitates procurement of [FIPS201]-products that are interoperable and meet the goals of [HSPD12].

[SP800-157] specifies the use of removable tokens with form factors that may be inserted into mobile devices, such as Secure Digital (SD) Cards, Universal Service Bus (USB) tokens, Universal Integrated Circuit Cards (UICC - the new generation of Subscriber Identity Module (SIM) cards), and non-removable tokens that are embedded in mobile devices. [SP800-157] does not define an application interface for embedded tokens, because these tokens are built into the mobile device and the interface to these tokens is natively supported. Since [SP800-157] does not specify application interface requirements for embedded tokens, testing the interfaces for embedded tokens is outside the scope of this document. ${ }^{2}$ In addition, this document does not

\footnotetext{
${ }^{1}$ A mobile device "is a portable computing device that: (i) has a small form factor such that it can easily be carried by a single individual; (ii) is designed to operate without a physical connection (e.g., wirelessly transmit or receive information); (iii) possesses local, non-removable or removable data storage; and (iv) includes a self-contained power source. Mobile devices may also include voice communication capabilities, on-board sensors that allow the devices to capture information, and/or built-in features for synchronizing local data with remote locations. Examples include smart phones, tablets, and e-readers.” [SP800-157, p. iv]

${ }^{2}$ Guidelines on functional and data model testing for embedded tokens are covered in Appendix A.
} 
provide conformance tests for any other software, such as the back-end access control software, issuance software, or any specialized service provider software used for logical access.

\subsection{Document Overview}

The document is organized as follows:

+ Section 2 provides a conceptual overview of the test architecture, the test setup and components, and the types of tests (Derived PIV Application and Data Model of the Derived PIV Application) covered within this document.

+ Section 3 describes the structure of the test guidelines and explains Derived test requirements (DTR) and test assertions (TA) construction.

$+\underline{\text { Section } 4}$ details the conformance criteria for each type of test.

$+\quad$ Section 5 explains the documentation necessary to conduct testing.

+ Section 6 includes DTRs that apply to the Derived PIV Application based on specifications in [SP800-157].

+ Section 7 includes DTRs that apply to the Data Model of the Derived PIV Application based on specifications in [SP800-157].

+ Section 8 provides test assertions that are used to test the DTRs of the Derived PIV Application listed in Section 6.

+ Section 9 provides test assertions that are used to test the DTRs of the Derived PIV Application data model listed in Section 7.

+ Appendix A contains guidelines for functional and data model testing of Derived PIV Credentials on embedded (non-removable) tokens.

+ Appendix B contains a list of acronyms used in the document.

+ Appendix C contains a glossary of terms used in the document.

+ Appendix D contains the list of documents used as references by this document. 


\section{Test Overview}

\subsection{Test Architecture}

SP 800-166 covers the following two types of tests for removable tokens: (i) Derived PIV Application and (ii) Data Model of the Derived PIV Application. The conceptual architecture for these tests is highlighted with dashed lines and shown in Figure 1.

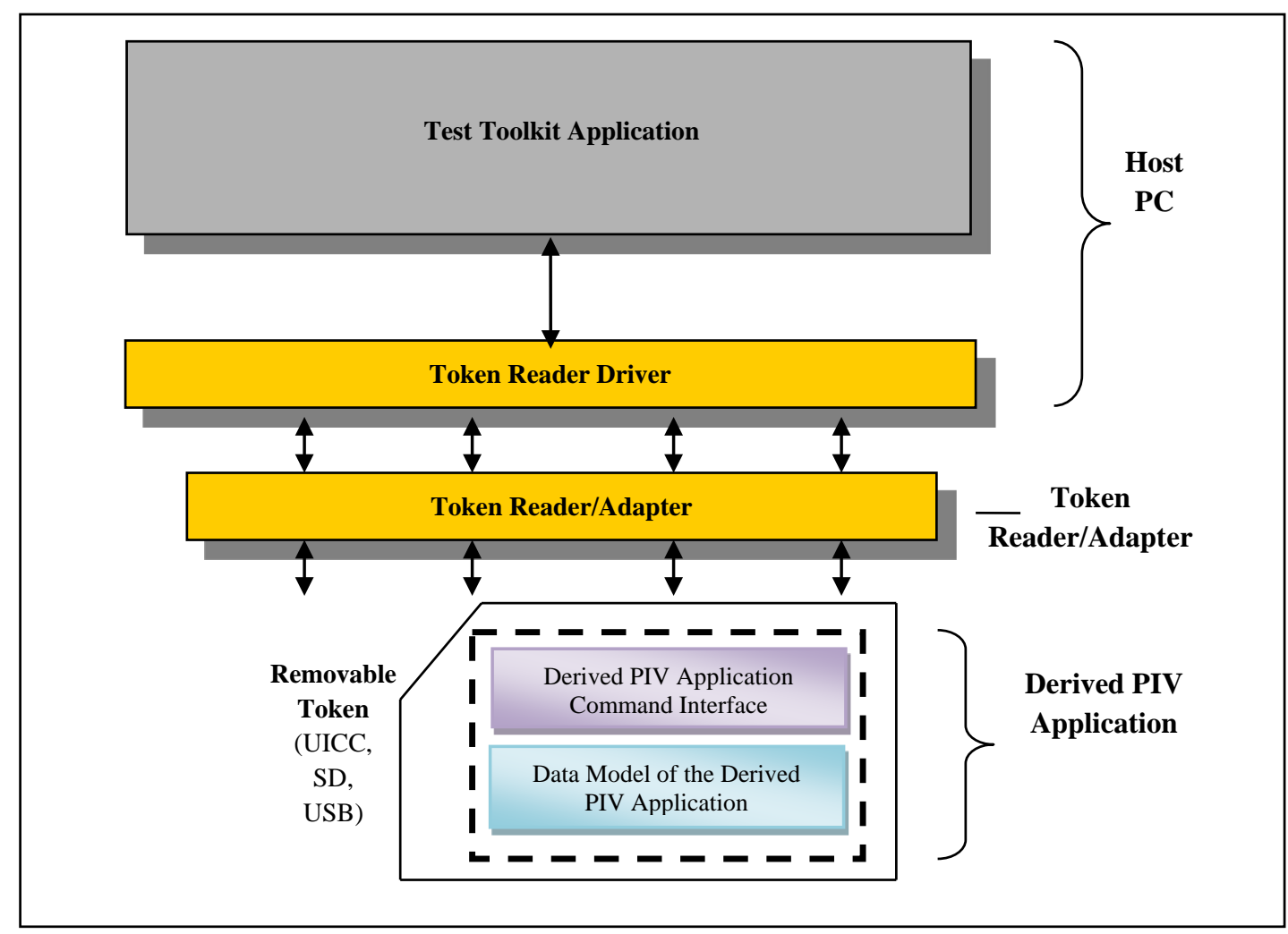

Figure 1 - Derived PIV Application Conformance Test Architecture

The Derived PIV Application resides on the removable hardware cryptographic token, ${ }^{3}$ implements the commands in the Derived PIV Application command interface, ${ }^{4}$ and provides access to data objects on the Derived PIV Application.

Given that [SP800-157] does not specify an application interface or an explicit data model for embedded tokens, vendors may implement Derived PIV Credentials on these devices in a manner of their choosing. Test entities may develop test assertions to test Derived PIV Credentials implemented on such tokens using functional testing developed specifically for the environment and application that they are being used within. Appendix A provides guidelines on testing Derived PIV Credentials (i.e., Derived PIV Authentication certificates) as well as other

\footnotetext{
3 "Token" in this context refers to the secure element that contains the Derived PIV Application.

${ }^{4}$ The Derived PIV Application command interface is as defined in [SP800-157], Appendix B.2 - Derived PIV Application Token Command Interface.
} 
certificates (digital signature certificate, key management certificates, etc.) that may be stored on embedded tokens.

\subsection{Derived PIV Application Test}

These tests are intended to ensure that tokens with Derived PIV Applications, sold and supplied by vendors, conform to the requirements specified in SP 800-157. In general, these tests cover the following:

+ Transport layer conformance, which ensures interoperability and portability of the Derived PIV Application token between mobile devices.

+ The Derived PIV Application data object access/storage conformance, which ensures that the application is set up and is conformant to [SP800-157] with regards to data object container sizes, data object identifiers, password requirements as well as the security conditions for accessing and storing each of the associated data objects.

+ The Derived PIV Application command interface as per [SP800-157], and includes the security conditions for executing each command in the interface with appropriate response statuses.

The tests should be performed via test scripts that communicate directly with the hardware cryptographic token through the API of the associated reader drivers and includes the following categories of tests.

\subsubsection{Transport Layer Conformance}

Transport layer conformance tests ensure that an implementation on a specific removable hardware token (i.e., UICC or USB) is compliant with industry standards specified in [SP800157] and that portability of the token is achieved across mobile devices.

\subsubsection{Derived PIV Application Data Object Access/Storage Conformance}

The Derived PIV Application data object access/storage conformance tests ensure that the Derived PIV Application is set up and configured per the requirements specified in [SP800-157]. It covers requirements that apply to the removable hardware cryptographic token and includes testing that covers containers for the following data objects:

+ The one mandatory data object as defined in [SP800-157]:

- X.509 Certificate for Derived PIV Authentication.

+ The twenty-five optional data objects, defined in [SP800-157]:

- X.509 Certificate for Digital Signature,

- X.509 Certificate for Key Management,

- Discovery Object, 
- Key History Object,

- 20 Retired X.509 Certificates for Key Management, and

- Security Object.

The containers will be validated for the following conditions:

+ Presence of containers for the mandatory data object and all supported optional data objects as specified in the vendor documentation;

+ Accessibility and storage of data objects using the appropriate BER-TLV tags (specified identifiers as per Section 4, Part 1 of NIST SP 800-73, Interfaces for Personal Identity Verification [SP800-73]);

+ Appropriate container size allocations for each of the data objects;

$+\quad$ Data objects access rule (password vs. no password); and

+ Security condition for data objects access/storage (cryptographic authentication).

\subsubsection{Derived PIV Application Command Interface Conformance}

These tests will validate that the implementation under test can successfully execute the commands in the Derived PIV Application token command interface as mandated by [SP800157]. Successful execution constitutes the Derived PIV Application responding with appropriate data and response status words to the commands sent by a test system. It also involves setting state variables per the specification. For example, the criteria for successful execution of the SELECT command involve the following:

+ The response status word returned is '90 00'.

+ The application property template is returned with the correct format and content.

+ The "Derived PIV Application” is the value of "currently selected application" (state variable)

The Derived PIV Application token command interface test suite includes conformance tests for the following commands:

+ Data access commands-

- SELECT

- GET DATA

+ Authentication commands-

- GENERAL AUTHENTICATE 
- VERIFY

- CHANGE REFERENCE DATA

- RESET RETRY COUNTER

+ Credential initialization and administration commands-

- PUT DATA

- GENERATE ASYMMETRIC KEY PAIR.

The token commands will be validated against the following conditions:

+ Precondition for use (password, cryptographic authentication),

+ Expected response status word, and

+ Appropriate state variables set in the Derived PIV Application.

\subsection{Data Model of the Derived PIV Application Tests}

These tests are intended to ensure that issuers populate the containers within the Derived PIV Application with data objects that conform to [SP800-157], [SP800-73] and [SP800-78]. In general, these tests cover the following:

+ Data objects are formatted correctly,

+ Field values are in accordance with the specifications, and

+ Data consistency and value computations such as signatures are accurate.

The tests should be performed via test scripts that communicate directly with the hardware cryptographic token through the API of the associated reader drivers and includes the following categories of tests.

\subsubsection{BER-TLV Format Conformance}

These tests validate that the tags and lengths of various data objects conform to specifications in [SP800-157].

\subsubsection{Signed Data Object Conformance}

For the Security Object, the tests check to ensure that the fields in the signature block conform to the Cryptographic Message Syntax (CMS). 


\subsubsection{PKI Conformance}

The Public Key Infrastructure (PKI) conformance tests ensure that the mandatory Derived PIV Authentication certificate, the optional digital signature certificate, key management certificates, and the Derived PIV Credential Issuer's (content signing) certificate, conform to the certificate profiles as specified in the X.509 Certificate and Certificate Revocation List (CRL) Extensions Profile for the Shared Service Providers (SSP) Program [PROF]. Additionally, the Derived PIV Application is also tested to check if asymmetric keys are pairs (public and private components) and are using the appropriate cryptographic algorithms in accordance with NIST SP 800-78, Cryptographic Algorithms and Key Sizes for Personal Identity Verification [SP800-78].

\subsection{Test Setup}

The test setup for the application and data model tests for the Derived PIV Application consists of the following components:

+ Test toolkit application software that resides on a personal computer (PC) with a Universal Serial Bus (USB) port;

+ Readers and appropriate drivers:

o For implementations on an SD, MiniSD or microSD token ${ }^{5}$

- A Secure Digital (SD) Memory Card Reader

- A Memory Card Reader Adapter (for Mini, and Micro SD Cards)

o For implementation on an UICC token-

- A PC/SC compliant UICC/SIM Card Reader

o For implementations on micro USB-

- A Universal Micro USB to USB Cable;

+ The implementation under test (IUT), which could be either:

- A Derived PIV Application, or

- The data object stored on a Derived PIV Application token. ${ }^{6}$

\footnotetext{
${ }^{5}$ [SP800-157] does not specify a transport layer protocol for SD, miniSD or microSD tokens. Since all implementations have to be tested from a common test toolkit application, vendors may be asked to provide a wrapper driver/middleware that utilizes a standard interface such as PC/SC to ensure that all tests can be performed without any modification or custom code development by the testing entities.

${ }^{6}$ Individual credentials (e.g., Derived PIV Authentication certificate, digital signature certificate, key management certificate) and associated private keys on embedded tokens may also be tested for conformity using functional testing. Refer to Appendix A for details.
} 


\section{Test Guidelines Structure}

\subsection{Derived Test Requirements}

Derived test requirements (DTRs) identify conformity conditions based on the normative specifications in [SP800-157] and any other referenced supporting publications (e.g., [FIPS201], [SP800-73], [SP800-78]).

In general, each DTR consists of the following elements:

1. An Identifier, which is a code starting with 'DTR' (Identifiers follow a running sequence based on a logical grouping of requirements);

2. A DTR Description, which is a statement taken/derived from the specification. These DTR descriptions include explicit statements using the words "shall," "must," and other terms used to signify the importance of the requirement; and

3. For traceability, each DTR includes a reference to the section of [SP800-157], any other applicable specification such as [FIPS201], [SP800-73], [SP800-78] or [PROF] from which the DTR has been taken.

\subsection{Test Assertions}

A test assertion is an action or a set of actions that is performed to measure conformity to one or more DTRs. Test assertions provide procedures to guide the tester in executing and managing the test.

In general, each test assertion consists of the following elements:

1. An Identifier, which is a code starting with 'TA' (Identifiers follow a running sequence based on categories of tests);

2. The Purpose of the test;

3. For the purpose of traceability, each test assertion makes specific references to the related DTR(s). Overall there is a many-to-many relationship between test assertions and DTRs (i.e., one test assertion can map to many DTRs and one DTR can be mapped to many test assertions);

4. Vendor/Issuer Documentation, which specifies the information that is needed in order to be able to execute the test. In general, vendors submit documentation for testing the Derived PIV Application while issuers submit documentation for testing the Data Model of the Derived PIV Application;

5. Precondition(s), which describe starting conditions and any prerequisites;

6. Test Scenario, which explains the test procedure in steps; 
7. Expected Result, which specifies the success criteria; and

8. Postcondition(s), which describes the final state after completion of the test scenario.

The test assertions for some of the DTRs do not have test scenarios. In such cases, documentation and/or test artifacts may be reviewed to determine compliance with the DTR. 


\section{Conformance Criteria}

Conformance criteria are based on the compliance of the "conformity condition" under the test with the requirements defined in [SP800-157] or any other referenced special publication (e.g., [FIPS201], [SP800-73], [SP800-78]) The criterion for success for each test assertion is based on the type of test being conducted.

\subsection{Conformance Criteria for Derived PIV Application on Removable Tokens}

The Derived PIV Application tests validate conformance to [SP800-157] of a Derived PIV Application developed by a vendor. The criterion for success is documented as part of the expected result or the required vendor documentation for each test assertion. Overall conformance of a removable token's Derived PIV Application is based on passing the following three categories of tests:

1. Transport layer conformance tests,

2. Derived PIV Application data object access/storage conformance tests, and

3. Derived PIV Application command interface conformance tests.

\subsection{Conformance Criteria for Data Model of the Derived PIV Application}

The data model tests validate the data objects that are loaded onto a conformant Derived PIV Application's removable token by an issuer. The criterion for success is documented as part of the expected result or the required issuer documentation for each test assertion. Overall conformance of the data model of the Derived PIV Application is based on passing the following three categories of tests:

1. BER-TLV format conformance tests,

2. Signed Data Object conformance tests, and

3. PKI conformance tests.

Testing entities may also validate individual credentials (e.g., Derived PIV Authentication certificate, digital signature certificate, key management certificate) stored on embedded (nonremovable) tokens. As described in Appendix A, in many cases it will also be possible to perform functional testing of the corresponding private keys. 


\section{Test Documentation}

There are two sets of documentation that are part of the compliance testing process:

vendor/issuer provided and testing entity generated. These documentations apply to both: (i) Derived PIV Application tests and (ii) Data Model of the Derived PIV Application tests.

The vendor/issuer documentation consists of the following:

+ Technical documentation: Technical details for the Derived PIV Application and its data model (as implemented). It includes, at a minimum, all the required information necessary to meet individual test assertions as documented in Section 8 (Test Assertions for the Derived PIV Application) or Section 9 (Test Assertions for the Data Model of the Derived PIV Application) of this document, depending on which tests are being performed; and

+ Security-related information: (a) Derived PIV Application Password, (b) Password Unblocking Key (PUK), (c) cryptographic algorithms supported by the application, and (d) the number of unsuccessful attempts using: (i) wrong Derived PIV Application Password and (ii) wrong PUK.

The testing entity documents are generated during testing and report test results. They include:

+ Test logs: A test log is kept for each test run on any component and is used to summarize the results of all the tests run; and

+ Test reports: These provide the background (environmental information) for each of the test assertions as well as summary of outcomes from test runs (from test logs) associated with each test assertion. 


\section{Derived Test Requirements for the Derived PIV Application on Removable} Tokens

This section lists requirements that apply to a Derived PIV Application resident on a removable token, such as an SD card, USB token, or UICC, that may be inserted into mobile devices. The requirements are aimed towards vendors of Derived PIV Applications to ensure that these applications are implemented correctly and are in accordance to the specification.

\subsection{Transport Layer Conformance ${ }^{7}$}

\subsubsection{UICC}

\begin{tabular}{|c|c|c|}
\hline DTR No: & DTR Description & Spec. Reference \\
\hline DTR-06.01.01.01 & $\begin{array}{l}\text { For a Universal Integrated Circuit Card (UICC) } \\
\text { used to host a Derived PIV Application, the } \\
\text { UICC shall implement the GlobalPlatform Card } \\
\text { Secure Element Configuration v1.0 [GPSE]. }\end{array}$ & $\begin{array}{l}\text { [SP800-157], } \\
\text { Section 3.3.1.2 - } \\
\text { Removable UICC } \\
\text { with Cryptographic } \\
\text { Module }\end{array}$ \\
\hline
\end{tabular}

\subsubsection{USB}

\begin{tabular}{|c|c|c|}
\hline DTR No: & DTR Description & Spec. Reference \\
\hline DTR-06.01.02.01 & $\begin{array}{l}\text { For a USB Integrated Circuit(s) Card Device } \\
\text { (ICCD) used to host a Derived PIV } \\
\text { Application, the ICCD shall comply with the } \\
\text { Universal Serial Bus Device Class - Smart } \\
\text { Card ICCD Specification for USB Integrated } \\
\text { Circuit(s) Card Devices [ICCDSPEC]. }\end{array}$ & $\begin{array}{l}\text { [SP800-157], } \\
\text { Section 3.3.1.3 - } \\
\text { USB Token with } \\
\text { Cryptographic } \\
\text { Module }\end{array}$ \\
\hline DTR-06.01.02.02 & $\begin{array}{l}\text { USB tokens with cryptographic modules that } \\
\text { support a Derived PIV Application shall be } \\
\text { compliant with the specifications in SP 800-96, } \\
\text { PIV Card to Reader Interoperability } \\
\text { Guidelines [SP800-96], for Application } \\
\text { Protocol Data Unit (APDU) support for contact } \\
\text { card readers. }\end{array}$ & $\begin{array}{l}\text { [SP800-157], } \\
\text { Section 3.3.1.3 - } \\
\text { USB Token with } \\
\text { Cryptographic } \\
\text { Module }\end{array}$ \\
\hline DTR-06.01.02.03 & $\begin{array}{l}\text { The APDUs for the Derived PIV Application } \\
\text { (as specified in Appendix B of [SP800-157]) } \\
\text { shall be transported to the secure element using } \\
\text { the Bulk-Out command pipe and the responses } \\
\text { shall be received from the secure element } \\
\text { using the Bulk-In command pipe. }\end{array}$ & $\begin{array}{l}\text { [SP800-157], } \\
\text { Section 3.3.1.3 - } \\
\text { USB Token with } \\
\text { Cryptographic } \\
\text { Module }\end{array}$ \\
\hline
\end{tabular}

\footnotetext{
${ }^{7}$ This document does not include test requirements for the SD card transport layer, since there are no requirements specified in [SP800-157].
} 


\subsection{Derived PIV Application Data Object Access/Storage Conformance}

\subsubsection{General}

\begin{tabular}{|c|c|c|}
\hline DTR No: & DTR Description & Spec. Reference \\
\hline DTR-06.02.01.01 & $\begin{array}{l}\text { The Derived PIV Application shall only } \\
\text { support a contact interface. }\end{array}$ & $\begin{array}{l}\text { [SP800-157], } \\
\text { Appendix B.1.2.1 - } \\
\text { Derived PIV } \\
\text { Application Data } \\
\text { Object Containers } \\
\text { and associated } \\
\text { Access Rules }\end{array}$ \\
\hline DTR-06.02.01.02 & $\begin{array}{l}\text { There shall be at most one Derived PIV } \\
\text { Application on any hardware cryptographic } \\
\text { token. }\end{array}$ & $\begin{array}{ll}\text { - } & \text { [SP800-157], } \\
& \text { Appendix B.2 - } \\
& \text { Derived PIV } \\
& \text { Application Token } \\
& \text { Command Interface } \\
\text { - } & \text { [SP800-73], Part 2, } \\
& \text { Section 3.1.1 - } \\
\text { SELECT Card } \\
\text { Command }\end{array}$ \\
\hline DTR-06.02.01.03 & $\begin{array}{l}\text { The AID of the Derived Personal Identity } \\
\text { Verification Application shall be: 'A0 } 000003 \\
08000020000100 \text { '. }\end{array}$ & $\begin{array}{l}\text { - } \text { [SP800-157], } \\
\text { Appendix B.1.1 - } \\
\text { Derived PIV } \\
\text { Application } \\
\text { Identifier }\end{array}$ \\
\hline
\end{tabular}

\subsubsection{Derived PIV Application Data Objects and Representation}

\begin{tabular}{|c|c|c|}
\hline DTR No: & DTR Description & Spec. Reference \\
\hline DTR-06.02.02.01 & $\begin{array}{l}\text { The Derived PIV Application shall contain an } \\
\text { X.509 Certificate for Derived PIV } \\
\text { Authentication container and optionally the } \\
\text { following containers: (i) X.509 Certificate for } \\
\text { Digital Signature, (ii) X.509 Certificate for Key } \\
\text { Management, (iii) Discovery Object, (iv) Key } \\
\text { History Object, (v) up to } 20 \text { Retired X.509 } \\
\text { Certificates for Key Management and (vi) } \\
\text { Security Object. }\end{array}$ & $\begin{array}{l}\text { - [SP800-157], } \\
\text { Appendix B.1.2 - } \\
\text { Derived PIV } \\
\text { Application Data } \\
\text { Model Elements } \\
\text { - [SP800-73], Part 1, } \\
\text { Appendix A - PIV } \\
\text { Data Model, Table 10 }\end{array}$ \\
\hline DTR-06.02.02.02 & $\begin{array}{l}\text { The minimum capacity for the X. } 509 \\
\text { Certificate for Derived PIV Authentication } \\
\text { shall be } 1905 \text { bytes. }\end{array}$ & $\begin{array}{l}\text { [SP800-73], Part 1, } \\
\text { Appendix A - PIV } \\
\text { Data Model, Table } 7\end{array}$ \\
\hline
\end{tabular}




\begin{tabular}{|c|c|c|}
\hline DTR No: & DTR Description & Spec. Reference \\
\hline DTR-06.02.02.03 & $\begin{array}{l}\text { The minimum capacity for the X.509 } \\
\text { Certificate for Digital Signature shall be } 1905 \\
\text { bytes. }\end{array}$ & $\begin{array}{l}\text { - } \text { [SP800-73], Part 1, } \\
\text { Appendix A - PIV } \\
\text { Data Model, Table } 7\end{array}$ \\
\hline DTR-06.02.02.04 & $\begin{array}{l}\text { The minimum capacity for the X.509 } \\
\text { Certificate for Key Management shall be } 1905 \\
\text { bytes. }\end{array}$ & $\begin{array}{l}\text { [SP800-73], Part 1, } \\
\text { Appendix A - PIV } \\
\text { Data Model, Table } 7\end{array}$ \\
\hline DTR-06.02.02.05 & $\begin{array}{l}\text { The minimum capacity for the Discovery } \\
\text { Object shall be } 19 \text { bytes. }\end{array}$ & $\begin{array}{l}\text { - } \text { [SP800-73], Part 1, } \\
\text { Appendix A - PIV } \\
\text { Data Model, Table } 7\end{array}$ \\
\hline DTR-06.02.02.06 & $\begin{array}{l}\text { The minimum capacity for the Key History } \\
\text { Object shall be } 128 \text { bytes. }\end{array}$ & $\begin{array}{l}\text { - } \text { [SP800-73], Part 1, } \\
\text { Appendix A - PIV } \\
\text { Data Model, Table } 7\end{array}$ \\
\hline DTR-06.02.02.07 & $\begin{array}{l}\text { The minimum capacity for each Retired X.509 } \\
\text { Certificate for Key Management shall be } 1905 \\
\text { bytes. }\end{array}$ & $\begin{array}{l}\text { - } \text { [SP800-73], Part 1, } \\
\text { Appendix A - PIV } \\
\text { Data Model, Table } 7\end{array}$ \\
\hline DTR-06.02.02.08 & $\begin{array}{l}\text { The minimum capacity for the Security Object } \\
\text { container shall be } 3000 \text { bytes. }\end{array}$ & $\begin{array}{l}\text { - [SP800-157], } \\
\text { Appendix B.1.2.1 - } \\
\text { Derived PIV } \\
\text { Application Data } \\
\text { Object Containers } \\
\text { and associated } \\
\text { Access Rules }\end{array}$ \\
\hline DTR-06.02.02.09 & $\begin{array}{l}\text { The status words that may be returned on the } \\
\text { Derived PIV Application command interface } \\
\text { are as specified in Section } 5.6 \text { of [SP800-73], } \\
\text { Part } 1 .\end{array}$ & $\begin{array}{l}\text { - [SP800-157], } \\
\text { Appendix B.4.3 - } \\
\text { Derived PIV } \\
\text { Application Status } \\
\text { Words }\end{array}$ \\
\hline DTR-06.02.02.10 & $\begin{array}{l}\text { "Basic encoding rules - tag length value" } \\
\text { (BER-TLV) tags for the various mandatory and } \\
\text { optional data objects within the Derived PIV } \\
\text { Application are the same as for the } \\
\text { corresponding data objects (mapped as per the } \\
\text { Table B-1 of [SP800-157]) of the PIV Card } \\
\text { Application as described in Section } 4 \text { of } \\
\text { [SP800-73], Part } 1 \text {. }\end{array}$ & $\begin{array}{l}\text { - [SP800-157], } \\
\text { Appendix B.1.3 - } \\
\text { Derived PIV } \\
\text { Application Data } \\
\text { Objects } \\
\text { Representation }\end{array}$ \\
\hline DTR-06.02.02.11 & $\begin{array}{l}\text { Key reference values used on the Derived PIV } \\
\text { Application interfaces shall be in accordance } \\
\text { with Table 6-1 of [SP800-78] and Table 4a and } \\
\text { Table 4b of [SP800-73], Part } 1 \text { with the } \\
\text { mappings defined in Table B-2 of [SP800-157]. }\end{array}$ & $\begin{array}{l}\text { - [SP800-157], } \\
\text { Appendix B.1.4.1 - } \\
\text { Derived PIV } \\
\text { Application Key } \\
\text { References and } \\
\text { Security Conditions } \\
\text { of Use }\end{array}$ \\
\hline
\end{tabular}




\begin{tabular}{|c|c|c|}
\hline DTR No: & DTR Description & Spec. Reference \\
\hline DTR-06.02.02.12 & $\begin{array}{l}\text { The algorithm identifiers for the cryptographic } \\
\text { algorithms that may be recognized on the } \\
\text { Derived PIV Application interfaces are the } \\
\text { asymmetric and symmetric identifiers specified } \\
\text { in Table } 6-2 \text { and Table } 6-3 \text { of [SP800-78]. The } \\
\text { cryptographic mechanism identifiers that may } \\
\text { be recognized on the Derived PIV Application } \\
\text { interfaces are those specified in Table } 5 \text { of } \\
\text { [SP800-73], Part } 1 \text {. }\end{array}$ & $\begin{array}{l}\text { - [SP800-157], } \\
\text { Appendix B.1.4.2 - } \\
\text { Derived PIV } \\
\text { Application } \\
\text { Cryptographic } \\
\text { Algorithm and } \\
\text { Mechanism } \\
\text { Identifiers }\end{array}$ \\
\hline DTR-06.02.02.13 & $\begin{array}{l}\text { The Derived PIV Application Password shall } \\
\text { be between } 6 \text { and } 8 \text { bytes in length. The } \\
\text { Derived PIV Application shall enforce the } \\
\text { minimum length requirement of six bytes for } \\
\text { the Derived PIV Application Password (i.e., } \\
\text { shall verify that at least the first six bytes of the } \\
\text { value presented to the token command interface } \\
\text { are in the range [0x30-0x39, 0x41 - 0x5A, } \\
0 \times 61-0 \times 7 A]) \text {. }\end{array}$ & $\begin{array}{l}\text { - [SP800-157], } \\
\text { Appendix B.2.1 - } \\
\text { Authentication of an } \\
\text { Individual }\end{array}$ \\
\hline DTR-06.02.02.14 & $\begin{array}{l}\text { If the actual length of the Derived PIV } \\
\text { Application Password is less than } 8 \text { bytes, it } \\
\text { shall be padded to } 8 \text { bytes with 'FF' when } \\
\text { presented to the token command interface. The } \\
\text { 'FF' padding bytes shall be appended to the } \\
\text { actual value of the password. }\end{array}$ & $\begin{array}{l}\text { - [SP800-157], } \\
\text { Appendix B.2.1 - } \\
\text { Authentication of an } \\
\text { Individual }\end{array}$ \\
\hline DTR-06.02.02.15 & $\begin{array}{l}\text { The bytes comprising the Derived PIV } \\
\text { Application Password shall be limited to values } \\
0 \times 30-0 \times 39,0 x 41 \text { - 0x5A, and 0x61 - 0x7A, } \\
\text { the ASCII values for the decimal digits '0' - '9', } \\
\text { upper case characters 'A' - 'Z', and lower case } \\
\text { characters 'a' - 'z' respectively. }\end{array}$ & $\begin{array}{l}\text { - [SP800-157], } \\
\text { Appendix B.2.1 - } \\
\text { Authentication of an } \\
\text { Individual }\end{array}$ \\
\hline
\end{tabular}

\subsection{Derived PIV Application Command Interface Conformance}

\subsubsection{General}

\begin{tabular}{|l|l|l|}
\hline DTR No: & DTR Description & Spec. Reference \\
\hline DTR-06.03.01.01 & The command interface for the Derived PIV & $\bullet$ [SP800-157], \\
& Application shall implement all of the card & Appendix B.2 - \\
& commands supported by the PIV Card & Derived PIV \\
& Application as described in [SP800-73], Part 2, & Application Token \\
& which include: SELECT, GET DATA, & Command \\
& VERIFY, CHANGE REFERENCE DATA, & Interface \\
& RESET RETRY COUNTER, GENERAL & \\
& AUTHENTICATE, PUT DATA, GENERATE & \\
& ASYMMETRIC KEY PAIR. & \\
\hline
\end{tabular}




\begin{tabular}{|l|l|l|}
\hline DTR No: & DTR Description & Spec. Reference \\
\hline DTR-06.03.01.02 & $\begin{array}{l}\text { Token commands indicated with a 'Yes' in the } \\
\text { Command Chaining column in Table 2 of } \\
\text { [SP800-73], Part 2 shall support command } \\
\text { chaining for transmitting a data string too long } \\
\text { for a single command as defined in [ISO7816- } \\
\text { 4]. }\end{array}$ & $\begin{array}{l}\text { [SP800-73], Part 2, } \\
\text { Section 3 - PIV } \\
\text { Card Application } \\
\text { Interface }\end{array}$ \\
\hline
\end{tabular}

\subsubsection{SELECT Command}

\begin{tabular}{|c|c|c|}
\hline DTR No: & DTR Description & Spec. Reference \\
\hline DTR-06.03.02.01 & $\begin{array}{l}\text { The Derived PIV Application can be selected as } \\
\text { the current application on the removable } \\
\text { hardware cryptographic token by providing the } \\
\text { full AID as follows: 'A0 } 00000308000020 \\
000100 \text { '. }\end{array}$ & $\begin{array}{l}\text { [SP800-157], } \\
\text { Appendix B.1.1 - } \\
\text { Derived PIV } \\
\text { Application } \\
\text { Identifier }\end{array}$ \\
\hline DTR-06.03.02.02 & $\begin{array}{l}\text { The token platform shall support a default } \\
\text { selected application. In other words, there shall } \\
\text { be a currently selected application immediately } \\
\text { after a cold or warm reset. This application is } \\
\text { the default selected application. The default } \\
\text { application may be the Derived PIV } \\
\text { Application, or it may be another application. }\end{array}$ & $\begin{array}{l}\text { [SP800-157], } \\
\text { Appendix B.2 - } \\
\text { Derived PIV } \\
\text { Application Token } \\
\text { Command } \\
\text { Interface }\end{array}$ \\
\hline DTR-06.03.02.03 & $\begin{array}{l}\text { Upon selection, the Derived PIV Application } \\
\text { shall return the application property template } \\
\text { described in Table } 3 \text { of [SP800-73], Part 2, with } \\
\text { the exception that the returned AID is the AID } \\
\text { listed in Appendix B.1.1 of [SP800-157]. }\end{array}$ & $\begin{array}{l}\text { - } \text { [SP800-157], } \\
\text { Appendix B.2 - } \\
\text { Derived PIV } \\
\text { Application Token } \\
\text { Command } \\
\text { Interface } \\
\text { - } \\
\text { [SP800-73], Part 2, } \\
\text { Section 3.1.1 - } \\
\text { SELECT Card } \\
\text { Command }\end{array}$ \\
\hline DTR-06.03.02.04 & $\begin{array}{l}\text { The Derived PIV Application can also be made } \\
\text { the currently selected application by providing a } \\
\text { right-truncated version - that is, without the } \\
\text { two-byte version number, '01 00' - in the data } \\
\text { field of the SELECT command 'A0 } 00000308 \\
00002000 \text { ' }\end{array}$ & $\begin{array}{l}\text { [SP800-157], } \\
\text { Appendix B.2 - } \\
\text { Derived PIV } \\
\text { Application Token } \\
\text { Command } \\
\text { Interface } \\
\text { [SP800-73], Part 2, } \\
\text { Section 3.1.1 - } \\
\text { SELECT Card } \\
\text { Command }\end{array}$ \\
\hline
\end{tabular}




\begin{tabular}{|c|c|c|}
\hline DTR No: & DTR Description & Spec. Reference \\
\hline DTR-06.03.02.05 & $\begin{array}{l}\text { The complete AID, including the two-byte } \\
\text { version, of the Derived PIV Application that } \\
\text { became the currently selected application upon } \\
\text { successful execution of the SELECT command } \\
\text { (using the full or right-truncated PIV AID) shall } \\
\text { be returned in the application property template. }\end{array}$ & $\begin{array}{l}\text { [SP800-157], } \\
\text { Appendix B.2 - } \\
\text { Derived PIV } \\
\text { Application Token } \\
\text { Command } \\
\text { Interface } \\
\text { [SP800-73], Part 2, } \\
\text { Section 3.1.1 - } \\
\text { SELECT Card } \\
\text { Command }\end{array}$ \\
\hline DTR-06.03.02.06 & $\begin{array}{l}\text { If the currently selected application is the } \\
\text { Derived PIV Application when the SELECT } \\
\text { command is sent and the AID in the data field } \\
\text { of the SELECT command is either the AID of } \\
\text { the Derived PIV Application or its right- } \\
\text { truncated version thereof, then the Derived PIV } \\
\text { Application shall continue to be the currently } \\
\text { selected application and the setting of all } \\
\text { security status indicators in the Derived PIV } \\
\text { Application shall be unchanged. }\end{array}$ & $\begin{array}{l}\text { [SP800-157], } \\
\text { Appendix B.2 - } \\
\text { Derived PIV } \\
\text { Application Token } \\
\text { Command } \\
\text { Interface } \\
\text { [SP800-73], Part 2, } \\
\text { Section 3.1.1 - } \\
\text { SELECT Card } \\
\text { Command }\end{array}$ \\
\hline DTR-06.03.02.07 & $\begin{array}{l}\text { If the currently selected application is the } \\
\text { Derived PIV Application when the SELECT } \\
\text { command is sent and the AID in the data field } \\
\text { of the SELECT command is an invalid AID, } \\
\text { then the Derived PIV Application shall remain } \\
\text { the currently selected application and the } \\
\text { Derived PIV Application security status } \\
\text { indicator shall remain unchanged. }\end{array}$ & $\begin{array}{l}\text { [SP800-157], } \\
\text { Appendix B.2 - } \\
\text { Derived PIV } \\
\text { Application Token } \\
\text { Command } \\
\text { Interface } \\
\text { [SP800-73], Part 2, } \\
\text { Section 3.1.1 - } \\
\text { SELECT Card } \\
\text { Command }\end{array}$ \\
\hline DTR-06.03.02.08 & $\begin{array}{l}\text { If the currently selected application is the } \\
\text { Derived PIV Application when the SELECT } \\
\text { command is given and the AID in the data field } \\
\text { of the SELECT command is not the Derived } \\
\text { PIV Application (nor the right-truncated version } \\
\text { thereof), but a valid AID supported by the } \\
\text { token, then the Derived PIV Application shall } \\
\text { be deselected and the Derived PIV Application } \\
\text { security status indicators in the Derived PIV } \\
\text { Application shall be set to FALSE. }\end{array}$ & $\begin{array}{ll}\text { - } & \text { [SP800-157], } \\
\text { Appendix B.2 - } \\
\text { Derived PIV } \\
\text { Application Token } \\
\text { Command } \\
\text { Interface } \\
\text { - } \\
\text { [SP800-73], Part 2, } \\
\text { Section 3.1.1 - } \\
\text { SELECT Card } \\
\text { Command }\end{array}$ \\
\hline
\end{tabular}




\subsubsection{GET DATA Command}

\begin{tabular}{|c|c|c|}
\hline DTR No: & DTR Description & Spec. Reference \\
\hline DTR-06.03.03.01 & $\begin{array}{l}\text { The GET DATA command retrieves the data } \\
\text { content of the single data object whose tag is } \\
\text { given in the data field. }\end{array}$ & $\begin{array}{l}\text { - } \quad \text { SP800-157], } \\
\text { Appendix B.2 - } \\
\text { Derived PIV } \\
\text { Application Token } \\
\text { Command } \\
\text { Interface } \\
\text { - } \quad \text { [SP800-73], Part 2, } \\
\text { Section 3.1.2 - } \\
\text { GET DATA Card } \\
\text { Command }\end{array}$ \\
\hline DTR-06.03.03.02 & $\begin{array}{l}\text { The } L_{c} \text { value is '05' for all Derived PIV data } \\
\text { objects except for the } 0 x 7 E \text { interindustry tag } \\
\text { (Discovery Object), which has an Lc value of } \\
\text { '03'. }\end{array}$ & $\begin{array}{ll}\text { - } & \text { [SP800-157], } \\
\text { Appendix B.2 - } \\
\text { Derived PIV } \\
\text { Application Token } \\
\text { Command } \\
\text { Interface } \\
\text { - } \\
\text { [SP800-73], Part 2, } \\
\text { Section 3.1.2 - } \\
\text { GET DATA Card } \\
\text { Command }\end{array}$ \\
\hline DTR-06.03.03.03 & $\begin{array}{l}\text { The GET RESPONSE command is used in } \\
\text { conjunction with GET DATA to accomplish } \\
\text { the reading of larger Derived PIV data objects. }\end{array}$ & $\begin{array}{l}\text { - } \text { [SP800-73], Part 2, } \\
\text { Section 3.1.2 - } \\
\text { GET DATA Card } \\
\text { Command }\end{array}$ \\
\hline
\end{tabular}

\subsubsection{GENERAL AUTHENTICATE Command}

\begin{tabular}{|c|c|c|}
\hline DTR No: & DTR Description & Spec. Reference \\
\hline DTR-06.03.04.01 & $\begin{array}{l}\text { The GENERAL AUTHENTICATE command } \\
\text { shall be used with the Derived PIV } \\
\text { authentication keys ('9A' and '9B') using } \\
\text { cryptographic algorithms from Table 6-2 of } \\
\text { [SP800-78] to authenticate the token or a token } \\
\text { application to the client application } \\
\text { (INTERNAL AUTHENTICATE), to } \\
\text { authenticate an entity to the token } \\
\text { (EXTERNAL AUTHENTICATE), and to } \\
\text { perform a mutual authentication between the } \\
\text { token and an entity external to the token } \\
\text { (MUTUAL AUTHENTICATE). }\end{array}$ & $\begin{array}{ll} & \text { [SP800-157], } \\
\text { Appendix B.2 - } \\
\text { Derived PIV } \\
\text { Application Token } \\
\text { Command Interface } \\
\text { - } \quad \text { [SP800-73], Part 2, } \\
\text { Section 3.2.4 - } \\
\text { GENERAL } \\
\text { AUTHENTICATE } \\
\text { Card Command }\end{array}$ \\
\hline
\end{tabular}




\begin{tabular}{|c|c|c|}
\hline DTR No: & DTR Description & Spec. Reference \\
\hline DTR-06.03.04.02 & $\begin{array}{l}\text { The GENERAL AUTHENTICATE command } \\
\text { shall be used with the digital signature key } \\
\text { ('9C') (if implemented) to realize the signing } \\
\text { functionality on the Derived PIV Application } \\
\text { programming interface using cryptographic } \\
\text { algorithms specified in Table 3-1 of [SP800- } \\
\text { 78]. }\end{array}$ & $\begin{array}{ll}\text { - } & \text { [SP800-157], } \\
\text { Appendix B.2 - } \\
\text { Derived PIV } \\
\text { Application Token } \\
\text { Command Interface } \\
\text { - } \quad \text { [SP800-73], Part 2, } \\
\text { Section 3.2.4 - } \\
\text { GENERAL } \\
\text { AUTHENTICATE } \\
\text { Card Command }\end{array}$ \\
\hline DTR-06.03.04.03 & $\begin{array}{l}\text { The GENERAL AUTHENTICATE command } \\
\text { shall be used with the key management key } \\
\text { ('9D') (if implemented) and the retired key } \\
\text { management keys ('82' - '95') (if implemented) } \\
\text { to realize key establishment schemes specified } \\
\text { in [SP800-78] (ECDH and RSA). }\end{array}$ & $\begin{array}{ll}\text { - } & \text { [SP800-157], } \\
\text { Appendix B.2 - } \\
\text { Derived PIV } \\
\text { Application Token } \\
\text { Command Interface } \\
\text { - } \\
\text { [SP800-73], Part 2, } \\
\text { Section 3.2.4 - } \\
\text { GENERAL } \\
\text { AUTHENTICATE } \\
\text { Card Command }\end{array}$ \\
\hline DTR-06.03.04.04 & $\begin{array}{l}\text { The GENERAL AUTHENTICATE command } \\
\text { supports command chaining to permit the } \\
\text { uninterrupted transmission of long command } \\
\text { data fields to the Derived PIV Application. If a } \\
\text { token command other than the GENERAL } \\
\text { AUTHENTICATE command is received by } \\
\text { the Derived PIV Application before the } \\
\text { termination of a GENERAL } \\
\text { AUTHENTICATE chain, then the Derived } \\
\text { PIV Application shall rollback to the state it } \\
\text { was in immediately prior to the reception of the } \\
\text { first command in the interrupted chain. In other } \\
\text { words, an interrupted GENERAL } \\
\text { AUTHENTICATE chain has no effect on the } \\
\text { Derived PIV Application. }\end{array}$ & $\begin{array}{ll}\text { - } & \text { [SP800-157], } \\
\text { Appendix B.2 - } \\
\text { Derived PIV } \\
\text { Application Token } \\
\text { Command Interface } \\
\text { - } \quad \text { [SP800-73], Part 2, } \\
\text { Section 3.2.4 - } \\
\text { GENERAL } \\
\text { AUTHENTICATE } \\
\text { Card Command }\end{array}$ \\
\hline DTR-06.03.04.05 & $\begin{array}{l}\text { For cryptographic operations with larger keys, } \\
\text { e.g., RSA 2048, the GET RESPONSE } \\
\text { command is used to return the complete result } \\
\text { of the cryptographic operation. }\end{array}$ & $\begin{array}{ll}\text { - } & \text { [SP800-157], } \\
\text { Appendix B.2 - } \\
\text { Derived PIV } \\
\text { Application Token } \\
\text { Command Interface } \\
\text { - } \\
\text { [SP800-73], Part 2, } \\
\text { Section 3.2.4 - } \\
\text { GENERAL } \\
\end{array}$ \\
\hline
\end{tabular}




\begin{tabular}{|l|l|l|}
\hline DTR No: & DTR Description & Spec. Reference \\
\hline & & $\begin{array}{l}\text { AUTHENTICATE } \\
\text { Card Command }\end{array}$ \\
\hline
\end{tabular}

\subsubsection{VERIFY Command}

\begin{tabular}{|c|c|c|}
\hline DTR No: & DTR Description & Spec. Reference \\
\hline DTR-06.03.05.01 & $\begin{array}{l}\text { Key reference ' } 80 \text { ' shall be able to be verified by } \\
\text { the Derived PIV Application VERIFY } \\
\text { command. }\end{array}$ & $\begin{array}{l}\text { - } \quad \text { [SP800-157], } \\
\text { Appendix B.2 - } \\
\text { Derived PIV } \\
\text { Application Token } \\
\text { Command Interface } \\
\text { - } \quad \text { [SP800-73], Part 2, } \\
\text { Section 3.2.1 - } \\
\text { VERIFY Card } \\
\text { Command }\end{array}$ \\
\hline DTR-06.03.05.02 & $\begin{array}{l}\text { When the key reference is ' } 80 \text { ' and the current } \\
\text { value of the retry counter associated with the key } \\
\text { reference is zero, then the comparison shall not } \\
\text { be made, and the Derived PIV Application shall } \\
\text { return the status word '69 83'. }\end{array}$ & $\begin{array}{ll}\text { - } & \text { [SP800-157], } \\
\text { Appendix B.2 - } \\
\text { Derived PIV } \\
\text { Application Token } \\
\text { Command Interface } \\
\text { - } \quad \text { [SP800-73], Part 2, } \\
\text { Section 3.2.1 - } \\
\text { VERIFY Card } \\
\text { Command }\end{array}$ \\
\hline DTR-06.03.05.03 & $\begin{array}{l}\text { When the key reference is '80' and the } \\
\text { authentication data in the command data field } \\
\text { does not satisfy the criteria in Appendix B.2.1 of } \\
\text { [SP800-157], then the token command shall fail } \\
\text { and the Derived PIV Application shall return } \\
\text { either the status word '6A 80' or '63 CX'. If status } \\
\text { word '6A 80' is returned, the security status and } \\
\text { the retry counter of the key reference shall } \\
\text { remain unchanged. If status word '63 CX' is } \\
\text { returned, the security status of the key reference } \\
\text { shall be set to FALSE and the retry counter } \\
\text { associated with the key reference shall be } \\
\text { decremented by one. }\end{array}$ & $\begin{array}{ll}\text { - } & \text { [SP800-157], } \\
\text { Appendix B.2 - } \\
\text { Derived PIV } \\
\text { Application Token } \\
\text { Command Interface } \\
\text { - } \quad \text { [SP800-73], Part 2, } \\
\text { Section 3.2.1 - } \\
\text { VERIFY Card } \\
\text { Command }\end{array}$ \\
\hline DTR-06.03.05.04 & $\begin{array}{l}\text { When the key reference is ' } 80 \text { ' and the } \\
\text { authentication data in the command data field is } \\
\text { properly formatted and does not match reference } \\
\text { data associated with the key reference, then the } \\
\text { token command shall fail, the Derived PIV }\end{array}$ & $\begin{array}{l}\text { - } \text { [SP800-157], } \\
\text { Appendix B.2 - } \\
\text { Derived PIV } \\
\text { Application Token } \\
\text { Command Interface }\end{array}$ \\
\hline
\end{tabular}




\begin{tabular}{|c|c|c|}
\hline DTR No: & DTR Description & Spec. Reference \\
\hline & $\begin{array}{l}\text { Application shall return the status word ' } 63 \text { CX', } \\
\text { the security status of the key reference shall be } \\
\text { set to FALSE, and the retry counter associated } \\
\text { with the key reference (i.e., ' } 80 \text { ') shall be } \\
\text { decremented by one. }\end{array}$ & $\begin{array}{l}\text { [SP800-73], Part 2, } \\
\text { Section 3.2.1 - } \\
\text { VERIFY Card } \\
\text { Command }\end{array}$ \\
\hline DTR-06.03.05.05 & $\begin{array}{l}\text { If P1='FF', and Lc and the command data field } \\
\text { are absent, the command shall reset the security } \\
\text { status of the key reference in P2. The security } \\
\text { status of the key reference specified in P2 shall } \\
\text { be set to FALSE and the retry counter associated } \\
\text { with the key reference shall remain unchanged. }\end{array}$ & $\begin{array}{l}\text { - [SP800-73], Part 2, } \\
\text { Section 3.2.1 - } \\
\text { VERIFY Card } \\
\text { Command }\end{array}$ \\
\hline DTR-06.03.05.06 & $\begin{array}{l}\text { If the token command succeeds, then the security } \\
\text { status of the key reference (i.e., ' } 80 \text { ') shall be set } \\
\text { to TRUE and the retry counter associated with } \\
\text { the key reference shall be set to the reset retry } \\
\text { value associated with the key reference. }\end{array}$ & $\begin{array}{l}\text { - } \text { [SP800-73], Part 2, } \\
\text { Section 3.2.1 - } \\
\text { VERIFY Card } \\
\text { Command }\end{array}$ \\
\hline
\end{tabular}

\subsubsection{CHANGE REFERENCE DATA Command}

\begin{tabular}{|c|c|c|}
\hline DTR No: & DTR Description & Spec. Reference \\
\hline DTR-06.03.06.01 & $\begin{array}{l}\text { Only reference data associated with key } \\
\text { references '80' and '81' specific to the Derived } \\
\text { PIV Application (i.e., local key references) may } \\
\text { be changed by the Derived PIV Application } \\
\text { CHANGE REFERENCE DATA command. The } \\
\text { PIV Card Application may allow the reference } \\
\text { data associated with other key references to be } \\
\text { changed by the PIV Card Application CHANGE } \\
\text { REFERENCE DATA, if PIV Card Application } \\
\text { will only perform the command with other key } \\
\text { references if the requirements specified in } \\
\text { Section } 2.9 .2 \text { of FIPS 201-2 are satisfied. }\end{array}$ & $\begin{array}{l}\text { - } \text { [SP800-157], } \\
\text { Appendix B.2 - } \\
\text { Derived PIV } \\
\text { Application Token } \\
\text { Command Interface } \\
\text { - } \text { [SP800-73], Part 2, } \\
\text { Section 3.2.2 - } \\
\text { CHANGE } \\
\text { REFERENCE } \\
\text { DATA Card } \\
\text { Command }\end{array}$ \\
\hline DTR-06.03.06.02 & $\begin{array}{l}\text { If any key reference value is specified that is not } \\
\text { supported by the card, the Derived PIV } \\
\text { Application shall return the status word ' } 6 \mathrm{~A} \mathrm{88'.}\end{array}$ & $\begin{array}{l}\text { - } \quad \text { [SP800-157], } \\
\text { Appendix B.2 - } \\
\text { Derived PIV } \\
\text { Application Token } \\
\text { Command Interface } \\
\text { - } \quad \text { [SP800-73], Part 2, } \\
\text { Section 3.2.2 - } \\
\text { CHANGE } \\
\text { REFERENCE } \\
\text { DATA Card } \\
\text { Command }\end{array}$ \\
\hline
\end{tabular}




\begin{tabular}{|c|c|c|}
\hline DTR No: & DTR Description & Spec. Reference \\
\hline DTR-06.03.06.03 & $\begin{array}{l}\text { Key reference '80' reference data shall be } \\
\text { changed by the Derived PIV Application } \\
\text { CHANGE REFERENCE DATA command. The } \\
\text { ability to change reference data associated with } \\
\text { key references ' } 81 \text { ' using the Derived PIV } \\
\text { Application CHANGE REFERENCE DATA } \\
\text { command is optional. }\end{array}$ & $\begin{array}{ll}\text { - } & \text { [SP800-157], } \\
\text { Appendix B.2 - } \\
\text { Derived PIV } \\
\text { Application Token } \\
\text { Command Interface } \\
\text { - } \quad \text { [SP800-73], Part 2, } \\
\text { Section 3.2.2 - } \\
\text { CHANGE } \\
\text { REFERENCE } \\
\text { DATA Card } \\
\text { Command }\end{array}$ \\
\hline DTR-06.03.06.04 & $\begin{array}{l}\text { If the current value of the retry counter } \\
\text { associated with the key reference is zero, then } \\
\text { the reference data associated with the key } \\
\text { reference (i.e., ' } 80 \text { ' or ' } 81 \text { ') shall not be changed } \\
\text { and the Derived PIV Application shall return the } \\
\text { status word '69 83'. }\end{array}$ & $\begin{array}{l}\text { [SP800-157], } \\
\text { Appendix B.2 - } \\
\text { Derived PIV } \\
\text { Application Token } \\
\text { Command Interface } \\
\text { - } \text { [SP800-73], Part 2, } \\
\text { Section 3.2.2 - } \\
\text { CHANGE } \\
\text { REFERENCE } \\
\text { DATA Card } \\
\text { Command }\end{array}$ \\
\hline DTR-06.03.06.05 & $\begin{array}{l}\text { If the authentication data in the command data } \\
\text { field does not match the current value of the } \\
\text { reference data or if either the authentication data } \\
\text { or the new reference data in the command data } \\
\text { field of the command does not satisfy the criteria } \\
\text { in Appendix B.2.1 of [SP800-157] (for the } \\
\text { Derived PIV Application Password) or the } \\
\text { criteria in Section } 2.4 .3 \text { of [SP800-73], Part } 2 \\
\text { (for the PUK), the Derived PIV Application shall } \\
\text { not change the reference data associated with the } \\
\text { key reference and shall return either status word } \\
\text { '6A } 80 \text { ' or '63 CX'. }\end{array}$ & $\begin{array}{l}\text { - } \text { [SP800-157], } \\
\text { Appendix B.2 - } \\
\text { Derived PIV } \\
\text { Application Token } \\
\text { Command Interface } \\
\text { - } \quad \text { [SP800-73], Part 2, } \\
\text { Section 3.2.2 - } \\
\text { CHANGE } \\
\text { REFERENCE } \\
\text { DATA Card } \\
\text { Command }\end{array}$ \\
\hline DTR-06.03.06.06 & $\begin{array}{l}\text { If the authentication data in the command data } \\
\text { field satisfies the criteria in Appendix B.2.1 of } \\
\text { [SP800-157] (for the Derived PIV Application } \\
\text { Password) or the criteria in Section 2.4.3 of } \\
\text { [SP800-73], Part } 2 \text { (for the PUK), and matches } \\
\text { the current value of the reference data, but the } \\
\text { new reference data in the command data field of } \\
\text { the command does not satisfy the criteria in } \\
\text { Appendix B.2.1 of [SP800-157] (for the Derived }\end{array}$ & $\begin{array}{l}\text { - } \text { [SP800-157], } \\
\text { Appendix B.2 - } \\
\text { Derived PIV } \\
\text { Application Token } \\
\text { Command Interface } \\
\text { - } \quad \text { [SP800-73], Part 2, } \\
\text { Section 3.2.2 - } \\
\text { CHANGE } \\
\text { REFERENCE }\end{array}$ \\
\hline
\end{tabular}




\begin{tabular}{|c|c|c|}
\hline DTR No: & DTR Description & Spec. Reference \\
\hline & $\begin{array}{l}\text { PIV Application Password) or the criteria in } \\
\text { Section } 2.4 .3 \text { of [SP800-73], Part } 2 \text { (for the } \\
\text { PUK), the Derived PIV Application shall return } \\
\text { status word '6A 80'. }\end{array}$ & $\begin{array}{l}\text { DATA Card } \\
\text { Command }\end{array}$ \\
\hline DTR-06.03.06.07 & $\begin{array}{l}\text { If the authentication data in the command data } \\
\text { field does not match the current value of the } \\
\text { reference data, but both the authentication data } \\
\text { and the new reference data in the command data } \\
\text { field of the command satisfy the criteria in } \\
\text { Appendix B.2.1 of [SP800-157] (for the Derived } \\
\text { PIV Application Password) or the criteria in } \\
\text { Section } 2.4 .3 \text { of [SP800-73], Part } 2 \text { (for the } \\
\text { PUK), the Derived PIV Application shall return } \\
\text { status word '63 CX'. }\end{array}$ & $\begin{array}{ll}\text { - } & \text { [SP800-157], } \\
\text { Appendix B.2 - } \\
\text { Derived PIV } \\
\text { Application Token } \\
\text { Command Interface } \\
\text { - } \quad \text { [SP800-73], Part 2, } \\
\text { Section 3.2.2 - } \\
\text { CHANGE } \\
\text { REFERENCE } \\
\text { DATA Card } \\
\text { Command }\end{array}$ \\
\hline DTR-06.03.06.08 & $\begin{array}{l}\text { If status word '6A 80' is returned, the security } \\
\text { status and retry counter associated with the key } \\
\text { reference shall remain unchanged. }\end{array}$ & $\begin{array}{l}\text { - } \quad \text { [SP800-157], } \\
\text { Appendix B.2 - } \\
\text { Derived PIV } \\
\text { Application Token } \\
\text { Command Interface } \\
\text { - } \quad \text { [SP800-73], Part 2, } \\
\text { Section 3.2.2 - } \\
\text { CHANGE } \\
\text { REFERENCE } \\
\text { DATA Card } \\
\text { Command }\end{array}$ \\
\hline DTR-06.03.06.09 & $\begin{array}{l}\text { If status word ' } 63 \mathrm{CX} \text { ' is returned, the security } \\
\text { status of the key reference shall be set to FALSE } \\
\text { and the retry counter associated with the key } \\
\text { reference shall be decremented by one. }\end{array}$ & $\begin{array}{l}\text { - } \quad \text { [SP800-157], } \\
\text { Appendix B.2 - } \\
\text { Derived PIV } \\
\text { Application Token } \\
\text { Command Interface } \\
\text { - } \quad \text { SSP800-73], Part 2, } \\
\text { Section 3.2.2 - } \\
\text { CHANGE } \\
\text { REFERENCE } \\
\text { DATA Card } \\
\text { Command }\end{array}$ \\
\hline
\end{tabular}




\subsubsection{RESET RETRY COUNTER Command}

\begin{tabular}{|c|c|c|}
\hline DTR No: & DTR Description & Spec. Reference \\
\hline DTR-06.03.07.01 & $\begin{array}{l}\text { The only key reference allowed in the P2 } \\
\text { parameter of the RESET RETRY COUNTER } \\
\text { command is the Derived PIV Application } \\
\text { Password (i.e., key reference ' } 80 \text { '). The PIV } \\
\text { Card Application may allow the reference data } \\
\text { associated with other key references to be } \\
\text { changed by the PIV Card Application RESET } \\
\text { RETRY COUNTER, if PIV Card Application } \\
\text { will only perform the command with other key } \\
\text { references if the requirements specified in } \\
\text { Section } 2.9 .2 \text { of FIPS 201-2 are satisfied. If a } \\
\text { key reference is specified in P2 that is not } \\
\text { supported by the card, the Derived PIV } \\
\text { Application shall return the status word '6A } \\
88 \text { '. }\end{array}$ & $\begin{array}{ll} & \text { [SP800-157], } \\
\text { Appendix B.2 - } \\
\text { Derived PIV } \\
\text { Application Token } \\
\text { Command Interface } \\
\text { - } \quad \text { [SP800-73], Part 2, } \\
\text { Section 3.2.3 - } \\
\text { RESET RETRY } \\
\text { COUNTER Card } \\
\text { Command }\end{array}$ \\
\hline DTR-06.03.07.02 & $\begin{array}{l}\text { If the current value of the PUK's retry counter } \\
\text { is zero then the password's retry counter shall } \\
\text { not be reset and the Derived PIV Application } \\
\text { shall return the status word '69 83'. }\end{array}$ & $\begin{array}{ll}\text { - } & \text { [SP800-157], } \\
\text { Appendix B.2 - } \\
\text { Derived PIV } \\
\text { Application Token } \\
\text { Command Interface } \\
\text { - } \quad \text { [SP800-73], Part 2, } \\
\text { Section 3.2.3 - } \\
\text { RESET RETRY } \\
\text { COUNTER Card } \\
\text { Command }\end{array}$ \\
\hline DTR-06.03.07.03 & $\begin{array}{l}\text { If the reset retry counter authentication data } \\
\text { (PUK) in the command data field of the } \\
\text { command does not match reference data } \\
\text { associated with the PUK then the Derived PIV } \\
\text { Application shall return the status word '63 } \\
\text { CX'. }\end{array}$ & $\begin{array}{ll}\text { - } & \text { [SP800-157], } \\
\text { Appendix B.2 - } \\
\text { Derived PIV } \\
\text { Application Token } \\
\text { Command Interface } \\
\text { - } \quad \text { SPP80-73], Part 2, } \\
\text { Section 3.2.3 - } \\
\text { RESET RETRY } \\
\text { COUNTER Card } \\
\text { Command }\end{array}$ \\
\hline DTR-06.03.07.04 & $\begin{array}{l}\text { If the new reference data (password) in the } \\
\text { command data field of the command does not } \\
\text { satisfy the criteria in Appendix B.2.1 of } \\
\text { [SP800-157], then the Derived PIV } \\
\text { Application shall return the status word '6A } \\
\text { 80'. }\end{array}$ & $\begin{array}{ll} & \text { [SP800-157], } \\
& \text { Appendix B.2 - } \\
\text { Derived PIV } \\
\text { Application Token } \\
\text { Command Interface } \\
\text { - } \quad \text { [SP800-73], Part 2, }\end{array}$ \\
\hline
\end{tabular}




\begin{tabular}{|c|c|c|}
\hline DTR No: & DTR Description & Spec. Reference \\
\hline & & $\begin{array}{l}\text { Section 3.2.3 - } \\
\text { RESET RETRY } \\
\text { COUNTER Card } \\
\text { Command }\end{array}$ \\
\hline DTR-06.03.07.05 & $\begin{array}{l}\text { If the reset retry counter authentication data } \\
\text { (PUK) in the command data field of the } \\
\text { command does not match reference data } \\
\text { associated with the PUK and the new reference } \\
\text { data (password) in the command data field of } \\
\text { the command does not satisfy the criteria in } \\
\text { Appendix B.2.1 of [SP800-157], then the } \\
\text { Derived PIV Application shall return either } \\
\text { status word '6A 80' or '63 CX'. }\end{array}$ & $\begin{array}{ll}\text { - } & \text { [SP800-157], } \\
\text { Appendix B.2 - } \\
\text { Derived PIV } \\
\text { Application Token } \\
\text { Command Interface } \\
\text { - } \quad \text { [SP800-73], Part 2, } \\
\text { Section 3.2.3 - } \\
\text { RESET RETRY } \\
\text { COUNTER Card } \\
\text { Command }\end{array}$ \\
\hline DTR-06.03.07.06 & $\begin{array}{l}\text { If the Derived PIV Application returns status } \\
\text { word '6A } 80 \text { ' then the retry counter associated } \\
\text { with the password shall not be reset, the } \\
\text { security status of the password's key reference } \\
\text { shall remain unchanged, and the PUK's retry } \\
\text { counter shall remain unchanged. }\end{array}$ & $\begin{array}{ll}\text { - } & \text { [SP800-157], } \\
\text { Appendix B.2 - } \\
\text { Derived PIV } \\
\text { Application Token } \\
\text { Command Interface } \\
\text { - } \quad \text { [SP800-73], Part 2, } \\
\text { Section 3.2.3 - } \\
\text { RESET RETRY } \\
\text { COUNTER Card } \\
\text { Command }\end{array}$ \\
\hline DTR-06.03.07.07 & $\begin{array}{l}\text { If the Derived PIV Application returns status } \\
\text { word ' } 63 \text { CX', then the retry counter associated } \\
\text { with the password shall not be reset, the } \\
\text { security status of the password's key reference } \\
\text { shall be set to FALSE, and the PUK's retry } \\
\text { counter shall be decremented by one. }\end{array}$ & $\begin{array}{ll}\text { - } & \text { [SP800-157], } \\
\text { Appendix B.2 - } \\
\text { Derived PIV } \\
\text { Application Token } \\
\text { Command Interface } \\
\text { - } \quad \text { [SP800-73], Part 2, } \\
\text { Section 3.2.3 - } \\
\text { RESET RETRY } \\
\text { COUNTER Card } \\
\text { Command }\end{array}$ \\
\hline DTR-06.03.07.08 & $\begin{array}{l}\text { If the token command succeeds, then the } \\
\text { password's retry counter shall be set to its reset } \\
\text { retry value. Optionally, the PUK's retry } \\
\text { counter may be set to its initial reset retry } \\
\text { value. The security status of the password's } \\
\text { key reference shall not be changed. }\end{array}$ & $\begin{array}{ll} & \text { [SP800-157], } \\
& \text { Appendix B.2 - } \\
& \text { Derived PIV } \\
& \text { Application Token } \\
& \text { Command Interface } \\
& \text { [SP800-73], Part 2, } \\
& \text { Section 3.2.3 - }\end{array}$ \\
\hline
\end{tabular}




\begin{tabular}{|l|c|c|}
\hline DTR No: & DTR Description & Spec. Reference \\
\hline & & RESET RETRY \\
& & COUNTER Card \\
& & Command \\
\hline
\end{tabular}

\subsubsection{PUT DATA Command}

\begin{tabular}{|c|c|c|}
\hline DTR No: & DTR Description & Spec. Reference \\
\hline DTR-06.03.08.01 & $\begin{array}{l}\text { The PUT DATA command shall completely } \\
\text { replace the data content of a single data object } \\
\text { in the Derived PIV Application with new } \\
\text { content. }\end{array}$ & $\begin{array}{l}\text { - } \quad \text { SP800-157], } \\
\text { Appendix B.2 - } \\
\text { Derived PIV } \\
\text { Application Token } \\
\text { Command } \\
\text { Interface } \\
\text { - } \\
\text { [SP800-73], Part 2, } \\
\text { Section 3.2.5 - } \\
\text { PUT DATA Card } \\
\text { Command }\end{array}$ \\
\hline
\end{tabular}

\subsubsection{GENERATE ASYMMETRIC KEY PAIR Command}

\begin{tabular}{|c|c|c|}
\hline DTR No: & DTR Description & Spec. Reference \\
\hline DTR-06.03.09.01 & $\begin{array}{l}\text { The GENERATE ASYMMETRIC KEY PAIR } \\
\text { command initiates the generation and storing in } \\
\text { the token of the reference data of an } \\
\text { asymmetric key pair, i.e., a public key and a } \\
\text { private key. The public key of the generated } \\
\text { key pair is returned as the response to the } \\
\text { command. }\end{array}$ & $\begin{array}{l}\text { • [SP800-157], } \\
\text { Appendix B.2 - } \\
\text { Derived PIV } \\
\text { Application Token } \\
\text { Command Interface } \\
\text { • [SP800-73], Part 2, } \\
\text { Section 3.2.6 - } \\
\text { GENERATE } \\
\text { ASYMMETRIC KEY } \\
\text { PAIR Command }\end{array}$ \\
\hline DTR-06.03.09.02 & $\begin{array}{l}\text { If there is reference data currently associated } \\
\text { with the key reference, it is replaced in full by } \\
\text { the generated data. }\end{array}$ & $\begin{array}{l}\text { - [SP800-157], } \\
\text { Appendix B.2 - } \\
\text { Derived PIV } \\
\text { Application Token } \\
\text { Command Interface } \\
\text { - [SP800-73], Part 2, } \\
\text { Section 3.2.6 - } \\
\text { GENERATE } \\
\text { ASYMMETRIC KEY } \\
\text { PAIR Command }\end{array}$ \\
\hline
\end{tabular}




\section{Derived Test Requirements for Data Model of the Derived PIV Application}

This section lists requirements that apply to the Data Model of the Derived PIV Application. They are aimed towards issuers of tokens to ensure that Derived PIV Application data objects are formatted correctly and field values are in accordance to the specification.

\subsection{BER-TLV Conformance}

\subsubsection{General}

\begin{tabular}{|l|l|l|}
\hline DTR No: & DTR Description & Spec. Reference \\
\hline DTR-07.01.01.01 & $\begin{array}{l}\text { Before the card is issued, data objects that are } \\
\text { created but not used shall be set to zero-length } \\
\text { value. }\end{array}$ & $\begin{array}{l}\bullet \text { [SP800-73], Part 1, } \\
\text { Section 4.1.1 - Data } \\
\text { Object Content }\end{array}$ \\
\hline
\end{tabular}

\subsubsection{X.509 Certificate for Derived PIV Authentication}

\begin{tabular}{|l|l|l|}
\hline DTR No: & DTR Description & Spec. Reference \\
\hline DTR-07.01.02.01 & The X.509 Certificate for Derived PIV & $\bullet$ [SP800-157], \\
& Authentication shall include all the Tag- & Appendix B.1.2 - \\
& Length-Value (TLV) elements in Table 10 of & Derived PIV \\
& [SP800-73], Part 1 in the order listed. & Application Data \\
& & Model Elements \\
& & $\bullet$ [SP800-73], Part 1, \\
& & Appendix A - PIV \\
& & Data Model, Table 10 \\
\hline
\end{tabular}

\subsubsection{X.509 Certificate for Digital Signature}

\begin{tabular}{|l|l|l|}
\hline DTR No: & DTR Description & Spec. Reference \\
\hline DTR-07.01.03.01 & If implemented, the X.509 Certificate for & $\bullet$ [SP800-157], \\
& Digital Signature data object shall include all & Appendix B.1.2 - \\
& the TLV elements in Table 15 of [SP800-73], & Derived PIV \\
& Part 1 in the order listed. & Application Data \\
& & Model Elements \\
& & • [SP800-73], Part 1, \\
& & Appendix A - PIV \\
& & Data Model, Table 15 \\
\hline
\end{tabular}




\subsubsection{X.509 Certificate for Key Management}

\begin{tabular}{|l|l|l|}
\hline DTR No: & DTR Description & Spec. Reference \\
\hline DTR-07.01.04.01 & If implemented, the X.509 Certificate for Key & $\bullet$ [SP800-157], \\
& Management data object shall include all the & Appendix B.1.2 - \\
& TLV elements in Table 16 of [SP800-73], Part & Derived PIV \\
& 1 in the order listed. & Application Data \\
& & Model Elements \\
& & $\begin{array}{l}\text { [SP800-73], Part 1, } \\
\text { Appendix A - PIV } \\
\end{array}$ \\
& & Data Model, Table 16 \\
\hline
\end{tabular}

\subsubsection{Discovery Object}

\begin{tabular}{|c|c|c|}
\hline DTR No: & DTR Description & Spec. Reference \\
\hline DTR-07.01.05.01 & $\begin{array}{l}\text { If implemented, the Discovery Object shall } \\
\text { include all the TLV elements in Table } 18 \text { of } \\
\text { [SP800-73], Part } 1 \text { in the order listed. }\end{array}$ & $\begin{array}{l}\text { - } \text { [SP800-157], } \\
\text { Appendix B.1.2 - } \\
\text { Derived PIV } \\
\text { Application Data } \\
\text { Model Elements } \\
\text { - [SP800-73], Part 1, } \\
\text { Appendix A - PIV } \\
\text { Data Model, Table } 18\end{array}$ \\
\hline DTR-07.01.05.02 & $\begin{array}{l}\text { If the Discovery Object is implemented, the } \\
\text { first byte of the PIN Usage Policy shall be set } \\
\text { to 0x40. }\end{array}$ & $\begin{array}{l}\text { - [SP800-157], } \\
\text { Appendix B.1.2 - } \\
\text { Derived PIV } \\
\text { Application Data } \\
\text { Model Elements }\end{array}$ \\
\hline DTR-07.01.05.03 & $\begin{array}{l}\text { If the Discovery Object is implemented, the } \\
\text { second byte of the PIN Usage Policy shall be set } \\
\text { to } 0 x 00 \text {. }\end{array}$ & $\begin{array}{l}\text { - } \text { [SP800-73], Part 1, } \\
\text { Section 3.3.2- } \\
\text { Discovery Object }\end{array}$ \\
\hline
\end{tabular}

\subsubsection{Key History Object}

\begin{tabular}{|l|l|l|}
\hline DTR No: & DTR Description & Spec. Reference \\
\hline DTR-07.01.06.01 & If implemented, the Key History Object shall & $\bullet$ [SP800-157], \\
& include all the TLV elements in Table 19 of & Appendix B.1.2 - \\
& [SP800-73], Part 1 in the order listed. & Derived PIV \\
& & Application Data \\
& & Model Elements \\
& & • [SP800-73], Part 1, \\
& Appendix A - PIV \\
\hline
\end{tabular}




\begin{tabular}{|l|l|c|}
\hline DTR No: & DTR Description & Spec. Reference \\
\hline & & Data Model, Table 19 \\
\hline
\end{tabular}

\subsubsection{Retired X.509 Certificates for Key Management}

\begin{tabular}{|c|c|c|}
\hline DTR No: & DTR Description & Spec. Reference \\
\hline DTR-07.01.07.01 & $\begin{array}{l}\text { If implemented, the Retired X.509 Certificate } \\
\text { for Key Management data objects shall } \\
\text { include all the TLV elements in Tables } 20 \text { - } 39 \\
\text { of [SP800-73], Part } 1 \text { in the order listed. }\end{array}$ & $\begin{array}{l}\text { - } \text { [SP800-157], } \\
\text { Appendix B.1.2 - } \\
\text { Derived PIV } \\
\text { Application Data } \\
\text { Model Elements } \\
\text { - } \text { [SP800-73], Part 1, } \\
\text { Appendix A - PIV } \\
\text { Data Model, Table } \\
\text { 20-Table 39 }\end{array}$ \\
\hline
\end{tabular}

\subsubsection{Security Object}

\begin{tabular}{|c|c|c|}
\hline DTR No: & DTR Description & Spec. Reference \\
\hline DTR-07.01.08.01 & $\begin{array}{l}\text { If implemented, the Security Object shall } \\
\text { include all the TLV elements in Table } 12 \text { of } \\
\text { [SP800-73], Part } 1 \text { in the order listed. }\end{array}$ & $\begin{array}{l}\text { - } \text { [SP800-157], } \\
\text { Appendix B.1.2 - } \\
\text { Derived PIV } \\
\text { Application Data } \\
\text { Model Elements } \\
\text { - } \text { [SP800-73], Part 1, } \\
\text { Appendix A - PIV } \\
\text { Data Model, Table } 12\end{array}$ \\
\hline DTR-07.01.08.02 & $\begin{array}{l}\text { The Security Object shall be present in the } \\
\text { Derived PIV Application if either the } \\
\text { Discovery Object or the Key History object is } \\
\text { present, and shall be absent otherwise. }\end{array}$ & $\begin{array}{l}\text { - } \text { [SP800-157], } \\
\text { Appendix B.1.2 - } \\
\text { Derived PIV } \\
\text { Application Data } \\
\text { Model Elements }\end{array}$ \\
\hline DTR-07.01.08.03 & $\begin{array}{l}\text { All unsigned data objects (i.e., the Discovery } \\
\text { Object and the Key History object) within the } \\
\text { Derived PIV Application shall be included in } \\
\text { the Security Object. }\end{array}$ & $\begin{array}{l}\text { - } \text { [SP800-157], } \\
\text { Appendix B.1.2 - } \\
\text { Derived PIV } \\
\text { Application Data } \\
\text { Model Elements }\end{array}$ \\
\hline
\end{tabular}




\subsection{Signed Data Object Conformance}

\subsubsection{Security Object}

\begin{tabular}{|c|c|c|}
\hline DTR No: & DTR Description & Spec. Reference \\
\hline DTR-07.02.01.01 & $\begin{array}{l}\text { The message digests produced as a result of a } \\
\text { hash function on the contents of a Discovery } \\
\text { Object and/or the Key History Object, if } \\
\text { implemented, shall be identical to that data } \\
\text { object's message digest contained in the } \\
\text { Security Object. }\end{array}$ & $\begin{array}{ll} & \text { [SP800-157], } \\
& \text { Appendix B.1.2 - } \\
\text { Derived PIV } \\
\text { Application Data } \\
\text { Model Elements } \\
\text { - } \quad \text { [SP800-73], Part 1, } \\
\text { Section 3.1.7 - } \\
\text { Security Object }\end{array}$ \\
\hline DTR-07.02.01.02 & $\begin{array}{l}\text { The Security Object shall contain an } \\
\text { asymmetric digital signature as specified in } \\
\text { Request for Comments (RFC) 5652, } \\
\text { Cryptographic Message Syntax [RFC5652]. }\end{array}$ & $\begin{array}{l}\text { [SP800-73], Part 1, } \\
\text { Section 3.1.7- } \\
\text { Security Object }\end{array}$ \\
\hline DTR-07.02.01.03 & $\begin{array}{l}\text { The digital signature is implemented as a } \\
\text { SignedData Type. }\end{array}$ & $\begin{array}{l}\text { - } \quad \text { SP800-73], Part 1, } \\
\text { Section 3.1.7- } \\
\text { Security Object }\end{array}$ \\
\hline DTR-07.02.01.04 & $\begin{array}{l}\text { The value of the version field of the } \\
\text { SignedData content type shall be v3. }\end{array}$ & $\begin{array}{l}\text { - } \quad \text { SP800-73], Part 1, } \\
\text { Section 3.1.7- } \\
\text { Security Object }\end{array}$ \\
\hline DTR-07.02.01.05 & $\begin{array}{l}\text { The digestAlgorithms field of the SignedData } \\
\text { content type shall be in accordance with Table } \\
\text { 3-2 of [SP800-78]. }\end{array}$ & $\begin{array}{l}\text { - } \text { [SP800-73], Part 1, } \\
\text { Section 3.1.7 - } \\
\text { Security Object }\end{array}$ \\
\hline DTR-07.02.01.06 & $\begin{array}{l}\text { The eContentType of the encapContentInfo } \\
\text { shall be id-icao-ldsSecurityObject (OID = } \\
\text { 1.3.27.1.1.1). }\end{array}$ & $\begin{array}{l}\text { - } \text { [SP800-73], Part 1, } \\
\text { Section 3.1.7 - } \\
\text { Security Object }\end{array}$ \\
\hline DTR-07.02.01.07 & $\begin{array}{l}\text { The eContent of the encapContentsInfo field } \\
\text { shall contain the encoded contents of the } \\
\text { ldsSecurity object. }\end{array}$ & $\begin{array}{l}\text { - } \text { [SP800-73], Part 1, } \\
\text { Section 3.1.7- } \\
\text { Security Object }\end{array}$ \\
\hline DTR-07.02.01.08 & $\begin{array}{l}\text { The signature field of the Security Object, tag } \\
\text { 0xBB, shall include the Derived PIV } \\
\text { Credential Issuer's (content signing) } \\
\text { certificate. }\end{array}$ & $\begin{array}{l}\text { [SP800-157], } \\
\text { Appendix B.1.2 - } \\
\text { Derived PIV } \\
\text { Application Data } \\
\text { Model Elements }\end{array}$ \\
\hline DTR-07.02.01.09 & $\begin{array}{l}\text { The digestAlgorithm field specified in the } \\
\text { SignerInfo field is in accordance with Table 3- } \\
2 \text { of [SP800-78]. }\end{array}$ & $\begin{array}{l}\text { - } \quad \text { SP800-73], Part 1, } \\
\text { Section 3.1.7 - } \\
\text { Security Object }\end{array}$ \\
\hline DTR-07.02.01.10 & $\begin{array}{l}\text { The signatureAlgorithm field in the SignerInfo } \\
\text { field is specified as follows: for RSA with }\end{array}$ & $\begin{array}{l}\text { - } \quad \text { [SP800-73], Part 1, } \\
\text { Section 3.1.7 - } \\
\end{array}$ \\
\hline
\end{tabular}




\begin{tabular}{|l|l|c|}
\hline DTR No: & DTR Description & Spec. Reference \\
\hline & $\begin{array}{l}\text { PKCS \#1 v1.5 padding, the } \\
\text { signatureAlgorithm field shall specify the } \\
\text { rsaEncryption OID (as per Section 3.2 of } \\
\text { [RFC3370]), and for ECDSA and RSA with } \\
\text { PSS padding, the signatureAlgorithm shall be } \\
\text { in accordance with Table 3-3 of [SP800-78]. }\end{array}$ & \\
\hline DTR-07.02.01.11 & $\begin{array}{l}\text { The SignedData content type shall include the } \\
\text { digital signature. }\end{array}$ & $\begin{array}{l}\text { Security Objo0-73], Part 1, } \\
\text { [Section 3.1.7 - } \\
\text { Security Object }\end{array}$ \\
\hline
\end{tabular}

\subsection{PKI Conformance}

\subsubsection{X.509 Certificate for Derived PIV Authentication}

\begin{tabular}{|c|c|c|}
\hline DTR No: & DTR Description & Spec. Reference \\
\hline DTR-07.03.01.01 & $\begin{array}{l}\text { The signature field in the certificate shall } \\
\text { specify an algorithm from Table 3-3 of } \\
\text { [SP800-78] in the AlgorithmIdentifier field. }\end{array}$ & $\begin{array}{l}\text { - [SP800-78], Section } \\
\text { 3.2.1 - Specification } \\
\text { of Digital } \\
\text { Signatures on } \\
\text { Authentication } \\
\text { Information }\end{array}$ \\
\hline DTR-07.03.01.02 & $\begin{array}{l}\text { If RSA with PSS padding is used, the } \\
\text { parameters field of the AlgorithmIdentifier } \\
\text { type shall assert SHA-256 (OID = } \\
\text { 2.16.840.1.101.3.4.2.1). For RSA with PKCS } \\
\# 1 \text { v1.5 padding, the parameters field is } \\
\text { populated with NULL. For ECDSA, the } \\
\text { parameters field is absent. }\end{array}$ & $\begin{array}{l}\text { - } \text { [PROF], Worksheet } \\
\text { titled - Derived PIV } \\
\text { Authentication } \\
\text { Certificate Profile }\end{array}$ \\
\hline DTR-07.03.01.03 & $\begin{array}{l}\text { The subjectPublicKeyInfo field shall assert an } \\
\text { algorithm in the AlgorithmIdentifier in } \\
\text { accordance with Table 3-4 of [SP800-78]. }\end{array}$ & $\begin{array}{l}\text { - } \text { [SP800-78], Section } \\
\text { 3.2.2 - Specification } \\
\text { of Public Keys In } \\
\text { X.509 Certificates }\end{array}$ \\
\hline DTR-07.03.01.04 & $\begin{array}{l}\text { If the public key algorithm is elliptic curve, } \\
\text { then the parameters field contains the } \\
\text { namedCurve choice populated with the OID } \\
\text { for Curve P-256 (1.2.840.10045.3.1.7). }\end{array}$ & $\begin{array}{l}\text { [PROF], Worksheet } \\
\text { titled - Derived PIV } \\
\text { Authentication } \\
\text { Certificate Profile }\end{array}$ \\
\hline DTR-07.03.01.05 & $\begin{array}{l}\text { The keyUsage extension shall assert only the } \\
\text { digitalSignature bit. No other bits shall be } \\
\text { asserted. }\end{array}$ & $\begin{array}{l}\text { - } \text { [PROF], Worksheet } \\
\text { titled - Derived PIV } \\
\text { Authentication } \\
\text { Certificate Profile }\end{array}$ \\
\hline DTR-07.03.01.06 & $\begin{array}{l}\text { The policyIdentifier field in the } \\
\text { certificatePolicies must assert id-fpki- }\end{array}$ & $\begin{array}{l}\text { - } \text { [PROF], Worksheet } \\
\text { titled - Derived PIV }\end{array}$ \\
\hline
\end{tabular}




\begin{tabular}{|c|c|c|}
\hline DTR No: & DTR Description & Spec. Reference \\
\hline & $\begin{array}{l}\text { common-derived-pivAuth_(OID = } \\
\text { 2.16.840.1.101.3.2.1.3.40) or id-fpki-common- } \\
\text { derived-pivAuth-hardware (OID = } \\
2.16 .840 .1 \cdot 101.3 .2 .1 .3 .41)\end{array}$ & $\begin{array}{l}\text { Authentication } \\
\text { Certificate Profile }\end{array}$ \\
\hline DTR-07.03.01.07 & $\begin{array}{l}\text { The subjectAltName extension shall include a } \\
\text { UUID encoded as a URN, as specified in } \\
\text { Section } 3 \text { of [RFC4122], A Universally Unique } \\
\text { IDentifier (UUID) URN Namespace. }\end{array}$ & $\begin{array}{l}\text { - }[\text { PROF], Worksheet } \\
\text { titled - Derived PIV } \\
\text { Authentication } \\
\text { Certificate Profile }\end{array}$ \\
\hline DTR-07.03.01.08 & $\begin{array}{l}\text { The piv-interim extension (OID = } \\
2.16 .840 .1 .101 .3 .6 .9 .1) \text { shall be present and } \\
\text { contain an interim_indicator field, which is } \\
\text { populated with a Boolean value. This } \\
\text { extension is not critical. }\end{array}$ & $\begin{array}{l}\text { - }[\text { PROF], Worksheet } \\
\text { titled - Derived PIV } \\
\text { Authentication } \\
\text { Certificate Profile }\end{array}$ \\
\hline DTR-07.03.01.09 & $\begin{array}{l}\text { The authorityInfoAccess field shall contain an } \\
\text { id-ad-ocsp accessMethod. The access location } \\
\text { uses the Uniform Resource Identifier (URI) } \\
\text { name form to specify the location of a } \\
\text { Hypertext Transfer Protocol (HTTP) } \\
\text { accessible Online Certificate Status Protocol } \\
\text { (OCSP) server distributing status information } \\
\text { for this certificate. }\end{array}$ & $\begin{array}{l}\text { - }[\text { PROF], Worksheet } \\
\text { titled - Derived PIV } \\
\text { Authentication } \\
\text { Certificate Profile }\end{array}$ \\
\hline DTR-07.03.01.10 & $\begin{array}{l}\text { The cRLDistributionPoints extension is } \\
\text { required and must contain an HTTP URI. The } \\
\text { URI must point to a file that has an extension } \\
\text { of ".crl" that contains the DER encoded CRL } \\
\text { that provides status information about the } \\
\text { certificate. (see [RFC2585], Internet X.509 } \\
\text { Public Key Infrastructure Operational } \\
\text { Protocols: FTP and HTTP) }\end{array}$ & $\begin{array}{l}\text { - } \text { [PROF], Worksheet } \\
\text { titled - Derived PIV } \\
\text { Authentication } \\
\text { Certificate Profile }\end{array}$ \\
\hline DTR-07.03.01.11 & $\begin{array}{l}\text { The authorityInfoAccess field shall contain an } \\
\text { id-ad-caIssuers (1.3.6.1.5.5.7.48.2) } \\
\text { accessMethod. The access location shall } \\
\text { specify the location to an HTTP accessible } \\
\text { Web server where certificates issued to the } \\
\text { issuer of this certificate may be found. The } \\
\text { URI must point to a file that has an extension } \\
\text { of ".p7c" containing a certs-only CMS } \\
\text { message (see [RFC5751], } \\
\text { Secure/Multipurpose Internet Mail Extensions } \\
\text { (S/MIME) Version 3.2 Message Specification). }\end{array}$ & $\begin{array}{l}\text { - }[\text { PROF], Worksheet } \\
\text { titled - Derived PIV } \\
\text { Authentication } \\
\text { Certificate Profile }\end{array}$ \\
\hline DTR-07.03.01.12 & $\begin{array}{l}\text { The size of the public key for the Derived PIV } \\
\text { Authentication certificate shall be in } \\
\text { accordance with Table } 3-1 \text { of [SP800-78]. }\end{array}$ & $\begin{array}{l}\text { - }[\text { SP800-78], Section } \\
3.1 \text { - PIV } \\
\text { Cryptographic Keys }\end{array}$ \\
\hline
\end{tabular}




\begin{tabular}{|l|l|l|}
\hline DTR No: & DTR Description & Spec. Reference \\
\hline DTR-07.03.01.13 & $\begin{array}{l}\text { The public key present in the Derived PIV } \\
\text { Authentication certificate shall correspond to } \\
\text { the Derived PIV Authentication private key. }\end{array}$ & $\begin{array}{l}\bullet \text { [SP800-157], } \\
\text { Appendix B.1.2 - } \\
\text { Derived PIV } \\
\text { Application Data } \\
\text { Model Elements }\end{array}$ \\
\hline DTR-07.03.01.14 & $\begin{array}{l}\text { If the public key algorithm is RSA, the } \\
\text { exponent shall be equal to 65 537. }\end{array}$ & $\begin{array}{l}\text { [SP800-78], Section } \\
\text { 3.1, PIV } \\
\text { Cryptographic Keys }\end{array}$ \\
\hline
\end{tabular}

\subsubsection{X.509 Certificate for Digital Signature}

[SP800-157] does not specify any requirements on the digital signature key and certificate. The requirements listed herein follow those specified in [FIPS201] for digital signature certificates that are not issued by legacy PKIs, ${ }^{8}$ and thus do not actually apply to the X.509 Certificate for Digital Signature stored within a Derived PIV Application, with the exception that certificates that assert the id-fpki-common-policy, id-fpki-common-hardware, or id-fpki-common-High certificate policy OID are required by the corresponding certificate policy to conform to [PROF].

\begin{tabular}{|l|l|l|}
\hline DTR No: & DTR Description & Spec. Reference \\
\hline DTR-07.03.02.01 & $\begin{array}{l}\text { The signature field in the certificate shall } \\
\text { specify an algorithm from Table 3-3 of [SP800- } \\
\text { 78] in the AlgorithmIdentifier. }\end{array}$ & $\begin{array}{l}\text { [SP800-78], Section } \\
\text { 3.2.1 - Specification } \\
\text { of Digital Signatures } \\
\text { on Authentication } \\
\text { Information }\end{array}$ \\
\hline DTR-07.03.02.02 & $\begin{array}{l}\text { If RSA with PSS padding is used, the } \\
\text { parameters field of the AlgorithmIdentifier type } \\
\text { shall assert SHA-256 (OID = } \\
\text { 2.16.840.1.101.3.4.2.1). For RSA with PKCS } \\
\text { \#1 v1.5 padding, the parameters field is } \\
\text { populated with NULL. For ECDSA, the } \\
\text { parameters field is absent. }\end{array}$ & $\begin{array}{l}\text { [PROF], Worksheet } \\
\text { titled - End Entity } \\
\text { Pignature Certificate } \\
\text { Profile }\end{array}$ \\
& $\begin{array}{l}\text { The subjectPublicKeyInfo field shall assert an } \\
\text { algorithm in the AlgorithmIdentifier in } \\
\text { accordance with Table 3-4 of [SP800-78]. }\end{array}$ & $\begin{array}{l}\text { [SP800-78], Section } \\
\text { 3.2.2 - Specification } \\
\text { of Public Keys In } \\
\text { X.509 Certificates }\end{array}$ \\
\hline DTR-07.03.02.03 & $\begin{array}{l}\text { [PROF], Worksheet } \\
\text { titled - End Entity } \\
\text { Signature Certificate } \\
\text { Profile }\end{array}$ \\
\hline DTR-07.03.02.04 & $\begin{array}{l}\text { If the public key algorithm is elliptic curve, } \\
\text { then the parameters field contains the }\end{array}$ \\
& namedCurve choice populated an appropriate \\
OID from [SP800-78]. & \\
\hline
\end{tabular}

\footnotetext{
${ }^{8}$ Legacy PKIs are the PKIs of departments and agencies that have cross-certified with the Federal Bridge CA (FBCA) at the Medium Hardware or High Assurance Level.
} 


\begin{tabular}{|c|c|c|}
\hline DTR No: & DTR Description & Spec. Reference \\
\hline DTR-07.03.02.05 & $\begin{array}{l}\text { The keyUsage extension shall assert both the } \\
\text { digitalSignature and nonRepudiation bits. No } \\
\text { other bits shall be asserted. }\end{array}$ & $\begin{array}{l}\text { - }[\text { PROF], Worksheet } \\
\text { titled - End Entity } \\
\text { Signature Certificate } \\
\text { Profile }\end{array}$ \\
\hline DTR-07.03.02.06 & $\begin{array}{l}\text { The policyIdentifier field in the } \\
\text { certificatePolicies must assert one of the } \\
\text { following: id-fpki-common-policy (OID = } \\
\text { 2.16.840.1.101.3.2.1.3.6), id-fpki-common- } \\
\text { hardware (OID = 2.16.840.1.101.3.2.1.3.7) or } \\
\text { id-fpki-common-High (OID = } \\
\text { 2.16.840.1.101.3.2.1.3.16). }\end{array}$ & $\begin{array}{l}\text { [PROF], Worksheet } \\
\text { titled - End Entity } \\
\text { Signature Certificate } \\
\text { Profile }\end{array}$ \\
\hline DTR-07.03.02.07 & $\begin{array}{l}\text { The authorityInfoAccess field shall contain an } \\
\text { id-ad-caIssuers (1.3.6.1.5.5.7.48.2) } \\
\text { accessMethod. The access location shall to } \\
\text { specify the location of an LDAP accessible } \\
\text { directory server or HTTP accessible Web server } \\
\text { where certificates issued to the issuer of this } \\
\text { certificate may be found. } \\
\text { If LDAP is used, the URI must include the DN } \\
\text { of the entry containing the relevant certificates } \\
\text { and specify the directory attribute in which the } \\
\text { certificates are located. If the directory in which } \\
\text { the certificates are stored expects the "binary" } \\
\text { option to be specified, then the attribute type } \\
\text { must be followed by ";binary" in the URI. } \\
\text { If HTTP is used, the URI must point to a file } \\
\text { that has an extension of ".p7c" containing a } \\
\text { certs-only CMS message (see RFC 5751, } \\
\text { Secure/Multipurpose Internet Mail Extensions } \\
\text { (S/MIME) Version } 3.2 \text { Message Specification } \\
\text { [RFC5751]). }\end{array}$ & $\begin{array}{l}\text { - }[\text { PROF], Worksheet } \\
\text { titled - End Entity } \\
\text { Signature Certificate } \\
\text { Profile }\end{array}$ \\
\hline DTR-07.03.02.08 & $\begin{array}{l}\text { The cRLDistributionPoints extension is } \\
\text { required and must contain at least one URI, } \\
\text { either LDAP or HTTP. } \\
\text { If LDAP is used, the URI must include the DN } \\
\text { of the entry containing the CRL and specify the } \\
\text { directory attribute in which the CRL is located } \\
\text { (certificateRevocationList). } \\
\text { If HTTP is used, the URI must point to a file } \\
\text { that has an extension of ".crl" that contains the } \\
\text { DER encoded CRL. (see [RFC2585], Internet } \\
\text { X.509 Public Key Infrastructure Operational } \\
\text { Protocols: FTP and HTTP) }\end{array}$ & $\begin{array}{l}\text { [PROF], Worksheet } \\
\text { titled - End Entity } \\
\text { Signature Certificate } \\
\text { Profile }\end{array}$ \\
\hline
\end{tabular}




\begin{tabular}{|c|c|c|}
\hline DTR No: & DTR Description & Spec. Reference \\
\hline DTR-07.03.02.09 & $\begin{array}{l}\text { The size of the public key for the digital } \\
\text { signature certificate shall be in accordance with } \\
\text { Table 3-1 of [SP800-78]. }\end{array}$ & $\begin{array}{l}\text { - }[\text { SP800-78], Section } \\
3.1 \text { - PIV } \\
\text { Cryptographic Keys }\end{array}$ \\
\hline DTR-07.03.02.10 & $\begin{array}{l}\text { The public key present in the digital signature } \\
\text { certificate shall correspond to the digital } \\
\text { signature private key. }\end{array}$ & $\begin{array}{l}\text { [SP800-157], } \\
\text { Appendix B.1.2 - } \\
\text { Derived PIV } \\
\text { Application Data } \\
\text { Model Elements }\end{array}$ \\
\hline DTR-07.03.02.11 & $\begin{array}{l}\text { If the public key algorithm is RSA, the } \\
\text { exponent shall be equal to } 65537 \text {. }\end{array}$ & $\begin{array}{l}\text { [SP800-78], Section } \\
\text { 3.1, PIV } \\
\text { Cryptographic Keys }\end{array}$ \\
\hline
\end{tabular}

\subsubsection{X.509 Certificate for Key Management}

[SP800-157] does not specify requirements on the key management key and certificate. The requirements listed herein follow those from [FIPS201] for key management certificates that are not issued by legacy PKIs, and thus do not actually apply to the X.509 Certificate for Key Management stored within a Derived PIV Application, with the exception that certificates that assert the id-fpki-common-policy, id-fpki-common-hardware, or id-fpki-common-High certificate policy OID are required by the corresponding certificate policy to conform to [PROF].

\begin{tabular}{|c|c|c|}
\hline DTR No: & DTR Description & Spec. Reference \\
\hline DTR-07.03.03.01 & $\begin{array}{l}\text { The signature field in the certificate shall } \\
\text { specify an algorithm from Table } 3-3 \text { of } \\
\text { [SP800-78] in the AlgorithmIdentifier. }\end{array}$ & $\begin{array}{l}\text { - [SP800-78], Section } \\
\text { 3.2.1 - Specification } \\
\text { of Digital } \\
\text { Signatures on } \\
\text { Authentication } \\
\text { Information }\end{array}$ \\
\hline DTR-07.03.03.02 & $\begin{array}{l}\text { If RSA with PSS padding is used, the } \\
\text { parameters field of the AlgorithmIdentifier } \\
\text { type shall assert Secure Hash Algorithm } \\
\text { (SHA) } 256 \text { (OID = 2.16.840.1.101.3.4.2.1). } \\
\text { For the other RSA algorithms, the parameters } \\
\text { field is populated with NULL. For ECDSA, } \\
\text { the parameters field is absent. }\end{array}$ & $\begin{array}{l}\text { - }[\text { PROF], Worksheet } \\
\text { titled - Key } \\
\text { Management } \\
\text { Certificate Profile }\end{array}$ \\
\hline DTR-07.03.03.03 & $\begin{array}{l}\text { The subjectPublicKeyInfo field shall assert an } \\
\text { algorithm in the AlgorithmIdentifier in } \\
\text { accordance with Table 3-4 of [SP800-78]. }\end{array}$ & $\begin{array}{l}\text { - } \text { [SP800-78], Section } \\
\text { 3.2.2 - Specification } \\
\text { of Public Keys In } \\
\text { X.509 Certificates }\end{array}$ \\
\hline DTR-07.03.03.04 & $\begin{array}{l}\text { If the public key algorithm is elliptic curve, } \\
\text { then the parameters field contains the } \\
\text { namedCurve choice populated with an } \\
\text { appropriate OID from [SP800-78]. }\end{array}$ & $\begin{array}{l}\text { - } \text { [PROF], Worksheet } \\
\text { titled - Key } \\
\text { Management } \\
\text { Certificate Profile }\end{array}$ \\
\hline
\end{tabular}




\begin{tabular}{|c|c|c|}
\hline DTR No: & DTR Description & Spec. Reference \\
\hline DTR-07.03.03.05 & $\begin{array}{l}\text { If the public key algorithm is RSA, then the } \\
\text { keyUsage extension shall only assert the } \\
\text { keyEncipherment bit. If the public key } \\
\text { algorithm is elliptic curve, then the keyUsage } \\
\text { extension shall only assert the keyAgreement } \\
\text { bit. }\end{array}$ & $\begin{array}{l}\text { - } \quad \text { PROF], Worksheet } \\
\text { titled - Key } \\
\text { Management } \\
\text { Certificate Profile }\end{array}$ \\
\hline DTR-07.03.03.06 & $\begin{array}{l}\text { The policyIdentifier field in the } \\
\text { certificatePolicies must assert one of the } \\
\text { following: id-fpki-common-policy } \square \text { (OID = } \\
\text { 2.16.840.1.101.3.2.1.3.6), id-fpki-common- } \\
\text { hardware (OID = 2.16.840.1.101.3.2.1.3.7) or } \\
\text { id-fpki-common-High (OID = } \\
2.16 .840 .1 .101 .3 .2 .1 .3 .16) \text {. }\end{array}$ & $\begin{array}{l}\text { - } \text { [PROF], Worksheet } \\
\text { titled - Key } \\
\text { Management } \\
\text { Certificate Profile }\end{array}$ \\
\hline DTR-07.03.03.07 & $\begin{array}{l}\text { The authorityInfoAccess field shall contain an } \\
\text { id-ad-caIssuers (1.3.6.1.5.5.7.48.2) } \\
\text { accessMethod. The access location shall to } \\
\text { specify the location of an LDAP accessible } \\
\text { directory server or HTTP accessible Web } \\
\text { server where certificates issued to the issuer of } \\
\text { this certificate may be found. } \\
\text { If LDAP is used, the URI must include the DN } \\
\text { of the entry containing the relevant certificates } \\
\text { and specify the directory attribute in which the } \\
\text { certificates are located. If the directory in } \\
\text { which the certificates are stored expects the } \\
\text { "binary" option to be specified, then the } \\
\text { attribute type must be followed by ";binary" in } \\
\text { the URI. } \\
\text { If HTTP is used, the URI must point to a file } \\
\text { that has an extension of ".p7c" containing a } \\
\text { certs-only CMS message (see [RFC5751], } \\
\text { Secure/Multipurpose Internet Mail Extensions } \\
\text { (S/MIME) Version 3.2 Message Specification). }\end{array}$ & $\begin{array}{l}\text { - } \quad \text { PROF], Worksheet } \\
\text { titled - Key } \\
\text { Management } \\
\text { Certificate Profile }\end{array}$ \\
\hline DTR-07.03.03.08 & $\begin{array}{l}\text { The cRLDistributionPoints extension is } \\
\text { required and must contain at least one URI, } \\
\text { either LDAP or HTTP. } \\
\text { If LDAP is used, the URI must include the DN } \\
\text { of the entry containing the CRL and specify } \\
\text { the directory attribute in which the CRL is } \\
\text { located (certificateRevocationList). } \\
\text { If HTTP is used, the URI must point to a file } \\
\text { that has an extension of ".crl" that contains the } \\
\text { DER encoded CRL. (see [RFC2585], Internet } \\
\text { X.509 Public Key Infrastructure Operational } \\
\text { Protocols: FTP and HTTP) }\end{array}$ & $\begin{array}{ll}\text { - } & \text { [PROF], Worksheet } \\
\text { titled - Key } \\
\text { Management } \\
\text { Certificate Profile }\end{array}$ \\
\hline
\end{tabular}




\begin{tabular}{|l|l|l|}
\hline DTR No: & DTR Description & Spec. Reference \\
\hline DTR-07.03.03.09 & $\begin{array}{l}\text { The size of the public key for the key } \\
\text { management certificate shall be in accordance } \\
\text { with Table 3-1 of [SP800-78]. }\end{array}$ & $\begin{array}{l}\bullet \text { [SP800-78], Section } \\
\text { 3.1 - PIV } \\
\text { Cryptographic Keys }\end{array}$ \\
\hline DTR-07.03.03.10 & $\begin{array}{l}\text { The public key present in the key management } \\
\text { certificate shall correspond to the key } \\
\text { management private key. }\end{array}$ & $\begin{array}{l}\bullet \text { [SP800-157], } \\
\text { Appendix B.1.2 - } \\
\text { Derived PIV } \\
\text { Application Data } \\
\text { Model Elements }\end{array}$ \\
& $\begin{array}{l}\text { If the public key algorithm is RSA, the } \\
\text { exponent shall be equal to 65 537. }\end{array}$ & $\begin{array}{l}\text { [SP800-78], Section } \\
\text { 3.1, PIV } \\
\text { Cryptographic Keys }\end{array}$ \\
\hline
\end{tabular}

\subsubsection{X.509 Certificate for the Derived PIV Credential Issuer (Content Signing) ${ }^{9}$}

\begin{tabular}{|c|c|c|}
\hline DTR No: & DTR Description & Spec. Reference \\
\hline DTR-07.03.04.01 & $\begin{array}{l}\text { The signature field in the certificate shall } \\
\text { specify one of the following algorithm OIDs: } \\
\text { 1.2.840.113549.1.10 (id-RSASSA-PSS), } \\
\text { 1.2.840.113549.1.11 } \\
\text { (Sha256WithRSAEncryption), } \\
\text { 1.2.840.10045.4.3.2 (edsa-with-Sha256), or } \\
\text { 1.2.840.10045.4.3.3 (edsa-with-Sha384). }\end{array}$ & $\begin{array}{l}\text { - }[\text { PROF], Worksheet } \\
\text { titled - Common } \\
\text { PIV Content Signing } \\
\text { Certificate Profile }\end{array}$ \\
\hline DTR-07.03.04.02 & $\begin{array}{l}\text { If RSA with PSS padding is used, the } \\
\text { parameters field of the AlgorithmIdentifier } \\
\text { type shall assert SHA-256 (OID = } \\
2.16 .840 .1 .101 .3 .4 .2 .1 \text { ). For RSA with PKCS } \\
\# 1 \text { v1.5 padding, the parameters field is } \\
\text { populated with NULL. For ECDSA, the } \\
\text { parameters field is absent. }\end{array}$ & $\begin{array}{l}\text { [PROF], Worksheet } \\
\text { titled - Common } \\
\text { PIV Content Signing } \\
\text { Certificate Profile }\end{array}$ \\
\hline DTR-07.03.04.03 & $\begin{array}{l}\text { The subjectPublicKeyInfo field shall assert } \\
\text { one of the following algorithm OIDs: } \\
\text { 1.2.840.113549.1.1.1 (RSA Encryption) or } \\
\text { 1.2.840.10045.2.1 (Elliptic curve key). }\end{array}$ & $\begin{array}{l}\text { [PROF], Worksheet } \\
\text { titled - Common } \\
\text { PIV Content Signing } \\
\text { Certificate Profile }\end{array}$ \\
\hline DTR-07.03.04.04 & $\begin{array}{l}\text { If the public key algorithm is elliptic curve, } \\
\text { then the parameters field contains the } \\
\text { namedCurve choice populated with one of the } \\
\text { following OIDs: } 1.2 .840 .10045 .3 .1 .7 \text { (Curve } \\
\text { P-256) or } \\
\text { 1.3.132.0.34 (Curve P-384). }\end{array}$ & $\begin{array}{l}\text { - }[\text { PROF], Worksheet } \\
\text { titled - Common } \\
\text { PIV Content Signing } \\
\text { Certificate Profile }\end{array}$ \\
\hline
\end{tabular}

\footnotetext{
${ }^{9}$ Located in the Security Object’s Cryptographic Message Syntax (CMS) signature field (tag 0xBB).
} 


\begin{tabular}{|c|c|c|}
\hline DTR No: & DTR Description & Spec. Reference \\
\hline DTR-07.03.04.05 & $\begin{array}{l}\text { The keyUsage extension shall assert the } \\
\text { digitalSignature bit. No other bits shall be } \\
\text { asserted. }\end{array}$ & $\begin{array}{l}\text { - }[\text { PROF], Worksheet } \\
\text { titled - Common } \\
\text { PIV Content Signing } \\
\text { Certificate Profile }\end{array}$ \\
\hline DTR-07.03.04.06 & $\begin{array}{l}\text { The policyIdentifier field in the } \\
\text { certificatePolicies must assert the following: } \\
\text { id-fpki-common-contentSigning } \\
(2.16 .840 .1 .101 .3 .2 .1 .3 .39) \text {. }\end{array}$ & $\begin{array}{ll} & \text { [SP800-157], } \\
\text { Appendix B.1.2 - } \\
\text { Derived PIV } \\
\text { Application Data } \\
\text { Model Elements } \\
\text { - } \quad \text { [FIPS201], Section } \\
\text { 4.2.1, Cardholder } \\
\text { Unique Identifier } \\
\text { (CHUID) }\end{array}$ \\
\hline DTR-07.03.04.07 & $\begin{array}{l}\text { The extended key usage (extKeyUsage) } \\
\text { extension shall assert the id-PIV-content- } \\
\text { signing (OID = 2.16.840.1.101.3.6.7). }\end{array}$ & $\begin{array}{ll} & \text { [SP800-157], } \\
\text { Appendix B.1.2 - } \\
\text { Derived PIV } \\
\text { Application Data } \\
\text { Model Elements } \\
\text { - } \quad \text { [FIPS201], Section } \\
\text { 4.2.1, Cardholder } \\
\text { Unique Identifier } \\
\text { (CHUID) }\end{array}$ \\
\hline DTR-07.03.04.08 & $\begin{array}{l}\text { Certificates must include an } \\
\text { authorityInfoAccess extension with at least } \\
\text { one instance of the caIssuers access method } \\
\text { (1.3.6.1.5.5.7.48.2) that specifies an HTTP } \\
\text { URI that points to a location where certificates } \\
\text { issued to the issuer of this certificate may be } \\
\text { found. } \\
\text { The HTTP URI must point to a file that has an } \\
\text { extension of ".p7c" containing a certs-only } \\
\text { CMS message (see RFC 5751, } \\
\text { Secure/Multipurpose Internet Mail Extensions } \\
\text { (S/MIME) Version 3.2 Message Specification } \\
\text { [RFC5751]). }\end{array}$ & $\begin{array}{l}\text { [PROF], Worksheet } \\
\text { titled - Common } \\
\text { PIV Content Signing } \\
\text { Certificate Profile }\end{array}$ \\
\hline DTR-07.03.04.09 & $\begin{array}{l}\text { The cRLDistributionPoints extension is } \\
\text { required and must contain at least URI, either } \\
\text { LDAP or HTTP. } \\
\text { If LDAP is used, the URI must include the DN } \\
\text { of the entry containing the CRL and specify } \\
\text { the directory attribute in which the CRL is } \\
\text { located certificateRevocationList). }\end{array}$ & $\begin{array}{l}\text { - }[\text { PROF], Worksheet } \\
\text { titled - Common } \\
\text { PIV Content Signing } \\
\text { Certificate Profile }\end{array}$ \\
\hline
\end{tabular}




\begin{tabular}{|l|l|l|}
\hline DTR No: & DTR Description & Spec. Reference \\
\hline & $\begin{array}{l}\text { If HTTP is used, the URI must point to a file } \\
\text { that has an extension of ".crl" that contains the } \\
\text { DER encoded CRL. (see [RFC2585], Internet } \\
\text { X.509 Public Key Infrastructure Operational } \\
\text { Protocols: FTP and HTTP) }\end{array}$ & \\
\hline DTR-07.03.04.10 & $\begin{array}{l}\text { The size of the subject public key in the } \\
\text { Derived PIV Credential Issuer's (content } \\
\text { signing) certificate shall conform to Table 3-2 } \\
\text { in [SP800-78]. }\end{array}$ & $\begin{array}{l}\text { [SP800-78], Section } \\
\text { of Digital Signatures } \\
\text { on Authentication } \\
\text { Information }\end{array}$ \\
& & $\begin{array}{l}\text { Infion } \\
\end{array}$ \\
\hline
\end{tabular}




\section{Test Assertions for the Derived PIV Application}

This section lists the test assertions used to determine conformity to the derived test requirements (DTR) listed in Section 6. The Implementation Under Test (IUT), in this case a Derived PIV Application submitted by a vendor, must meet the stated objective(s) of the assertion by way of a test or submission of documents/artifacts in order to be deemed conformant to the associated $\operatorname{DTR}(\mathrm{s})$.

\subsection{Transport Layer Conformance}

\subsubsection{UICC}

\subsubsection{GlobalPlatform Support for UICC Tokens}

\begin{tabular}{|l|l|}
\hline Test Assertion & TA-08.01.01.01 \\
\hline Purpose & $\begin{array}{l}\text { Confirms that for Universal Integrated Circuit Card (UICC) } \\
\text { implementations used to host a Derived PIV Application, the UICC } \\
\text { implements the GlobalPlatform Card Secure Element Configuration } \\
\text { v1.0 [GPSE]. }\end{array}$ \\
\hline DTR(s) & $\begin{array}{l}\text { DTR-06.01.01.01 } \\
\text { Vendor }\end{array}$ \\
$\begin{array}{l}\text { The vendor to provide evidence in its documentation that the UICC that } \\
\text { hosts the Derived PIV Application implements the GlobalPlatform Card } \\
\text { Secure Element Configuration v1.0 [GPSE]. }\end{array}$ \\
\hline
\end{tabular}

\subsubsection{USB}

\subsubsection{ICCD Specification Support for USB Tokens}

\begin{tabular}{|l|l|}
\hline Test Assertion & TA-08.01.02.01 \\
\hline Purpose & $\begin{array}{l}\text { Confirms that for USB Integrated Circuit(s) Card Devices (ICCD) } \\
\text { implementations used to host a Derived PIV Application, the ICCD uses } \\
\text { the Bulk-in/Bulk-Out command pipe for APDU transport and } \\
\text { implements the Universal Serial Bus Device Class - Smart Card ICCD } \\
\text { Specification for USB Integrated Circuit(s) Card Devices [ICCDSPEC]. }\end{array}$ \\
\hline DTR(s) & $\begin{array}{l}\text { DTR-06.01.02.01 } \\
\text { Vendor }\end{array}$ \\
\hline Documentation & $\begin{array}{l}\text { The vendor to provide evidence in its documentation that the ICCD that } \\
\text { hosts the Derived PIV Application implements the Universal Serial Bus } \\
\text { Device Class - Smart Card ICCD Specification for USB Integrated } \\
\text { Circuit(s) Card Devices [ICCDSPEC]. The vendor confirms that the } \\
\text { APDUs are received from the secure element using the Bulk-In } \\
\text { command pipe. }\end{array}$ \\
\hline
\end{tabular}




\subsubsection{SP 800-96 Support for USB Tokens}

\begin{tabular}{|l|l|}
\hline Test Assertion & TA-08.01.02.02 \\
\hline Purpose & $\begin{array}{l}\text { For a USB token that hosts a Derived PIV Application, confirm that the } \\
\text { token is compliant with the specifications in [SP800-96] for APDU } \\
\text { support for contact card readers. }\end{array}$ \\
\hline DTR(s) & $\bullet$ DTR-06.01.02.02 \\
\hline $\begin{array}{l}\text { Vendor } \\
\text { Documentation }\end{array}$ & $\begin{array}{l}\text { The vendor to provide evidence in its documentation that the USB token } \\
\text { is compliant with the specifications in [SP800-96] for APDU support } \\
\text { for contact card readers. }\end{array}$ \\
\hline
\end{tabular}

\subsection{Derived PIV Application Data Object Access/Storage Conformance}

\subsubsection{General}

\subsubsection{Support for Contact Interface}

\begin{tabular}{|l|l|}
\hline Test Assertion & TA-08.02.01.01 \\
\hline Purpose & $\begin{array}{l}\text { Confirms that the Derived PIV Application only supports a contact } \\
\text { interface. }\end{array}$ \\
\hline DTR(s) & $\bullet \quad$ DTR-06.02.01.01 \\
\hline $\begin{array}{l}\text { Vendor } \\
\text { Documentation }\end{array}$ & $\begin{array}{l}\text { The vendor to provide evidence in its documentation that the Derived } \\
\text { PIV Application only supports a contact interface. }\end{array}$ \\
\hline
\end{tabular}

\subsubsection{One Derived PIV Application}

\begin{tabular}{|l|l|}
\hline Test Assertion & TA-08.02.01.02 \\
\hline Purpose & $\begin{array}{l}\text { Confirms that there is only one Derived PIV Application on any } \\
\text { hardware cryptographic token. }\end{array}$ \\
\hline DTR(s) & • DTR-06.02.01.02 \\
\hline $\begin{array}{l}\text { Vendor } \\
\text { Documentation }\end{array}$ & $\begin{array}{l}\text { The vendor to provide information in its documentation validating the } \\
\text { compliance with this requirement. }\end{array}$ \\
\hline
\end{tabular}

\subsubsection{Derived PIV Application Data Objects and Representation}

\subsubsection{Derived PIV Application Data Objects}

\begin{tabular}{|l|l|}
\hline Test Assertion & TA-08.02.02.01 \\
\hline Purpose & $\begin{array}{l}\text { Confirms the data objects (along with their access conditions) are } \\
\text { implemented by the vendor of the Derived PIV Application per the } \\
\text { specification. }\end{array}$ \\
\hline
\end{tabular}




\begin{tabular}{|l|l|}
\hline DTR(s) & $\bullet \quad$ DTR-06.02.02.01 \\
\hline $\begin{array}{l}\text { Vendor } \\
\text { Documentation }\end{array}$ & $\begin{array}{l}\text { The vendor to provide documentation identifying all the data objects } \\
\text { (mandatory and optional) implemented within the Derived PIV } \\
\text { Application. }\end{array}$ \\
\hline
\end{tabular}

\subsubsection{Derived PIV Data Objects Container Capacity}

\begin{tabular}{|c|c|}
\hline Test Assertion & TA-08.02.02.02 \\
\hline Purpose & $\begin{array}{l}\text { Confirms the container capacity for all Derived PIV data objects } \\
\text { implemented on the Derived PIV Application. }\end{array}$ \\
\hline DTR(s) & $\begin{array}{ll} & \text { DTR-06.02.02.02 } \\
- & \text { DTR-06.02.02.03 } \\
\text { - } & \text { DTR-06.02.02.04 } \\
\text { - } & \text { DTR-06.02.02.05 } \\
\text { - } & \text { DTR-06.02.02.06 } \\
\text { - } & \text { DTR-06.02.02.02.07 } \\
\end{array}$ \\
\hline $\begin{array}{l}\text { Vendor } \\
\text { Documentation }\end{array}$ & $\begin{array}{l}\text { The vendor to provide in its documentation the implemented data } \\
\text { objects with their minimum container sizes on the Derived PIV } \\
\text { Application. }\end{array}$ \\
\hline
\end{tabular}

\subsubsection{Status Words}

\begin{tabular}{|c|c|}
\hline Test Assertion & TA-08.02.02.03 \\
\hline Purpose & $\begin{array}{l}\text { Confirms that all return codes are implemented by the Derived PIV } \\
\text { Application. }\end{array}$ \\
\hline DTR(s) & - DTR-06.02.02.09 \\
\hline $\begin{array}{l}\text { Vendor } \\
\text { Documentation }\end{array}$ & $\begin{array}{l}\text { The vendor to provide all the status codes returned by the Derived PIV } \\
\text { Application for the various token interface commands in its } \\
\text { documentation. The status codes are consistent with those specified in } \\
\text { Section } 5.6 \text { of [SP800-73], Part } 1 \text {. }\end{array}$ \\
\hline
\end{tabular}

\subsubsection{BER-TLV for the Derived PIV Data Objects}

\begin{tabular}{|l|l|}
\hline Test Assertion & TA-08.02.02.04 \\
\hline Purpose & $\begin{array}{l}\text { Confirms the BER-TLV tags for the data objects implemented within } \\
\text { the Derived PIV Application. }\end{array}$ \\
\hline DTR(s) & $\begin{array}{c}|c| \\
\text { DTR-06.02.02.10 }\end{array}$ \\
\hline $\begin{array}{l}\text { Vendor } \\
\text { Documentation }\end{array}$ & $\begin{array}{l}\text { The vendor to provide in its documentation the list of all the data objects } \\
\text { implemented in the Derived PIV Application with the BER-TLV tags } \\
\text { associated with each of them. }\end{array}$ \\
\hline
\end{tabular}




\subsubsection{Key Reference Values}

\begin{tabular}{|l|l|}
\hline Test Assertion & TA-08.02.02.05 \\
\hline Purpose & $\begin{array}{l}\text { Confirms that all the key references used on the Derived PIV } \\
\text { Application interfaces are in accordance with Table 6-1 of [SP800-78] } \\
\text { and Table 4a of [SP800-73], Part 1, with the mappings defined in Table } \\
\text { B-2 of [SP800-157]. }\end{array}$ \\
\hline DTR(s) & $\bullet \quad$ DTR-06.02.02.11 \\
\hline $\begin{array}{l}\text { Vendor } \\
\text { Documentation }\end{array}$ & $\begin{array}{l}\text { The vendor to provide in its documentation the key references } \\
\text { implemented by the Derived PIV Application. }\end{array}$ \\
\hline
\end{tabular}

\subsubsection{Algorithm Identifiers}

\begin{tabular}{|c|c|}
\hline Test Assertion & TA-08.02.02.06 \\
\hline Purpose & $\begin{array}{l}\text { Confirms that the required cryptographic algorithms and their identifiers } \\
\text { are implemented by the Derived PIV Application. }\end{array}$ \\
\hline DTR(s) & - $\quad$ DTR-06.02.02.12 \\
\hline $\begin{array}{l}\text { Vendor } \\
\text { Documentation }\end{array}$ & $\begin{array}{l}\text { The vendor to provide in its documentation the cryptographic } \\
\text { algorithms and their identifiers supported by the Derived PIV } \\
\text { Application. }\end{array}$ \\
\hline
\end{tabular}

\subsection{Derived PIV Application Command Interface Conformance}

\subsubsection{SELECT Command}

\subsubsection{Select using the Full and Truncated AID}

\begin{tabular}{|l|l|}
\hline Test Assertion & TA-08.03.01.01 \\
\hline Purpose & $\begin{array}{l}\text { Verifies that the Derived PIV Application executes the SELECT token } \\
\text { command for the following conditions: (i) long AID and (ii) right- } \\
\text { truncated short AID. The application property template as specified by } \\
\text { the vendor is returned. }\end{array}$ \\
\hline DTR(s) & $\begin{array}{l}\text { - } \\
\text { - }\end{array}$ \\
& $\begin{array}{l}\text { - DTR-06.02.01.03 } \\
\text { - DTR-06.03.02.02.09 }\end{array}$ \\
& $\begin{array}{l}\text { - DTR-06.03.02.03 } \\
\text { - DTR-06.03.02.04 }\end{array}$ \\
\hline Vendor & $\begin{array}{l}\text { None. } \\
\text { Documentation }\end{array}$ \\
\hline Precondition(s) & $\begin{array}{l}\text { A token with the Derived PIV Application is inserted into an } \\
\text { appropriate token reader. }\end{array}$ \\
\hline
\end{tabular}




\begin{tabular}{|c|c|}
\hline & $\begin{array}{l}\text { - Suitable drivers have been loaded between the test system and an } \\
\text { instance of the reader. }\end{array}$ \\
\hline Test Scenario & 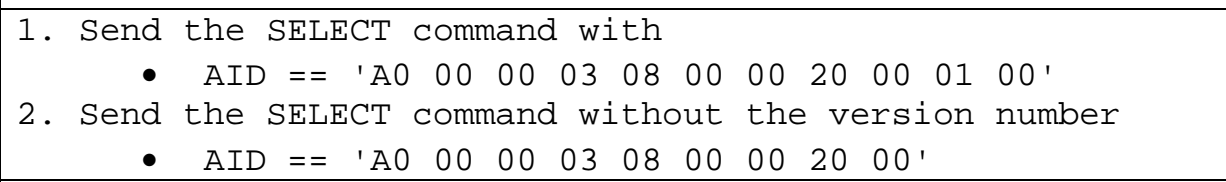 \\
\hline Expected Result(s) & $\begin{array}{l}\text { 1. From Step 1, the command returns the application property template } \\
\text { with the status word ' } 9000 \text { ' at the end. Check that the application } \\
\text { property template conforms to Table } 3 \text { of [SP800-73], Part 2, with } \\
\text { the exception that the returned AID is the AID listed in Section } \\
\text { B.1.1 of [SP800-157]. } \\
\text { 2. From Step 2, the command returns the application property template } \\
\text { with the status word ' } 9000 \text { ' at the end. Check that the application } \\
\text { property template conforms to Table } 3 \text { of [SP800-73], Part 2, with } \\
\text { the exception that the returned AID is the AID listed in Section } \\
\text { B.1.1 of [SP800-157]. }\end{array}$ \\
\hline Postcondition(s) & $\begin{array}{l}\text { The Derived PIV Application is now the currently selected application. } \\
\text { The application security status of the Derived PIV Application is } \\
\text { established. }\end{array}$ \\
\hline
\end{tabular}

\subsubsection{Default Selected Application}

\begin{tabular}{|l|l|}
\hline Test Assertion & TA-08.03.01.02 \\
\hline Purpose & $\begin{array}{l}\text { Confirms that a default selected application exists on the hardware } \\
\text { token. }\end{array}$ \\
\hline DTR(s) & $\begin{array}{l}\text { - DTR-06.02.02.09 } \\
\text { - DTR-06.03.02.02 }\end{array}$ \\
\hline $\begin{array}{l}\text { Vendor } \\
\text { Documentation }\end{array}$ & $\begin{array}{l}\text { The vendor to provide information in its documentation stating which is } \\
\text { the application selected by default within its implementation. }\end{array}$ \\
\hline
\end{tabular}

\subsubsection{Select when Derived PIV Application is Currently Selected}

\begin{tabular}{|l|l|}
\hline Test Assertion & TA-08.03.01.03 \\
\hline Purpose & $\begin{array}{l}\text { Verifies that the Derived PIV Application is not deselected while the } \\
\text { currently selected application is the Derived PIV Application and the } \\
\text { SELECT command is sent with an AID of the Derived PIV Application. } \\
\\
\text { The security status remains unchanged in this case. }\end{array}$ \\
\hline DTR(s) & $\begin{array}{l}\text { - DTR-06.02.02.09 } \\
\text { - DTR-06.03.01.01 }\end{array}$ \\
\hline Vendor & DTR-06.03.01.02 \\
Documentation & None. \\
\hline
\end{tabular}




\begin{tabular}{|c|c|}
\hline Precondition(s) & $\begin{array}{l}\text { - A token with the Derived PIV Application is inserted into an } \\
\text { appropriate token reader. } \\
\text { - Suitable drivers have been loaded between the test system and an } \\
\text { instance of the reader. } \\
\text { - The Derived PIV Application Password's retry counter is not } 0 \text {. }\end{array}$ \\
\hline Test Scenario & 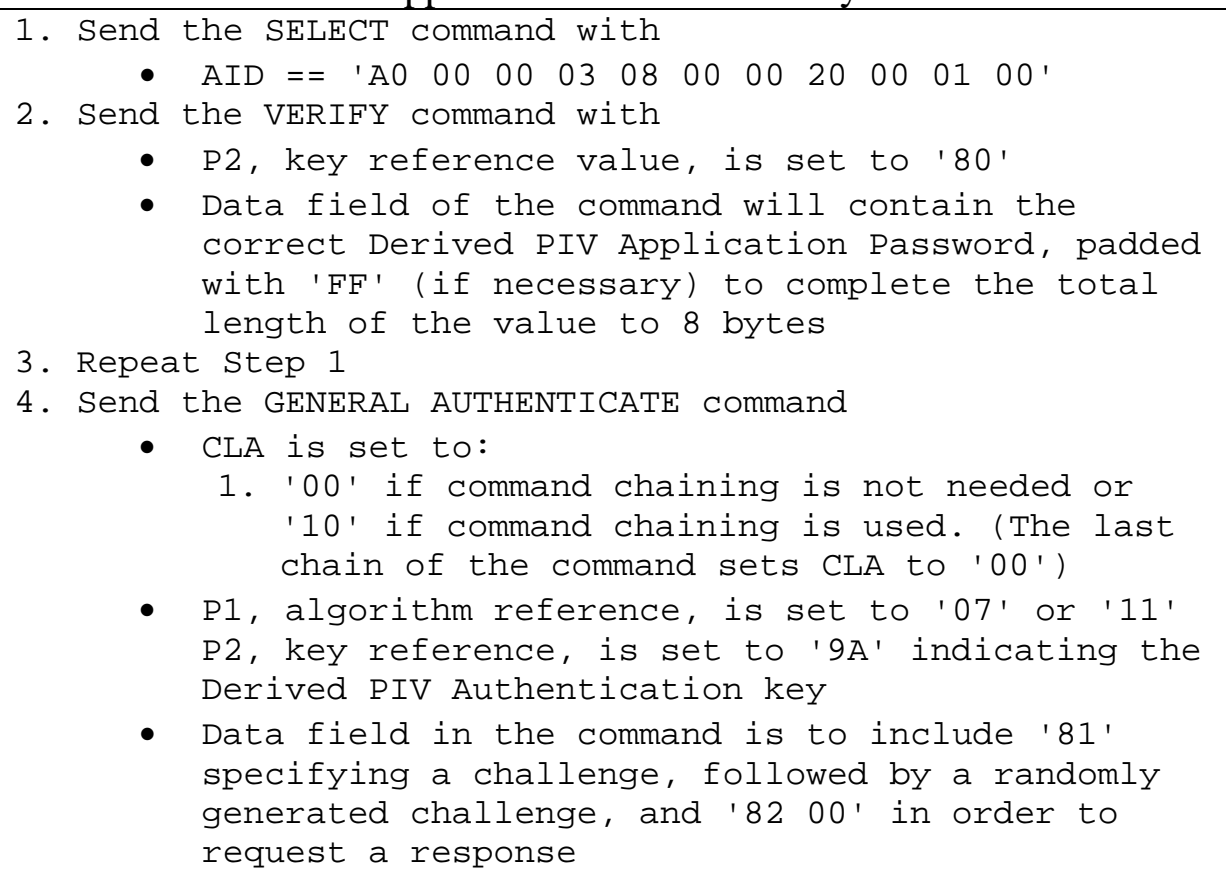 \\
\hline Expected Result(s) & $\begin{array}{l}\text { 1. From Step 1, the command returns the application property template } \\
\text { with the status word ' } 9000 \text { '. } \\
\text { 2. From Step 2, the command returns status word ' } 9000 \text { '. } \\
\text { 3. From Step 3, the command returns the application property template } \\
\text { with the status word ' } 9000 \text { '. } \\
\text { 4. From Step 4, the command returns the signed challenge with status } \\
\text { word ' } 9000 \text { '. }\end{array}$ \\
\hline Postcondition(s) & $\begin{array}{l}\text { The Derived PIV Application is the currently selected application and } \\
\text { the security status of the Derived PIV Application Password is TRUE. }\end{array}$ \\
\hline
\end{tabular}

\subsubsection{Select with an Invalid AID when Derived PIV Application is Currently Selected}

\begin{tabular}{|l|l|}
\hline Test Assertion & TA-08.03.01.04 \\
\hline Purpose & $\begin{array}{l}\text { Verifies that the Derived PIV Application is not deselected while the } \\
\text { currently selected application is the Derived PIV Application and the } \\
\text { SELECT command is sent with an AID that is not supported. }\end{array}$ \\
\hline DTR(s) & $\begin{array}{l}\text { - DTR-06.02.02.09 } \\
\text { - DTR-06.03.01.02 } \\
\text { Vendor }\end{array}$ \\
Documentation & None. \\
\hline
\end{tabular}




\begin{tabular}{|c|c|}
\hline Precondition(s) & $\begin{array}{l}\text { - A token with the Derived PIV Application is inserted into an } \\
\text { appropriate token reader. } \\
\text { - Suitable drivers have been loaded between the test system and an } \\
\text { instance of the reader. } \\
\text { - The Derived PIV Application Password's retry counter is not } 0 \text {. }\end{array}$ \\
\hline Test Scenario & 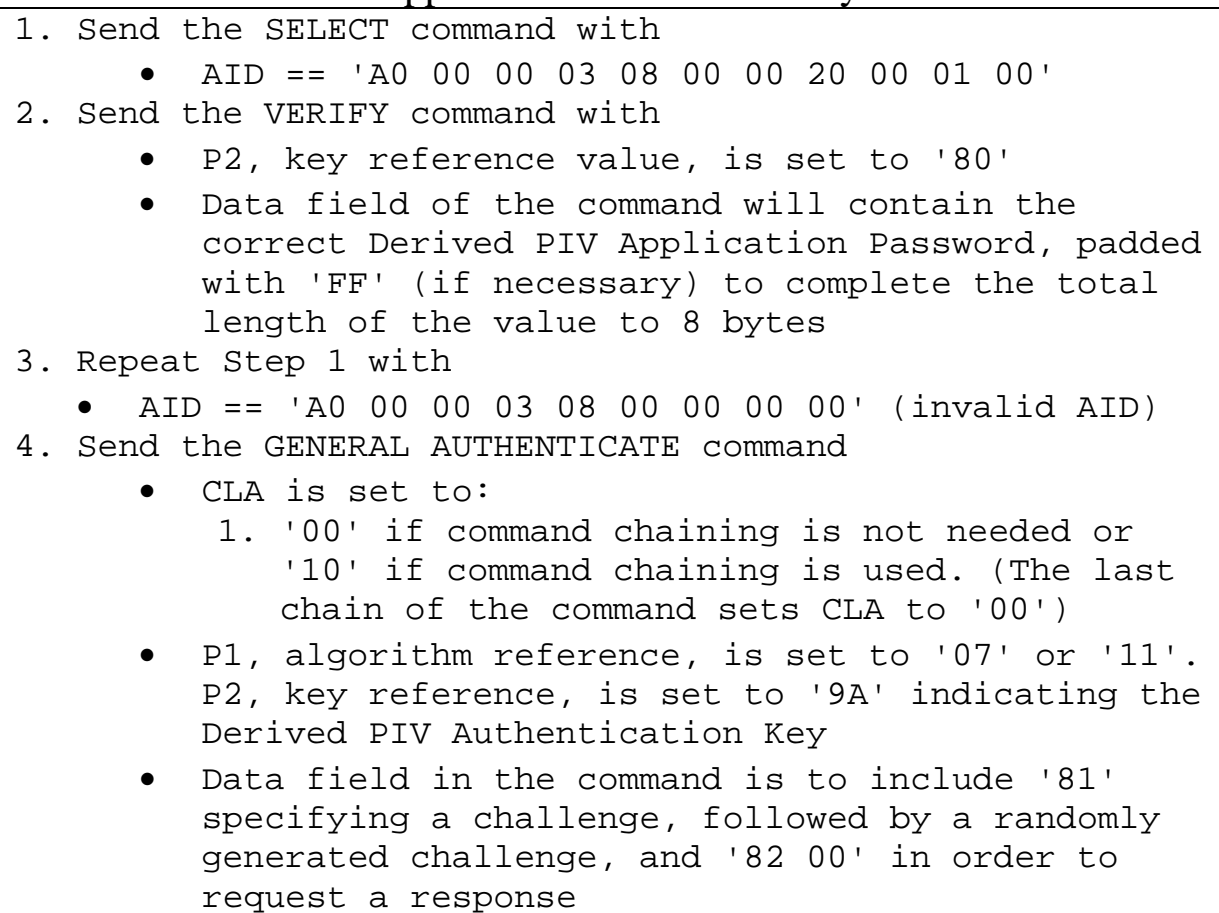 \\
\hline Expected Result(s) & $\begin{array}{l}\text { 1. From Step 1, the command returns the application property template } \\
\text { with the status word ' } 9000 \text { '. } \\
\text { 2. From Step 2, the command returns status word ' } 9000 \text { '. } \\
\text { 3. From Step 3, the command returns '6A } 82 \text { ' (application not found). } \\
\text { 4. From Step 4, the command returns the signed challenge with the } \\
\text { status word ' } 9000 \text { '. }\end{array}$ \\
\hline Postcondition(s) & $\begin{array}{l}\text { The Derived PIV Application continues to be the currently selected } \\
\text { application and the application security status of the Derived PIV } \\
\text { Application Password is TRUE. }\end{array}$ \\
\hline
\end{tabular}

\subsubsection{Select with Another Valid AID when Derived PIV Application is Currently Selected}

\begin{tabular}{|l|l|}
\hline Test Assertion & TA-08.03.01.05 \\
\hline Purpose & $\begin{array}{l}\text { Confirms that the Derived PIV Application is deselected when the } \\
\text { currently selected application is the Derived PIV Application and the } \\
\text { SELECT command is sent with another valid AID that is supported. }\end{array}$ \\
\hline DTR(s) & $\bullet$ DTR-06.03.02.08 \\
\hline $\begin{array}{l}\text { Vendor } \\
\text { Documentation }\end{array}$ & $\begin{array}{l}\text { The vendor to provide information in its documentation validating } \\
\text { compliance with this requirement. }\end{array}$ \\
\hline
\end{tabular}




\subsubsection{GET DATA Command}

\subsubsection{Get Data for the Various Derived PIV Data Objects}

\begin{tabular}{|c|c|}
\hline Test Assertion & TA-08.03.02.01 \\
\hline Purpose & $\begin{array}{l}\text { Verifies that the Derived PIV Application accepts the GET DATA } \\
\text { command with the access rule of each container as specified in Table } 2 \\
\text { of [SP800-73], Part } 1 \text { as mapped to [SP800-157]. This test is applicable } \\
\text { to the mandatory and the optional data objects specified in [SP800-157]. }\end{array}$ \\
\hline DTR(s) & $\begin{array}{l}- \text { DTR-06.02.02.09 } \\
-\quad \text { DTR-06.03.01.01 } \\
- \text { DTR-06.03.03.01 } \\
-\quad \text { DTR-06.03.03.02 } \\
-\quad \text { DTR-06.03.03.03 }\end{array}$ \\
\hline $\begin{array}{l}\text { Vendor } \\
\text { Documentation }\end{array}$ & $\begin{array}{l}\text { The vendor to provide information in its documentation stating all the } \\
\text { optional data objects supported. }\end{array}$ \\
\hline Precondition(s) & $\begin{array}{l}\text { - A token with the Derived PIV Application is inserted into an } \\
\text { appropriate token reader. } \\
\text { - Suitable drivers have been loaded between the test system and an } \\
\text { instance of the reader. } \\
\text { - The mandatory and optional data objects supported by the Derived } \\
\text { PIV Application are loaded. }\end{array}$ \\
\hline Test Scenario & 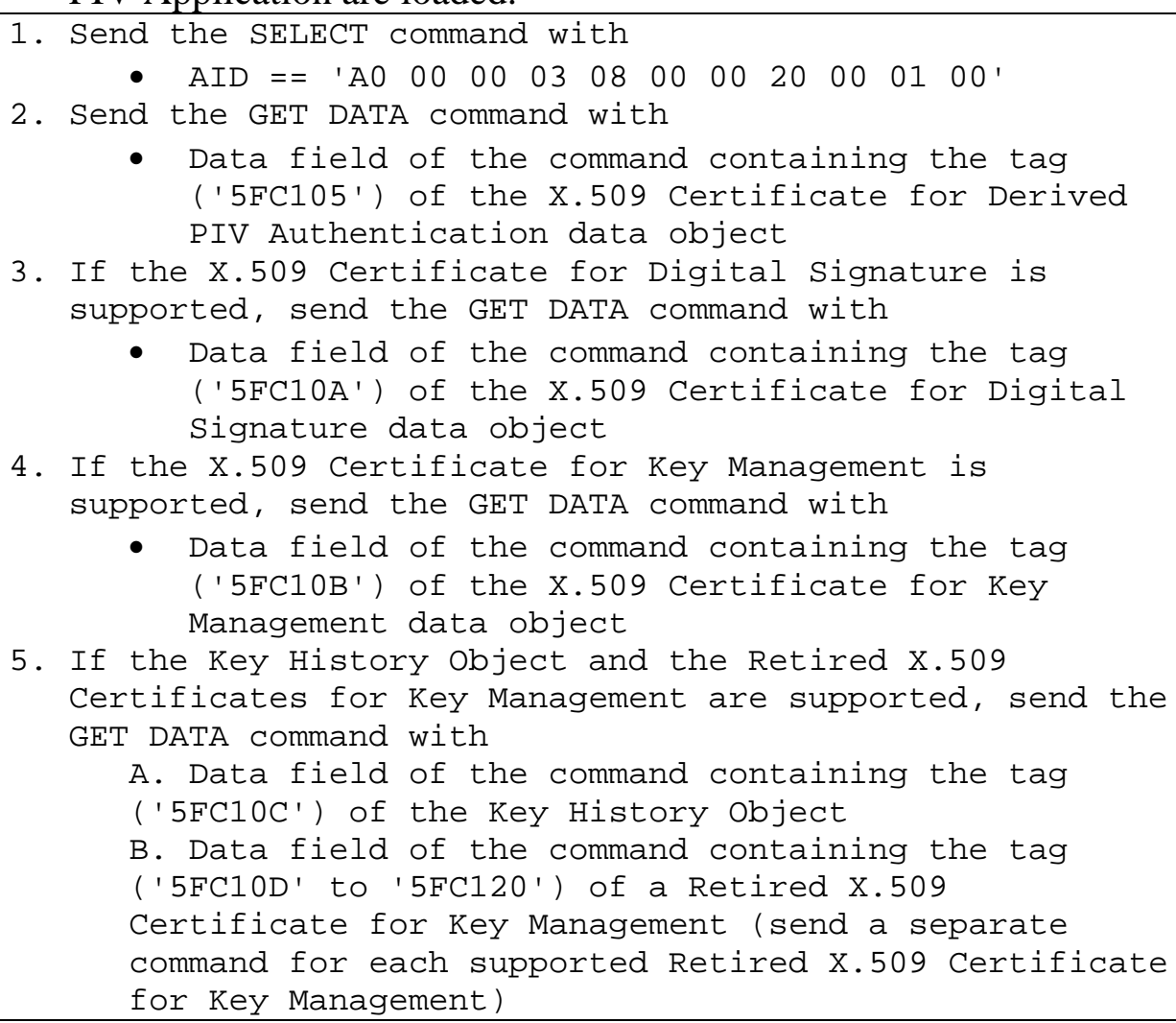 \\
\hline
\end{tabular}




\begin{tabular}{|c|c|}
\hline & $\begin{array}{l}\text { 6. If the Discovery object is supported, send the GET DATA } \\
\text { command with } \\
\text { - Data field of the command containing the tag } \\
\text { ('7E') of the Discovery object } \\
\text { 7. If the Security object is supported, send the GET DATA } \\
\text { command with } \\
\text { - Data field of the command containing the tag } \\
\text { ('5FC106') of the Security Object } \\
\text { 8. Send the GET DATA command with } \\
\text { Data field of the command containing a tag that } \\
\text { does not identify any of the data objects within } \\
\text { the Derived PIV Application. }\end{array}$ \\
\hline Expected Result(s) & $\begin{array}{l}\text { 1. From Step 1, the command returns the application property template } \\
\text { with the status word ' } 9000 \text { '. } \\
\text { 2. For Steps } 2,3,4,5 \mathrm{~A}, 5 \mathrm{~B}, 6 \text { and } 7 \text {, each command returns the } \\
\text { requested data object along with the status word ' } 9000 \text { '. } \\
\text { 3. For Step 8, the command returns status word ' } 6 \mathrm{~A} 82 \text { ' (data object not } \\
\text { found). }\end{array}$ \\
\hline Postcondition(s) & N/A \\
\hline
\end{tabular}

\subsubsection{GENERAL AUTHENTICATE Command}

\subsubsection{Internal Authenticate with the Derived PIV Authentication Key}

\begin{tabular}{|c|c|}
\hline Test Assertion & TA-08.03.03.01 \\
\hline Purpose & $\begin{array}{l}\text { Verifies that the Derived PIV Application responds to the GENERAL } \\
\text { AUTHENTICATE command appropriately when authenticating to the } \\
\text { test toolkit application. }\end{array}$ \\
\hline $\operatorname{DTR}(\mathrm{s})$ & $\begin{array}{l}- \text { DTR-06.02.02.09 } \\
\text { - } \text { DTR-06.03.01.01 } \\
\text { - } \text { DTR-06.03.01.02 } \\
\text { - } \quad \text { DTR-06.03.04.01 } \\
\end{array}$ \\
\hline $\begin{array}{l}\text { Vendor } \\
\text { Documentation }\end{array}$ & None. \\
\hline Precondition(s) & $\begin{array}{l}\text { - A token with the Derived PIV Application is inserted into an } \\
\text { appropriate token reader. } \\
\text { - Suitable drivers have been loaded between the test system and an } \\
\text { instance of the reader. } \\
\text { - The Derived PIV Application Password is recorded. } \\
\text { - The Derived PIV Application Password's retry counter is not } 0 .\end{array}$ \\
\hline Test Scenario & 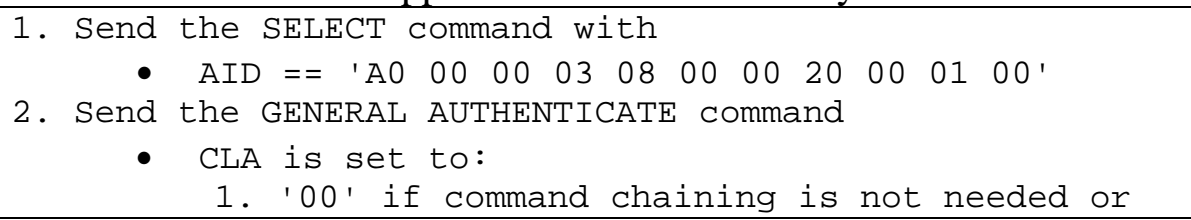 \\
\hline
\end{tabular}




\begin{tabular}{|c|c|}
\hline & $\begin{array}{l}\text { 2. '10' if command chaining is used. (The last } \\
\text { chain of the command sets CLA to 'O0') } \\
\text { - P1, algorithm reference, is set to ' } \odot 7 \text { ' or '11' } \\
\text { - P2, key reference, is set to '9A' (the Derived } \\
\text { PIV Authentication key) } \\
\text { Data field in the command is to include ' } 81 \text { ' } \\
\text { specifying a challenge, followed by a randomly } \\
\text { generated challenge, and '82 } 00^{\prime} \text { in order to } \\
\text { request a response } \\
\text { 3. Send the VERIFY command with } \\
\text { - P2, key reference value, is set to ' } 80 \text { ' } \\
\text { Data field of the command will contain the } \\
\text { correct Derived PIV Application Password, padded } \\
\text { with 'FF' (if necessary) to complete the total } \\
\text { length of the value to } 8 \text { bytes }\end{array}$ \\
\hline Expected Result(s) & $\begin{array}{l}\text { 1. From Step 1, the command returns the application property template } \\
\text { with the status word ' } 9000 \text { '. } \\
\text { 2. From Step 2, the command returns status word ' } 6982 \text { ' (security } \\
\text { status not satisfied). } \\
\text { 3. From Step 3, the command returns status word ' } 90 \text { 00'. } \\
\text { 4. From Step 4, the command returns the signed challenge with status } \\
\text { word '90 00'. Verify the signed challenge. }\end{array}$ \\
\hline Postcondition(s) & N/A \\
\hline
\end{tabular}

\subsubsection{Internal Authenticate with the Derived PIV Authentication Key (with an Invalid Algorithm Reference and Data Length)}

\begin{tabular}{|c|c|}
\hline Test Assertion & TA-08.03.03.02 \\
\hline Purpose & $\begin{array}{l}\text { Verifies that the Derived PIV Application responds to the GENERAL } \\
\text { AUTHENTICATE command appropriately when authenticating to the } \\
\text { test toolkit application using an invalid algorithm reference or data } \\
\text { length. }\end{array}$ \\
\hline DTR(s) & $\begin{array}{ll}- & \text { DTR-06.02.02.09 } \\
- & \text { DTR-06.03.01.01 } \\
\text { - } & \text { DTR-06.03.01.02 } \\
\text { - } & \text { DTR-06.03.04.01 } \\
\text { - } & \text { DTR-06.03.04.05 } \\
\end{array}$ \\
\hline $\begin{array}{l}\text { Vendor } \\
\text { Documentation }\end{array}$ & None. \\
\hline Precondition(s) & $\begin{array}{l}\text { - A token with the Derived PIV Application is inserted into an } \\
\text { appropriate token reader. } \\
\text { - Suitable drivers have been loaded between the test system and an } \\
\text { instance of the reader. } \\
\text { - The Derived PIV Application Password is recorded. } \\
\text { - The Derived PIV Application Password's retry counter is not } 0 \text {. }\end{array}$ \\
\hline
\end{tabular}




\begin{tabular}{|c|c|}
\hline Test Scenario & 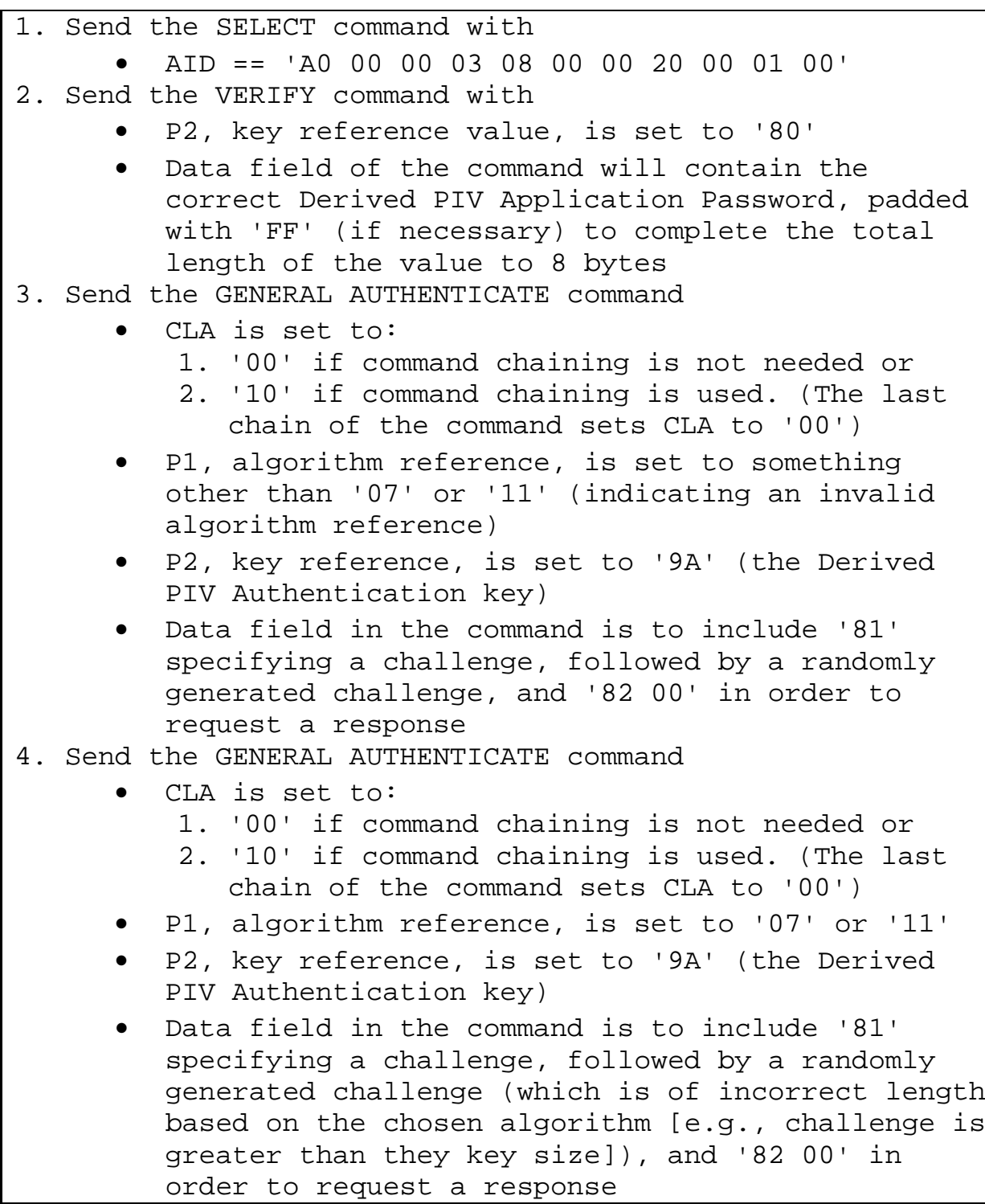 \\
\hline Expected Result(s) & $\begin{array}{l}\text { 1. From Step 1, the command returns the application property template } \\
\text { with the status word ' } 90 \text { 00'. } \\
\text { 2. From Step 2, the command returns status word ' } 90 \text { 00'. } \\
\text { 3. From Step 3, the command returns status word '6A 86' (incorrect } \\
\text { parameter in P1 or P2). } \\
\text { 4. From Step 4, the command returns status word '6A 80' (incorrect } \\
\text { parameter in command data field). }\end{array}$ \\
\hline Postcondition(s) & N/A \\
\hline
\end{tabular}

8.3.3.3 Mutual Authenticate with Derived PIV Token Management Key

\begin{tabular}{|l|l|}
\hline Test Assertion & TA-08.03.03.03 \\
\hline Purpose & $\begin{array}{l}\text { Verifies that the Derived PIV Application responds to the GENERAL } \\
\text { AUTHENTICATE command appropriately when mutually }\end{array}$ \\
\hline
\end{tabular}




\begin{tabular}{|c|c|}
\hline & $\begin{array}{l}\text { authenticating to the test toolkit application using the Derived PIV } \\
\text { Token Management Key (if supported). }\end{array}$ \\
\hline DTR(s) & $\begin{array}{ll} & \text { DTR-06.02.02.09 } \\
\text { - } & \text { DTR-06.03.01.01 } \\
\text { - } & \text { DTR-06.03.04.01 } \\
\text { - } & \text { DTR-06.03.04.05 }\end{array}$ \\
\hline $\begin{array}{l}\text { Vendor } \\
\text { Documentation }\end{array}$ & $\begin{array}{l}\text { The vendor to provide in its documentation whether the Derived PIV } \\
\text { Token Management Key is supported, and if yes, the value of the key. }\end{array}$ \\
\hline Precondition(s) & $\begin{array}{l}\text { - A token with the Derived PIV Application is inserted into an } \\
\text { appropriate token reader. } \\
\text { - Suitable drivers have been loaded between the test system and an } \\
\text { instance of the reader. }\end{array}$ \\
\hline Test Scenario & 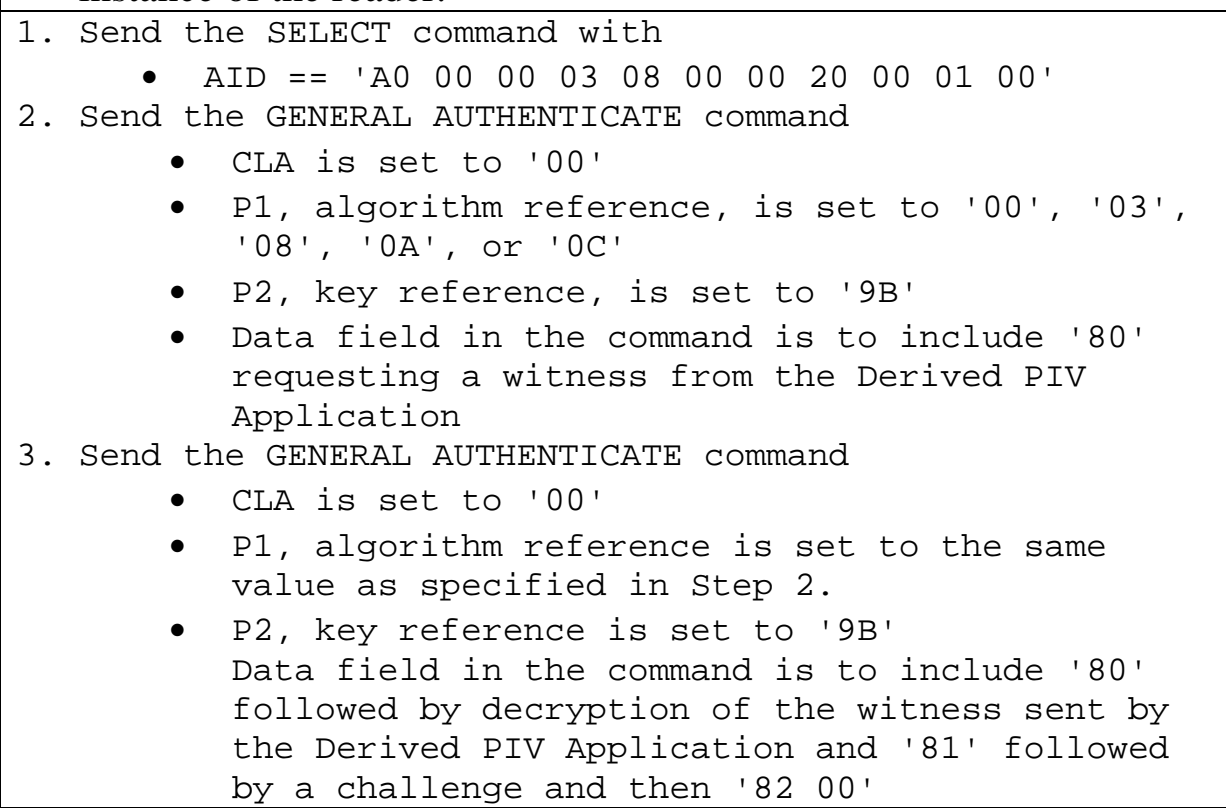 \\
\hline Expected Result(s) & $\begin{array}{l}\text { 1. From Step 1, the command returns the application property template } \\
\text { with the status word ' } 90 \text { 00'. } \\
\text { 2. From Step 2, the command returns with the witness followed by } \\
\text { status word ' } 9000 \text { '. } \\
\text { 3. From Step 3, the Derived PIV Application verifies the decrypted } \\
\text { witness and then responds with encryption of the challenge sent by } \\
\text { Test Toolkit Application followed by status word ' } 9000 \text { '. Decrypt } \\
\text { the encrypted challenge and compare it to the one sent to the token. }\end{array}$ \\
\hline Postcondition(s) & N/A \\
\hline
\end{tabular}

\subsubsection{External Authenticate with Derived PIV Token Management Key}




\begin{tabular}{|c|c|}
\hline Purpose & $\begin{array}{l}\text { Verifies that the Derived PIV Application responds to the GENERAL } \\
\text { AUTHENTICATE command appropriately when externally } \\
\text { authenticating to the test toolkit application using the Derived PIV } \\
\text { Token Management Key (if supported). }\end{array}$ \\
\hline DTR(s) & $\begin{array}{ll} & \text { DTR-06.02.02.09 } \\
\text { - } & \text { DTR-06.03.01.01 } \\
\text { - } & \text { DTR-06.03.04.01 } \\
\text { - } & \text { DTR-06.03.04.05 }\end{array}$ \\
\hline $\begin{array}{l}\text { Vendor } \\
\text { Documentation }\end{array}$ & $\begin{array}{l}\text { The vendor to provide in its documentation whether the Derived PIV } \\
\text { Token Management Key is supported and if yes, the value of the key. }\end{array}$ \\
\hline Precondition(s) & $\begin{array}{l}\text { - A token with the Derived PIV Application is inserted into an } \\
\text { appropriate token reader. } \\
\text { - Suitable drivers have been loaded between the test system and an } \\
\text { instance of the reader. }\end{array}$ \\
\hline Test Scenario & 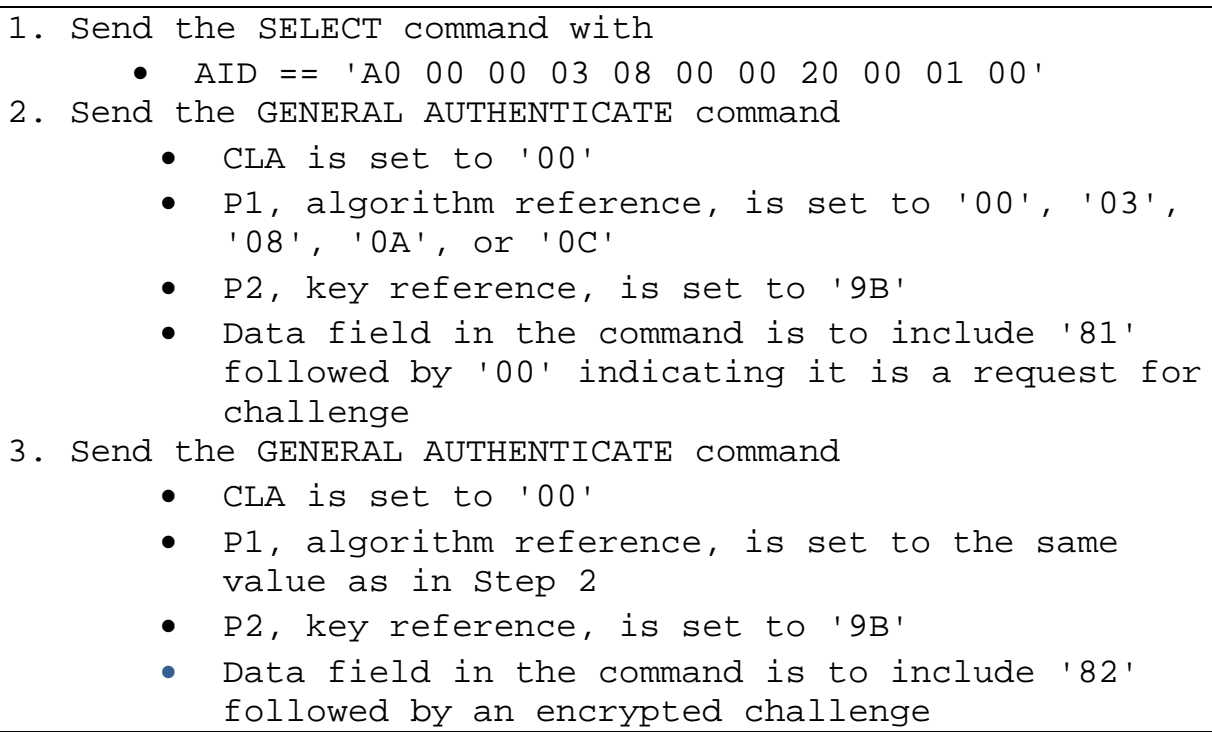 \\
\hline Expected Result(s) & $\begin{array}{l}\text { 1. From Step 1, the command returns the application property template } \\
\text { with the status word ' } 9000 \text { '. } \\
\text { 2. From Step 2, the command returns a challenge followed by status } \\
\text { word ' } 9000 \text { '. } \\
\text { 3. From Step 3, the Test Toolkit Application responds with encryption } \\
\text { of the challenge sent by Derived PIV Application. The token returns } \\
\text { status word ' } 9000 \text { '. }\end{array}$ \\
\hline Postcondition(s) & $\mathrm{N} / \mathrm{A}$ \\
\hline
\end{tabular}

\subsubsection{General Authenticate with the Digital Signature Key}

\begin{tabular}{|l|l|}
\hline Test Assertion & TA-08.03.03.05 \\
\hline Purpose & $\begin{array}{l}\text { Verifies that the Derived PIV Application responds to the GENERAL } \\
\text { AUTHENTICATE command appropriately when signing using the } \\
\text { digital signature key. }\end{array}$ \\
\hline
\end{tabular}




\begin{tabular}{|c|c|}
\hline DTR(s) & $\begin{array}{ll}\text { - } & \text { DTR-06.02.02.09 } \\
\text { - } & \text { DTR-06.03.01.01DTR-06.03.01.02 } \\
\text { - } & \text { DTR-06.03.04.02 } \\
\text { - } & \text { DTR-06.03.04.05 }\end{array}$ \\
\hline $\begin{array}{l}\text { Vendor } \\
\text { Documentation }\end{array}$ & None. \\
\hline Precondition(s) & $\begin{array}{l}\text { - A token with the Derived PIV Application is inserted into an } \\
\text { appropriate token reader. } \\
\text { - Suitable drivers have been loaded between the test system and an } \\
\text { instance of the reader. } \\
\text { - The Derived PIV Application Password is recorded. } \\
\text { - The Derived PIV Application Password's retry counter is not } 0 \text {. }\end{array}$ \\
\hline Test Scenario & 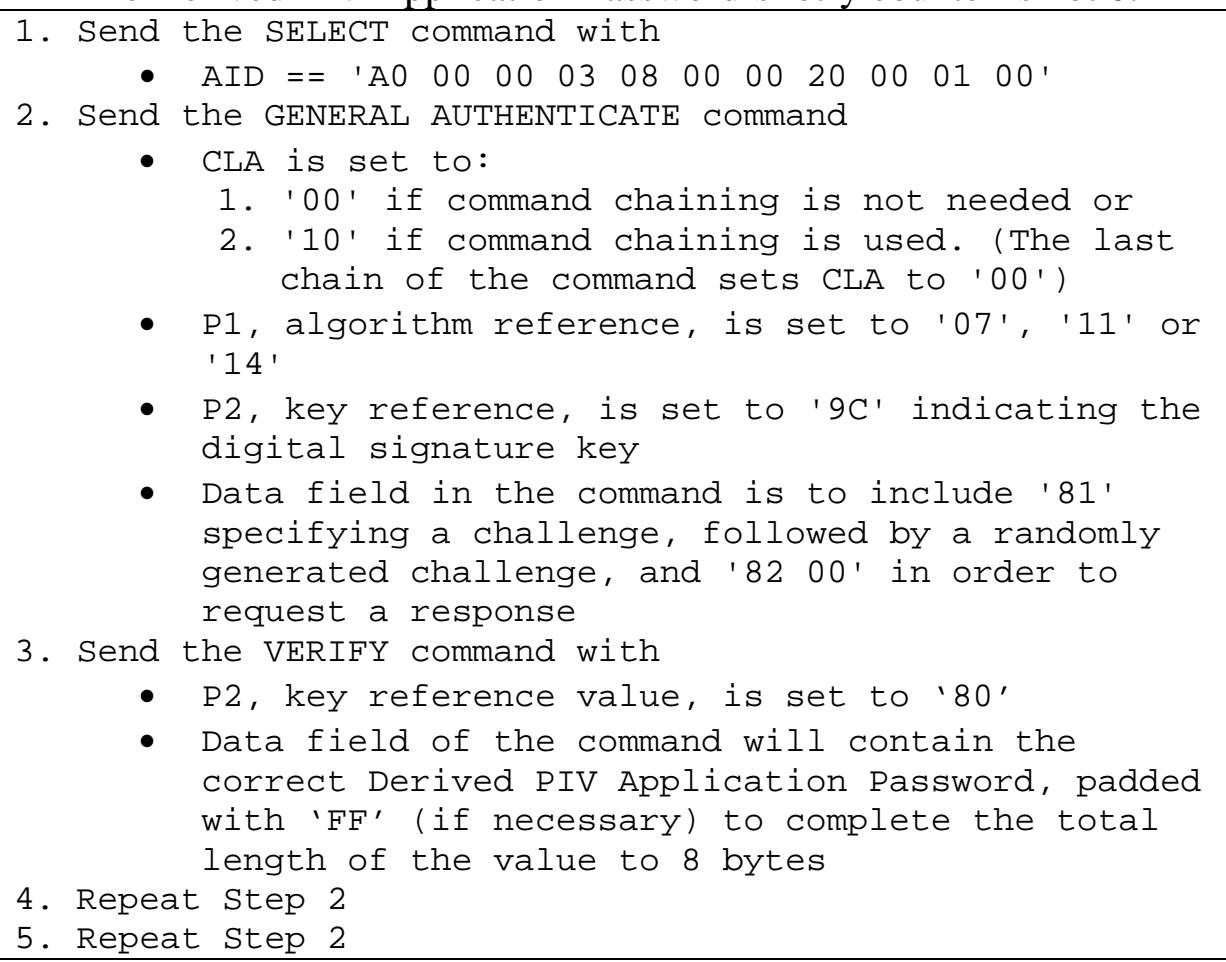 \\
\hline Expected Result(s) & $\begin{array}{l}\text { 1. From Step 1, the command returns the application property template } \\
\text { with the status word ' } 9000 \text { '. } \\
\text { 2. From Step 2, the command returns status word ' } 69 \text { 82' (security } \\
\text { status not satisfied). } \\
\text { 3. From Step 3, the command returns status word ' } 90 \text { 00'. } \\
\text { 4. From Step 4, the command returns the signed challenge with status } \\
\text { word ' } 9000 \text { '. Verify the signature using the public key from the } \\
\text { digital signature certificate and the challenge sent to the token. } \\
\text { 5. From Step 5, the command returns status word '69 82' (security } \\
\text { status not satisfied), since the digital signature key has a "PIN } \\
\text { Always" security condition. }\end{array}$ \\
\hline Postcondition(s) & N/A \\
\hline
\end{tabular}




\subsubsection{Internal Authenticate with the Digital Signature Key (with Invalid Algorithm Reference and Data Length)}

\begin{tabular}{|c|c|}
\hline Test Assertion & TA-08.03.03.06 \\
\hline Purpose & $\begin{array}{l}\text { Verifies that the Derived PIV Application responds to the GENERAL } \\
\text { AUTHENTICATE command appropriately when signing using the } \\
\text { digital signature key with an invalid algorithm reference or data length. }\end{array}$ \\
\hline $\operatorname{DTR}(\mathrm{s})$ & $\begin{array}{ll}\text { - } & \text { DTR-06.02.02.09 } \\
\text { - } & \text { DTR-06.03.01.01 } \\
\text { - } & \text { DTR-06.03.01.02 } \\
\text { - } & \text { DTR-06.03.04.02 } \\
\end{array}$ \\
\hline $\begin{array}{l}\text { Vendor } \\
\text { Documentation }\end{array}$ & None. \\
\hline Precondition(s) & $\begin{array}{l}\text { - A token with the Derived PIV Application is inserted into an } \\
\text { appropriate token reader. } \\
\text { - Suitable drivers have been loaded between the test system and an } \\
\text { instance of the reader. } \\
\text { - The Derived PIV Application Password is recorded. } \\
\text { - The Derived PIV Application Password's retry counter is not } 0 .\end{array}$ \\
\hline Test Scenario & 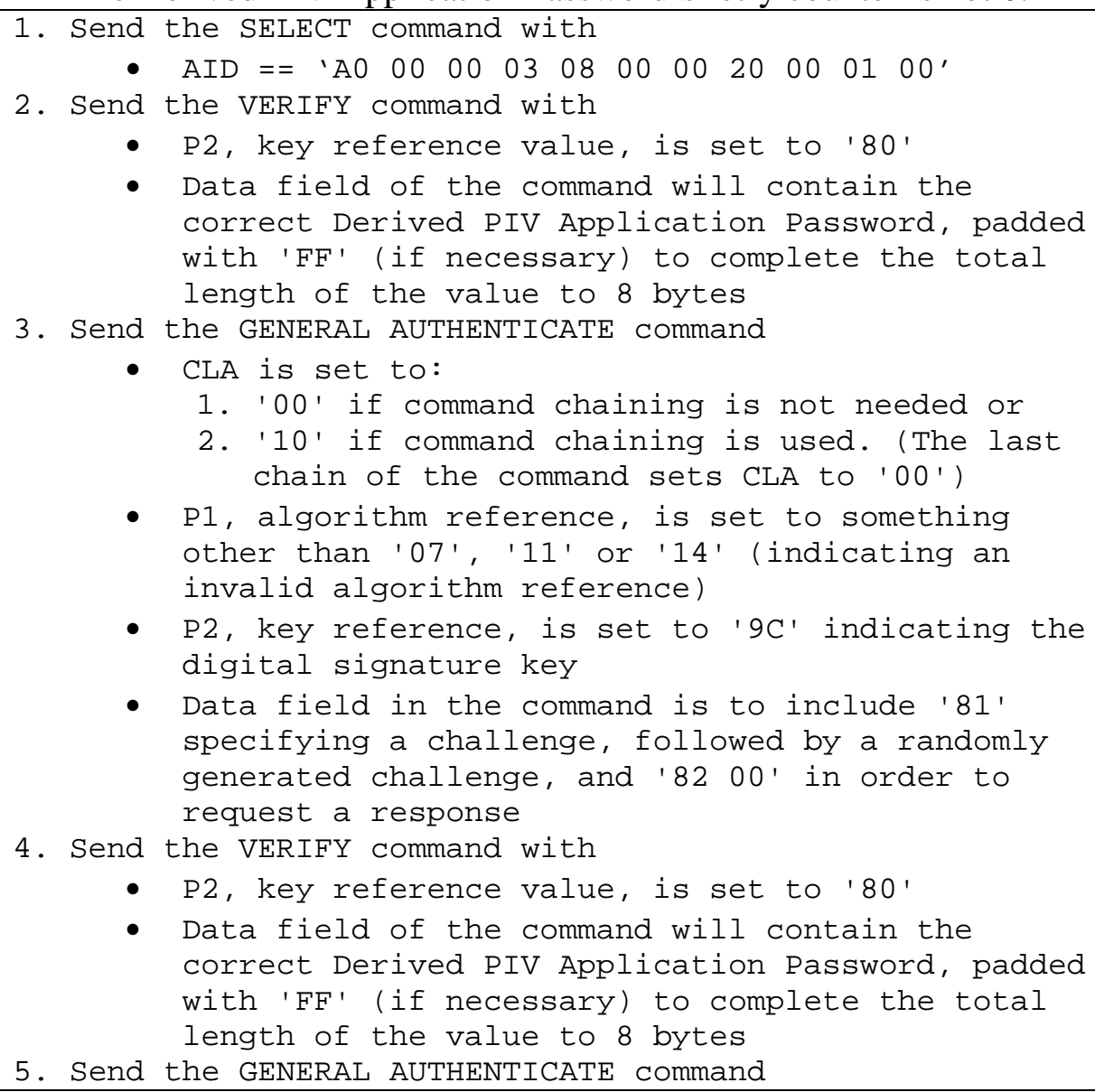 \\
\hline
\end{tabular}




\begin{tabular}{|c|c|}
\hline & $\begin{array}{l}\text { - CLA is set to: } \\
\text { 1. ' } \odot \odot \text { ' if command chaining is not needed or } \\
\text { 2. ' } 10 \text { ' if command chaining is used. (The last } \\
\text { chain of the command sets CLA to ' } 00^{\prime} \text { ') } \\
\text { - } 1 \text { 1, algorithm reference, is set to ' } 07 \text { ', ' } 11 \text { ' or } \\
\text { '14' } \\
\text { P2, key reference, is set to ' } 9 C^{\prime} \text { ' indicating the } \\
\text { digital signature key } \\
\text { - Data field in the command is to include ' } 81 \text { ' } \\
\text { specifying a challenge, followed by a randomly } \\
\text { generated challenge (with an incorrect length } \\
\text { based on the chosen algorithm [e.g.' challenge is } \\
\text { greater than they key size]), and ' } 8200 \text { ' in } \\
\text { order to request a response }\end{array}$ \\
\hline Expected Result(s) & $\begin{array}{l}\text { 1. From Step 1, the command returns the application property template } \\
\text { with the status word ' } 9000 \text { '. } \\
\text { 2. From Step 2, the command returns status word ' } 90 \text { 00'. } \\
\text { 3. From Step 3, the command returns status word '6A 86' (incorrect } \\
\text { parameter in P1 or P2). } \\
\text { 4. From Step 4, the command returns status word ' } 90 \text { 00'. } \\
\text { 5. From Step 5, the command returns status word '6A 80' (incorrect } \\
\text { parameter in command data field). }\end{array}$ \\
\hline Postcondition(s) & N/A \\
\hline
\end{tabular}

\subsubsection{General Authenticate with the Key Management Key}

\begin{tabular}{|c|c|}
\hline Test Assertion & TA-08.03.03.07 \\
\hline Purpose & $\begin{array}{l}\text { Verifies that the Derived PIV Application responds to the GENERAL } \\
\text { AUTHENTICATE command appropriately when using the key } \\
\text { management key. }\end{array}$ \\
\hline DTR(s) & $\begin{array}{ll}- & \text { DTR-06.02.02.09 } \\
\text { - } & \text { DTR-06.03.01.01 } \\
\text { - } & \text { DTR-06.03.01.02 } \\
\text { - } & \text { DTR-06.03.04.03 } \\
\text { - } & \text { DTR-06.03.04.05 } \\
\end{array}$ \\
\hline $\begin{array}{l}\text { Vendor } \\
\text { Documentation }\end{array}$ & None. \\
\hline Precondition(s) & $\begin{array}{l}\text { - A token with the Derived PIV Application is inserted into an } \\
\text { appropriate token reader. } \\
\text { - Suitable drivers have been loaded between the test system and an } \\
\text { instance of the reader. } \\
\text { - The Derived PIV Application Password is recorded. } \\
\text { - The Derived PIV Application Password's retry counter is not } 0 \text {. }\end{array}$ \\
\hline Test Scenario & $\begin{array}{l}\text { 1. Send the SELECT command with } \\
\bullet \text { AID }==\text { 'A } \odot \odot \odot \odot \odot 3 \odot 8 \odot \odot \odot \odot 2 \odot \odot \odot \odot 1 \odot \odot \text { ' } \\
\text { 2. Send the GENERAL AUTHENTICATE command } \\
\qquad \text { CLA is set to: }\end{array}$ \\
\hline
\end{tabular}




\begin{tabular}{|c|c|}
\hline & 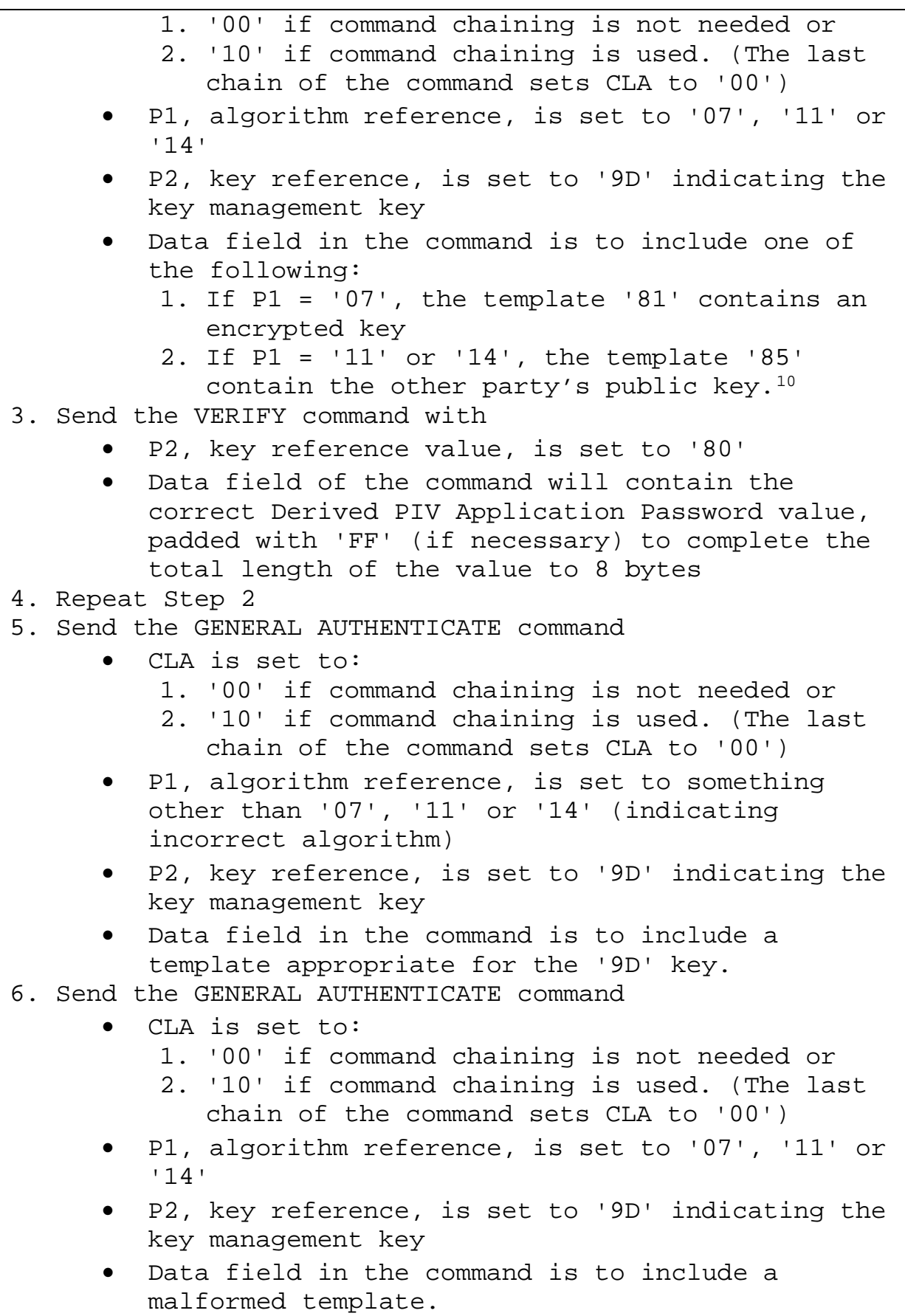 \\
\hline Expected Result(s) & $\begin{array}{l}\text { 1. From Step 1, the command returns the application property template } \\
\text { with the status word ' } 9000 \text { '. } \\
\text { 2. From Step 2, the command returns status word ' } 69 \text { 82' (security } \\
\text { status not satisfied). } \\
\text { 3. From Step 3, the command returns status word ' } 9000 \text { '. } \\
\text { 4. From Step 4, for algorithm reference '07' as P1 value, the command } \\
\text { returns the transported key with status word ' } 90 \text { 00'. Compare the }\end{array}$ \\
\hline
\end{tabular}

${ }^{10}$ Template '85' contains the other party's public key, a point on Curve P-256 or P-384, encoded as '04' || X || Y, without the use of point compression, as described in Section 2.3.3 of [SEC1]. 


\begin{tabular}{|l|l|}
\hline & $\begin{array}{l}\text { test toolkit application's copy of the plaintext key to the one } \\
\text { received in the response from the token. For algorithm reference '11' } \\
\text { or '14' as P1 value, the command returns the shared secret } \mathrm{Z}^{11} \text { with } \\
\text { status word '90 00'. Compare the shared secret computed by the } \\
\text { token with the shared secret computed off token. }\end{array}$ \\
$\begin{array}{l}\text { 5. From Step 5, the command returns status word '6A 86' (incorrect } \\
\text { parameter in P1 or P2). } \\
\text { From Step 6, the command returns status word '6A 80' (incorrect } \\
\text { parameter in command data field). }\end{array}$ \\
\hline Postcondition(s) & N/A \\
\hline
\end{tabular}

\subsubsection{General Authenticate with the Retired Key Management Keys}

\begin{tabular}{|c|c|}
\hline Test Assertion & TA-08.03.03.08 \\
\hline Purpose & $\begin{array}{l}\text { Verifies that the Derived PIV Application responds to the GENERAL } \\
\text { AUTHENTICATE command appropriately when using the retired key } \\
\text { management keys. }\end{array}$ \\
\hline DTR(s) & $\begin{array}{ll} & \text { DTR-06.02.02.03 } \\
\text { - } & \text { DTR-06.03.01.01 } \\
\text { - } & \text { DTR-06.03.04.03 } \\
\text { - } & \text { DTR-06.03.04.05 } \\
\end{array}$ \\
\hline $\begin{array}{l}\text { Vendor } \\
\text { Documentation }\end{array}$ & None. \\
\hline Precondition(s) & $\begin{array}{l}\text { - A token with the Derived PIV Application is inserted into an } \\
\text { appropriate token reader. } \\
\text { - Suitable drivers have been loaded between the test system and an } \\
\text { instance of the reader. } \\
\text { - The Derived PIV Application Password is recorded. } \\
\text { - The Derived PIV Application Password's retry counter is not } 0 .\end{array}$ \\
\hline Test Scenario & 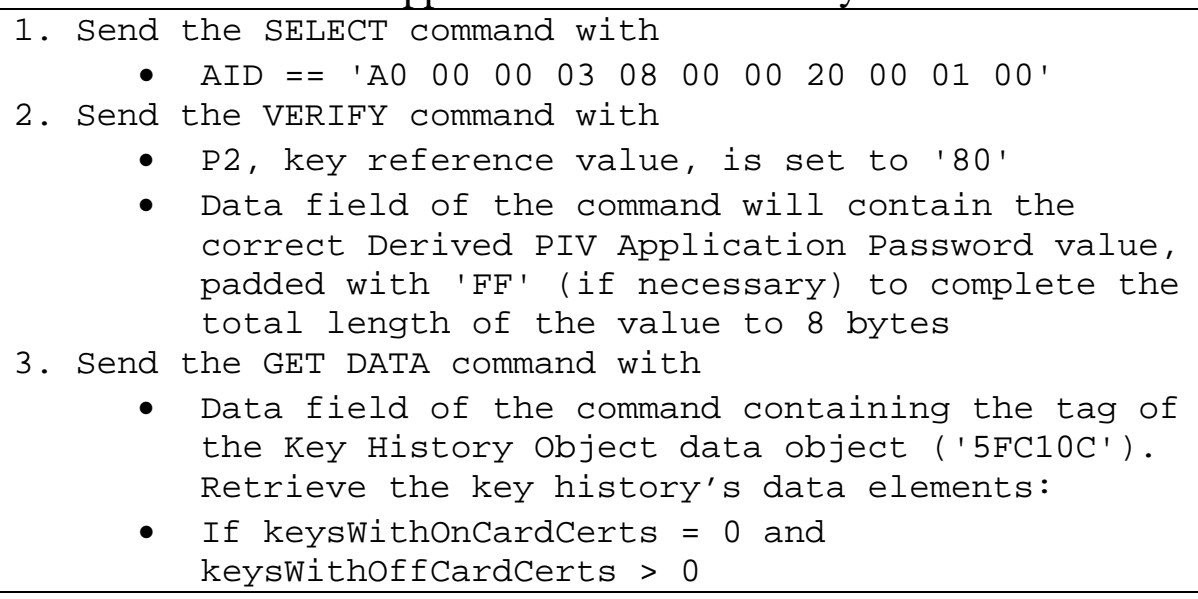 \\
\hline
\end{tabular}

${ }^{11} \mathrm{Z}$ is the $\mathrm{X}$ coordinate of point $\mathrm{P}$ as defined in [SP800-56A], Section 5.7.1.2 


\begin{tabular}{|c|c|}
\hline & 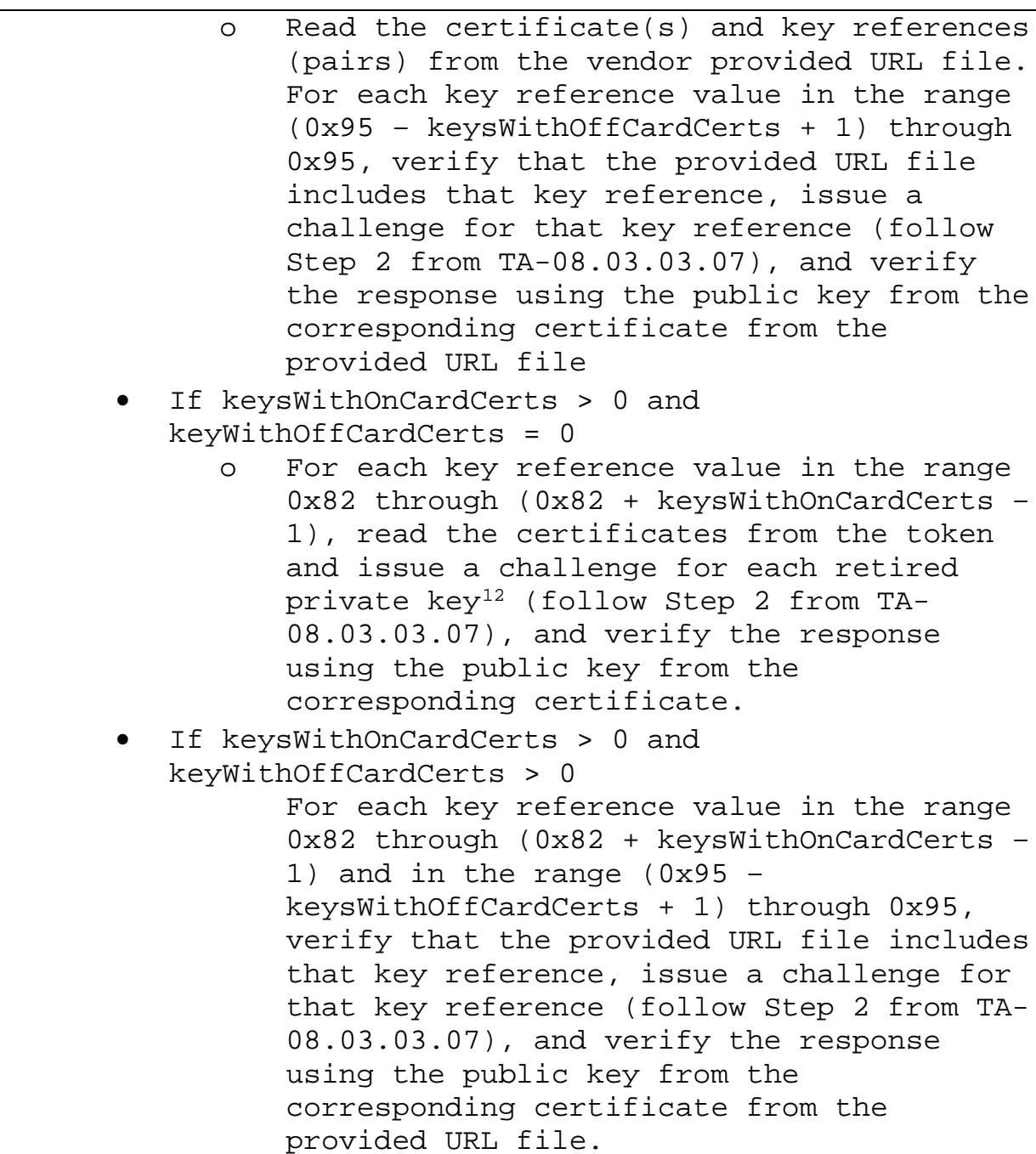 \\
\hline Expected Result(s) & $\begin{array}{l}\text { 1. From Step 1, the command returns the application property template } \\
\text { with the status word ' } 90 \text { 00'. } \\
\text { 2. From Step 2, the command returns status word ' } 90 \text { 00'. } \\
\text { 3. From Step 3, the GET DATA commands return the requested data } \\
\text { along with status word ' } 9000 \text { '. Each GENERAL AUTHENTICATE } \\
\text { command: } \\
\text { a. For key transport (as indicated by algorithm reference '07' as } \\
\text { P1 value), the command returns the transported key with } \\
\text { status word ' } 9000 \text { ' at the end. Compare the test toolkit } \\
\text { application's copy of the plaintext key to the one received in } \\
\text { the response from the token. } \\
\text { b. For ECDH, (as indicated by algorithm reference '11' or '14' } \\
\text { as P1 value), the command returns the shared secret Z with } \\
\text { status word ' } 90 \text { 00'. Compare the shared secret computed by } \\
\text { the token with the shared secret computed off token. }\end{array}$ \\
\hline Postcondition(s) & ( \\
\hline
\end{tabular}

${ }^{12}$ See Table 7 of [SP800-73], Part 1 for the association of certificate BER-TLV tags to corresponding key reference values. 


\subsubsection{Internal Authenticate with an Invalid Key Reference}

\begin{tabular}{|c|c|}
\hline Test Assertion & TA-08.03.03.09 \\
\hline Purpose & $\begin{array}{l}\text { Verifies that the Derived PIV Application responds to the GENERAL } \\
\text { AUTHENTICATE command appropriately when authenticating to the } \\
\text { test toolkit application using an invalid key reference. }\end{array}$ \\
\hline DTR(s) & $\begin{array}{ll}- & \text { DTR-06.02.02.09 } \\
\text { - } & \text { DTR-06.03.01.01 } \\
\text { - } & \text { DTR-06.03.01.02 } \\
\text { - } & \text { DTR-06.03.04.01 } \\
\text { - } & \text { DTR-06.03.04.02 } \\
\text { - } & \text { DTR-06.03.04.03 } \\
\text { - } & \text { DTR-06.03.04.04 } \\
\text { - } & \text { DTR-06.03.04.05 }\end{array}$ \\
\hline $\begin{array}{l}\text { Vendor } \\
\text { Documentation }\end{array}$ & None. \\
\hline Precondition(s) & $\begin{array}{l}\text { - A token with the Derived PIV Application is inserted into an } \\
\text { appropriate token reader. } \\
\text { - Suitable drivers have been loaded between the test system and an } \\
\text { instance of the reader. } \\
\text { - The Derived PIV Application Password is recorded. } \\
\text { - The Derived PIV Application Password's retry counter is not } 0 .\end{array}$ \\
\hline Test Scenario & 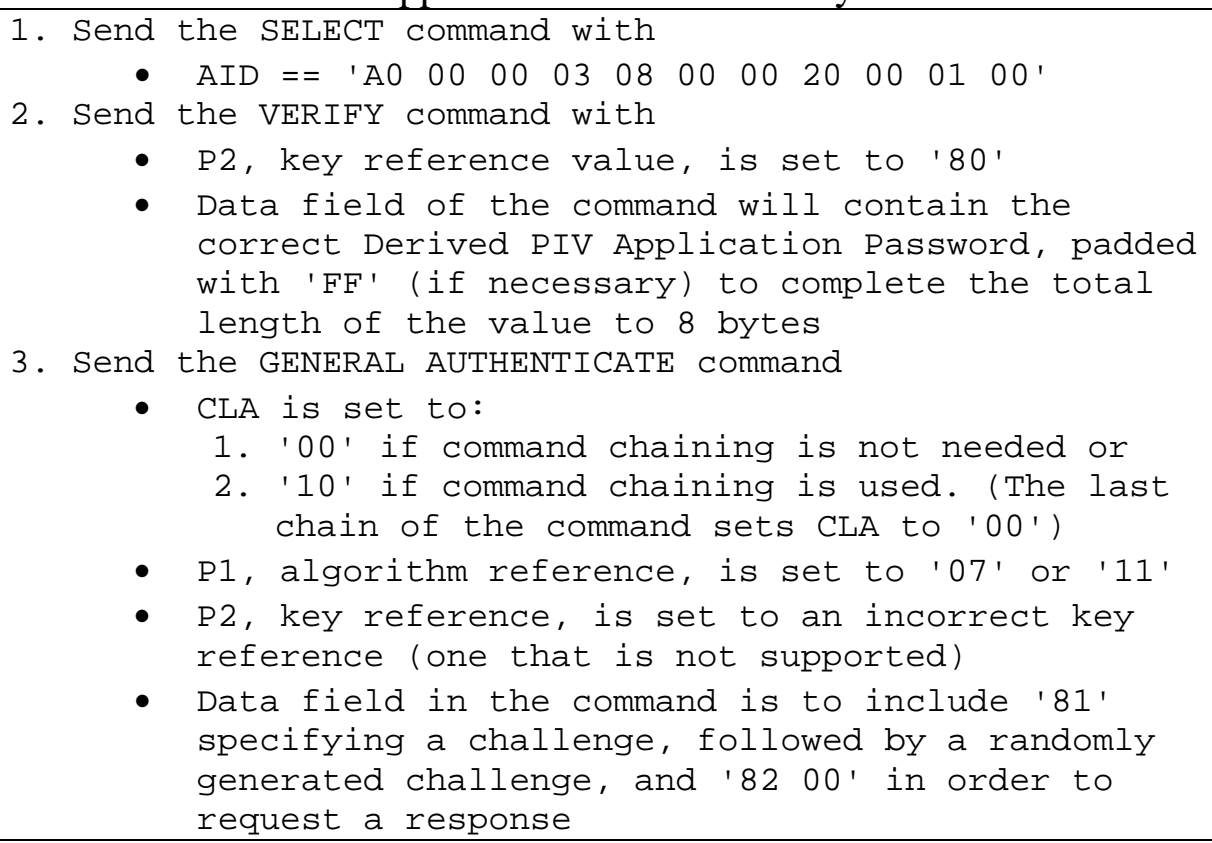 \\
\hline Expected Result(s) & $\begin{array}{l}\text { 1. From Step 1, the command returns the application property template } \\
\text { with the status word ' } 9000 \text { '. } \\
\text { 2. From Step 2, the command returns status word ' } 9000 \text { '. }\end{array}$ \\
\hline
\end{tabular}




\begin{tabular}{|l|l|}
\hline & $\begin{array}{l}\text { 3. From Step 3, the command returns status word '6A 86' (incorrect } \\
\text { parameter in P1 or P2). }\end{array}$ \\
\hline Postcondition(s) & N/A \\
\hline
\end{tabular}

\subsubsection{Support for Command Chaining}

\begin{tabular}{|l|l|}
\hline Test Assertion & TA-08.03.03.10 \\
\hline Purpose & $\begin{array}{l}\text { Confirms that the Derived PIV Application responds to the } \\
\text { GENERAL AUTHENTICATE command and supports command } \\
\text { chaining to permit the uninterrupted transmission of long command } \\
\text { data fields to the Derived PIV Application. }\end{array}$ \\
\hline DTR(s) & $\begin{array}{l}\text { DTR-06.03.04.04 } \\
\text { - DTR-06.03.04.05 }\end{array}$ \\
\hline Vendor Documentation & $\begin{array}{l}\text { The vendor to provide information in its documentation } \\
\text { demonstrating compliance to this requirement. The GET } \\
\text { RESPONSE command is used to return the complete result of the } \\
\text { cryptographic operation. In addition, if a token command other than } \\
\text { the GENERAL AUTHENTICATE command is received by the } \\
\text { Derived PIV Application before the termination of a GENERAL } \\
\text { AUTHENTICATE chain, the Derived PIV Application rolls back } \\
\text { to the state it was in immediately prior to the reception of the first } \\
\text { command in the interrupted chain. }\end{array}$ \\
\hline
\end{tabular}

\subsubsection{VERIFY Command}

\subsubsection{Verify with a Valid Key Reference and the Correct Password}

\begin{tabular}{|c|c|}
\hline Test Assertion & TA-08.03.04.01 \\
\hline Purpose & $\begin{array}{l}\text { Verifies that the Derived PIV Application responds to the VERIFY } \\
\text { command with a valid key reference and sets the security status } \\
\text { appropriately when the correct password is provided. }\end{array}$ \\
\hline DTR(s) & $\begin{array}{l}\text { - } \quad \text { DTR-06.02.02.09 } \\
\text { - } \text { DTR-06.02.02.13 } \\
\text { - } \text { DTR-06.03.01.01 } \\
\text { - } \quad \text { DTR-06.03.01.02 } \\
\end{array}$ \\
\hline Vendor Documentation & None. \\
\hline Precondition(s) & $\begin{array}{l}\text { - A token with the Derived PIV Application is inserted into an } \\
\text { appropriate token reader. } \\
\text { - Suitable drivers have been loaded between the test system and } \\
\text { an instance of the reader. } \\
\text { - The Derived PIV Application Password is recorded. } \\
\text { - The Derived PIV Application Password's retry counter is not } 0 .\end{array}$ \\
\hline
\end{tabular}




\begin{tabular}{|c|c|}
\hline Test Scenario & 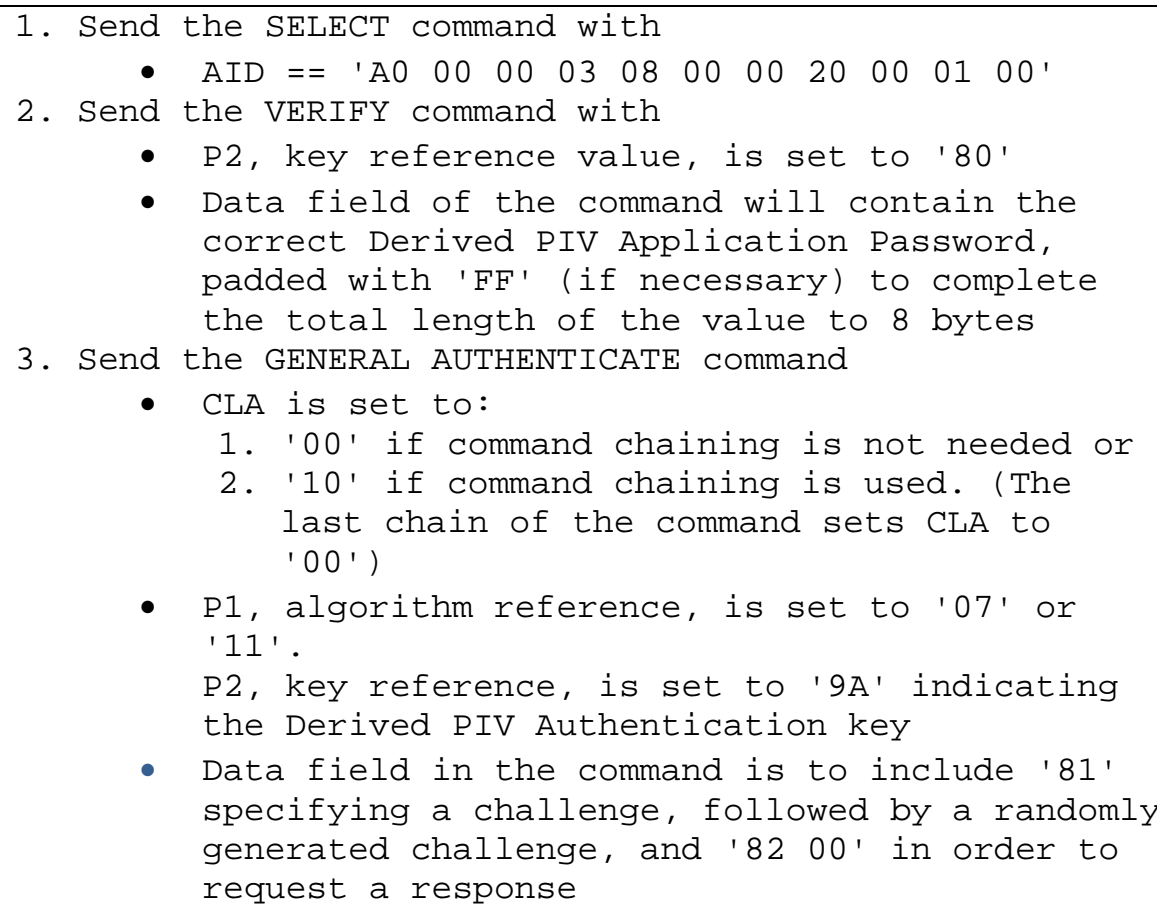 \\
\hline Expected Result(s) & $\begin{array}{l}\text { 1. From Step 1, the command returns the application property } \\
\text { template with the status word ' } 9000 \text { '. } \\
\text { 2. From Step 2, the command returns status word ' } 9000 \text { '. } \\
\text { 3. From Step 3, the command returns the signed challenge with } \\
\text { status word ' } 9000 \text { '. }\end{array}$ \\
\hline Postcondition(s) & e \\
\hline
\end{tabular}

\subsubsection{Verify and Reset Security Status}

\begin{tabular}{|c|c|}
\hline Test Assertion & TA-08.03.04.03 \\
\hline Purpose & $\begin{array}{l}\text { Verifies that the Derived PIV Application responds to the VERIFY } \\
\text { command for resetting the security status with the correct key } \\
\text { reference. }\end{array}$ \\
\hline $\operatorname{DTR}(\mathrm{s})$ & $\begin{array}{ll}- & \text { DTR-06.02.02.09 } \\
- & \text { DTR-06.03.01.01 } \\
- & \text { DTR-06.03.01.02 } \\
- & \text { DTR-06.03.05.01 } \\
- & \text { DTR-06.03.05.05 }\end{array}$ \\
\hline Vendor Documentation & None. \\
\hline Precondition(s) & $\begin{array}{l}\text { - A token with the Derived PIV Application is inserted into an } \\
\text { appropriate token reader. } \\
\text { - Suitable drivers have been loaded between the test system and } \\
\text { an instance of the reader. } \\
\text { - The Derived PIV Application Password is recorded. } \\
\text { - The Derived PIV Application Password's retry counter is not } 0 .\end{array}$ \\
\hline
\end{tabular}




\begin{tabular}{|c|c|}
\hline Test Scenario & 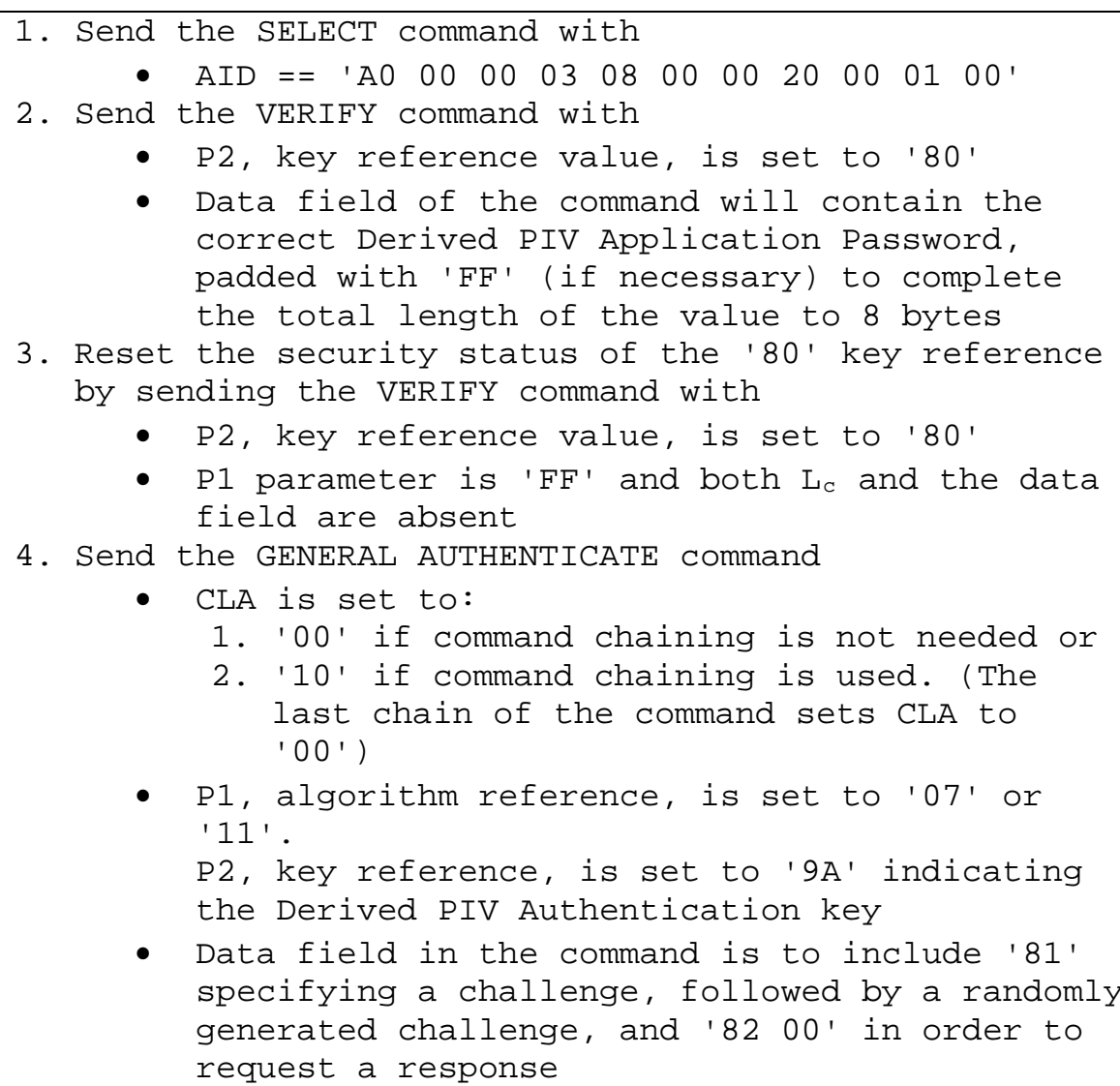 \\
\hline Expected Result(s) & $\begin{array}{l}\text { 1. From Step 1, the command returns the application property } \\
\text { template with the status word ' } 9000 \text { '. } \\
\text { 2. From Step 2, the command returns status word ' } 9000 \text { '. } \\
\text { 3. From Step 3, the command returns status word ' } 9000 \text { '. } \\
\text { 4. From Step 4, the command returns status word ' } 6982 \text { ' (security } \\
\text { status not satisfied). }\end{array}$ \\
\hline Postcondition(s) & $\mathrm{N} / \mathrm{A}$ \\
\hline
\end{tabular}

\subsubsection{Verify with an Incorrect Length and Padding for the Current Password}

\begin{tabular}{|l|l|}
\hline Test Assertion & TA-08.03.04.04 \\
\hline Purpose & $\begin{array}{l}\text { Verifies that the Derived PIV Application responds correctly to the } \\
\text { VERIFY command when the password length and padding } \\
\text { requirements are not met. }\end{array}$ \\
\hline DTR(s) & $\begin{array}{l}\text { - DTR-06.02.02.09 } \\
\text { - DTR-06.02.02.14 }\end{array}$ \\
\hline Vendor Documentation & $\begin{array}{l}\text { The vendor to provide in its documentation the status word returned } \\
\text { by the Derived PIV Application when the password length or } \\
\text { padding requirements are not met. }\end{array}$ \\
\hline Precondition(s) & $\begin{array}{l}\text { A token with the Derived PIV Application is inserted into an } \\
\text { appropriate token reader. }\end{array}$ \\
\hline
\end{tabular}




\begin{tabular}{|c|c|}
\hline & $\begin{array}{l}\text { - Suitable drivers have been loaded between the test system and } \\
\text { an instance of the reader. } \\
\text { - The Derived PIV Application Password is recorded. The } \\
\text { password shall be } 6 \text { bytes in length. } \\
\text { - The Derived PIV Application Password's retry counter is greater } \\
\text { than } 1 .{ }^{13}\end{array}$ \\
\hline Test Scenario & 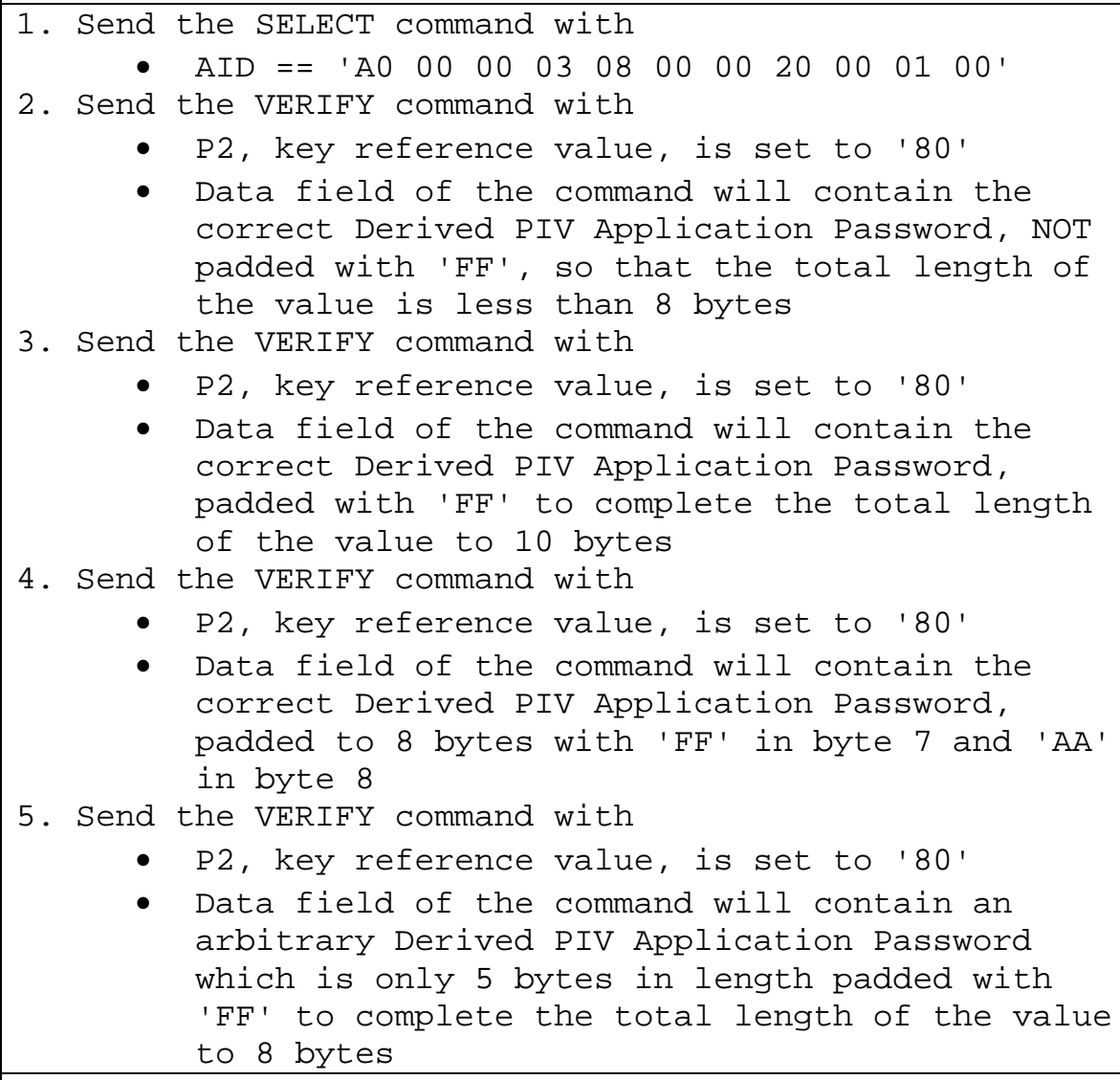 \\
\hline Expected Result(s) & $\begin{array}{l}\text { 1. From Step 1, the command returns the application property } \\
\text { template with the status word ' } 9000 \text { '. } \\
\text { 2. From Step 2, the command returns status word '6A 80' (incorrect } \\
\text { parameter in command data field) or '63 CX' (verification failed, } \\
\text { with X indicating the number of further allowed retries) (verify } \\
\text { the error code supplied matches what is described in vendor } \\
\text { documentation). } \\
\text { 3. From Step 3, the command returns status word '6A 80' (incorrect } \\
\text { parameter command data field) or '63 CX' (verification failed, } \\
\text { with X indicating the number of further allowed retries (verify } \\
\text { the error code supplied matches what is described in vendor } \\
\text { documentation). } \\
\text { 4. From Step 4, the command returns status word '6A 80' (incorrect } \\
\text { parameter in command data field) or '63 CX' (verification failed, }\end{array}$ \\
\hline
\end{tabular}

\footnotetext{
${ }^{13}$ It may be necessary to perform a successful VERIFY command while performing the test scenario in order to keep the Derived PIV Application Password's retry counter from dropping to 0.
} 


\begin{tabular}{|l|l|}
\hline & $\begin{array}{l}\text { with X indicating the number of further allowed retries) (verify } \\
\text { the error code supplied matches what is described in vendor } \\
\text { documentation). }\end{array}$ \\
\begin{tabular}{l} 
5. $\begin{array}{l}\text { From Step 5, the command returns status word '6A 80' (incorrect } \\
\text { parameter in command data field) or '63 CX' (verification failed, } \\
\text { with X indicating the number of further allowed retries) (verify } \\
\text { the error code supplied matches what is described in vendor } \\
\text { documentation). }\end{array}$ \\
\hline Postcondition(s)
\end{tabular} \\
\hline
\end{tabular}

\subsubsection{Verify with an Incorrect Format for the Current Password}

\begin{tabular}{|c|c|}
\hline Test Assertion & TA-08.03.04.05 \\
\hline Purpose & $\begin{array}{l}\text { Verifies that the Derived PIV Application responds appropriately to } \\
\text { the VERIFY command when an incorrectly formatted password is } \\
\text { passed. }\end{array}$ \\
\hline DTR(s) & $\begin{array}{ll} & \text { DTR-06.02.02.09 } \\
\text { - } & \text { DTR-06.02.02.13 } \\
\text { - } & \text { DTR-06.02.02.14 } \\
\text { - } & \text { DTR-06.02.02.15 } \\
\text { - } & \text { DTR-06.03.01.01 } \\
\end{array}$ \\
\hline Vendor Documentation & $\begin{array}{l}\text { The vendor to provide in its documentation the status word returned } \\
\text { by the Derived PIV Application when the password format } \\
\text { requirements are not met. }\end{array}$ \\
\hline Precondition(s) & $\begin{array}{l}\text { - A token with the Derived PIV Application is inserted into an } \\
\text { appropriate token reader. } \\
\text { - Suitable drivers have been loaded between the test system and } \\
\text { an instance of the reader. } \\
\text { - The Derived PIV Application Password is recorded. } \\
\text { - The Derived PIV Application Password's retry counter is greater } \\
\text { than } 1 \text {. }\end{array}$ \\
\hline Test Scenario & 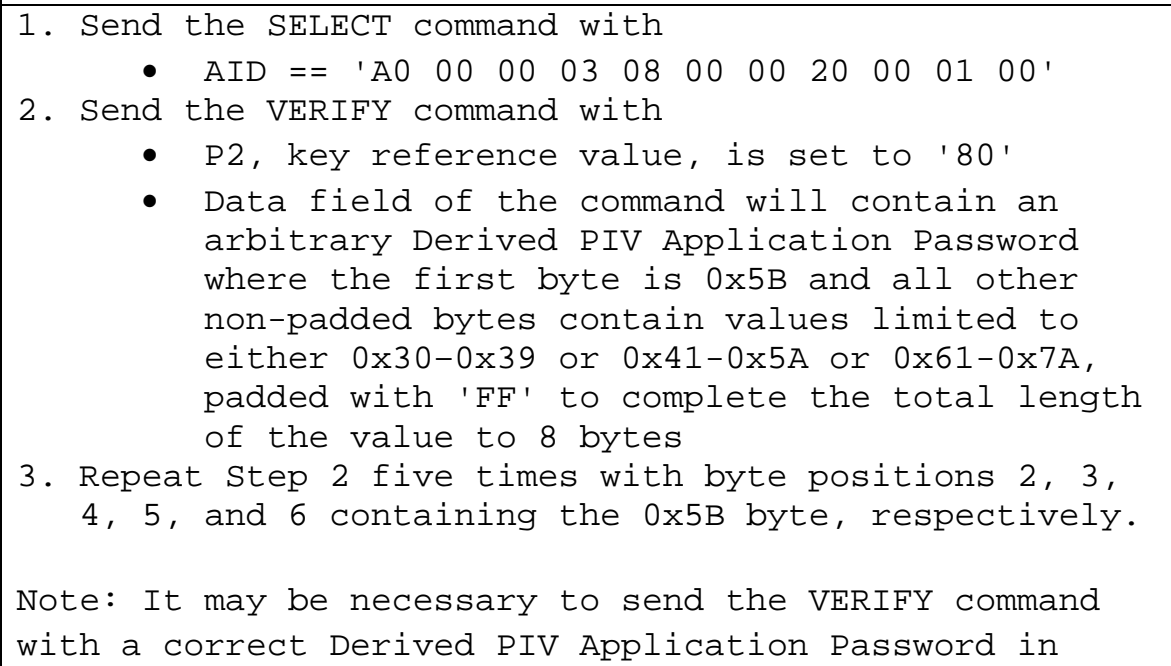 \\
\hline
\end{tabular}




\begin{tabular}{|c|c|}
\hline & $\begin{array}{l}\text { order to prevent the retry counter from decrementing to } \\
\text { zero. }\end{array}$ \\
\hline Expected Result(s) & $\begin{array}{l}\text { 1. From Step 1, the command returns the application property } \\
\text { template with the status word ' } 90 \text { 00'. } \\
\text { 2. From Step 2, the command returns status word '6A } 80 \text { ' (incorrect } \\
\text { parameter in command data field) or '63 CX' (verification failed, } \\
\text { with X indicating the number of further allowed retries). Verify } \\
\text { the error code supplied matches what is described in the } \\
\text { vendor's documentation. } \\
\text { 3. From Step 3, all commands return status word '6A 80' (incorrect } \\
\text { parameter command data field) or '63 CX' (verification failed, } \\
\text { with X indicating the number of further allowed retries). Verify } \\
\text { the error code supplied matches what is described in the } \\
\text { vendor's documentation. }\end{array}$ \\
\hline Postcondition(s) & $\mathrm{N} / \mathrm{A}$ \\
\hline
\end{tabular}

\subsubsection{Verify with an Incorrect Password/Blocking the Derived PIV Application}

\begin{tabular}{|c|c|}
\hline Test Assertion & TA-08.03.04.06 \\
\hline Purpose & $\begin{array}{l}\text { Verifies that the Derived PIV Application is blocked based on the } \\
\text { retry counter when a correctly formatted, but incorrect password is } \\
\text { sent repeatedly using the VERIFY command. }\end{array}$ \\
\hline DTR(s) & $\begin{array}{ll} & \text { DTR-06.02.02.09 } \\
\text { - } & \text { DTR-06.03.01.01 } \\
\text { - } & \text { DTR-06.03.05.02 } \\
\text { - } & \text { DTR-06.03.05.03 } \\
\end{array}$ \\
\hline Vendor Documentation & None. \\
\hline Precondition(s) & $\begin{array}{l}\text { - A token with the Derived PIV Application is inserted into an } \\
\text { appropriate token reader. } \\
\text { - Suitable drivers have been loaded between the test system and } \\
\text { an instance of the reader. } \\
\text { - The Derived PIV Application Password is recorded. } \\
\text { - The Derived PIV Application Password's retry counter is not } 0 .\end{array}$ \\
\hline Test Scenario & $\begin{array}{l}\text { 1. Send the SELECT command with } \\
\text { - AID == 'A๑ } \odot \odot \odot \odot \odot 3 \odot 8 \odot \odot \odot \odot 2 \odot \odot \odot \odot 1 \odot \odot \text { ' } \\
\text { 2. Send the VERIFY command repeatedly, until after the } \\
\text { issuer specified maximum number of password tries is } \\
\text { exceeded with } \\
\text { - P2, key reference value, is set to ' } 8 \odot \text { ' } \\
\text { - Data field of the command will contain an } \\
\text { arbitrary, but correctly formatted, password } \\
\text { value other than what is obtained from the } \\
\text { vendor, padded with 'FF' (if necessary) to } \\
\text { complete the total length of the value to } 8 \\
\text { bytes }\end{array}$ \\
\hline
\end{tabular}




\begin{tabular}{|c|c|}
\hline & $\begin{array}{l}\text { 3. Send the VERIFY command after the issuer specified } \\
\text { maximum number of password tries is exceeded with } \\
\text { - P2, key reference value, is set to ' } 80^{\prime} \\
\text { - Data field of the command will contain the } \\
\text { correct password value, padded with 'FF' (if } \\
\text { necessary) to complete the total length of the } \\
\text { value to } 8 \text { bytes }\end{array}$ \\
\hline Expected Result(s) & $\begin{array}{l}\text { 1. From Step 1, the command returns the application property } \\
\text { template with the status word ' } 9000 \text { '. } \\
\text { 2. From Step 2, the command returns status word ' } 63 \mathrm{CX} \text { ' until the } \\
\text { maximum number of password tries is reached (X indicates the } \\
\text { number of further allowed retries). The command returns status } \\
\text { word '69 83' (authentication method blocked) when the } \\
\text { maximum number of password tries is exceeded. } \\
\text { 3. From Step 3, the command returns status word '69 83' } \\
\text { (authentication method blocked). }\end{array}$ \\
\hline Postcondition(s) & The Derived PIV Application Password’s retry counter is 0. \\
\hline
\end{tabular}

\subsubsection{CHANGE REFERENCE DATA Command}

\subsubsection{Change Reference Data with the Correct Derived PIV Application Password and with the Correct PUK}

\begin{tabular}{|c|c|}
\hline Test Assertion & TA-08.03.05.01 \\
\hline Purpose & $\begin{array}{l}\text { Verifies that the Derived PIV Application can change the current } \\
\text { password with CHANGE REFERENCE DATA command. }\end{array}$ \\
\hline DTR(s) & $\begin{array}{l}\text { - } \quad \text { DTR-06.02.02.09 } \\
\text { - } \text { DTR-06.03.01.01 } \\
\text { - } \text { DTR-06.03.05.06 } \\
\text { - } \quad \text { DTR-06.03.06.01 } \\
\text { - } \text { DTR-06.03.06.03 } \\
\end{array}$ \\
\hline $\begin{array}{l}\text { Vendor } \\
\text { Documentation }\end{array}$ & $\begin{array}{l}\text { The vendor to provide in its documentation the reset retry value of the } \\
\text { Derived PIV Application Password }\end{array}$ \\
\hline Precondition(s) & $\begin{array}{l}\text { - A token with the Derived PIV Application is inserted into an } \\
\text { appropriate token reader. } \\
\text { - Suitable drivers have been loaded between the test system and an } \\
\text { instance of the reader. } \\
\text { - The Derived PIV Application Password and PUK are recorded. } \\
\text { - The Derived PIV Application Password's retry counter and the } \\
\text { PUK's retry counter are not } 0 \text {. }\end{array}$ \\
\hline Test Scenario & 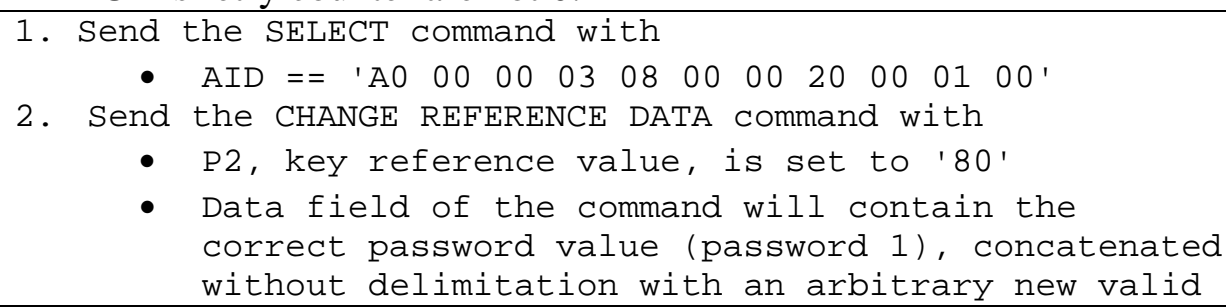 \\
\hline
\end{tabular}




\begin{tabular}{|c|c|}
\hline & 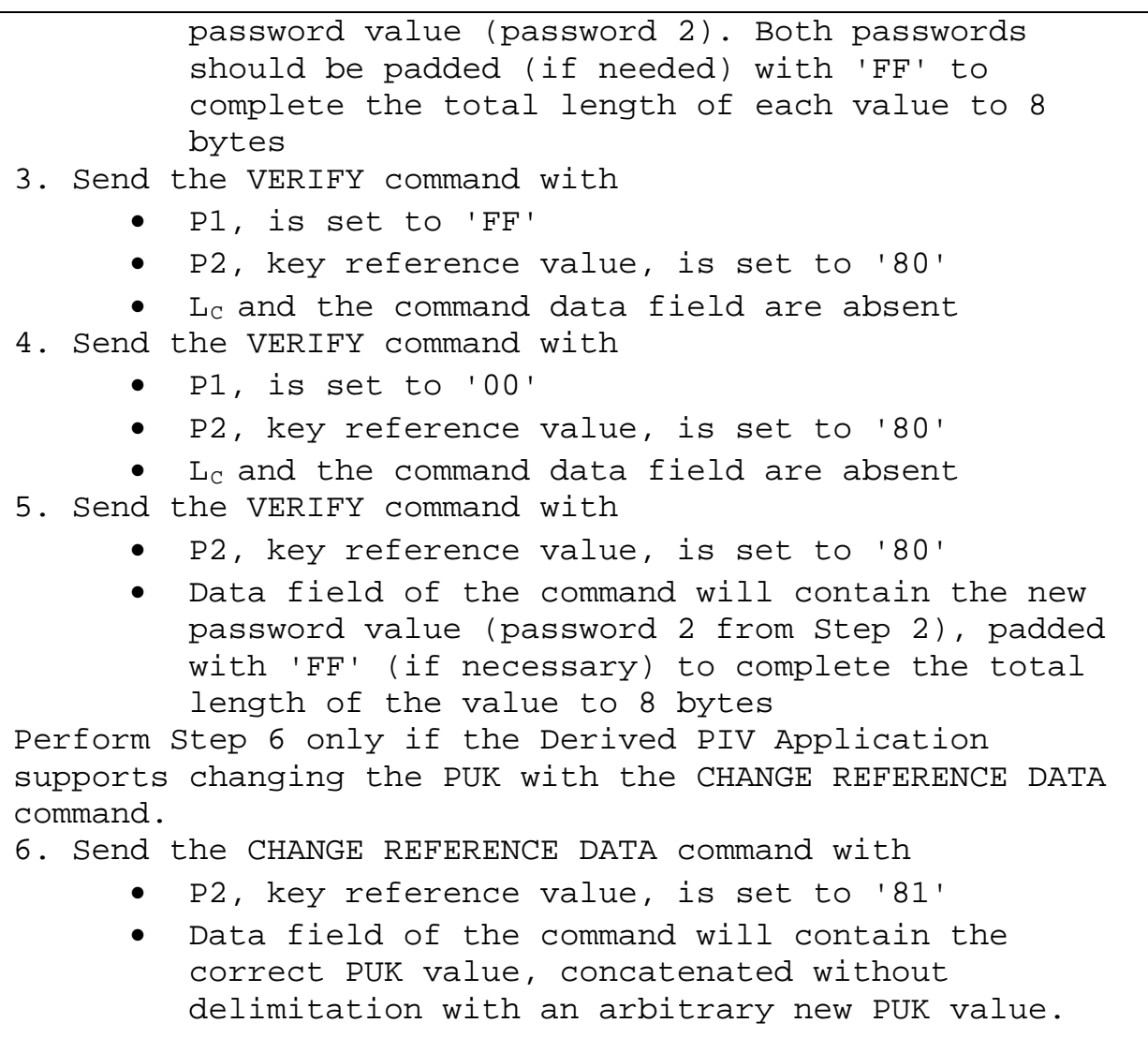 \\
\hline Expected Result(s) & $\begin{array}{l}\text { 1. From Step 1, the command returns the application property template } \\
\text { with the status word ' } 9000 \text { '. } \\
\text { 2. From Step 2, the command returns status word ' } 9000 \text { '. The } \\
\text { password has now been changed. } \\
\text { 3. From Step 3, the command returns status word ' } 90 \text { 00' and the } \\
\text { security status of the PIN is reset. } \\
\text { 4. From Step 4, the command returns status word '63 CX' where X is } \\
\text { equal to the reset retry value of the Derived PIV Application } \\
\text { Password. } \\
\text { 5. Form Step 5, the command returns status word '90 00' } \\
\text { 6. From Step 6, the command returns status word ' } 9000 \text { ' and the PUK } \\
\text { value has been changed. }\end{array}$ \\
\hline Postcondition(s) & $\begin{array}{l}\text { The Derived PIV Application Password and PUK (if supported) are } \\
\text { changed. }\end{array}$ \\
\hline
\end{tabular}

\subsubsection{Change Reference Data with an Invalid Key Reference}

\begin{tabular}{|l|l|}
\hline Test Assertion & TA-08.03.05.02 \\
\hline Purpose & $\begin{array}{l}\text { Verifies that the Derived PIV Application does not change the password } \\
\text { with the CHANGE REFERENCE DATA command with an invalid key } \\
\text { reference. }\end{array}$ \\
\hline
\end{tabular}




\begin{tabular}{|c|c|}
\hline DTR(s) & $\begin{array}{ll}- & \text { DTR-06.02.02.09 } \\
\text { - } & \text { DTR-06.03.01.01 } \\
\text { - } & \text { DTR-06.03.06.02 }\end{array}$ \\
\hline $\begin{array}{l}\text { Vendor } \\
\text { Documentation }\end{array}$ & None. \\
\hline Precondition(s) & $\begin{array}{l}\text { - A token with the Derived PIV Application is inserted into an } \\
\text { appropriate token reader. } \\
\text { - Suitable drivers have been loaded between the test system and an } \\
\text { instance of the reader. } \\
\text { - The Derived PIV Application Password is recorded. } \\
\text { - The Derived PIV Application Password's retry counter is not } 0 .\end{array}$ \\
\hline Test Scenario & 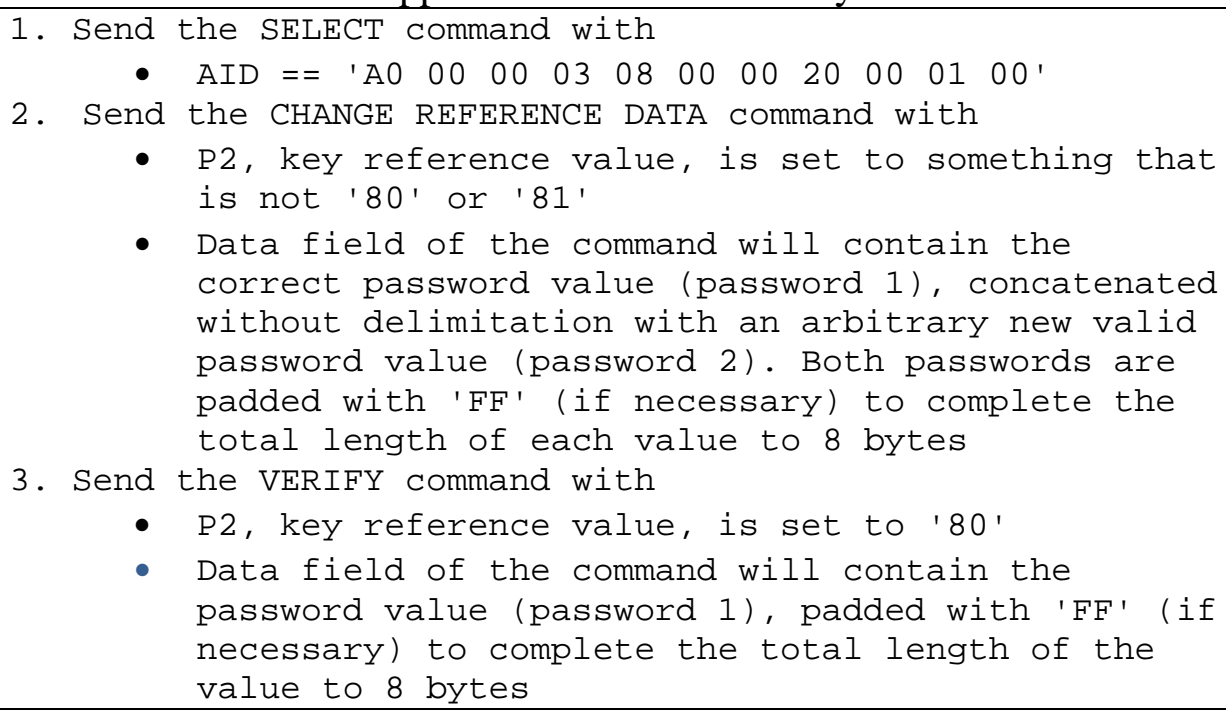 \\
\hline Expected Result(s) & $\begin{array}{l}\text { 1. From Step 1, the command returns the application property template } \\
\text { with the status word ' } 9000 \text { '. } \\
\text { 2. From Step 2, the command returns an error status word. } \\
\text { 3. From Step 3, the command returns status word ' } 90 \text { 00'. }\end{array}$ \\
\hline Postcondition(s) & The original password ('80') is still in effect. \\
\hline
\end{tabular}

\subsubsection{Change Reference Data with an Incorrect Length and Padding for the New Password or with an Incorrect Length of New PUK}

\begin{tabular}{|l|l|}
\hline Test Assertion & TA-08.03.05.03 \\
\hline Purpose & $\begin{array}{l}\text { Verifies that the Derived PIV Application responds appropriately to the } \\
\text { CHANGE REFERENCE DATA command when the length and } \\
\text { padding requirements of the new password are not met or when the } \\
\text { length of the new PUK is incorrect. }\end{array}$ \\
\hline DTR(s) & $\begin{array}{l}\text { - DTR-06.02.02.09 } \\
\text { - DTR-06.02.02.14 }\end{array}$ \\
& - DTR-06.03.01.01 \\
& DTR-06.03.06.05 \\
\hline
\end{tabular}




\begin{tabular}{|c|c|}
\hline & $\begin{array}{ll}\text { - } & \text { DTR-06.03.06.06 } \\
\text { - } & \text { DTR-06.03.06.08 } \\
\end{array}$ \\
\hline $\begin{array}{l}\text { Vendor } \\
\text { Documentation }\end{array}$ & None. \\
\hline Precondition(s) & $\begin{array}{l}\text { - A token with the Derived PIV Application is inserted into an } \\
\text { appropriate token reader. } \\
\text { - Suitable drivers have been loaded between the test system and an } \\
\text { instance of the reader. } \\
\text { - The Derived PIV Application Password and PUK are recorded. } \\
\text { - The Derived PIV Application Password's retry counter and the } \\
\text { PUK's retry counter are not } 0 \text {. }\end{array}$ \\
\hline Test Scenario & 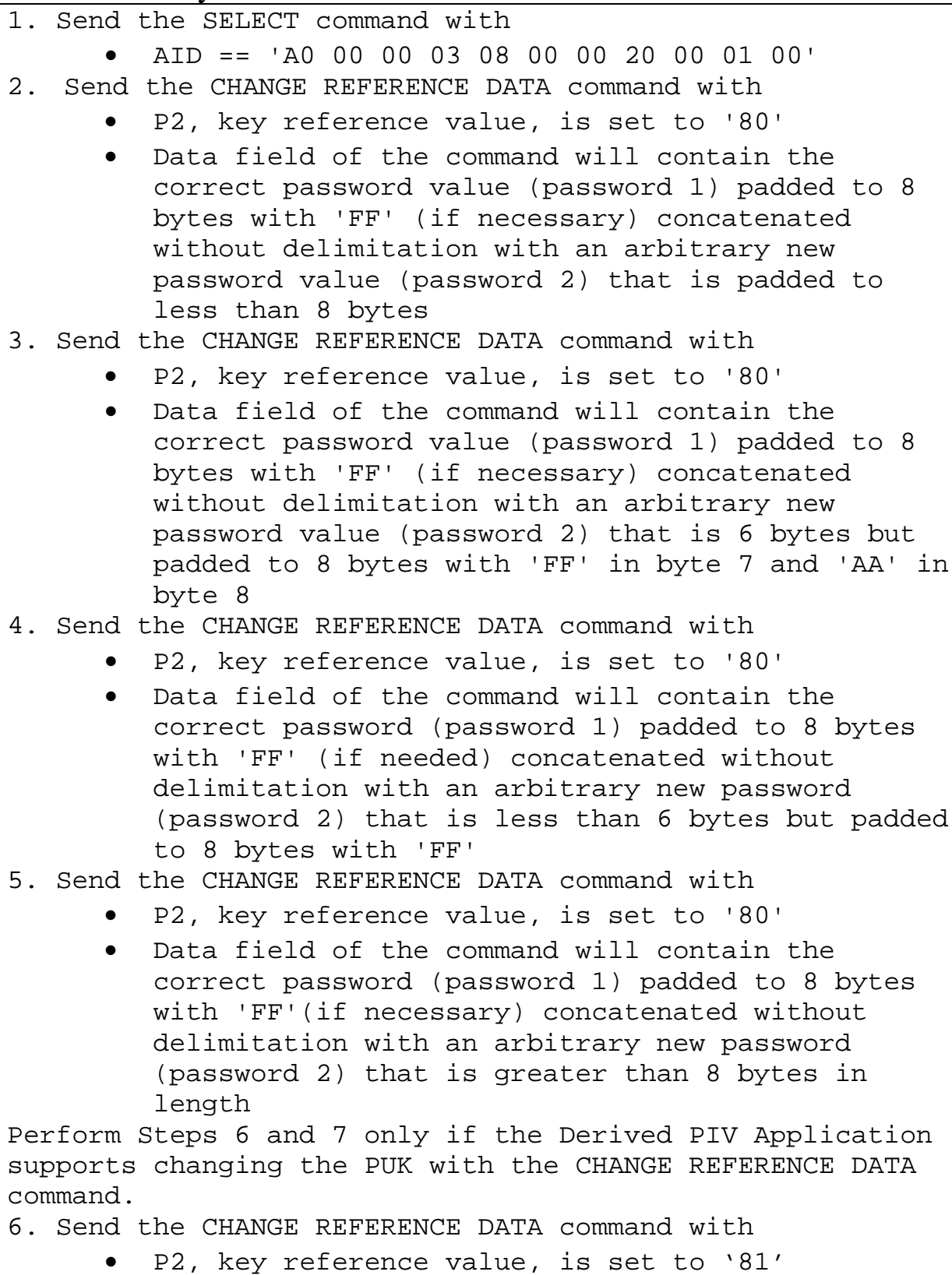 \\
\hline
\end{tabular}




\begin{tabular}{|c|c|}
\hline & $\begin{array}{l}\text { - Data field of the command will contain the } \\
\text { correct PUK value (PUK 1) concatenated without } \\
\text { delimitation with an arbitrary new PUK value (PUK } \\
\text { 2) that is less than } 8 \text { bytes in length } \\
\text { 7. Send the CHANGE REFERENCE DATA command with } \\
\text { - P2, key reference value, is set to '81' } \\
\text { - Data field of the command will contain the } \\
\text { correct PUK (PUK 1) concatenated without } \\
\text { delimitation with an arbitrary new PUK (PUK 3) } \\
\text { that is greater than } 8 \text { bytes in length }\end{array}$ \\
\hline Expected Result(s) & $\begin{array}{l}\text { 1. From Step 1, the command returns the application property template } \\
\text { with the status word ' } 90 \text { 00'. } \\
\text { 2. From Step 2, the command returns status word '6A 80' (incorrect } \\
\text { parameter in command data field). } \\
\text { 3. From Step 3, the command returns status word '6A 80' (incorrect } \\
\text { parameter in command data field). } \\
\text { 4. From Step 4, the command returns status word '6A 80' (incorrect } \\
\text { parameter in command data field). } \\
\text { 5. From Step 5, the command returns status word '6A 80' (incorrect } \\
\text { parameter in command data field). } \\
\text { 6. From Step 6, the command returns status word '6A 80' (incorrect } \\
\text { parameter in the command data field). } \\
\text { 7. From Step 7, the command returns status word '6A 80' (incorrect } \\
\text { parameter in the command data field). }\end{array}$ \\
\hline Postcondition(s) & Neither the password nor the PUK value has changed. \\
\hline
\end{tabular}

\subsubsection{Change Reference Data with an Incorrect Format for the New Password}

\begin{tabular}{|l|l|}
\hline Test Assertion & TA-08.03.05.04 \\
\hline Purpose & Verifies that the Derived PIV Application responds appropriately to the \\
& CHANGE REFERENCE DATA command when the new password \\
does not satisfy the format requirements.
\end{tabular}




\begin{tabular}{|c|c|}
\hline & - $\quad$ The Derived PIV Application Password's retry counter is not 0. \\
\hline Test Scenario & $\begin{array}{l}\text { 1. Send the SELECT command with } \\
\text { 2. Send the CHANGE REFERENCE DATA command with } \\
\text { - P2, key reference value, is set to ' } 8 \odot \text { ' } \\
\text { - Data field of the command will contain the } \\
\text { correct password (password } 1 \text { ) padded to } 8 \text { bytes } \\
\text { with 'FF' (if needed) concatenated without } \\
\text { delimitation with an arbitrary new password value } \\
\text { that contains } 0 \times 5 B \text { in the first byte position, } \\
\text { all other non-padded bytes contain values limited } \\
\text { to either } 0 \times 30-0 \times 39 \text { or } 0 \times 41-0 \times 5 A \text { or } 0 \times 61-0 \times 7 A \\
\text { (password } 2 \text { ). Both passwords should be padded } \\
\text { with 'FF'(as needed) to complete the total length } \\
\text { of each value to } 8 \text { bytes (repeat test five times } \\
\text { with byte positions 2, } 3,4,5 \text {, and } 6 \text { containing } \\
\text { the } 0 \times 5 B \text { byte, respectively) }\end{array}$ \\
\hline Expected Result(s) & $\begin{array}{l}\text { 1. From Step 1, the command returns the application property template } \\
\text { with the status word ' } 9000 \text { '. } \\
\text { 2. From Step 2, each time the command returns status word '6A 80' } \\
\text { (incorrect parameter in command data field). }\end{array}$ \\
\hline Postcondition(s) & Current password is unchanged. \\
\hline
\end{tabular}

\subsubsection{Change Reference Data with an Incorrect Format for the Current Password}

\begin{tabular}{|c|c|}
\hline Test Assertion & TA-08.03.05.05 \\
\hline Purpose & $\begin{array}{l}\text { Verifies that the Derived PIV Application responds appropriately to the } \\
\text { CHANGE REFERENCE DATA command when the current password } \\
\text { format requirements are not met. }\end{array}$ \\
\hline DTR(s) & $\begin{array}{ll}\text { - } & \text { DTR-06.02.02.09 } \\
\text { - } & \text { DTR-06.02.02.14 } \\
\text { - } & \text { DTR-06.03.01.01 } \\
\text { - } & \text { DTR-06.03.06.05 } \\
\text { - } & \text { DTR-06.03.06.08 }\end{array}$ \\
\hline $\begin{array}{l}\text { Vendor } \\
\text { Documentation }\end{array}$ & None. \\
\hline Precondition(s) & $\begin{array}{l}\text { - A token with the Derived PIV Application is inserted into an } \\
\text { appropriate token reader. } \\
\text { - Suitable drivers have been loaded between the test system and an } \\
\text { instance of the reader. } \\
\text { - The Derived PIV Application Password is recorded. } \\
\text { - The Derived PIV Application Password's retry counter is not } 0 \text {. }\end{array}$ \\
\hline Test Scenario & 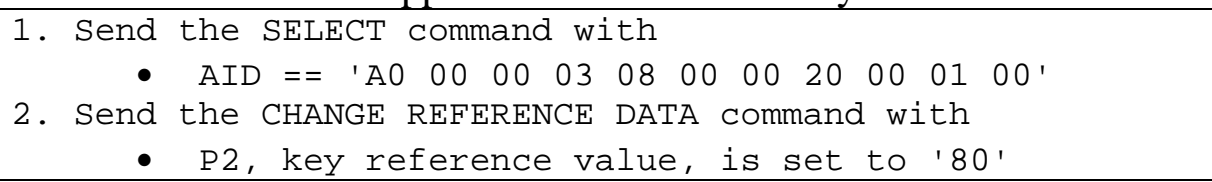 \\
\hline
\end{tabular}




\begin{tabular}{|c|c|}
\hline & $\begin{array}{l}\text { - Data field of the command will contain an } \\
\text { arbitrary password value that contains } 0 \times 5 \mathrm{~B} \text { in } \\
\text { the first byte position, all other non-padded } \\
\text { bytes contain values limited to either } 0 \times 30-0 \times 39 \\
\text { or } 0 \times 41-0 \times 5 \mathrm{~A} \text { or } 0 \times 61-0 \times 7 \mathrm{~A} \text { concatenated without } \\
\text { delimitation with a properly formatted new } \\
\text { password value where all non-padded bytes contain } \\
\text { values limited to either } 0 \times 30-0 \times 39 \text { or } 0 \times 41-0 \times 5 A \\
\text { or } 0 \times 61-0 \times 7 A \text {. Both passwords should be padded } \\
\text { with 'FF'(as needed) to complete the total length } \\
\text { of each value to } 8 \text { bytes. (repeat test five times } \\
\text { with byte positions } 2,3,4,5, \text { and } 6 \text { containing } \\
\text { the } 0 \times 5 B \text { byte, respectively) } \\
\text { Note: In Step } 2 \text { it may be necessary to send the } \\
\text { VERIFy command with a correct Derived PIV } \\
\text { Application Password in order to prevent the } \\
\text { retry counter from decrementing to zero. }\end{array}$ \\
\hline Expected Result(s) & $\begin{array}{l}\text { 1. From Step 1, the command returns the application property template } \\
\text { with the status word ' } 9000 \text { '. } \\
\text { 2. From Step 2, the command returns either status word: } 1 \text { ) ' } 6 \mathrm{~A} 80 \text { ' } \\
\text { (incorrect parameter in command data field) or } 2 \text { ) ' } 63 \mathrm{CX} \text { ' and the } \\
\text { retry counter is decremented by } 1 \text { (where ' } \mathrm{X} \text { ' is the number of tries } \\
\text { remaining). }\end{array}$ \\
\hline Postcondition(s) & Current password is unchanged. \\
\hline
\end{tabular}

\subsubsection{Change Reference Data with a Correctly Formatted but Incorrect Current Password or with a Correctly Formatted but Incorrect PUK}

\begin{tabular}{|c|c|}
\hline Test Assertion & TA-08.03.05.06 \\
\hline Purpose & $\begin{array}{l}\text { Verifies that the Derived PIV Application responds appropriately when } \\
\text { the CHANGE REFERENCE DATA command is sent repeatedly with a } \\
\text { correctly formatted, but incorrect, Derived PIV Application Password or } \\
\text { with a correctly formatted, but incorrect, PUK value. }\end{array}$ \\
\hline DTR(s) & $\begin{array}{ll}\text { - } & \text { DTR-06.02.02.09 } \\
\text { - } & \text { DTR-06.03.01.01 } \\
\text { - } & \text { DTR-06.03.06.04 } \\
\text { - } & \text { DTR-06.03.06.05 } \\
\text { - } & \text { DTR-06.03.06.07 } \\
\text { - } & \text { DTR-06.03.06.09 }\end{array}$ \\
\hline $\begin{array}{l}\text { Vendor } \\
\text { Documentation }\end{array}$ & None. \\
\hline Precondition(s) & $\begin{array}{l}\text { - A token with the Derived PIV Application is inserted into an } \\
\text { appropriate token reader. } \\
\text { - Suitable drivers have been loaded between the test system and an } \\
\text { instance of the reader. } \\
\text { - The Derived PIV Application Password and the PUK are recorded. }\end{array}$ \\
\hline
\end{tabular}




\begin{tabular}{|c|c|}
\hline & $\begin{array}{l}\text { - The reset retry values for the Derived PIV Application Password } \\
\text { and PUK are recorded. } \\
\text { - The Derived PIV Application Password's retry counter and the } \\
\text { PUK's retry counter are not } 0 \text {. }\end{array}$ \\
\hline Test Scenario & 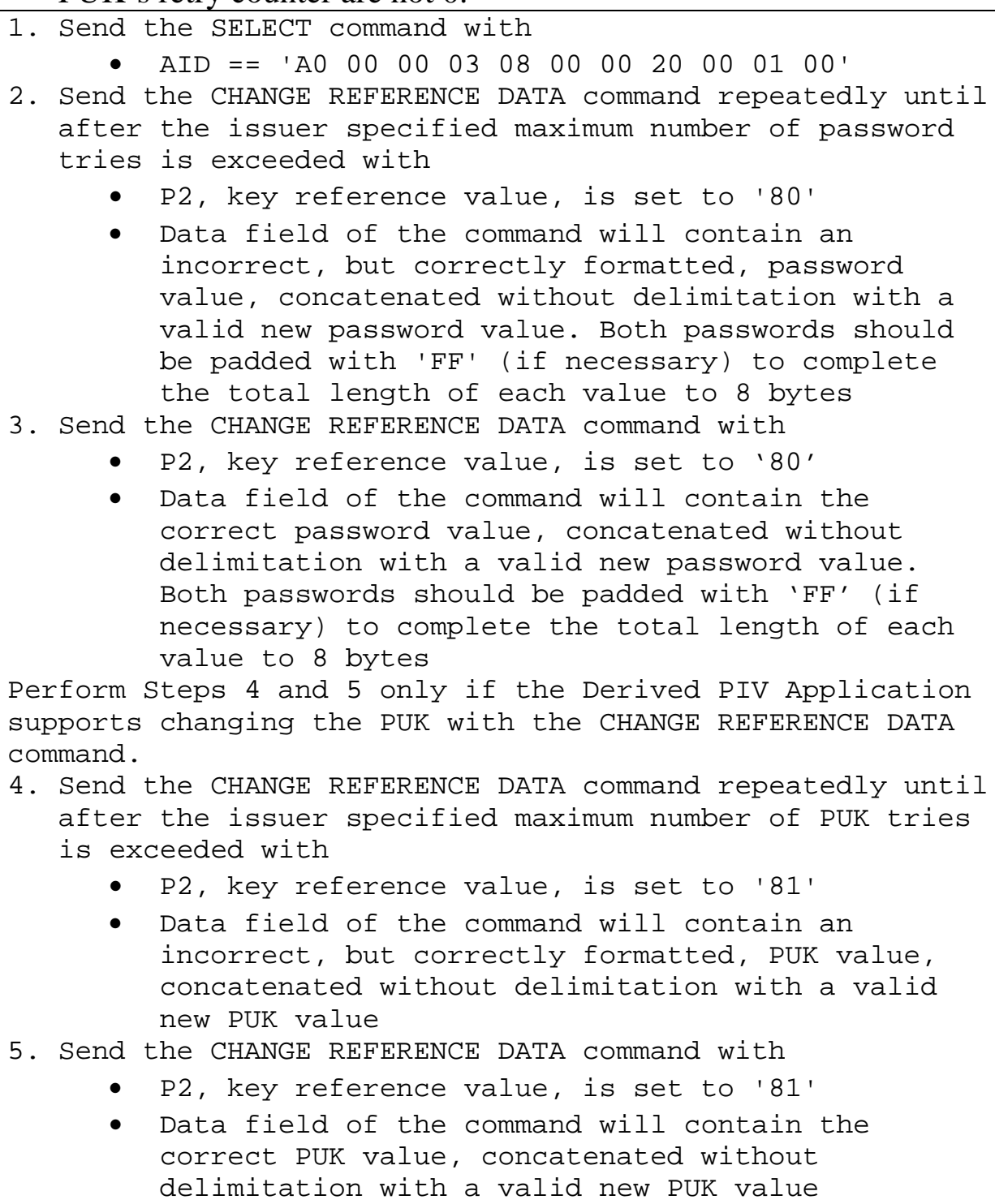 \\
\hline Expected Result(s) & $\begin{array}{l}\text { 1. From Step 1, the command returns the application property template } \\
\text { with the status word ' } 90 \text { 00'. } \\
\text { 2. From Step 2, each time the command returns status word '63 CX' } \\
\text { (where 'X' indicates the number of further allowed retries) and the } \\
\text { retry counter is decremented. The command returns status word '69 } \\
\text { 83' (reference data change operation blocked) when the maximum } \\
\text { number of tries is exceeded. } \\
\text { 3. From Step 3, the command returns status word '69 83' (reference } \\
\text { data change operation blocked). } \\
\text { 4. From Step 4, each time the command returns status word '63 CX' } \\
\text { (where 'X' indicates the number of further allowed retries) and the } \\
\text { retry counter is decremented. The command returns status word '69 }\end{array}$ \\
\hline
\end{tabular}




\begin{tabular}{|l|l|}
\hline & \multicolumn{1}{|c|}{$\begin{array}{l}\text { 83' (reference data change operation blocked) when the maximum } \\
\text { number of tries is exceeded. } \\
\text { 5. From Step 5, the command returns status word '69 83' (reference } \\
\text { data change operation blocked). }\end{array}$} \\
\hline Postcondition(s) & $\begin{array}{l}\text { The Derived PIV Application Password's retry counter and the PUK's } \\
\text { retry counter are 0. }\end{array}$ \\
\hline
\end{tabular}

\subsubsection{RESET RETRY COUNTER Command}

\subsubsection{Reset Retry Counter for the Derived PIV Application Password}

\begin{tabular}{|c|c|}
\hline Test Assertion & TA-08.03.06.01 \\
\hline Purpose & $\begin{array}{l}\text { Verifies that the Derived PIV Application changes the password with } \\
\text { the RESET RETRY COUNTER command when the PUK and } \\
\text { command format are correct. }\end{array}$ \\
\hline DTR(s) & $\begin{array}{ll} & \text { DTR-06.02.02.09 } \\
\text { - } & \text { DTR-06.03.01.01 } \\
\text { - } & \text { DTR-06.03.07.01 } \\
\text { - } & \text { DTR-06.03.07.08 }\end{array}$ \\
\hline $\begin{array}{l}\text { Vendor } \\
\text { Documentation }\end{array}$ & None. \\
\hline Precondition(s) & $\begin{array}{l}\text { - A token with the Derived PIV Application is inserted into an } \\
\text { appropriate token reader. } \\
\text { - Suitable drivers have been loaded between the test system and an } \\
\text { instance of the reader. } \\
\text { - The PUK is recorded. } \\
\text { - The reset retry value for the Derived PIV Application Password is } \\
\text { recorded. } \\
\text { - The Derived PIV Application Password's retry counter and the } \\
\text { PUK's retry counter are not } 0 \text {. }\end{array}$ \\
\hline Test Scenario & 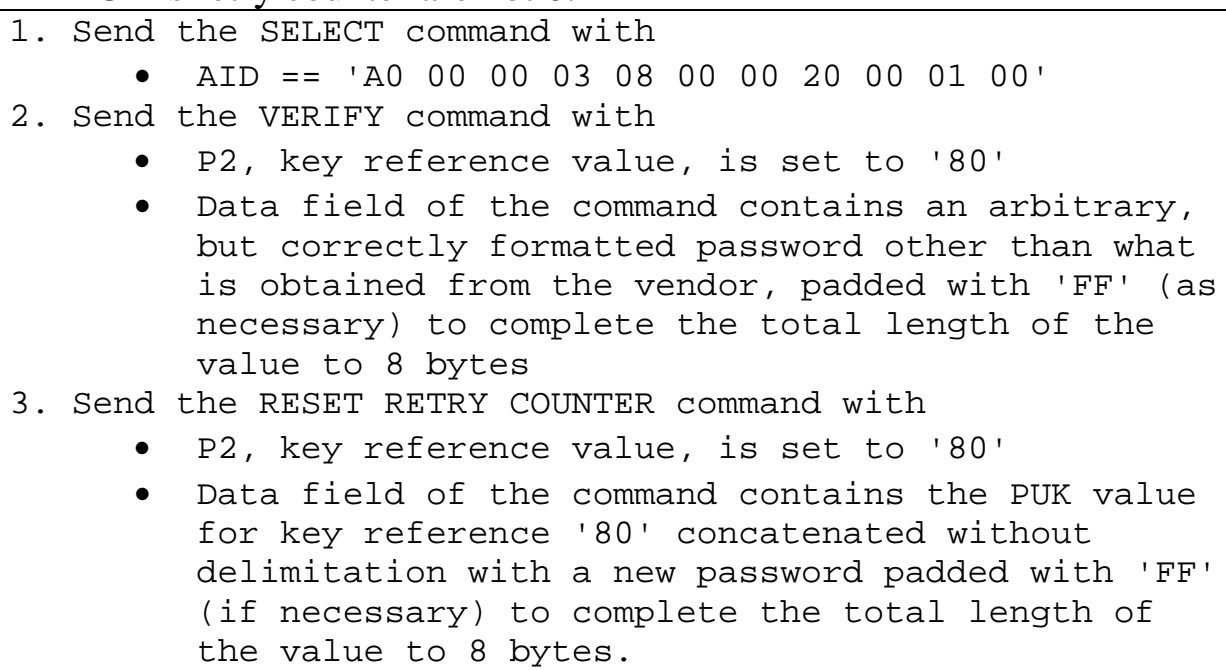 \\
\hline
\end{tabular}




\begin{tabular}{|c|c|}
\hline & $\begin{array}{l}\text { 4. Obtain number of remaining retries of the ' } 80 \text { ' key } \\
\text { reference by sending the VERIFY command with } \\
\text { - P2, key reference value, is set to ' } 80^{\prime} \\
\text { - P1 parameter is ' } \odot \odot \text { ' and both } L_{c} \text { and the data } \\
\text { field are absent } \\
\text { 5. Send the VERIFY command with } \\
\text { - P2, key reference value, is set to ' } 80^{\prime} \\
\text { - Data field of the command contains the new } \\
\text { password value, padded with 'FF' (if necessary) } \\
\text { to complete the total length of the value to } 8 \\
\text { bytes }\end{array}$ \\
\hline Expected Result(s) & $\begin{array}{l}\text { 1. From Step } 1 \text {, the command returns the application property template } \\
\text { with the status word ' } 9000 \text { '. } \\
\text { 2. From Step 2, the command returns status word ' } 63 \mathrm{CX} \text { ' (where ' } \mathrm{X} \text { ' is } \\
\text { the number of retries remaining). The retry counter is decremented } \\
\text { by } 1 \text {. } \\
\text { 3. From Step 3, the command returns status word ' } 9000 \text { '. } \\
\text { 4. From Step 4, the command returns status word ' } 63 \mathrm{CX} \text { ' (where ' } \mathrm{X} \text { ' is } \\
\text { the number of retries remaining). Verify that ' } \mathrm{X} \text { ' from this step is } \\
\text { greater than ' } \mathrm{X} \text { ' from Step } 2 \text { and is equal to the reset retry value. } \\
\text { 5. From Step 5, the command returns status word ' } 9000 \text { '. }\end{array}$ \\
\hline Postcondition(s) & The Derived PIV Application Password has been changed. \\
\hline
\end{tabular}

\subsubsection{Reset Retry Counter with an Invalid Key Reference}

\begin{tabular}{|c|c|}
\hline Test Assertion & TA-08.03.06.02 \\
\hline Purpose & $\begin{array}{l}\text { Verifies that the Derived PIV Application responds appropriately to the } \\
\text { RESET RETRY COUNTER command for an invalid key reference. }\end{array}$ \\
\hline DTR(s) & $\begin{array}{ll} & \text { DTR-06.02.02.09 } \\
- & \text { DTR-06.03.01.01 } \\
- & \text { DTR-06.03.07.01 } \\
\end{array}$ \\
\hline $\begin{array}{l}\text { Vendor } \\
\text { Documentation }\end{array}$ & None. \\
\hline Precondition(s) & $\begin{array}{l}\text { - A token with the Derived PIV Application is inserted into an } \\
\text { appropriate token reader. } \\
\text { - Suitable drivers have been loaded between the test system and an } \\
\text { instance of the reader. } \\
\text { - The PUK is recorded. }\end{array}$ \\
\hline Test Scenario & $\begin{array}{l}\text { 1. Send the SELECT command with } \\
\qquad \text { AID }==\text { 'A } \odot \odot \odot \odot \odot 3 \odot 8 \odot \odot \odot \odot 2 \odot \odot \odot \odot 1 \odot \odot \text { ' } \\
\text { 2. Send the RESET RETRY COUNTER command with } \\
\text { - P2, key reference value, is set to a value other } \\
\text { than ' } 8 \odot \text { ' } \\
\text { - Data field of the command contains the PUK value } \\
\text { for key reference ' } 8 \odot \text { ', concatenated without } \\
\text { delimitation with a valid new password padded }\end{array}$ \\
\hline
\end{tabular}




\begin{tabular}{|l|l|}
\hline & $\begin{array}{r}\text { with 'FF' (if necessary) to complete the total } \\
\text { length of the value to } 8 \text { bytes }\end{array}$ \\
\hline Expected Result(s) & $\begin{array}{l}\text { 1. From Step 1, the command returns the application property template } \\
\text { with the status word '90 00'. } \\
\text { 2. From Step 2, the command returns an error status word and the } \\
\text { PUK's retry counter remains unchanged. }\end{array}$ \\
\hline Postcondition(s) & Current password is unchanged. \\
\hline
\end{tabular}

\subsubsection{Reset Retry Counter with an Incorrect Length and Padding for the New Password}

\begin{tabular}{|c|c|}
\hline Test Assertion & TA-08.03.06.03 \\
\hline Purpose & $\begin{array}{l}\text { Verifies that the Derived PIV Application does not set a new password } \\
\text { with the RESET RETRY COUNTER command when the password } \\
\text { length and padding requirements are not met. }\end{array}$ \\
\hline $\operatorname{DTR}(\mathrm{s})$ & $\begin{array}{ll}- & \text { DTR-06.02.02.09 } \\
\text { - } & \text { DTR-06.03.01.01 } \\
\text { - } & \text { DTR-06.03.07.04 } \\
\end{array}$ \\
\hline $\begin{array}{l}\text { Vendor } \\
\text { Documentation }\end{array}$ & None. \\
\hline Precondition(s) & $\begin{array}{l}\text { - A token with the Derived PIV Application is inserted into an } \\
\text { appropriate token reader. } \\
\text { - Suitable drivers have been loaded between the test system and an } \\
\text { instance of the reader. } \\
\text { - The Derived PIV Application Password and the PUK are recorded. } \\
\text { - The Derived PIV Application Password's retry counter and the } \\
\text { PUK's retry counter are not 0. }\end{array}$ \\
\hline Test Scenario & 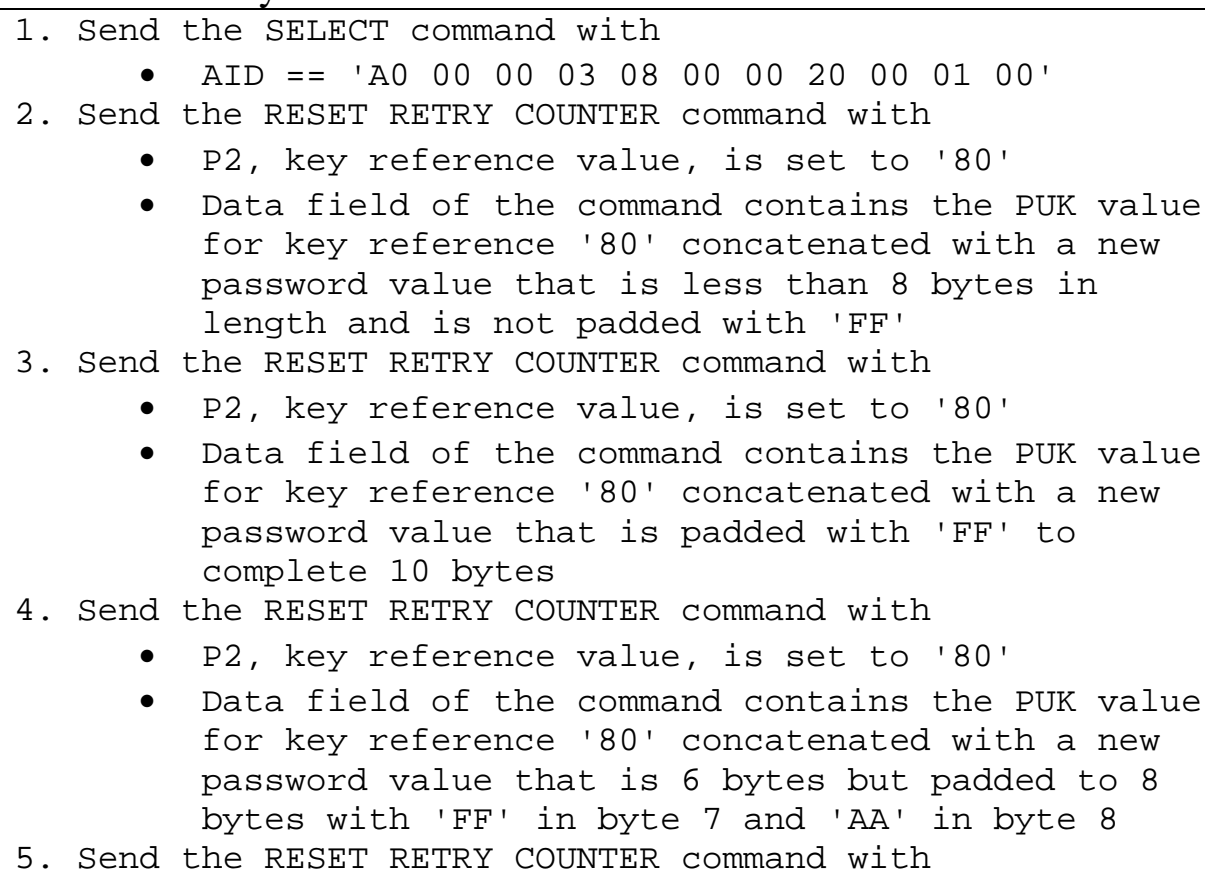 \\
\hline
\end{tabular}




\begin{tabular}{|c|c|}
\hline & $\begin{array}{l}\text { - P2, key reference value, is set to ' } 80^{\prime} \\
\text { - Data field of the command contains the PUK value } \\
\text { for key reference '80' concatenated with a new } \\
\text { password value that is less than } 6 \text { bytes padded } \\
\text { with 'FF' to complete } 8 \text { bytes } \\
\text { 6. Send the VERIFY command with } \\
\text { - P2, key reference value, is set to ' } 80 \text { ' } \\
\text { - Data field of the command will contain the } \\
\text { correct Derived PIV Application Password, padded } \\
\text { with 'FF' (if necessary) to complete the total } \\
\text { length of the value to } 8 \text { bytes }\end{array}$ \\
\hline Expected Result(s) & $\begin{array}{l}\text { 1. From Step 1, the command returns the application property template } \\
\text { with the status word ' } 90 \text { 00'. } \\
\text { 2. From Step 2, the command returns status word '6A 80' (incorrect } \\
\text { parameter in command data field). } \\
\text { 3. From Step 3, the command returns status word '6A 80' (incorrect } \\
\text { parameter in command data field). } \\
\text { 4. From Step 4, the command returns status word '6A 80' (incorrect } \\
\text { parameter in command data field). } \\
\text { 5. From Step 5, the command returns status word '6A 80' (incorrect } \\
\text { parameter in command data field). } \\
\text { 6. From Step 6, the command returns status word '90 00'. }\end{array}$ \\
\hline Postcondition(s) & Current password is unchanged. \\
\hline
\end{tabular}

\subsubsection{Reset Retry Counter with an Incorrect Format for the New Password}

\begin{tabular}{|c|c|}
\hline Test Assertion & TA-08.03.06.04 \\
\hline Purpose & $\begin{array}{l}\text { Verifies that the Derived PIV Application does not set a new password } \\
\text { with the RESET RETRY COUNTER command when the format } \\
\text { requirements of the new password are not met. }\end{array}$ \\
\hline DTR(s) & $\begin{array}{ll}\text { - } & \text { DTR-06.02.02.09 } \\
\text { - } & \text { DTR-06.03.01.01 } \\
\text { - } & \text { DTR-06.03.07.04 } \\
\text { - } & \text { DTR-06.03.07.05 } \\
\text { - } & \text { DTR-06.03.07.06 } \\
\text { - } & \text { DTR-06.03.07.07 }\end{array}$ \\
\hline $\begin{array}{l}\text { Vendor } \\
\text { Documentation }\end{array}$ & None. \\
\hline Precondition(s) & $\begin{array}{l}\text { - A token with the Derived PIV Application is inserted into an } \\
\text { appropriate token reader. } \\
\text { - Suitable drivers have been loaded between the test system and an } \\
\text { instance of the reader. } \\
\text { - The Derived PIV Application Password and the PUK are recorded. } \\
\text { - The Derived PIV Application Password's retry counter and the } \\
\text { PUK's retry counter are not } 0 .\end{array}$ \\
\hline
\end{tabular}




\begin{tabular}{|c|c|}
\hline Test Scenario & 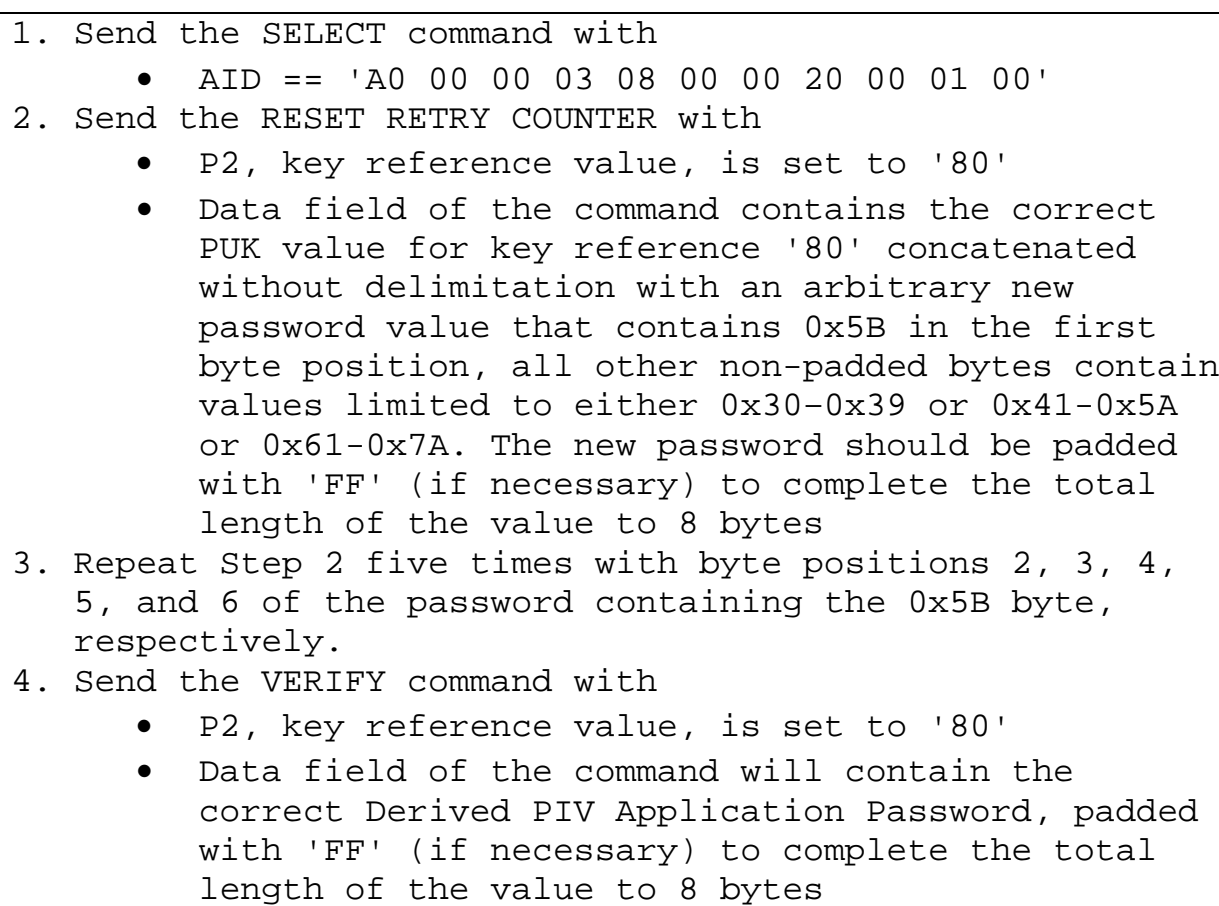 \\
\hline Expected Result(s) & $\begin{array}{l}\text { 1. From Step 1, the command returns the application property template } \\
\text { with the status word ' } 9000 \text { '. } \\
\text { 2. From Step 2, the command returns status word '6A 80' (incorrect } \\
\text { parameter in command data field). } \\
\text { 3. From Step 3, each time the command returns status word '6A 80' } \\
\text { (incorrect parameter in command data field). } \\
\text { 4. From Step 4, the command returns status word '90 00'. }\end{array}$ \\
\hline Postcondition(s) & Current password is unchanged. \\
\hline
\end{tabular}

\subsubsection{Reset Retry Counter with an incorrect length for the PUK}

\begin{tabular}{|c|c|}
\hline Test Assertion & TA-08.03.06.05 \\
\hline Purpose & $\begin{array}{l}\text { Verifies that the Derived PIV Application does not set a new password } \\
\text { with the RESET RETRY COUNTER command when the PUK length is } \\
\text { incorrect. }\end{array}$ \\
\hline DTR(s) & $\begin{array}{ll} & \text { DTR-06.02.02.09 } \\
\text { - } & \text { DTR-06.03.01.01 } \\
\text { - } & \text { DTR-06.03.07.04 } \\
\text { - } & \text { DTR-06.03.07.05 } \\
\text { - } & \text { DTR-06.03.07.06 } \\
\text { - } & \text { DTR-06.03.07.07 }\end{array}$ \\
\hline $\begin{array}{l}\text { Vendor } \\
\text { Documentation }\end{array}$ & None. \\
\hline Precondition(s) & $\begin{array}{l}\text { - A token with the Derived PIV Application is inserted into an } \\
\text { appropriate token reader. }\end{array}$ \\
\hline
\end{tabular}




\begin{tabular}{|c|c|}
\hline & $\begin{array}{l}\text { - Suitable drivers have been loaded between the test system and an } \\
\text { instance of the reader. } \\
\text { - The Derived PIV Application Password and the PUK are recorded. } \\
\text { - The Derived PIV Application Password's retry counter is not } 0 \text { and } \\
\text { the PUK's retry counter is greater than } 1 \text {. }\end{array}$ \\
\hline Test Scenario & 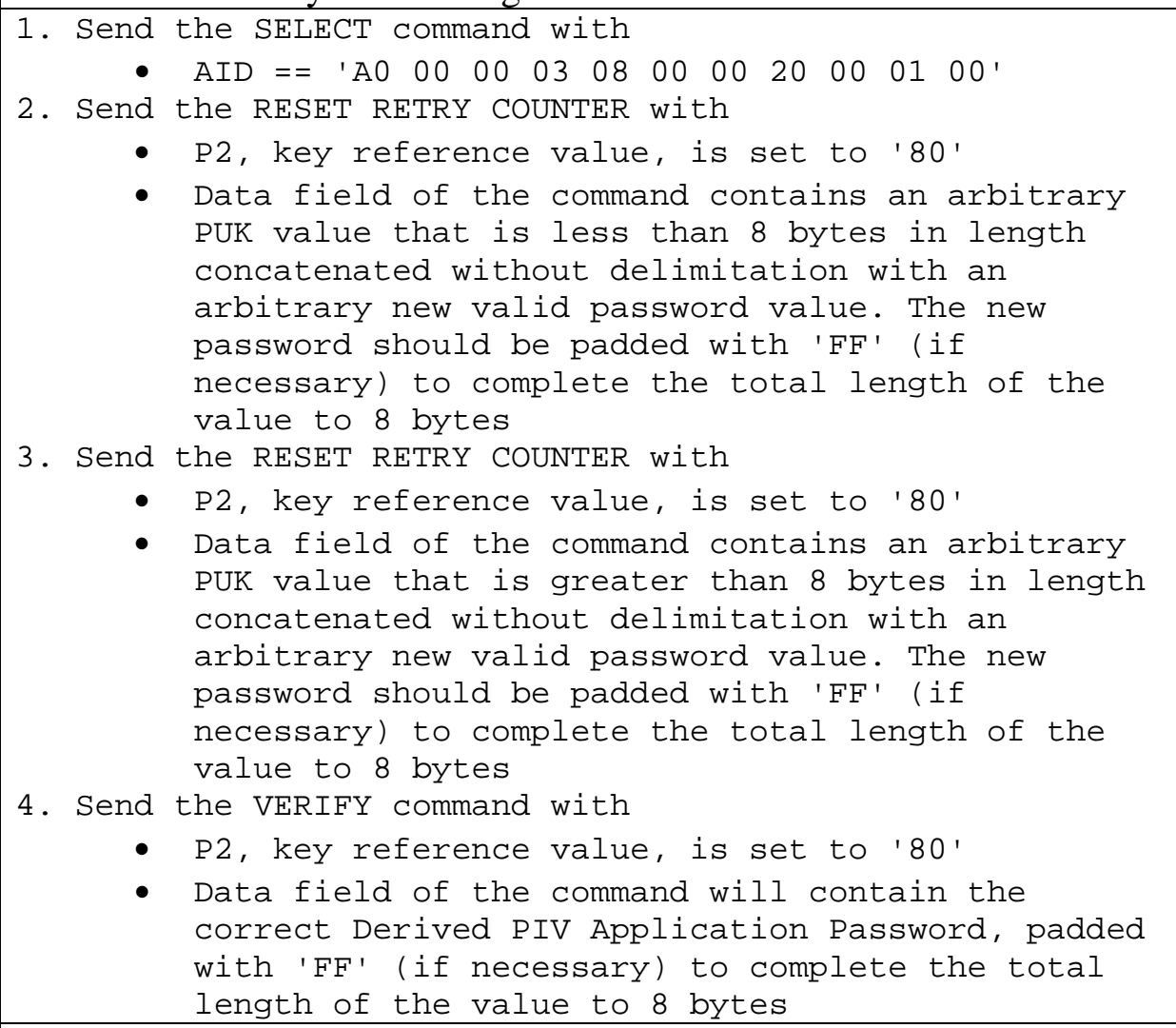 \\
\hline Expected Result(s) & $\begin{array}{l}\text { 1. From Step 1, the command returns the application property template } \\
\text { with the status word ' } 90 \text { 00'. } \\
\text { 2. From Step 2, the command returns either status word: } 1 \text { ) ' } 6 \mathrm{~A} 80 \text { ' } \\
\text { (incorrect parameter in command data field) or } 2 \text { ) '63 CX' and the } \\
\text { PUK's retry counter is decremented by } 1 \text { (where ' } \mathrm{X} \text { ' is the number of } \\
\text { tries remaining). } \\
\text { 3. From Step 3, the command returns either status word: } 1 \text { ) ' } 6 \mathrm{~A} 80 \text { ' } \\
\text { (incorrect parameter in command data field) or } 2 \text { ) '63 CX' and the } \\
\text { PUK's retry counter is decremented by } 1 \text { (where ' } \mathrm{X} \text { ' is the number of } \\
\text { tries remaining). } \\
\text { 4. From Step 4, the command returns status word ' } 9000 \text { '. }\end{array}$ \\
\hline Postcondition(s) & Current password is unchanged. \\
\hline
\end{tabular}

\subsubsection{Reset Retry Counter using an Incorrect PUK}




\begin{tabular}{|c|c|}
\hline Purpose & $\begin{array}{l}\text { Verifies that the Derived PIV Application does not change the value of } \\
\text { the Derived PIV Application Password when an incorrect PUK is } \\
\text { provided. }\end{array}$ \\
\hline DTR(s) & $\begin{array}{l}\text { - } \mathrm{DTR}-06.02 .02 .09 \\
\text { - } \mathrm{DTR}-06.03 .01 .01 \\
\text { - } \mathrm{DTR}-06.03 .07 .02 \\
\text { - } \mathrm{DTR}-06.03 .07 .03 \\
\text { - } \mathrm{DTR}-06.03 .07 .05 \\
\text { - } \mathrm{DTR}-06.03 .07 .06 \\
\text { - } \mathrm{DTR}-06.03 .07 .07\end{array}$ \\
\hline $\begin{array}{l}\text { Vendor } \\
\text { Documentation }\end{array}$ & None. \\
\hline Precondition(s) & $\begin{array}{l}\text { - A token with the Derived PIV Application is inserted into an } \\
\text { appropriate token reader. } \\
\text { - Suitable drivers have been loaded between the test system and an } \\
\text { instance of the reader. } \\
\text { - The Derived PIV Application Password and the PUK are recorded. } \\
\text { - The Derived PIV Application Password's retry counter is not } 0 \text { and } \\
\text { the PUK's retry counter greater than } 1 \text {. }\end{array}$ \\
\hline Test Scenario & 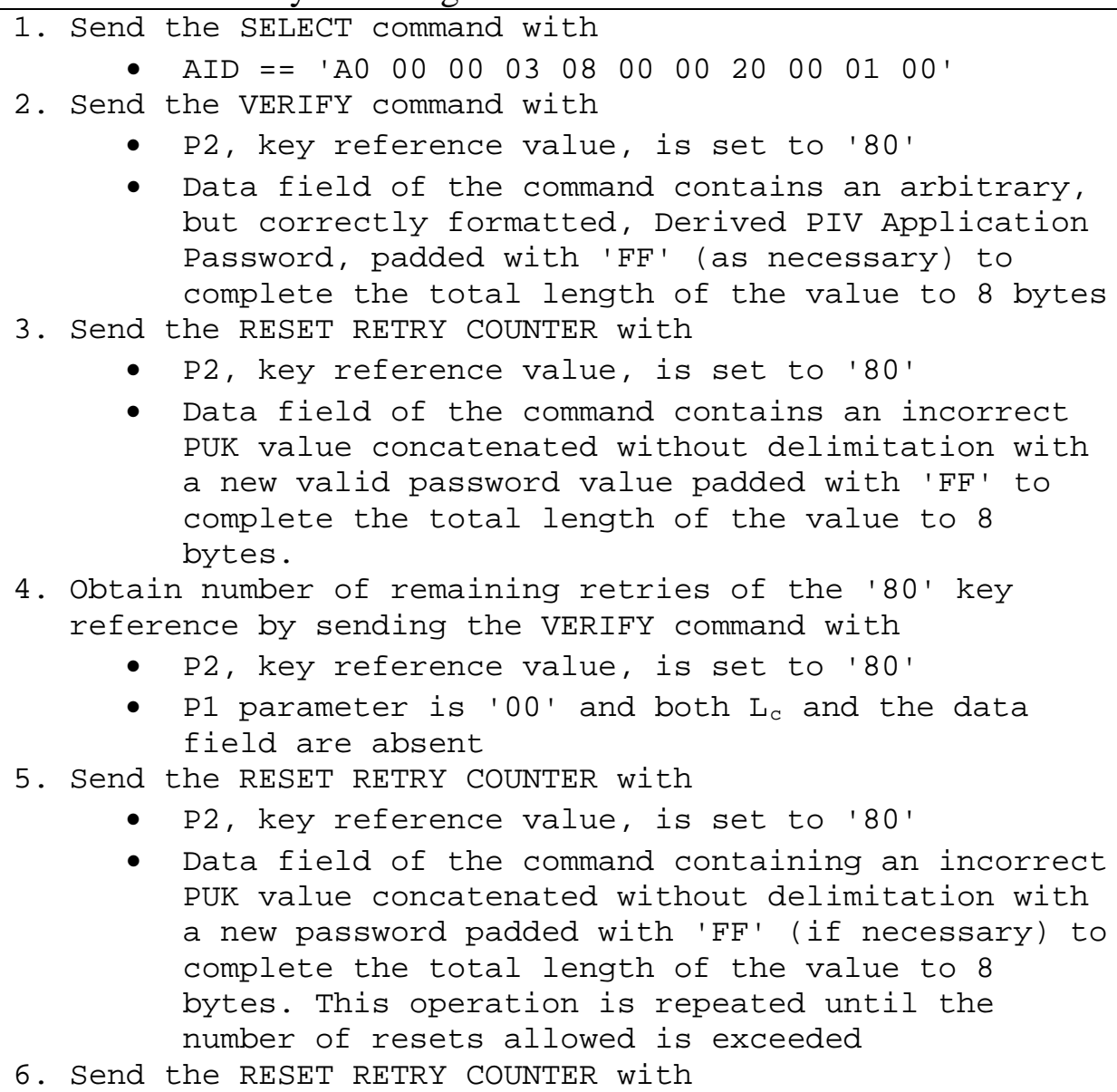 \\
\hline
\end{tabular}




\begin{tabular}{|c|c|}
\hline & $\begin{array}{l}\text { - P2, key reference value, is set to ' } 80 \text { ' } \\
\text { - Data field of the command contains the correct } \\
\text { PUK value concatenated without delimitation with } \\
\text { a new correctly-formatted password value padded } \\
\text { to } 8 \text { bytes with 'FF' (if necessary) }\end{array}$ \\
\hline Expected Result(s) & $\begin{array}{l}\text { 1. From Step 1, the command returns the application property template } \\
\text { with the status word ' } 90 \text { 00'. } \\
\text { 2. From Step 2, the command returns status word ' } 63 \mathrm{CX} \text { ' (X == } \\
\text { number of retries left for the Derived PIV Application Password) } \\
\text { 3. From Step 3, the command returns status word '63 CX' (where 'X' } \\
\text { indicates the number of further allowed retries for the PUK) } \\
\text { 4. From Step 4, the command returns status word '63 CX' (where 'X' is } \\
\text { the number of retries remaining for the Derived PIV Application } \\
\text { Password. Verify that 'X' from this step is the same as 'X' from Step } \\
\text { 2. } \\
\text { 5. From Step 5, the command returns status word '63 CX' (where 'X' } \\
\text { indicates the number of further allowed retries for the PUK) and the } \\
\text { PUK's retry counter is decremented each time. The command } \\
\text { returns status word ' } 69 \text { 83' (reset operation blocked) when the } \\
\text { command is invoked after the value of 'X' becomes } 0 \text {. } \\
\text { 6. From Step 6, the command returns status word '69 83' (reset } \\
\text { operation blocked). }\end{array}$ \\
\hline Postcondition(s) & The Reset Retry Counter command is blocked. \\
\hline
\end{tabular}

\subsubsection{PUT DATA Command}

\subsubsection{Put Data for various Data Objects of the Derived PV Application}

\begin{tabular}{|c|c|}
\hline Test Assertion & TA-08.03.07.01 \\
\hline Purpose & $\begin{array}{l}\text { Verifies that the Derived PIV Application responds appropriately to the } \\
\text { PUT DATA command. }\end{array}$ \\
\hline DTR(s) & $\begin{array}{ll}\text { - } & \text { DTR-06.02.02.02 } \\
\text { - } & \text { DTR-06.02.02.03 } \\
\text { - } & \text { DTR-06.02.02.04 } \\
\text { - } & \text { DTR-06.02.02.05 } \\
\text { - } & \text { DTR-06.02.02.06 } \\
\text { - } & \text { DTR-06.02.02.07 } \\
\text { - } & \text { DTR-06.02.02.08 } \\
\text { - } & \text { DTR-06.02.02.09 } \\
\text { - } & \text { DTR-06.03.01.01 } \\
\text { - } & \text { DTR-06.03.08.01 }\end{array}$ \\
\hline $\begin{array}{l}\text { Vendor } \\
\text { Documentation }\end{array}$ & None. \\
\hline
\end{tabular}




\begin{tabular}{|c|c|}
\hline Precondition(s) & $\begin{array}{l}\text { - A token with the Derived PIV Application is inserted into an } \\
\text { appropriate token reader. } \\
\text { - Suitable drivers have been loaded between the test system and an } \\
\text { instance of the reader. } \\
\text { - The Derived PIV Application Token Management Key is recorded. } \\
\text { - The mutual authentication of the Derived PIV Application and the } \\
\text { Test Toolkit Application has not been performed. } \\
\text { - Data objects to be loaded are equal to the minimum container sizes } \\
\text { specified for that object. }{ }^{14}\end{array}$ \\
\hline Test Scenario & 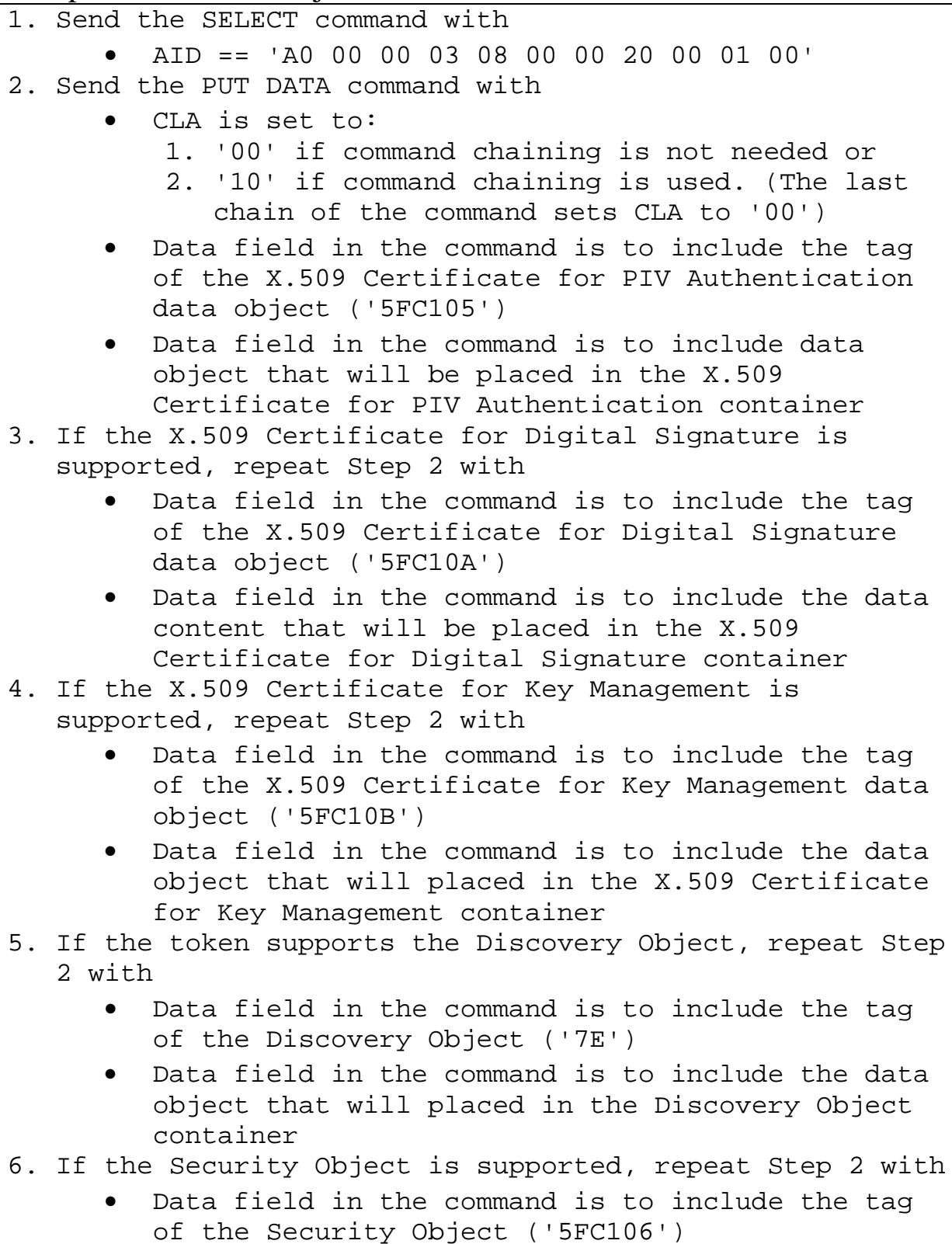 \\
\hline
\end{tabular}

${ }^{14}$ Data objects for the containers do not have to be properly formatted for this test. 


\begin{tabular}{|c|c|}
\hline & 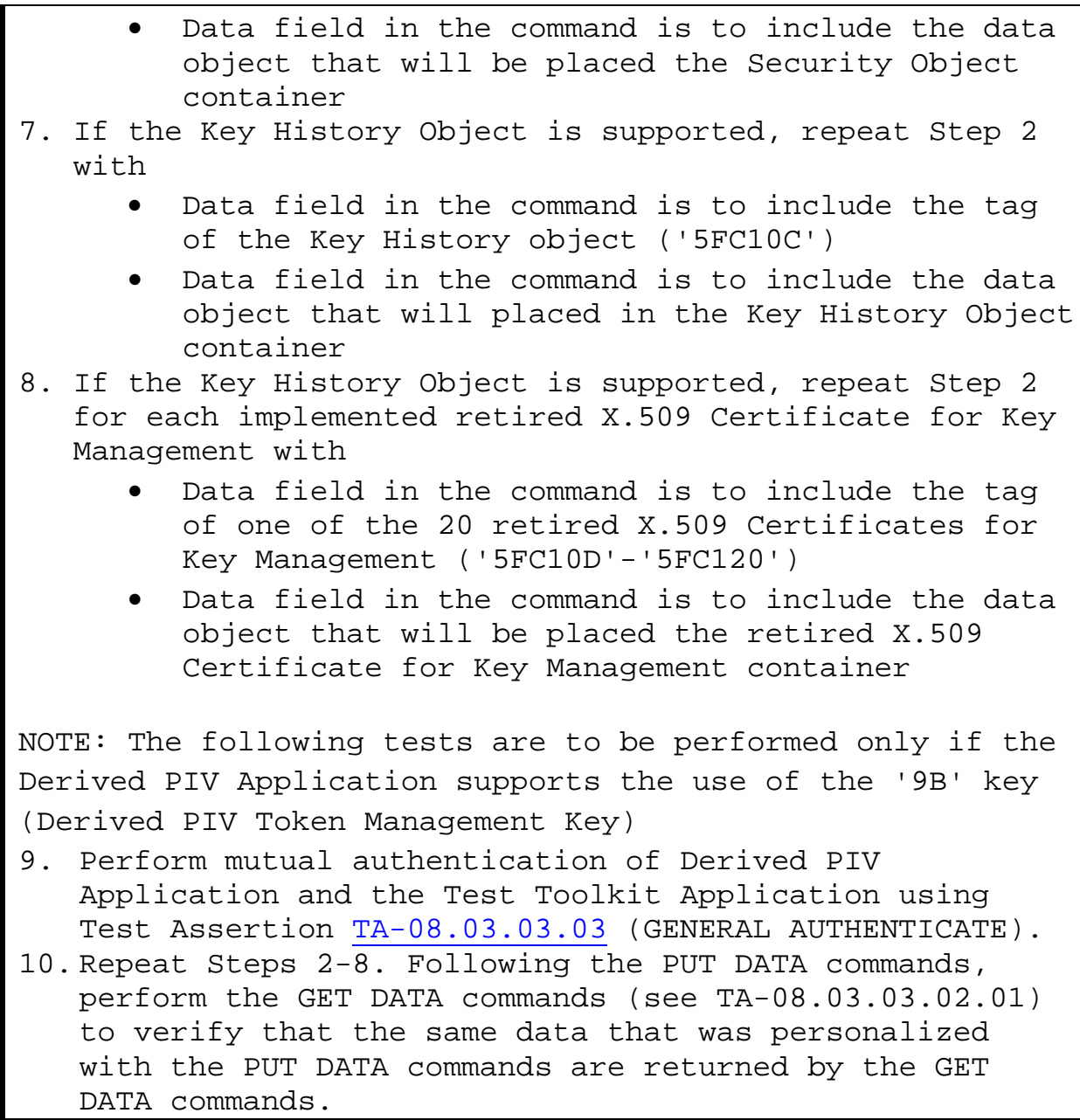 \\
\hline Expected Result(s) & $\begin{array}{l}\text { 1. From Step 1, the command returns the application property template } \\
\text { with the status word ' } 90 \text { 00'. } \\
\text { 2. From Step } 2 \text { through Step 8, the commands return status word '69 } \\
82 \text { ' (security status not satisfied). } \\
\text { 3. From Step 9, the test results are consistent with those expected as } \\
\text { part of TA-08.03.03.03: } \\
\text { a. From Step } 1 \text { of TA-08.03.03.03, the command returns the } \\
\text { application property template with the status word '90 00'. } \\
\text { b. From Step } 2 \text { of TA-08.03.03.03, the command returns with } \\
\text { the witness followed by status word ' } 90 \text { 00'. } \\
\text { c. From Step } 3 \text { of TA-08.03.03.03, the Derived PIV } \\
\text { Application verifies the decrypted witness and then responds } \\
\text { with encryption of the challenge sent by Test Toolkit } \\
\text { Application followed by status word '90 00'. Decrypt the } \\
\text { encrypted challenge and compare it to the one sent to the } \\
\text { token. } \\
\text { 4. From Step 10, all commands return status word '90 00', and input } \\
\text { and output data match. }\end{array}$ \\
\hline Postcondition(s) & N/A \\
\hline
\end{tabular}




\subsubsection{GENERATE ASYMMETRIC KEY PAIR Command}

\subsubsection{Generate Asymmetric Key Pair for the Various Keys}

\begin{tabular}{|c|c|}
\hline Test Assertion & TA-08.03.08.01 \\
\hline Purpose & $\begin{array}{l}\text { Verifies that the Derived PIV Application responds appropriately to the } \\
\text { GENERATE ASYMMETRIC KEY PAIR command. }\end{array}$ \\
\hline DTR(s) & $\begin{array}{ll}\text { - } & \text { DTR-06.02.02.09 } \\
\text { - } & \text { DTR-06.03.01.01 } \\
\text { - } & \text { DTR-06.03.04.05 } \\
\text { - } & \text { DTR-06.03.09.01 } \\
\text { - } & \text { DTR-06.03.09.02 } \\
\end{array}$ \\
\hline $\begin{array}{l}\text { Vendor } \\
\text { Documentation }\end{array}$ & $\begin{array}{l}\text { The vendor to provide documentation specifying the cryptographic } \\
\text { mechanism identifiers (from Table } 5 \text { of [SP800-73], Part 1) that have } \\
\text { been implemented. }\end{array}$ \\
\hline Precondition(s) & $\begin{array}{l}\text { - A token with the Derived PIV Application is inserted into an } \\
\text { appropriate token reader. } \\
\text { - Suitable drivers have been loaded between the test system and an } \\
\text { instance of the reader. } \\
\text { - The Derived PIV Application Token Management Key is recorded. } \\
\text { - The mutual authentication of the Derived PIV Application and the } \\
\text { Test Toolkit Application has not been performed. } \\
\text { - The Derived PIV Application Password is recorded. } \\
\text { - The Derived PIV Application Password's retry counter is not } 0 \text {. }\end{array}$ \\
\hline Test Scenario & 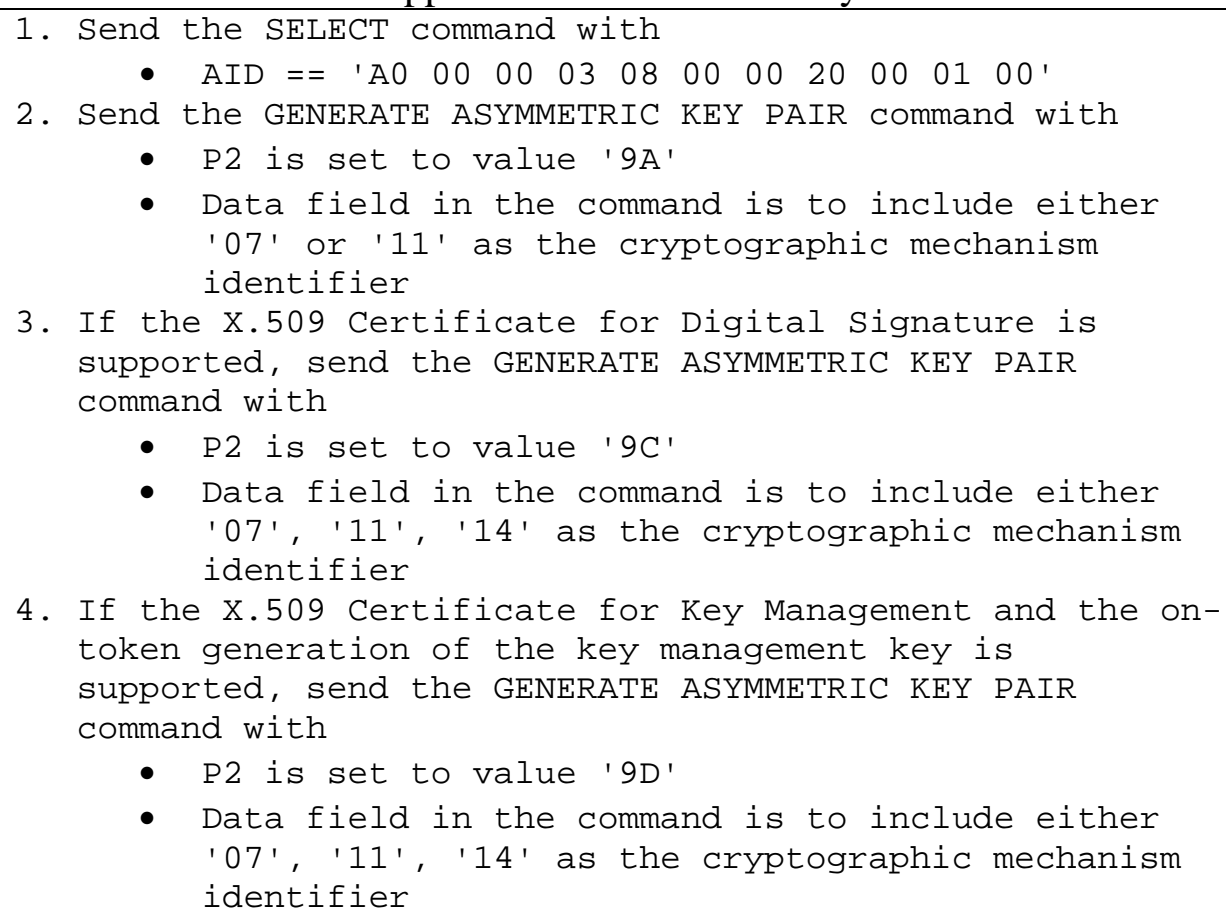 \\
\hline
\end{tabular}


NOTE: The following tests are to be performed only if the Derived PIV Application supports the use of the '9B' key (Derived PIV Token Management Key)

5. Perform mutual authentication of Derived PIV Application and the Test Toolkit Application using Test Assertion TA-08.03.03.03 (GENERAL AUTHENTICATE)

6. Repeat Steps 2, 3, and 4 .

7. Repeat Step 2 with the cryptographic mechanism identifier value in the data field set to a value that is not supported by the Derived PIV Application.

8. Repeat Step 2 with P2 set to a key reference value that is not supported by the Derived PIV Application.

9. Send the VERIFY command with

- P2, key reference value, is set to '80'

- Data field of the command will contain the correct Derived PIV Application Password, padded with 'FF' (if necessary) to complete the total length of the value to 8 bytes

10. Send the GENERAL AUTHENTICATE command

- CLA is set to:

1. ' $\odot \odot$ ' if command chaining is not needed or

2. ' 10 ' if command chaining is used. (The last chain of the command sets CLA to ' $\odot \odot^{\prime}$ ')

- P1, algorithm reference, is set to '07' or '11'

- P2, key reference, is set to '9A'

- Data field in the command is to include '81' specifying a challenge, followed by a randomly generated challenge, and ' $820{ }^{\circ}$ ' in order to request a response

11. If the X.509 Certificate for Digital Signature is supported, send the GENERAL AUTHENTICATE command

- CLA is set to:

1. ' $\odot \odot$ ' if command chaining is not needed or

2. '10' if command chaining is used. (The last chain of the command sets CLA to 'O०')

- $\mathrm{P} 1$, algorithm reference, is set to ' 07 ', '11' or '14'

- P2, key reference, is set to ' $9 C^{\prime}$

- Data field in the command is to include '81' specifying a challenge, followed by a randomly generated challenge, and ' $820{ }^{\circ}$ ' in order to request a response

12. If the X.509 Certificate for Key Management and the on-token generation of the key management key is supported, send the GENERAL AUTHENTICATE command

- CLA is set to:

1. ' $\odot \odot$ ' if command chaining is not needed or

2. ' 10 ' if command chaining is used. (The last chain of the command sets CLA to 'O०')

- $\mathrm{P} 1$, algorithm reference, is set to ' 07 ', ' $11^{\prime}$ or '14'

- P2, key reference, is set to '9D' indicating the key management key 


\begin{tabular}{|c|c|}
\hline & $\begin{array}{l}\text { - Data field in the command is to include one of } \\
\text { the following: } \\
\text { 1. If } \mathrm{P} 1={ }^{\prime} 07 \text { ', the template ' } 81 \text { ' contains a } \\
\text { key encrypted using the key management public } \\
\text { key returned in step } 6 \text {. } \\
\text { 2. If } 1=\text { ' } 11 \text { ' or ' } 14 \text { ', the template ' } 85 \text { ' } \\
\text { contain the other party's public key. }{ }^{15}\end{array}$ \\
\hline Expected Result(s) & $\begin{array}{l}\text { 1. From Step 1, the command returns the application property template } \\
\text { with the status word ' } 90 \text { 00'. } \\
\text { 2. From Step 2, 3, and 4, the command returns status word '69 82' } \\
\text { (security status not satisfied). } \\
\text { 3. From Step 5, the test results are consistent with those expected as } \\
\text { part of TA-08.03.03.03: } \\
\text { a. From Step } 1 \text { of TA-08.03.03.03, the command returns the } \\
\text { application property template with the status word '90 00'. } \\
\text { b. From Step } 2 \text { of TA-08.03.03.03, the command returns with } \\
\text { the witness followed by status word '90 00'. } \\
\text { c. From Step } 3 \text { of TA-08.03.03.03, the Derived PIV } \\
\text { Application verifies the decrypted witness and then responds } \\
\quad \text { with encryption of the challenge sent by Test Toolkit } \\
\quad \text { Application followed by status word ' } 90 \text { 00'. Decrypt the } \\
\text { encrypted challenge and compare it to the one sent to the } \\
\text { token. } \\
\text { 4. From Step 6, the commands return status word '90 00' and the data } \\
\text { field contains the '7F49' template with the generated public key, } \\
\text { which consists of either a modulus and public exponent (RSA) or a } \\
\text { point (elliptic curve cryptography). } \\
\text { 5. From Step 7, the command returns status word '6A 80' (incorrect } \\
\text { parameter command data field). } \\
\text { 6. From Step 8, the command returns status word '6A 86' (incorrect } \\
\text { parameter P2). } \\
\text { 7. From Step 9, the command returns status word '90 00'. } \\
\text { 8. From Step 10, the command returns the signed challenge with status } \\
\text { word '90 00'. Verify the signed challenge using the Derived PIV } \\
\text { Authentication public key that was returned in Step 6. } \\
\text { 9. From Step 11, the command returns the signed challenge with status } \\
\text { word '90 00'. Verify the signed challenge using the digital signature } \\
\text { public key that was returned in Step 6. } \\
\text { received in the response from the token. For algorithm reference '11' } \\
\text { or '14' as P1 value, the command returns the shared secret Z } 16 \text { with }\end{array}$ \\
\hline
\end{tabular}

\footnotetext{
${ }^{15}$ Template '85' contains the other party's public key, a point on Curve P-256 or P-384, encoded as '04' || X || Y, without the use of point compression, as described in Section 2.3.3 of [SEC1].

${ }^{16} \mathrm{Z}$ is the $\mathrm{X}$ coordinate of point $\mathrm{P}$ as defined in [SP800-56A], Section 5.7.1.2
} 


\begin{tabular}{|l|l|}
\hline & $\begin{array}{c}\text { status word '90 00'. Compare the shared secret computed by the } \\
\text { token with the shared secret computed off token (using the key } \\
\text { management public key that was returned in Step 6). }\end{array}$ \\
\hline Postcondition(s) & The token has newly generated private key(s). \\
\hline
\end{tabular}




\section{Test Assertions for the Derived PIV Application Data Model}

This section lists the test assertions used to determine conformity to the derived test requirements (DTR) listed in Section 7. The Implementation Under Test (IUT), the Derived PIV Data Objects loaded on a Derived PIV Application by an issuer, must meet the stated objective(s) of the assertion by way of a test or submission of artifacts in order to be deemed conformant to the associated DTR(s).

\subsection{BER-TLV Conformance}

The following assumptions apply to the test assertions within this section.

\begin{tabular}{|c|c|}
\hline 1 & $\begin{array}{l}\text { When the length of the value field is between } 0 \text { and } 127 \text { bytes, the length field should } \\
\text { consist of a single byte where bit } 8 \text { is set to } 0 \text { and bits } 7 \text { to } 1 \text { encode the number of } \\
\text { bytes in the value field. } \\
\text { When the length of the value field is greater than } 127 \text { bytes, the length field consists } \\
\text { of two or more bytes. }{ }^{17} \text { The first byte is ' } 81 \text { ', ' } 82 \text { ', ' } 83 \text { ' or ' } 84 \text { ' where the low order } \\
\text { nibble of each of these possible first-byte values (1, } 2,3 \text {, or } 4 \text { respectively) encodes } \\
\text { the number of subsequent bytes in the length field. These subsequent bytes are taken } \\
\text { together in order to be a big-endian integer encoding the number of bytes in the value } \\
\text { field. Table } 1 \text { shows the encoding of the length field. }\end{array}$ \\
\hline 2 & Except for the Discovery Object tag, each BER-TLV tag is encoded as three bytes. \\
\hline 3 & Each data object returned is appended with a 2 byte status word. \\
\hline 4 & $\begin{array}{l}\text { All variable length value fields can have zero lengths, which will result in a tag length } \\
\text { field being immediately followed by the next tag, if applicable. }\end{array}$ \\
\hline 5 & $\begin{array}{l}\text { The final byte of the command string can be set to } 0 x 00 \text { to retrieve an entire data } \\
\text { object regardless of the size of that object. }\end{array}$ \\
\hline
\end{tabular}

\begin{tabular}{|c|c|l|l|}
\hline $\begin{array}{c}\text { Number of bytes in the } \\
\text { length field }\end{array}$ & First byte & \multicolumn{1}{|c|}{ Subsequent bytes } & \multicolumn{1}{|c|}{$\begin{array}{c}\text { Length of } \\
\text { the value field }\end{array}$} \\
\hline 1 byte & '00' to '7F' & None & 0 to 127 \\
\hline 2 byte & '81' & '00' to 'FF' & 0 to 255 \\
\hline 3 byte & '82' & '0000' to 'FFFF' & 0 to 65535 \\
\hline 4 byte & ' 83 ' & '000000' to 'FFFFFF' & 0 to 16777215 \\
\hline 5 byte & '84' & '00000000' to 'FFFFFFFF' & 0 to 4294967295 \\
\hline
\end{tabular}

Table 1 - Encoding of Length Field

\footnotetext{
${ }^{17}$ Use of the shortest encoding format is preferred.
} 


\subsubsection{X.509 Certificate for Derived PIV Authentication}

\subsubsection{BER-TLV of X.509 Certificate for Derived PIV Authentication}

\begin{tabular}{|c|c|}
\hline Test Assertion & TA-09.01.01.01 \\
\hline Purpose & $\begin{array}{l}\text { Verifies that the X.509 Certificate for Derived PIV Authentication } \\
\text { conforms to the Derived PIV data model requirements. }\end{array}$ \\
\hline DTR(s) & - $\quad$ DTR-07.01.02.01 \\
\hline Issuer Documentation & None. \\
\hline Precondition(s) & $\begin{array}{l}\text { - A token with the Derived PIV Application is inserted into an } \\
\text { appropriate token reader. } \\
\text { - Suitable drivers have been loaded between the test system and } \\
\text { an instance of the reader. }\end{array}$ \\
\hline Test Scenario & 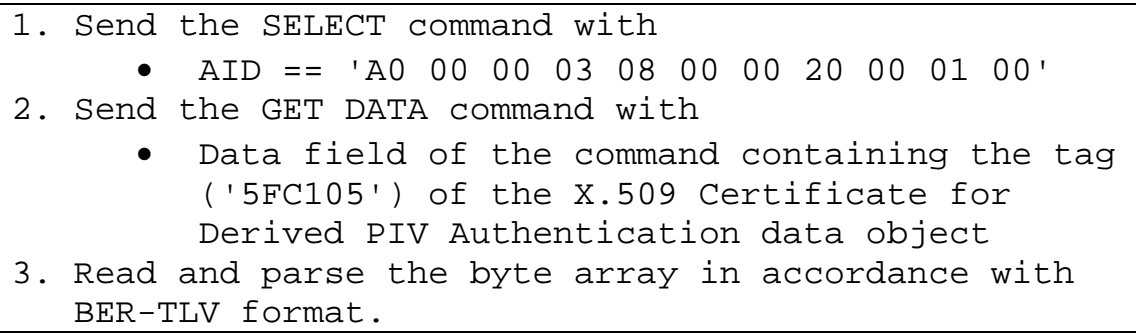 \\
\hline Expected Result(s) & $\begin{array}{l}\text { 1. From Step 1, the command returns the application property } \\
\text { template with the status word ' } 90 \text { 00'. } \\
\text { 2. From Step 2, the command returns status word ' } 90 \text { 00' along } \\
\text { with the X.509 Certificate for Derived PIV Authentication data } \\
\text { object. } \\
\text { 3. From Step 3, all mandatory tags for the X.509 Certificate for } \\
\text { Derived PIV Authentication data object are present in the order } \\
\text { indicated in Table } 10 \text { of [SP800-73], Part } 1 \text {. }\end{array}$ \\
\hline
\end{tabular}

\subsubsection{X.509 Certificate for Digital Signature}

\subsubsection{BER-TLV of X.509 Certificate for Digital Signature}

\begin{tabular}{|c|c|}
\hline Test Assertion & TA-09.01.02.01 \\
\hline Purpose & $\begin{array}{l}\text { Verifies that the X.509 Certificate for Digital Signature (if present) } \\
\text { conforms to the Derived PIV data model requirements. }\end{array}$ \\
\hline DTR(s) & $\begin{array}{ll}- & \text { DTR-07.01.01.01 } \\
\text { - } & \text { DTR-07.01.03.01 } \\
\end{array}$ \\
\hline Issuer Documentation & None. \\
\hline Precondition(s) & $\begin{array}{l}\text { - A token with the Derived PIV Application is inserted into an } \\
\text { appropriate token reader. } \\
\text { - Suitable drivers have been loaded between the test system and } \\
\text { an instance of the reader. }\end{array}$ \\
\hline
\end{tabular}




\begin{tabular}{|c|c|}
\hline Test Scenario & 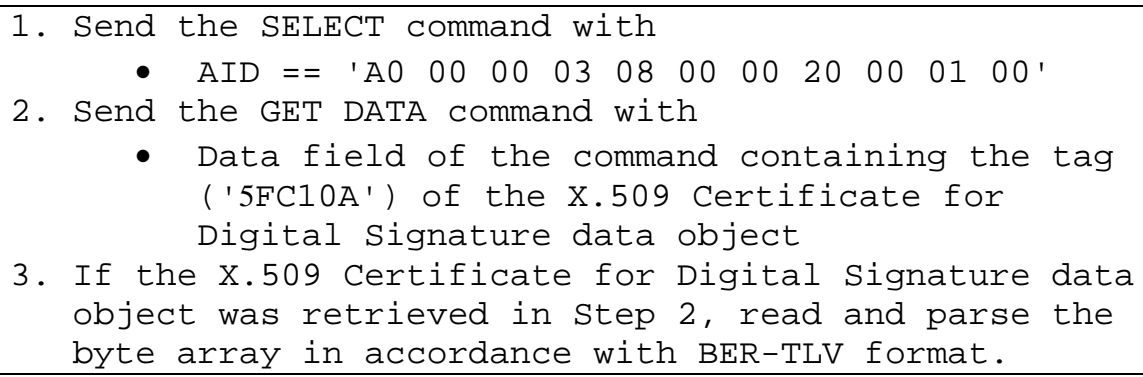 \\
\hline Expected Result(s) & $\begin{array}{l}\text { 1. From Step 1, the command returns the application property } \\
\text { template with the status word ' } 90 \text { 00'. } \\
\text { 2. From Step 2, the command returns one of the following: } \\
\text { - An X.509 Certificate for Digital Signature data object } \\
\text { followed by status word ' } 9000 \text { '; } \\
\text { - A zero-length data object followed by status word '90 00' - } \\
\text { which indicates that a container for the X.509 certificate } \\
\text { exists, but it has not been personalized; or } \\
\text { - Status word '6A } 82 \text { ' (data object not found) - which } \\
\text { indicates the container for the X.509 certificate does not } \\
\text { exist } \\
\text { 3. From Step 3, all mandatory tags for the X.509 Certificate for } \\
\text { Digital Signature data object are present in the order indicated } \\
\text { in Table } 15 \text { of [SP800-73], Part } 1 \text {. }\end{array}$ \\
\hline
\end{tabular}

\subsubsection{X.509 Certificate for Key Management}

\subsubsection{BER-TLV of X.509 Certificate for Key Management}

\begin{tabular}{|c|c|}
\hline Test Assertion & TA-09.01.03.01 \\
\hline Purpose & $\begin{array}{l}\text { Verifies that the X.509 Certificate for Key Management (if } \\
\text { present) conforms to the Derived PIV data model requirements. }\end{array}$ \\
\hline DTR(s) & $\begin{array}{ll}\text { - } & \text { DTR-07.01.01.01 } \\
\text { - } & \text { DTR-07.01.04.01 }\end{array}$ \\
\hline Issuer Documentation & None. \\
\hline Precondition(s) & $\begin{array}{l}\text { - A token with the Derived PIV Application is inserted into an } \\
\text { appropriate token reader. } \\
\text { - Suitable drivers have been loaded between the test system and } \\
\text { an instance of the reader. }\end{array}$ \\
\hline Test Scenario & 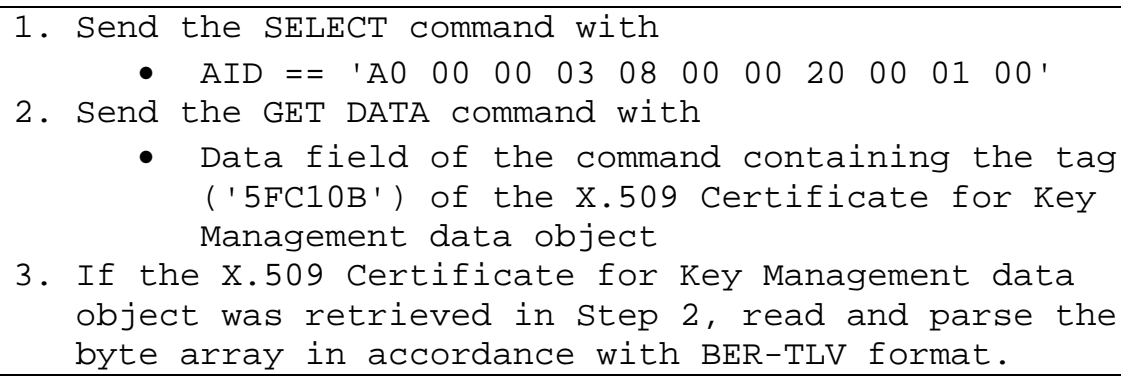 \\
\hline
\end{tabular}




\section{Expected Result(s)}

1. From Step 1, the command returns the application property template with the status word '90 00'.

2. From Step 2, the command returns one of the following:

- An X.509 Certificate for Key Management followed by status word '90 00';

- A zero-length data object followed by status word '90 00' which indicates that a container for the X.509 certificate exists, but it has not been personalized; or

- Status word '6A 82' (data object not found) - which indicates the container for the X.509 certificate does not exist

3. From Step 3, all mandatory tags for the X.509 Certificate for Key Management are present in the order indicated in Table 16 of [SP800-73], Part 1.

\subsubsection{Discovery Object}

\subsubsection{BER-TLV of Discovery Object and Presence of Security Object}

\begin{tabular}{|c|c|}
\hline Test Assertion & TA-09.01.04.01 \\
\hline Purpose & $\begin{array}{l}\text { Verifies that the Discovery Object (if present) conforms to the } \\
\text { Derived PIV data model requirements. }\end{array}$ \\
\hline DTR(s) & $\begin{array}{ll}\text { - } & \text { DTR-07.01.01.01 } \\
\text { - } & \text { DTR-07.01.05.01 } \\
\text { - } & \text { DTR-07.01.05.02 } \\
\text { - } & \text { DTR-07.01.08.02 }\end{array}$ \\
\hline Issuer Documentation & None. \\
\hline Precondition(s) & $\begin{array}{l}\text { - A token with the Derived PIV Application is inserted into an } \\
\text { appropriate token reader. } \\
\text { - Suitable drivers have been loaded between the test system and } \\
\text { an instance of the reader. }\end{array}$ \\
\hline Test Scenario & $\begin{array}{l}\text { 1. Send the SELECT token command with } \\
\bullet \text { AID == 'A } \odot \odot \odot \odot \odot 3 \odot 8 \odot \odot \odot \odot 2 \odot \odot \odot \odot 1 \odot \odot \text { ' } \\
\text { 2. Send the GET DATA command with } \\
\text { - Data field of the command containing the tag } \\
\text { ('7E') of the Discovery Object. } \\
\text { 3. If the Discovery Object was retrieved in Step 2, } \\
\text { read and parse the byte array in accordance with } \\
\text { BER-TLV format. } \\
\text { 4. If the Discovery object was retrieved in Step 2, } \\
\text { verify that the Security object is present within } \\
\text { the Derived PIV Application by sending a GET DATA } \\
\text { command to read the data object. }\end{array}$ \\
\hline Expected Result(s) & $\begin{array}{l}\text { 1. From Step 1, the command returns the application property } \\
\text { template with the status word ' } 9000 \text { '. } \\
\text { 2. From Step 2, the command returns one of the following: }\end{array}$ \\
\hline
\end{tabular}




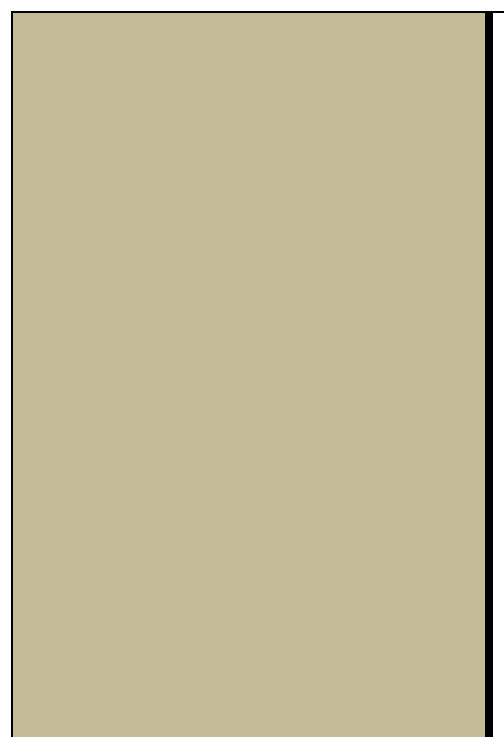

- A Discovery Object followed by status word '90 00';

- A zero-length data object followed by status word '90 00' which indicates that a container for the Discovery Object exists, but it has not been personalized; or

- Status word '6A 82' (data object not found) - which indicates the container for the Discovery Object does not exist)

3. From Step 3, all mandatory tags for the Discovery Object are present and are in the order indicated in Table 18 of [SP80073], Part 1. The first byte of the PIN Usage Policy is set to $0 \times 40$ and the second byte is set to $0 x 00$. In addition, the PIV Card Application AID in tag 0x4F is set to 'A0 0000030800 0020000100 '

4. From Step 4, the command returns status word '90 00' along with the Security Object.

\subsubsection{Key History Object}

\subsubsection{BER-TLV of Key History Object and Presence of Security Object}

\begin{tabular}{|c|c|}
\hline Test Assertion & TA-09.01.05.01 \\
\hline Purpose & $\begin{array}{l}\text { Verifies that the Key History Object (if present) conforms to the } \\
\text { Derived PIV data model requirements. }\end{array}$ \\
\hline DTR(s) & $\begin{array}{ll} & \text { DTR-07.01.01.01 } \\
- & \text { DTR-07.01.06.01 } \\
- & \text { DTR-07.01.08.02 } \\
\end{array}$ \\
\hline Issuer Documentation & None. \\
\hline Precondition(s) & $\begin{array}{l}\text { A token with the Derived PIV Application is inserted into an } \\
\text { appropriate token reader. } \\
\text { - Suitable drivers have been loaded between the test system and } \\
\text { an instance of the reader. }\end{array}$ \\
\hline Test Scenario & 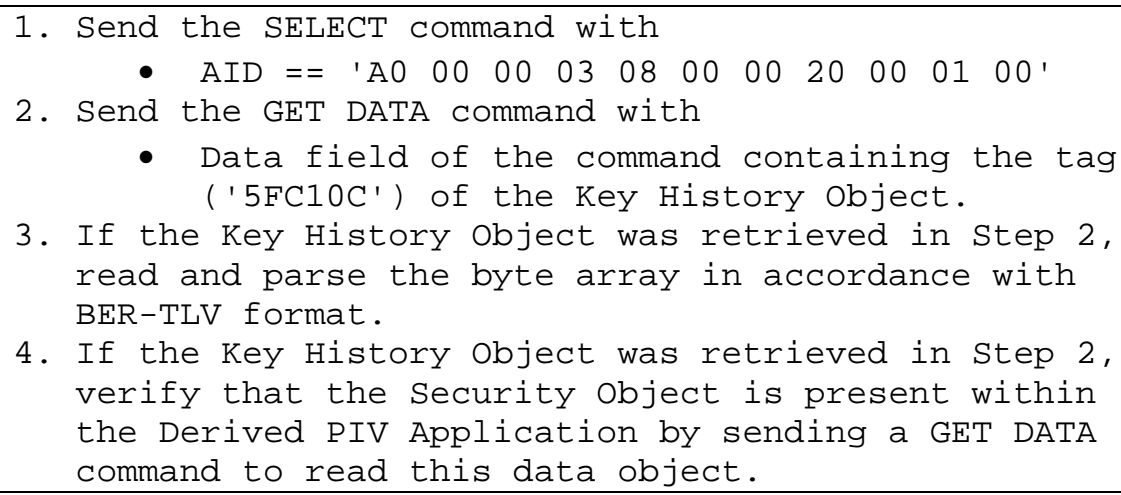 \\
\hline Expected Result(s) & $\begin{array}{l}\text { 1. From Step 1, the command returns the application property } \\
\text { template with the status word ' } 9000 \text { '. } \\
\text { 2. From Step 2, the command returns one of the following: }\end{array}$ \\
\hline
\end{tabular}




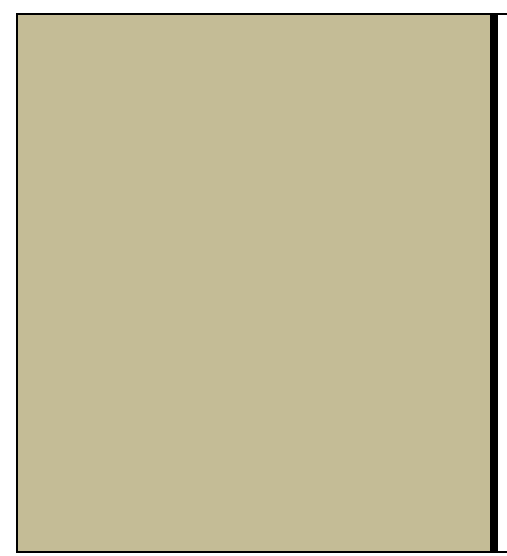

- A Key History Object followed by status word ' 90 00';

- A zero-length data object followed by status word '90 00' which indicates that a container for the Key History Object exists, but it has not been personalized; or

- Status word '6A 82' (data object not found) - which indicates the container for the Key History Object does not exist

3. From Step 3, all mandatory tags for the Key History Object are present in the order indicated in Table 19 of [SP800-73], Part 1.

4. From Step 4, the command returns status word ' 90 00' along with the Security Object.

\subsubsection{Retired X.509 Certificates for Key Management}

\subsubsection{BER-TLV of Retired X.509 Certificates for Key Management}

\begin{tabular}{|c|c|}
\hline Test Assertion & TA-09.01.06.01 \\
\hline Purpose & $\begin{array}{l}\text { Verifies that the Retired X.509 Certificates for Key Management } \\
\text { (if present) conform to the Derived PIV data model requirements. }\end{array}$ \\
\hline DTR(s) & $\begin{array}{l}-\quad \text { DTR-07.01.01.01 } \\
\text { - DTR-07.01.07.01 }\end{array}$ \\
\hline Issuer Documentation & None. \\
\hline Precondition(s) & $\begin{array}{l}\text { - A token with the Derived PIV Application is inserted into an } \\
\text { appropriate token reader. } \\
\text { - Suitable drivers have been loaded between the test system and } \\
\text { an instance of the reader. }\end{array}$ \\
\hline Test Scenario & 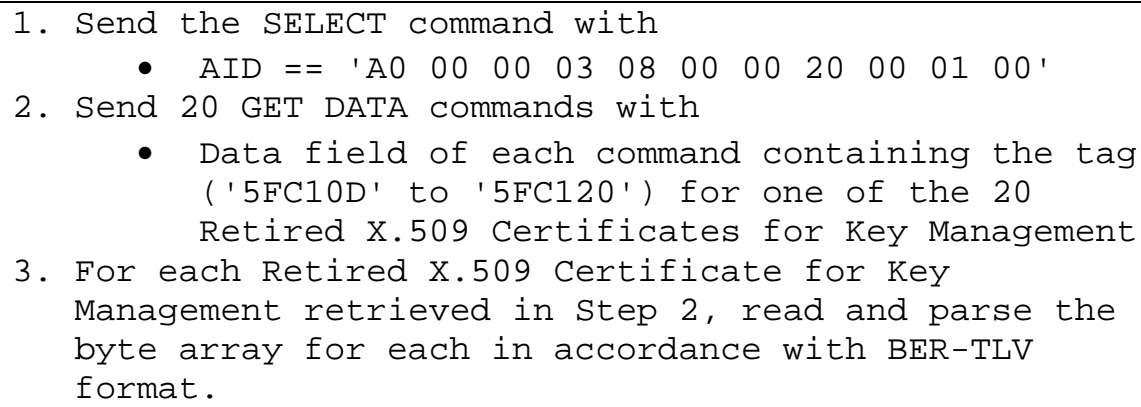 \\
\hline Expected Result(s) & $\begin{array}{l}\text { 1. From Step 1, the command returns the application property } \\
\text { template with the status word ' } 9000 \text { '. } \\
\text { 2. From Step 2, each command returns one of the following: } \\
\text { - A Retired X.509 Certificate for Key Management followed } \\
\text { by status word ' } 9000 \text { '; } \\
\text { - A zero-length data object followed by status word '90 00' - } \\
\text { which indicates that a container for the retired X.509 } \\
\text { certificate exists, but it has not been personalized; or } \\
\text { - Status word '6A 82' (data object not found) - which }\end{array}$ \\
\hline
\end{tabular}




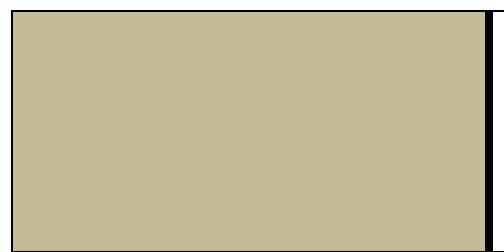

indicates the container for the retired X.509 certificate does not exist)

3. From Step 3, all mandatory tags in each of the available Retired X.509 Certificates for Key Management are present in the order indicated in Table 20 to Table 39 of [SP800-73], Part 1.

\subsubsection{Security Object}

\subsubsection{BER-TLV of Security Object and Presence of Unsigned Data Objects}

\begin{tabular}{|c|c|}
\hline Test Assertion & TA-09.01.07.01 \\
\hline Purpose & $\begin{array}{l}\text { Verifies that the Security Object conforms to the Derived PIV data } \\
\text { model requirements and unsigned data objects are included within } \\
\text { the Security Object on the Derived PIV Application. }\end{array}$ \\
\hline DTR(s) & $\begin{array}{ll} & \text { DTR-07.01.01.01 } \\
\text { - } & \text { DTR-07.01.08.01 } \\
\text { - } & \text { DTR-07.01.08.03 }\end{array}$ \\
\hline Issuer Documentation & None. \\
\hline Precondition(s) & $\begin{array}{l}\text { - A token with the Derived PIV Application is inserted into an } \\
\text { appropriate token reader. } \\
\text { - Suitable drivers have been loaded between the test system and } \\
\text { an instance of the reader. } \\
\text { - A Security Object is present within the Derived PIV } \\
\text { Application. }\end{array}$ \\
\hline Test Scenario & 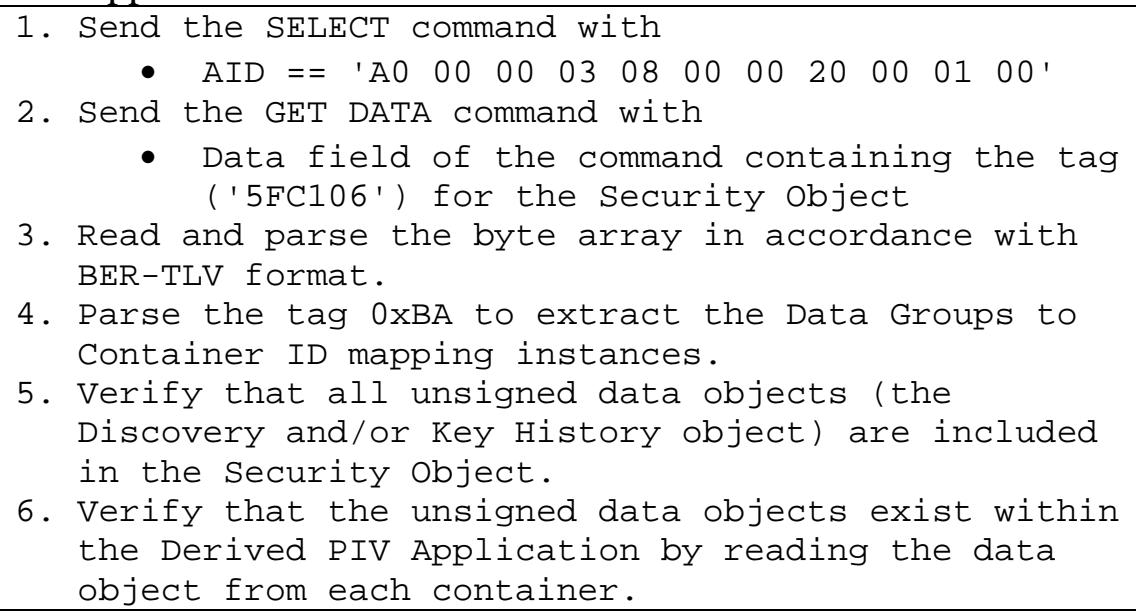 \\
\hline Expected Result(s) & $\begin{array}{l}\text { 1. From Step 1, the command returns the application property } \\
\text { template with the status word ' } 9000 \text { '. } \\
\text { 2. From Step 2, the command returns the Security Object } \\
\text { followed by status word ' } 9000 \text { '. } \\
\text { 3. From Step 3, all mandatory tags for the Security Object are } \\
\text { present in the order indicated in Table } 12 \text { of [SP800-73], Part } 1 .\end{array}$ \\
\hline
\end{tabular}


4. From Step 5, all unsigned data objects are included in the Security Object.

5. From Step 6, all data objects found in the mapping are actually present on the Derived PIV Application as evidenced by the GET DATA commands returning the data objects along with status word '90 00'.

\subsection{Signed Data Object Conformance}

\subsubsection{Security Object}

\subsubsection{Data Object Hash Integrity Check}

\begin{tabular}{|c|c|}
\hline Test Assertion & TA-09.02.01.01 \\
\hline Purpose & $\begin{array}{l}\text { Verifies the integrity of the hashes of the data objects referenced in } \\
\text { the Security Object (if present). }\end{array}$ \\
\hline DTR(s) & $\begin{array}{ll} & \text { DTR-07.02.01.01 } \\
\text { - } & \text { DTR-07.01.05.02 } \\
\text { - } & \text { DTR-07.01.08.02 } \\
\end{array}$ \\
\hline Issuer Documentation & None. \\
\hline Precondition(s) & $\begin{array}{l}\text { - A token with the Derived PIV Application is inserted into an } \\
\text { appropriate token reader. } \\
\text { - Suitable drivers have been loaded between the test system and } \\
\text { an instance of the reader. } \\
\text { - A Security Object is present within the Derived PIV } \\
\text { Application. }\end{array}$ \\
\hline Test Scenario & 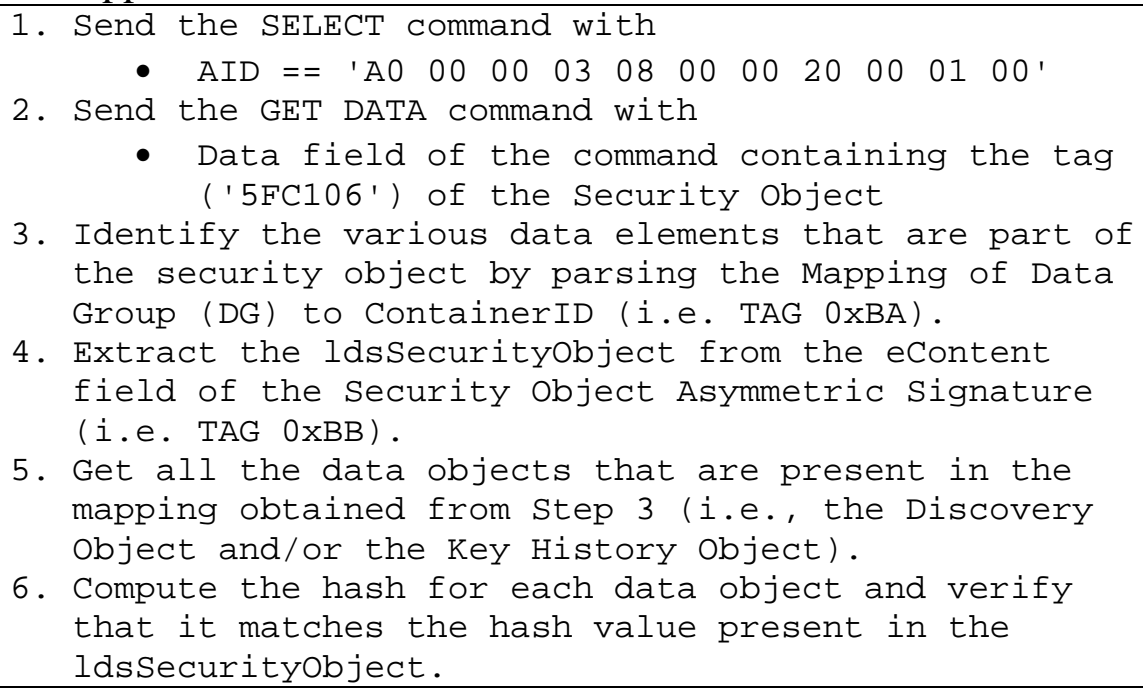 \\
\hline Expected Result(s) & $\begin{array}{l}\text { 1. From Step 1, the command returns the application property } \\
\text { template with the status word ' } 9000 \text { '. } \\
\text { 2. From Step 2, the command returns the Security Object and the } \\
\text { status word ' } 9000 \text { '. }\end{array}$ \\
\hline
\end{tabular}


3. From Step 5, the command returns the Discovery Object and/or Key History Object.

4. From Step 6, the actual hashes of the data objects extracted in Step 5 are identical to their corresponding hash values present in the Security Object.

\subsubsection{Presence of CMS SignedData}

\begin{tabular}{|c|c|}
\hline Test Assertion & TA-09.02.01.02 \\
\hline Purpose & $\begin{array}{l}\text { Verifies that the Security Object contains an asymmetric digital } \\
\text { signature, implemented as a SignedData type in accordance with } \\
\text { [RFC5652]. }\end{array}$ \\
\hline DTR(s) & $\begin{array}{ll}- & \text { DTR-07.02.01.02 } \\
\text { - } & \text { DTR-07.02.01.03 } \\
\end{array}$ \\
\hline Issuer Documentation & None. \\
\hline Precondition(s) & $\begin{array}{l}\text { - A token with the Derived PIV Application is inserted into an } \\
\text { appropriate token reader. } \\
\text { - Suitable drivers have been loaded between the test system and } \\
\text { an instance of the reader. } \\
\text { - A Security Object is present within the Derived PIV } \\
\text { Application. }\end{array}$ \\
\hline Test Scenario & 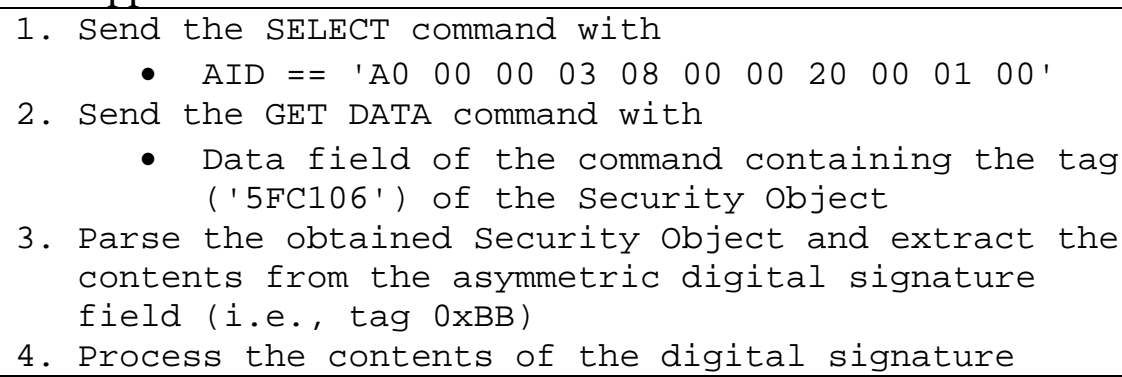 \\
\hline Expected Result(s) & $\begin{array}{l}\text { 1. From Step 1, the command returns the application property } \\
\text { template with the status word ' } 9000 \text { '. } \\
\text { 2. From Step 2, the command returns the requested data object } \\
\text { along with the status word ' } 9000 \text { '. } \\
\text { 3. From Step 4, the content of the digital signature is an object } \\
\text { that is a SignedData type which is in accordance with } \\
\text { [RFC5652]. }\end{array}$ \\
\hline
\end{tabular}

\subsubsection{SignedData Version}

\begin{tabular}{|l|l|}
\hline Test Assertion & TA-09.02.01.03 \\
\hline Purpose & Verifies that the version of the SignedData content type is v3. \\
\hline DTR(s) & $\bullet \quad$ DTR-07.02.01.04 \\
\hline Issuer Documentation & None. \\
\hline Precondition(s) & $\begin{array}{l}\text { A token with the Derived PIV Application is inserted into an } \\
\text { appropriate token reader. }\end{array}$ \\
\hline
\end{tabular}




\begin{tabular}{|c|c|}
\hline & $\begin{array}{l}\text { - Suitable drivers have been loaded between the test system and } \\
\text { an instance of the reader. } \\
\text { - A Security Object is present within the Derived PIV } \\
\text { Application. }\end{array}$ \\
\hline Test Scenario & 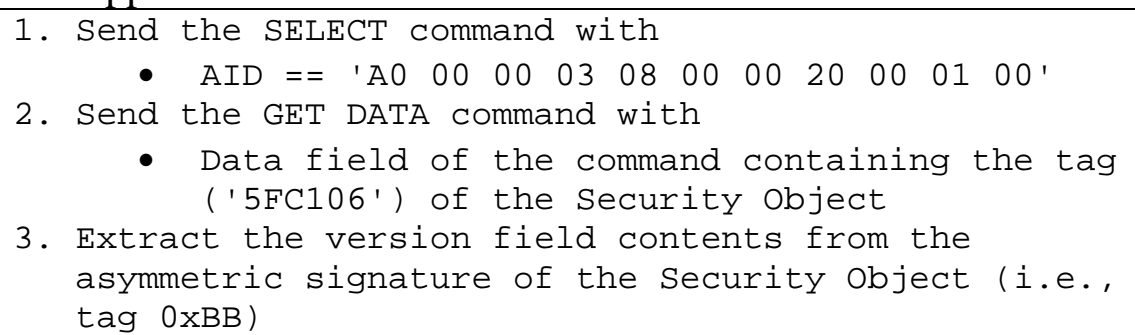 \\
\hline Expected Result(s) & $\begin{array}{l}\text { 1. From Step 1, the command returns the application property } \\
\text { template with the status word ' } 9000 \text { '. } \\
\text { 2. From Step 2, the command returns the requested data object } \\
\text { along with the status word ' } 9000 \text { '. } \\
\text { 3. From Step 3, the value of the version field of the SignedData is } \\
\text { v3. }\end{array}$ \\
\hline
\end{tabular}

\subsubsection{SignedData digestAlgorithms}

\begin{tabular}{|c|c|}
\hline Test Assertion & TA-09.02.01.04 \\
\hline Purpose & $\begin{array}{l}\text { Verifies that the digestAlgorithms field of the SignedData content } \\
\text { type is in accordance with Table 3-2 of [SP800-78]. }\end{array}$ \\
\hline DTR(s) & - $\quad$ DTR-07.02.01.05 \\
\hline Issuer Documentation & None. \\
\hline Precondition(s) & $\begin{array}{l}\text { - A token with the Derived PIV Application is inserted into an } \\
\text { appropriate token reader. } \\
\text { - Suitable drivers have been loaded between the test system and } \\
\text { an instance of the reader. } \\
\text { - A Security Object is present within the Derived PIV } \\
\text { Application. }\end{array}$ \\
\hline Test Scenario & 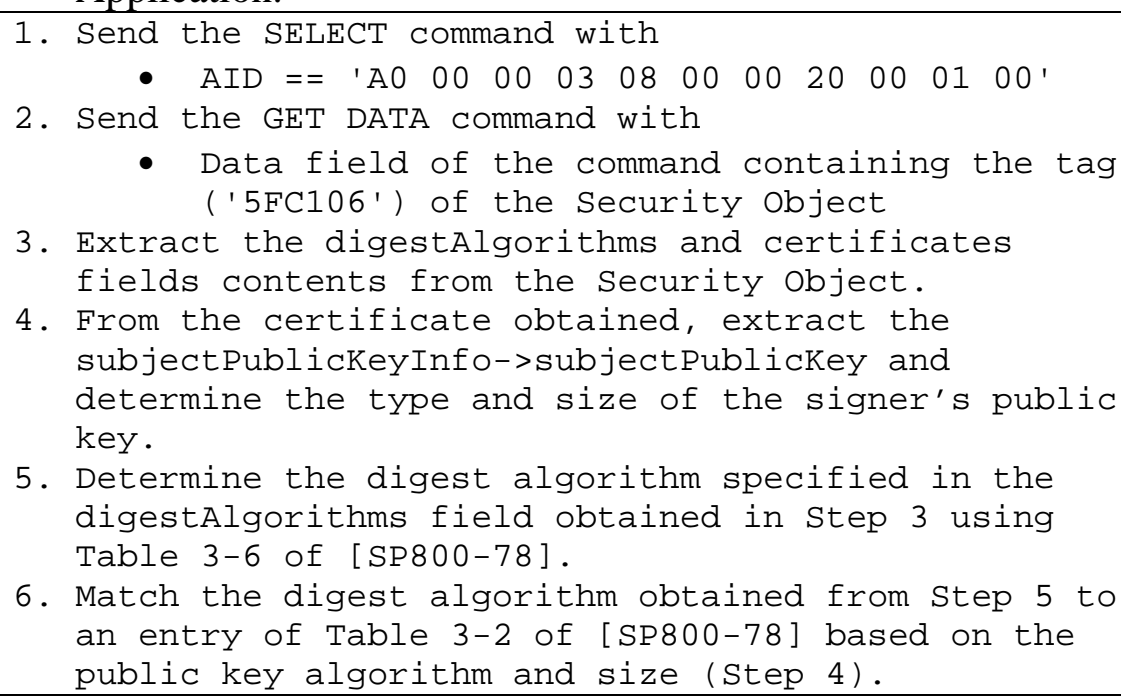 \\
\hline
\end{tabular}


Expected Result(s)

1. From Step 1, the command returns the application property template with the status word ' 90 00'.

2. From Step 2, the command returns the requested data object along with the status word '90 00'.

3. From Step 6, the digestAlgorithms field value of the SignedData is in accordance with Table 3-2 of [SP800-78].

\subsubsection{5 encapContentInfo Contents}

\begin{tabular}{|c|c|}
\hline Test Assertion & TA-09.02.01.05 \\
\hline Purpose & $\begin{array}{l}\text { Verifies that the eContentType of the encapContentInfo is id-icao- } \\
\text { ldsSecurityObject and the eContent field of the encapContentInfo } \\
\text { contains the contents of the ldsSecurity object. }\end{array}$ \\
\hline DTR(s) & $\begin{array}{ll} & \text { DTR-07.02.01.06 } \\
- & \text { DTR-07.02.01.07 } \\
\end{array}$ \\
\hline Issuer Documentation & None. \\
\hline Precondition(s) & $\begin{array}{l}\text { - A token with the Derived PIV Application is inserted into an } \\
\text { appropriate token reader. } \\
\text { - Suitable drivers have been loaded between the test system and } \\
\text { an instance of the reader. } \\
\text { - A Security Object is present within the Derived PIV } \\
\text { Application. }\end{array}$ \\
\hline Test Scenario & 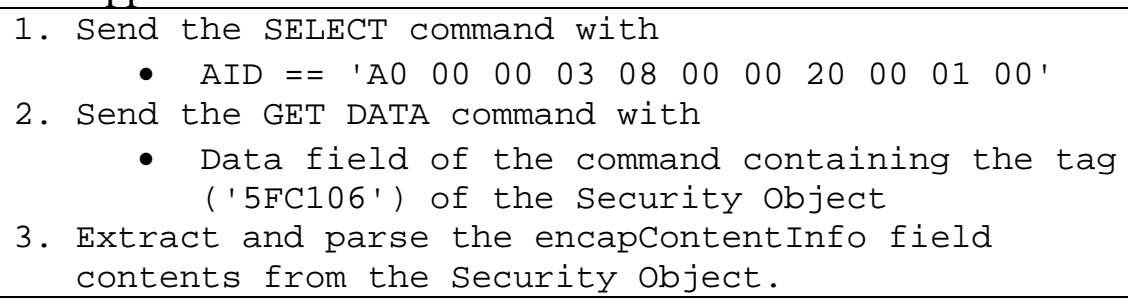 \\
\hline Expected Result(s) & $\begin{array}{l}\text { 1. From Step 1, the command returns the application property } \\
\text { template with the status word ' } 9000 \text { '. } \\
\text { 2. From Step 2, the command returns the requested data object } \\
\text { along with the status word ' } 9000 \text { '. } \\
\text { 3. From Step 3, the eContent field contains a correctly formatted } \\
\text { ldsSecurityobject and the eContentType asserts id-icao- } \\
\text { ldsSecurityObject in encapContentInfo. }\end{array}$ \\
\hline
\end{tabular}

\subsubsection{Derived PIV Credential Issuer's (Content Signing) Certificate Inclusion}

\begin{tabular}{|c|c|}
\hline Test Assertion & TA-09.02.01.06 \\
\hline Purpose & $\begin{array}{l}\text { Verifies that the Security Object includes the certificate of the } \\
\text { Derived PIV Credential Issuer (i.e., the issuer's content signing } \\
\text { certificate). }\end{array}$ \\
\hline DTR(s) & • $\quad$ DTR-07.02.01.08 \\
\hline Issuer Documentation & None. \\
\hline
\end{tabular}




\begin{tabular}{|l|l|}
\hline Precondition(s) & $\begin{array}{l}\text { A token with the Derived PIV Application is inserted into an } \\
\text { appropriate token reader. } \\
\text { - }\end{array}$ \\
\hline Suitable drivers have been loaded between the test system and \\
an instance of the reader. \\
A Aecurity Object is present within the Derived PIV \\
Application.
\end{tabular}

\subsubsection{SignerInfo digestAlgorithm}

\begin{tabular}{|l|l|}
\hline Test Assertion & TA-09.02.01.07 \\
\hline Purpose & $\begin{array}{l}\text { Verifies that the digestAlgorithm field of the SignerInfo field is in } \\
\text { accordance with Table 3-2 of [SP800-78]. }\end{array}$ \\
\hline DTR(s) & - DTR-07.02.01.09 \\
\hline Issuer Documentation & None. \\
\hline Precondition(s) & $\begin{array}{l}\text { A token with the Derived PIV Application is inserted into an } \\
\text { appropriate token reader. }\end{array}$ \\
- Suitable drivers have been loaded between the test system and \\
an instance of the reader. \\
- A Security Object is present within the Derived PIV \\
Application.
\end{tabular}




\begin{tabular}{|l|l|}
\hline & $\begin{array}{l}\text { 7. Determine the digest algorithm specified in the } \\
\text { digestAlgorithm field obtained in Step } 3 \text { using } \\
\text { Table } 3-6 \text { of [SP800-78] } \\
\text { 8. Match the digest algorithm obtained from Step } 7 \text { to } \\
\text { an entry of Table } 3-2 \text { of [SP800-78] based on the } \\
\text { public key algorithm and size (Step 6). }\end{array}$ \\
\hline Expected Result(s) & $\begin{array}{l}\text { From Step 1, the command returns the application property } \\
\text { template with the status word '90 00'. }\end{array}$ \\
2. From Step 2, the command the requested data object along with \\
the returns status word '90 00'. \\
3. From Step 8, the digestAlgorithm field value of the SignerInfo \\
is in accordance with Tables 3-6 and 3-2 of [SP800-78] and it \\
matches the value present in the digestAlgorithms field of the \\
SignedData.
\end{tabular}

\subsubsection{SignerInfo signatureAlgorithm}

\begin{tabular}{|c|c|}
\hline Test Assertion & TA-09.02.01.08 \\
\hline Purpose & $\begin{array}{l}\text { Verifies that for RSA with PKCS \#1 v1.5 padding, the } \\
\text { signatureAlgorithm field specifies the rsaEncryption OID (as per } \\
\text { Section } 3.2 \text { of [RFC3370]) and for ECDSA and RSA with PSS } \\
\text { padding, the signatureAlgorithm is in accordance with Table 3-3 of } \\
\text { [SP800-78]. }\end{array}$ \\
\hline DTR(s) & - $\quad$ DTR-07.02.01.10 \\
\hline Issuer Documentation & None. \\
\hline Precondition(s) & $\begin{array}{l}\text { - A token with the Derived PIV Application is inserted into an } \\
\text { appropriate token reader. } \\
\text { - Suitable drivers have been loaded between the test system and } \\
\text { an instance of the reader. } \\
\text { - A Security Object is present within the Derived PIV } \\
\text { Application. }\end{array}$ \\
\hline Test Scenario & 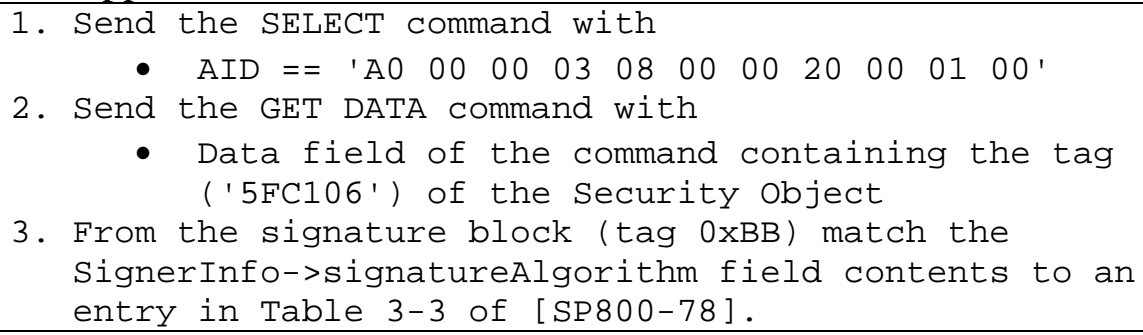 \\
\hline Expected Result(s) & $\begin{array}{l}\text { 1. From Step 1, the command returns the application property } \\
\text { template with the status word ' } 9000 \text { '. } \\
\text { 2. From Step 2, the command returns the requested data object } \\
\text { along with the status word ' } 9000 \text { '. } \\
\text { 3. From Step 3, the signatureAlgorithm field specified in the } \\
\text { SignerInfo field for RSA with PKCS \#1 v1.5 padding specifies } \\
\text { the rsaEncryption OID (as per Section } 3.2 \text { of [RFC3370]) and } \\
\text { for ECDSA and RSA with PSS padding, the }\end{array}$ \\
\hline
\end{tabular}


signatureAlgorithm is in accordance with Table 3-3 of [SP80078].

\subsubsection{Digital Signature}

\begin{tabular}{|c|c|}
\hline Test Assertion & TA-09.02.01.09 \\
\hline Purpose & $\begin{array}{l}\text { Verifies that the signature in the SignerInfo corresponds to the } \\
\text { Security Object and that it is signed with the Derived PIV } \\
\text { Credential Issuer's (content signing) certificate. }\end{array}$ \\
\hline DTR(s) & - DTR-07.02.01.11 \\
\hline Issuer Documentation & None. \\
\hline Precondition(s) & $\begin{array}{l}\text { - A token with the Derived PIV Application is inserted into an } \\
\text { appropriate token reader. } \\
\text { - Suitable drivers have been loaded between the test system and } \\
\text { an instance of the reader. } \\
\text { - A Security Object is present within the Derived PIV } \\
\text { Application. }\end{array}$ \\
\hline Test Scenario & 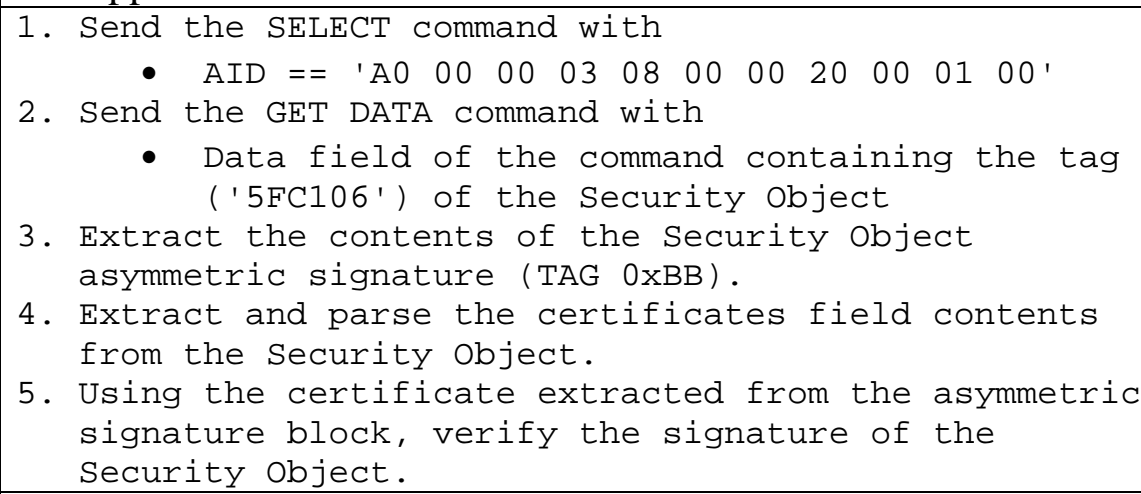 \\
\hline Expected Result(s) & $\begin{array}{l}\text { 1. From Step 1, the command returns the application property } \\
\text { template with the status word ' } 9000 \text { '. } \\
\text { 2. From Step 2, the command returns the requested data object } \\
\text { along with the status word ' } 9000 \text { '. } \\
\text { 3. From Step 5, the certificates field of the SignedData contains } \\
\text { the Derived PIV Credential Issuer's (content signing) } \\
\text { certificate, which is used to verify the digital signature on the } \\
\text { Security Object. }\end{array}$ \\
\hline
\end{tabular}




\subsection{PKI Conformance}

\subsubsection{X.509 Certificate for Derived PIV Authentication ${ }^{18}$}

\subsubsection{Signature Algorithm}

\begin{tabular}{|c|c|}
\hline Test Assertion & TA-09.03.01.01 \\
\hline Purpose & $\begin{array}{l}\text { Verifies that the proper signature algorithm has been used to sign } \\
\text { the Derived PIV Authentication certificate as specified in Table 3-3 } \\
\text { of [SP800-78]. }\end{array}$ \\
\hline DTR(s) & $\begin{array}{ll}- & \text { DTR-07.03.01.01 } \\
\text { - } & \text { DTR-07.03.01.02 }\end{array}$ \\
\hline Issuer Documentation & None. \\
\hline Precondition(s) & $\begin{array}{l}\text { - A token with the Derived PIV Application is inserted into an } \\
\text { appropriate token reader. } \\
\text { - Suitable drivers have been loaded between the test system and } \\
\text { an instance of the reader. } \\
\text { - A Derived PIV Authentication certificate is present within the } \\
\text { Derived PIV Application. }\end{array}$ \\
\hline Test Scenario & 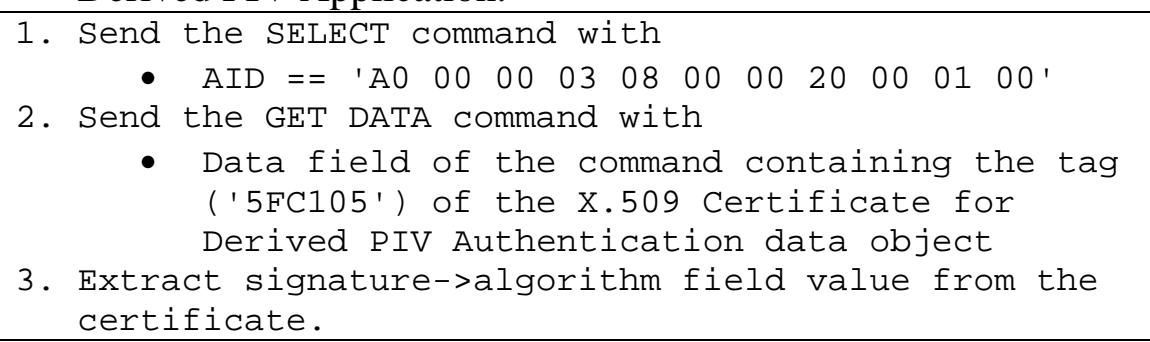 \\
\hline Expected Result(s) & $\begin{array}{l}\text { 1. From Step 1, the command returns the application property } \\
\text { template with the status word ' } 9000 \text { '. } \\
\text { 2. From Step 2, the command returns the requested data object } \\
\text { along with the status word ' } 9000 \text { ' } \\
\text { 3. From Step 3, the algorithm value is in accordance with Table 3- } \\
3 \text { of [SP800-78]. If the algorithm value is id-RSASSA-PSS, } \\
\text { then the hashAlgorithm field in signature->parameters is } \\
\text { populated with SHA-256 (OID = 2.16.840.1.101.3.4.2.1). For } \\
\text { RSA with PKCS \#1 v1.5 padding, the parameters field is } \\
\text { populated with NULL. For ECDSA, the parameters field is } \\
\text { absent. }\end{array}$ \\
\hline
\end{tabular}

\footnotetext{
${ }^{18}$ The Derived PIV Authentication key and certificate may be tested outside of the Derived PIV Application. Specific test assertions can be developed by test entities to test this key and certificate based on the environment (e.g., web browser) in which the key pair is being used. See Appendix A for examples of testing approaches.
} 


\subsubsection{Subject Public Key Algorithm}

\begin{tabular}{|c|c|}
\hline Test Assertion & TA-09.03.01.02 \\
\hline Purpose & $\begin{array}{l}\text { Verifies that the public key algorithm used for generating the keys } \\
\text { is as specified in Table 3-4 of [SP800-78]. }\end{array}$ \\
\hline DTR(s) & $\begin{array}{ll}- & \text { DTR-07.03.01.03 } \\
\text { - } & \text { DTR-07.03.01.04 }\end{array}$ \\
\hline Issuer Documentation & None. \\
\hline Precondition(s) & $\begin{array}{l}\text { - A token with the Derived PIV Application is inserted into an } \\
\text { appropriate token reader. } \\
\text { - Suitable drivers have been loaded between the test system and } \\
\text { an instance of the reader. } \\
\text { - A Derived PIV Authentication certificate is present within the } \\
\text { Derived PIV Application. }\end{array}$ \\
\hline Test Scenario & 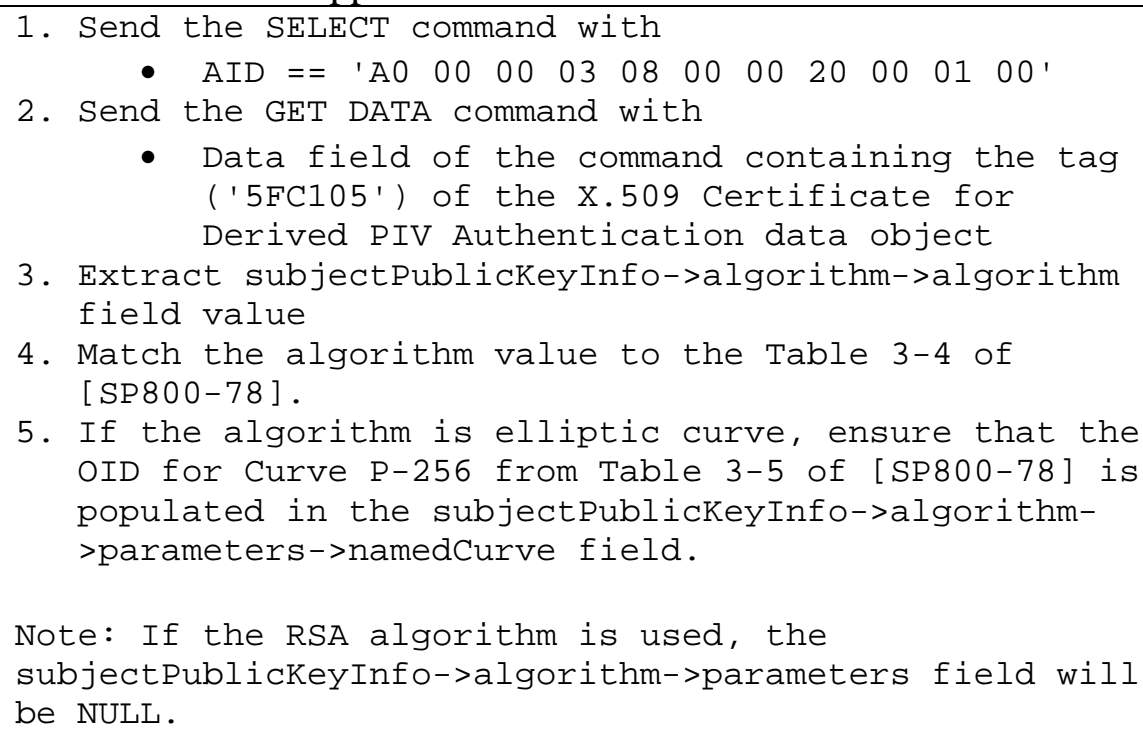 \\
\hline Expected Result(s) & $\begin{array}{l}\text { 1. From Step 1, the command returns the application property } \\
\text { template with the status word ' } 9000 \text { '. } \\
\text { 2. From Step 2, the command returns the requested data object } \\
\text { along with the status word ' } 9000 \text { '. } \\
\text { 3. From Steps } 4 \text { and 5, it is determined that the Derived PIV } \\
\text { Authentication key is generated using an allowed asymmetric } \\
\text { key algorithm. }\end{array}$ \\
\hline
\end{tabular}

\subsubsection{Public Key Size}

\begin{tabular}{|l|l|}
\hline Test Assertion & TA-09.03.01.03 \\
\hline Purpose & $\begin{array}{l}\text { Verifies that the key size requirements are in accordance with } \\
\text { Table 3-1 of [SP800-78]. }\end{array}$ \\
\hline DTR(s) & $\bullet \quad$ DTR-07.03.01.12 \\
\hline Issuer Documentation & None. \\
\hline
\end{tabular}




\begin{tabular}{|l|l|}
\hline Precondition(s) & $\begin{array}{l}\text { A token with the Derived PIV Application is inserted into an } \\
\text { appropriate token reader. } \\
\text { - }\end{array}$ \\
Suitable drivers have been loaded between the test system and \\
an instance of the reader. \\
A Derived PIV Authentication certificate is present within the \\
Derived PIV Application.
\end{tabular}

\subsubsection{Key Usage Extension}

\begin{tabular}{|c|c|}
\hline Test Assertion & TA-09.03.01.04 \\
\hline Purpose & $\begin{array}{l}\text { Verifies that the Derived PIV Authentication certificate asserts the } \\
\text { appropriate purpose for the key. }\end{array}$ \\
\hline $\operatorname{DTR}(\mathrm{s})$ & - DTR-07.03.01.05 \\
\hline Issuer Documentation & None. \\
\hline Precondition(s) & $\begin{array}{l}\text { - A token with the Derived PIV Application is inserted into an } \\
\text { appropriate token reader. } \\
\text { - Suitable drivers have been loaded between the test system and } \\
\text { an instance of the reader. } \\
\text { - A Derived PIV Authentication certificate is present within the } \\
\text { Derived PIV Application. }\end{array}$ \\
\hline Test Scenario & 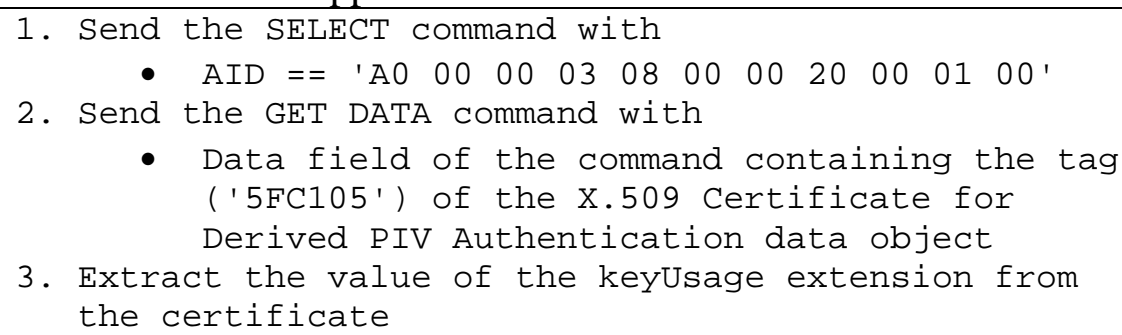 \\
\hline Expected Result(s) & $\begin{array}{l}\text { 1. From Step 1, the command returns the application property } \\
\text { template with the status word ' } 9000 \text { '. }\end{array}$ \\
\hline
\end{tabular}


2. From Step 2, the command returns the requested data object along with the status word '90 00'.

3. From Step 3, the digitalSignature bit has been set. No other bits have been set.

\subsubsection{Certificate Policy}

\begin{tabular}{|c|c|}
\hline Test Assertion & TA-09.03.01.05 \\
\hline Purpose & $\begin{array}{l}\text { Verifies that the Derived PIV Authentication certificate asserts the } \\
\text { appropriate certificate policy OID. }\end{array}$ \\
\hline DTR(s) & - $\quad$ DTR-07.03.01.06 \\
\hline Issuer Documentation & None. \\
\hline Precondition(s) & $\begin{array}{l}\text { - A token with the Derived PIV Application token is inserted } \\
\text { into an appropriate token reader. } \\
\text { - Suitable drivers have been loaded between the test system and } \\
\text { an instance of the reader. } \\
\text { - A Derived PIV Authentication certificate is present within the } \\
\text { Derived PIV Application. }\end{array}$ \\
\hline Test Scenario & 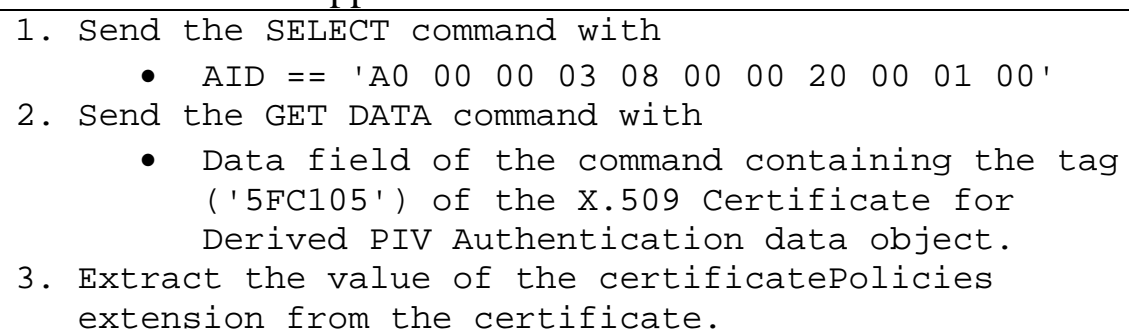 \\
\hline Expected Result(s) & $\begin{array}{l}\text { 1. From Step 1, the command returns the application property } \\
\text { template with the status word ' } 9000 \text { '. } \\
\text { 2. From Step 2, the command returns the requested data object } \\
\text { along with the status word ' } 9000 \text { '. } \\
\text { 3. From Step 3, the certificatePolicies extension asserts either the } \\
\text { id-fpki-common-derived-pivAuth or id-fpki-common-derived- } \\
\text { pivAuth-hardware OID. }\end{array}$ \\
\hline
\end{tabular}

\subsubsection{Authority Information Access Extension}

\begin{tabular}{|l|l|}
\hline Test Assertion & TA-09.03.01.06 \\
\hline Purpose & $\begin{array}{l}\text { Verifies that the authority information access extension in the } \\
\text { Derived PIV Authentication certificate is populated with: (i) the } \\
\text { location to the OCSP server that provides status information for } \\
\text { this certificate and (ii) the location to an HTTP accessible Web } \\
\text { server where certificates issued to the issuer of this certificate may } \\
\text { be found. }\end{array}$ \\
\hline DTR(s) & $\begin{array}{l}\text { - DTR-07.03.01.09 } \\
\text { - DTR-07.03.01.11 }\end{array}$ \\
\hline
\end{tabular}




\begin{tabular}{|c|c|}
\hline Issuer Documentation & None. \\
\hline Precondition(s) & $\begin{array}{l}\text { - A token with the Derived PIV Application is inserted into an } \\
\text { appropriate token reader. } \\
\text { - Suitable drivers have been loaded between the test system and } \\
\text { an instance of the reader. } \\
\text { - A Derived PIV Authentication certificate is present within the } \\
\text { Derived PIV Application. }\end{array}$ \\
\hline Test Scenario & 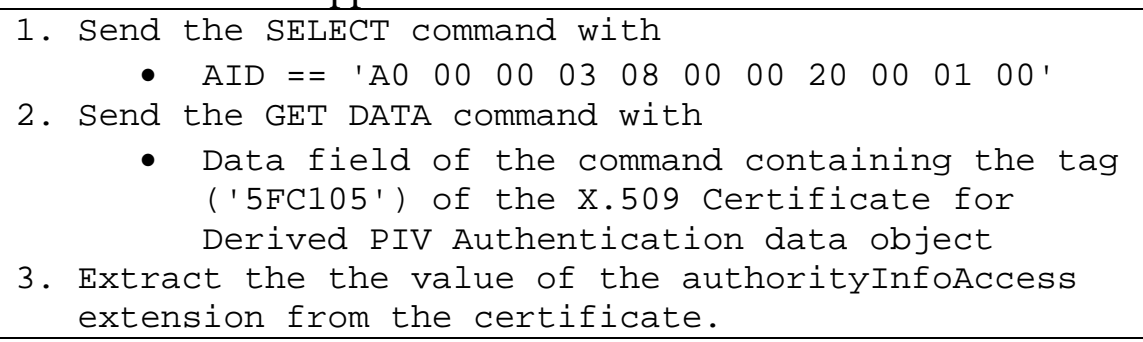 \\
\hline Expected Result(s) & $\begin{array}{l}\text { 1. From Step 1, the command returns the application property } \\
\text { template with the status word ' } 9000 \text { '. } \\
\text { 2. From Step 2, the command the requested data object along with } \\
\text { the returns status word '90 00'. } \\
\text { 3. From Step 3, an accessMethod containing id-ad-ocsp } \\
\text { (1.3.6.1.5.5.7.48.1) is present that contains an accessLocation } \\
\text { of type uniformResourceIdentifier where the scheme is "http" } \\
\text { (not "https"). An id-ad-caIssuers (1.3.6.1.5.5.7.48.2) } \\
\text { accessMethod is also present where the accessLocation is of } \\
\text { type uniformResourceIdentifier and the scheme is "http." }\end{array}$ \\
\hline
\end{tabular}

\subsubsection{Asymmetric Key Pair}

\begin{tabular}{|c|c|}
\hline Test Assertion & TA-09.03.01.07 \\
\hline Purpose & $\begin{array}{l}\text { Verifies that the public key that exists in the Derived PIV } \\
\text { Authentication certificate corresponds to the private key located in } \\
\text { the Derived PIV Application. }\end{array}$ \\
\hline DTR(s) & - $\quad$ DTR-07.03.01.13 \\
\hline Issuer Documentation & None. \\
\hline Precondition(s) & $\begin{array}{l}\text { - A token with the Derived PIV Application is inserted into an } \\
\text { appropriate token reader. } \\
\text { - Suitable drivers have been loaded between the test system and } \\
\text { an instance of the reader. } \\
\text { - A Derived PIV Authentication certificate is present within the } \\
\text { Derived PIV Application. } \\
\text { - The Derived PIV Application Password is recorded. } \\
\text { - The Derived PIV Application Password's retry counter is not } 0 .\end{array}$ \\
\hline Test Scenario & 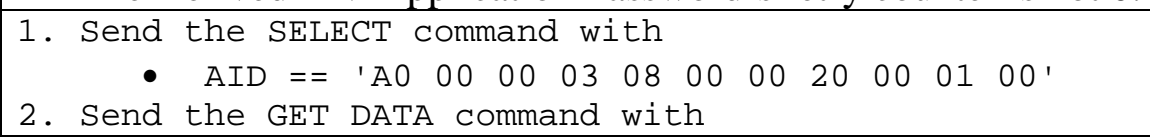 \\
\hline
\end{tabular}




\begin{tabular}{|c|c|}
\hline & 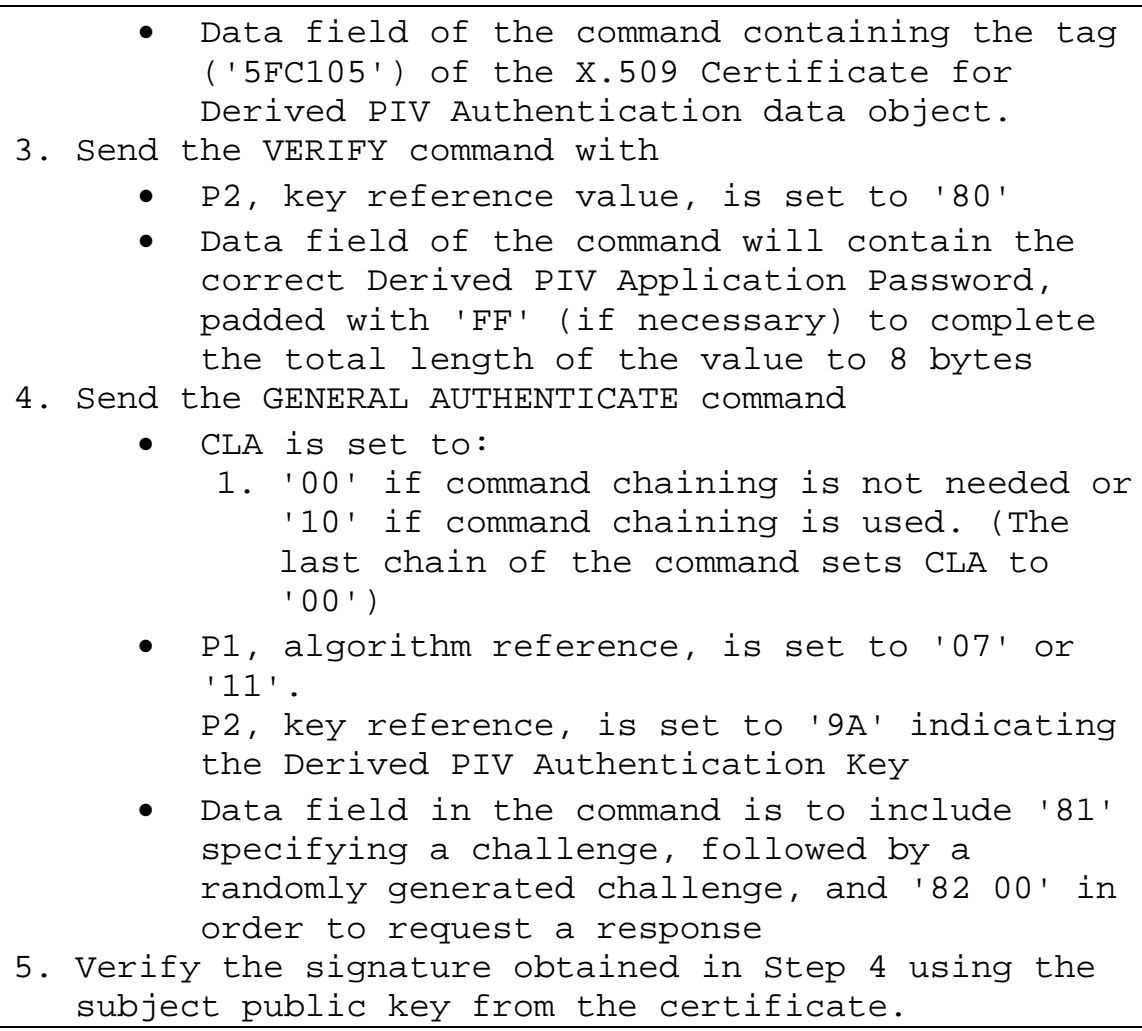 \\
\hline Expected Result(s) & $\begin{array}{l}\text { 1. From Step 1, the command returns the application property } \\
\text { template with the status word ' } 9000 \text { '. } \\
\text { 2. From Step 2, the command returns the requested data object } \\
\text { along with status word ' } 9000 \text { '. } \\
\text { 3. From Step 3, the command returns the status word ' } 9000 \text { '. } \\
\text { 4. From Step 4, the command returns the signed challenge with } \\
\text { the status word ' } 9000 \text { '. } \\
\text { 5. From Step 5, the private key corresponds to the public key } \\
\text { contained in the certificate as the signature verification } \\
\text { succeeds. }\end{array}$ \\
\hline
\end{tabular}

\subsubsection{UUID in the subjectAltName}

\begin{tabular}{|c|c|}
\hline Test Assertion & TA-09.03.01.08 \\
\hline Purpose & $\begin{array}{l}\text { Verifies that a UUID is populated in the subjectAltName field of } \\
\text { the Derived PIV Authentication certificate. }\end{array}$ \\
\hline DTR(s) & - $\quad$ DTR-07.03.01.07 \\
\hline Issuer Documentation & None. \\
\hline Precondition(s) & $\begin{array}{l}\text { - A token with the Derived PIV Application is inserted into an } \\
\text { appropriate token reader. } \\
\text { - Suitable drivers have been loaded between the test system and } \\
\text { an instance of the reader. } \\
\text { - A Derived PIV Authentication certificate is present within the } \\
\text { Derived PIV Application. }\end{array}$ \\
\hline
\end{tabular}




\begin{tabular}{|c|c|}
\hline Test Scenario & 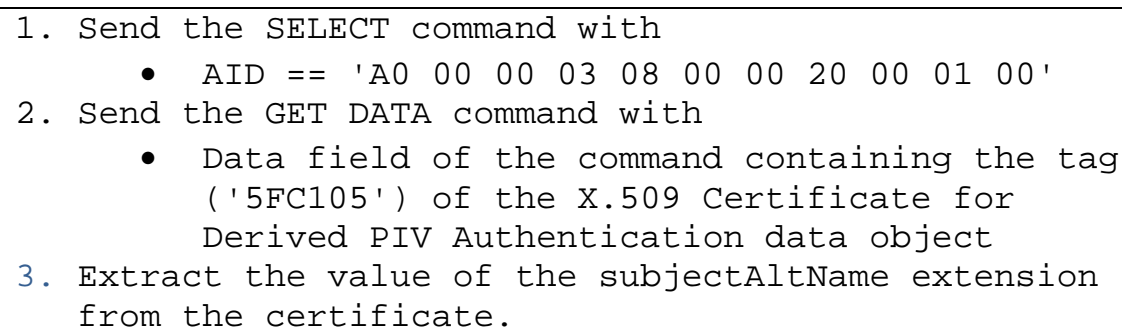 \\
\hline Expected Result(s) & $\begin{array}{l}\text { 1. From Step 1, the command returns the application property } \\
\text { template with the status word ' } 9000 \text { '. } \\
\text { 2. From Step 2, the command returns the requested data object } \\
\text { along with the status word ' } 9000 \text { '. } \\
\text { 3. From Step 3, a name of type uniformResourceIdentifier } \\
\text { containing a UUID is present. }\end{array}$ \\
\hline
\end{tabular}

\subsubsection{9 piv-interim Extension}

\begin{tabular}{|c|c|}
\hline Test Assertion & TA-09.03.01.09 \\
\hline Purpose & $\begin{array}{l}\text { Verifies that the piv-interim extension is present in the Derived } \\
\text { PIV Authentication certificate. }\end{array}$ \\
\hline DTR(s) & - $\quad$ DTR-07.03.01.08 \\
\hline Issuer Documentation & None. \\
\hline Precondition(s) & $\begin{array}{l}\text { - A token with the Derived PIV Application is inserted into an } \\
\text { appropriate token reader. } \\
\text { - Suitable drivers have been loaded between the test system and } \\
\text { an instance of the reader. } \\
\text { - A Derived PIV Authentication certificate is present within the } \\
\text { Derived PIV Application. }\end{array}$ \\
\hline Test Scenario & $\begin{array}{l}\text { 1. Send the SELECT command with } \\
\qquad \text { AID == 'A } \odot \odot \odot \odot \odot 3 \odot 8 \odot \odot \odot \odot 2 \odot \odot \odot \odot 1 \odot \odot \text { ' } \\
\text { 2. Send the GET DATA command with } \\
\text { - Data field of the command containing the tag } \\
\text { ('5FC105') of the X.509 Certificate for } \\
\text { Derived PIV Authentication data object } \\
\text { 3. Extract the piv-interim extension from the } \\
\text { certificate. }\end{array}$ \\
\hline Expected Result(s) & $\begin{array}{l}\text { 1. From Step 1, the command returns the application property } \\
\text { template with the status word ' } 9000 \text { '. } \\
\text { 2. From Step 2, the command returns the requested data object } \\
\text { along with the status word ' } 9000 \text { '. } \\
\text { 3. From Step 3, the non-critical piv-interim extension is present } \\
\text { and contains the interim_indicator field, which is of type } \\
\text { BOOLEAN. }\end{array}$ \\
\hline
\end{tabular}




\subsubsection{0 cRLDistributionPoints Extension}

\begin{tabular}{|c|c|}
\hline Test Assertion & TA-09.03.01.10 \\
\hline Purpose & $\begin{array}{l}\text { Verifies that the cRLDistributionPoints extension in the Derived } \\
\text { PIV Authentication certificate contains an HTTP URI. }\end{array}$ \\
\hline DTR(s) & - DTR-07.03.01.10 \\
\hline Issuer Documentation & None. \\
\hline Precondition(s) & $\begin{array}{l}\text { - A token with the Derived PIV Application is inserted into an } \\
\text { appropriate token reader. } \\
\text { - Suitable drivers have been loaded between the test system and } \\
\text { an instance of the reader. } \\
\text { - A Derived PIV Authentication certificate is present within the } \\
\text { Derived PIV Application. }\end{array}$ \\
\hline Test Scenario & 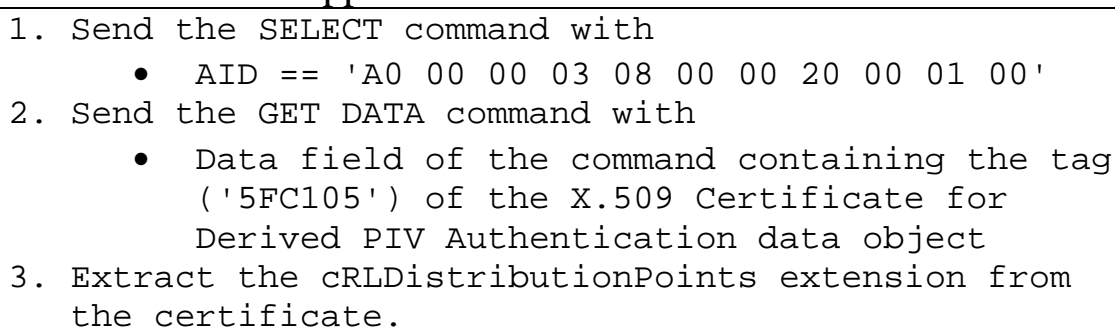 \\
\hline Expected Result(s) & $\begin{array}{l}\text { 1. From Step 1, the command returns the application property } \\
\text { template with the status word ' } 9000 \text { '. } \\
\text { 2. From Step 2, the command returns the requested data object } \\
\text { along with the status word ' } 9000 \text { '. } \\
\text { 3. From Step 3, a URI with the "HTTP" scheme that can be used } \\
\text { to access CRL information is present. }\end{array}$ \\
\hline
\end{tabular}

\subsubsection{RSA Exponent}

\begin{tabular}{|c|c|}
\hline Test Assertion & TA-09.03.01.11 \\
\hline Purpose & $\begin{array}{l}\text { Verifies that for RSA keys, the exponent of the asymmetric key for } \\
\text { Derived PIV Authentication is equal to } 65537 \text {. }\end{array}$ \\
\hline DTR(s) & - $\quad$ DTR-07.05.01.14 \\
\hline Issuer Documentation & None. \\
\hline Precondition(s) & $\begin{array}{l}\text { - A token with the Derived PIV Application is inserted into an } \\
\text { appropriate token reader. } \\
\text { - Suitable drivers have been loaded between the test system and } \\
\text { an instance of the reader. } \\
\text { - A Derived PIV Authentication certificate is present within the } \\
\text { Derived PIV Application. }\end{array}$ \\
\hline Test Scenario & 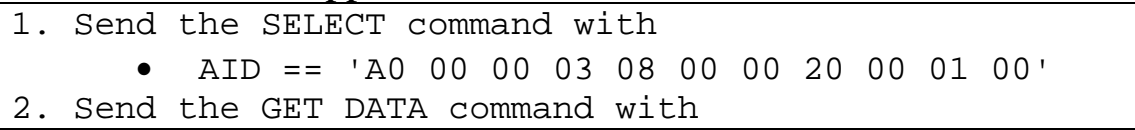 \\
\hline
\end{tabular}




\begin{tabular}{|l|l|}
\hline $\begin{array}{r}\text { Data field of the command containing the tag } \\
\text { ('5FC105') of the X.509 Certificate for } \\
\text { Derived PIV Authentication data object }\end{array}$ \\
$\begin{array}{l}\text { 3. Extract the subjectPublickeyInfo->subjectPublickey } \\
\text { from the certificate. } \\
\text { 4. Parse the exponent from the extracted public key. }\end{array}$ \\
$\begin{array}{l}\text { 1. Fxpected Result(s) } \\
\text { From Step 1, the command returns the application property } \\
\text { template with the status word '90 00'. }\end{array}$ \\
2. From Step 2, the command returns the requested data object \\
along with the status word '90 00'. \\
From Step 4, the exponent of the RSA asymmetric key for PIV \\
Authentication is equal to 65537.
\end{tabular}

\subsubsection{X.509 Certificate for Digital Signature ${ }^{19}$}

\subsubsection{Signature Algorithm}

\begin{tabular}{|c|c|}
\hline Test Assertion & TA-09.03.02.01 \\
\hline Purpose & $\begin{array}{l}\text { Verifies that the proper signature algorithm has been used to sign } \\
\text { the digital signature certificate as specified in Table 3-3 of [SP800- } \\
\text { 78]. }\end{array}$ \\
\hline DTR(s) & $\begin{array}{ll} & \text { DTR-07.03.02.01 } \\
\text { - } & \text { DTR-07.03.02.02 }\end{array}$ \\
\hline Issuer Documentation & None. \\
\hline Precondition(s) & $\begin{array}{l}\text { - A token with the Derived PIV Application is inserted into an } \\
\text { appropriate token reader. } \\
\text { - Suitable drivers have been loaded between the test system and } \\
\text { an instance of the reader. } \\
\text { - A digital signature certificate is present within the Derived PIV } \\
\text { Application. }\end{array}$ \\
\hline Test Scenario & 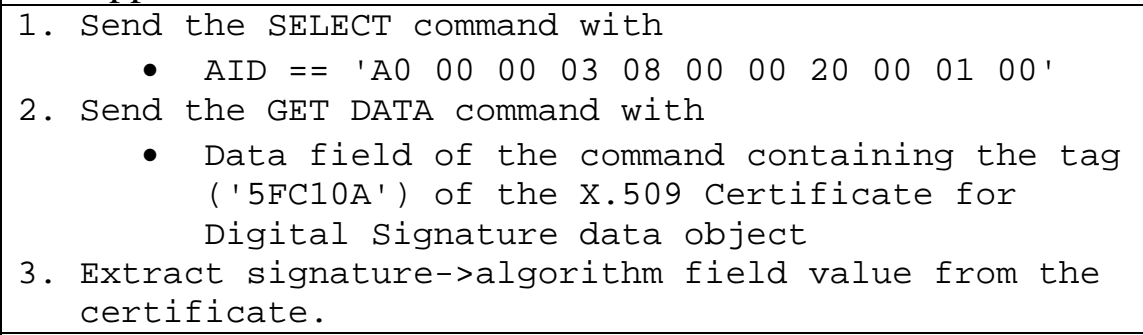 \\
\hline Expected Result(s) & $\begin{array}{l}\text { 1. From Step 1, the command returns the application property } \\
\text { template with the status word ' } 9000 \text { '. } \\
\text { 2. From Step 2, the command returns the requested data object } \\
\text { along with the status word ' } 9000 \text { '. }\end{array}$ \\
\hline
\end{tabular}

\footnotetext{
${ }^{19}$ The digital signature key and certificate may be tested outside of the Derived PIV Application. Specific test assertions can be developed by test entities to test this key and certificate based on the environment (e.g., email application) in which the key pair is being used. See Appendix A for example testing approaches.
} 
3. From Step 3, the algorithm value is in accordance with Table 33 of [SP800-78]. If the algorithm value is id-RSASSA-PSS, then the hashAlgorithm field in signature->parameters is populated with SHA-256 (OID = 2.16.840.1.101.3.4.2.1). For RSA with PKCS \#1 v1.5 padding, the parameters field is populated with NULL. For ECDSA, the parameters field is absent.

\subsubsection{Subject Public Key Algorithm}

\begin{tabular}{|c|c|}
\hline Test Assertion & TA-09.03.02.02 \\
\hline Purpose & $\begin{array}{l}\text { Verifies that the public key algorithm used for generating the keys } \\
\text { is as specified in Table 3-4 of [SP800-78]. }\end{array}$ \\
\hline DTR(s) & $\begin{array}{l}\text { - } \quad \text { DTR-07.03.02.03 } \\
\text { - } \quad \text { DTR-07.03.02.04 }\end{array}$ \\
\hline Issuer Documentation & None. \\
\hline Precondition(s) & $\begin{array}{l}\text { - A token with the Derived PIV Application is inserted into an } \\
\text { appropriate token reader. } \\
\text { - Suitable drivers have been loaded between the test system and } \\
\text { an instance of the reader. } \\
\text { - A digital signature certificate is present within the Derived PIV } \\
\text { Application. }\end{array}$ \\
\hline Test Scenario & 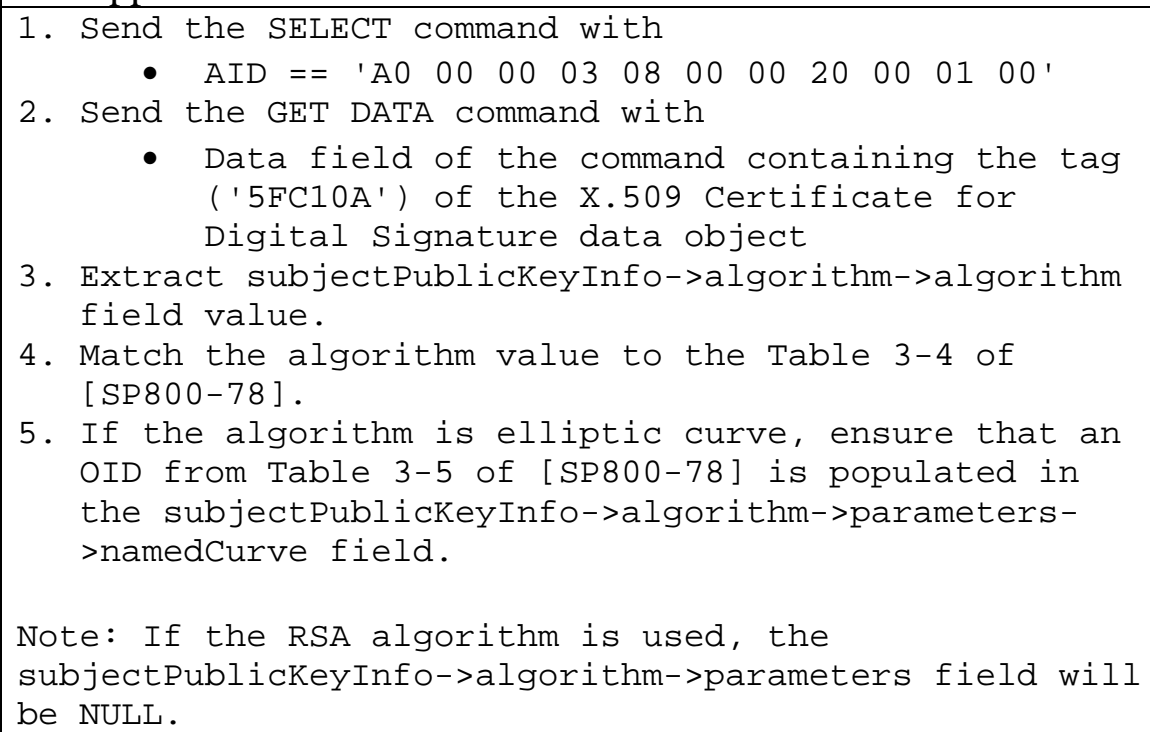 \\
\hline Expected Result(s) & $\begin{array}{l}\text { 1. From Step 1, the command returns the application property } \\
\text { template with the status word ' } 9000 \text { '. } \\
\text { 2. From Step 2, the command returns the requested data object } \\
\text { along with the status word ' } 9000 \text { '. } \\
\text { 3. From Steps } 4 \text { and 5, the digital signature key is generated using } \\
\text { an allowed asymmetric key algorithm. }\end{array}$ \\
\hline
\end{tabular}




\subsubsection{Public Key Size}

\begin{tabular}{|c|c|}
\hline Test Assertion & TA-09.03.02.03 \\
\hline Purpose & $\begin{array}{l}\text { Verifies that the key size requirements are in accordance with } \\
\text { Table 3-1 of [SP800-78]. }\end{array}$ \\
\hline DTR(s) & - $\quad$ DTR-07.03.02.09 \\
\hline Issuer Documentation & None. \\
\hline Precondition(s) & $\begin{array}{l}\text { - A token with the Derived PIV Application is inserted into an } \\
\text { appropriate token reader. } \\
\text { - Suitable drivers have been loaded between the test system and } \\
\text { an instance of the reader. } \\
\text { - A digital signature certificate is present within the Derived PIV } \\
\text { Application. }\end{array}$ \\
\hline Test Scenario & 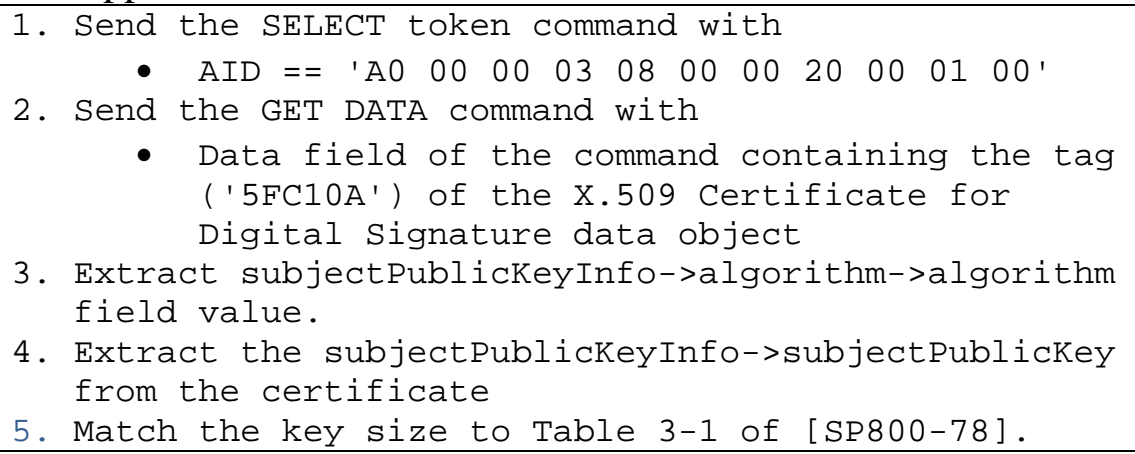 \\
\hline Expected Result(s) & $\begin{array}{l}\text { 1. From Step 1, the command returns the application property } \\
\text { template with the status word ' } 9000 \text { '. } \\
\text { 2. From Step 2, the command returns the requested data object } \\
\text { along with the status word ' } 9000 \text { '. } \\
\text { 3. From Step 5, the key size is in accordance with Table 3-1 of } \\
\text { [SP800-78]. }\end{array}$ \\
\hline
\end{tabular}

\subsubsection{Key Usage Extension}

\begin{tabular}{|c|c|}
\hline Test Assertion & TA-09.03.02.04 \\
\hline Purpose & $\begin{array}{l}\text { Verifies that the digital signature certificate asserts the appropriate } \\
\text { purposes for the key. }\end{array}$ \\
\hline DTR(s) & - $\quad$ DTR-07.03.02.05 \\
\hline Issuer Documentation & None. \\
\hline Precondition(s) & $\begin{array}{l}\text { - A token with the Derived PIV Application is inserted into an } \\
\text { appropriate token reader. } \\
\text { - Suitable drivers have been loaded between the test system and } \\
\text { an instance of the reader. } \\
\text { - A digital signature certificate is present within the Derived PIV } \\
\text { Application. }\end{array}$ \\
\hline Test Scenario & 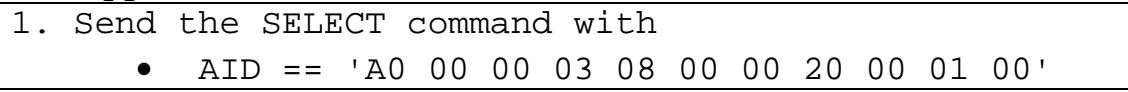 \\
\hline
\end{tabular}




\begin{tabular}{|l|l|}
\hline & $\begin{array}{r}\text { 2. Send the GET DATA command with } \\
\text { thata field of the command containing the tag } \\
\text { ('5FC10A') of the X.509 certificate for } \\
\text { Digital Signature data object }\end{array}$ \\
\hline Expected Result(s) & $\begin{array}{l}\text { Extract the value of the keyUsage extension from } \\
\text { the certificate. }\end{array}$ \\
1. From Step 1, the command returns the application property \\
template with the status word '90 00'. \\
2. From Step 2, the command returns the requested data object \\
along with the status word '90 00'. \\
3rom Step 3, the digitalSignature and nonRepudiation bits have \\
been set. No other bits have been set.
\end{tabular}

\subsubsection{Certificate Policy}

\begin{tabular}{|c|c|}
\hline Test Assertion & TA-09.03.02.05 \\
\hline Purpose & $\begin{array}{l}\text { Verifies that the digital signature certificate asserts the appropriate } \\
\text { certificate policy OID. }\end{array}$ \\
\hline DTR(s) & - $\quad$ DTR-07.03.02.06 \\
\hline Issuer Documentation & None. \\
\hline Precondition(s) & $\begin{array}{l}\text { - A token with the Derived PIV Application is inserted into an } \\
\text { appropriate token reader. } \\
\text { - Suitable drivers have been loaded between the test system and } \\
\text { an instance of the reader. } \\
\text { - A digital signature certificate is present within the Derived PIV } \\
\text { Application. }\end{array}$ \\
\hline Test Scenario & 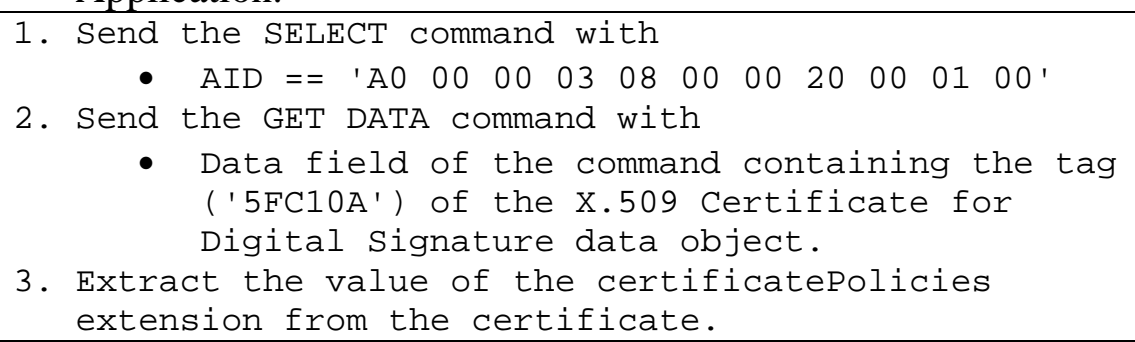 \\
\hline Expected Result(s) & $\begin{array}{l}\text { 1. From Step 1, the command returns the application property } \\
\text { template with the status word ' } 9000 \text { '. } \\
\text { 2. From Step 2, the command returns the requested data object } \\
\text { along with the status word ' } 9000 \text { '. } \\
\text { 3. From Step 3, the certificatePolicies extension asserts one of the } \\
\text { following: id-fpki-common-policy, id-fpki-common-hardware } \\
\text { or id-fpki-common-High. }\end{array}$ \\
\hline
\end{tabular}

\subsubsection{Authority Information Access Extension}

\begin{tabular}{|l|l|}
\hline Test Assertion & TA-09.03.02.06 \\
\hline Purpose & $\begin{array}{l}\text { Verifies that the authority information access extension in the } \\
\text { digital signature certificate is populated appropriately and contains }\end{array}$ \\
\hline
\end{tabular}




\begin{tabular}{|c|c|}
\hline & $\begin{array}{l}\text { an id-ad-caIssuers (1.3.6.1.5.5.7.48.2) accessMethod, which points } \\
\text { to the location where the certificates issued to the issuer of this } \\
\text { certificate can be found. }\end{array}$ \\
\hline DTR(s) & - $\quad$ DTR-07.03.02.07 \\
\hline Issuer Documentation & None. \\
\hline Precondition(s) & $\begin{array}{l}\text { - A token with the Derived PIV Application is inserted into an } \\
\text { appropriate token reader. } \\
\text { - Suitable drivers have been loaded between the test system and } \\
\text { an instance of the reader. } \\
\text { - A digital signature certificate is present within the Derived PIV } \\
\text { Application. }\end{array}$ \\
\hline Test Scenario & 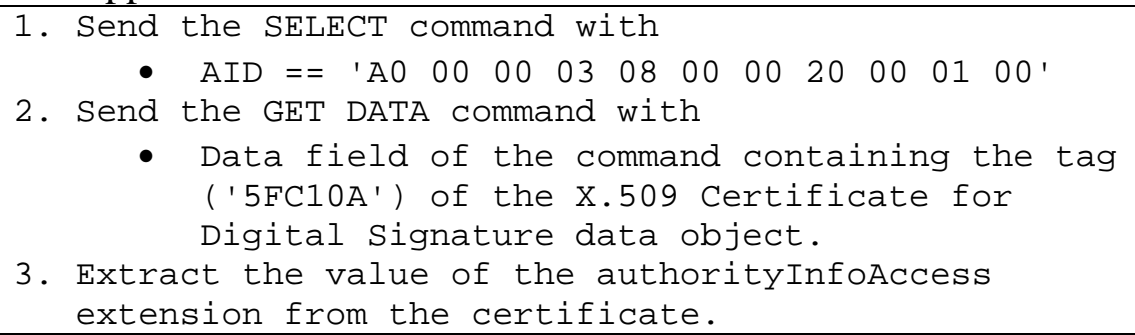 \\
\hline Expected Result(s) & $\begin{array}{l}\text { 1. From Step 1, the command returns the application property } \\
\text { template with the status word ' } 9000 \text { '. } \\
\text { 2. From Step 2, the command returns the requested data object } \\
\text { along with the status word ' } 9000 \text { '. } \\
\text { 3. From Step 3, the authorityInfoAccess extension contains an id- } \\
\text { ad-caIssuers (1.3.6.1.5.5.7.48.2) accessMethod with an } \\
\text { accessLocation of type uniformResourceIdentifier where the } \\
\text { scheme is "http” or "ldap." }\end{array}$ \\
\hline
\end{tabular}

\subsubsection{Asymmetric Key Pair}

\begin{tabular}{|l|l|}
\hline Test Assertion & TA-09.03.02.07 \\
\hline Purpose & $\begin{array}{l}\text { Verifies that the public key that exists in the digital signature } \\
\text { certificate corresponds to the private key within the Derived PIV } \\
\text { Application. }\end{array}$ \\
\hline DTR(s) & - DTR-07.03.02.10 \\
\hline Issuer Documentation & None. \\
\hline Precondition(s) & $\begin{array}{l}\text { - A token with the Derived PIV Application is inserted into an } \\
\text { appropriate token reader. }\end{array}$ \\
& $\begin{array}{l}\text { Suitable drivers have been loaded between the test system and } \\
\text { an instance of the reader. } \\
\end{array}$ \\
- A digital signature certificate is present within the Derived PIV \\
- Application. \\
- The Derived PIV Application Password is recorded. \\
- The Derived PIV Application Password's retry counter is not 0.
\end{tabular}




\begin{tabular}{|c|c|}
\hline Test Scenario & 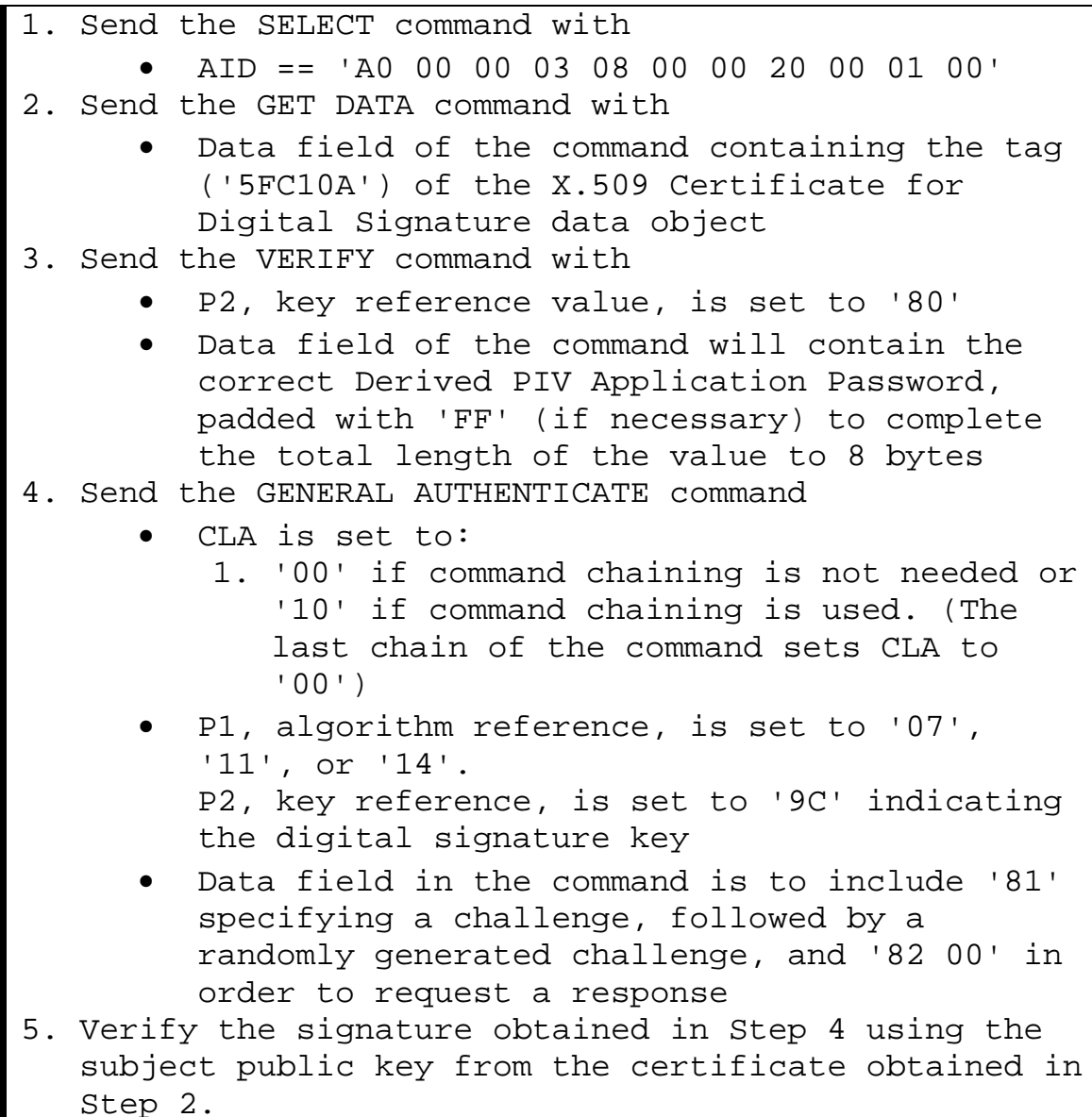 \\
\hline Expected Result(s) & $\begin{array}{l}\text { 1. From Step 1, the command returns the application property } \\
\text { template with the status word ' } 9000 \text { '. } \\
\text { 2. From Step 2, the command returns the requested data object } \\
\text { along with status word ' } 9000 \text { '. } \\
\text { 3. From Step 3, the command returns status word ' } 9000 \text { '. } \\
\text { 4. From Step 4, the command returns the signed challenge with } \\
\text { the status word '90 00' } \\
\text { 5. From Step 5, the private key corresponds to the public key } \\
\text { contained in the certificate as the signature verification } \\
\text { succeeds. }\end{array}$ \\
\hline
\end{tabular}

\subsubsection{8 cRLDistributionPoints Extension}

\begin{tabular}{|l|l|}
\hline Test Assertion & TA-09.03.02.08 \\
\hline Purpose & $\begin{array}{l}\text { Verifies that the cRLDistributionPoints extension in the digital } \\
\text { signature certificate contains at least one URI, either LDAP or } \\
\text { HTTP. }\end{array}$ \\
\hline DTR(s) & $\bullet \quad$ DTR-07.03.02.08 \\
\hline Issuer Documentation & None. \\
\hline
\end{tabular}




\begin{tabular}{|c|c|}
\hline Precondition(s) & $\begin{array}{l}\text { - A token with the Derived PIV Application is inserted into an } \\
\text { appropriate token reader. } \\
\text { - Suitable drivers have been loaded between the test system and } \\
\text { an instance of the reader. } \\
\text { - A digital signature certificate is present within the Derived PIV } \\
\text { Application. }\end{array}$ \\
\hline Test Scenario & 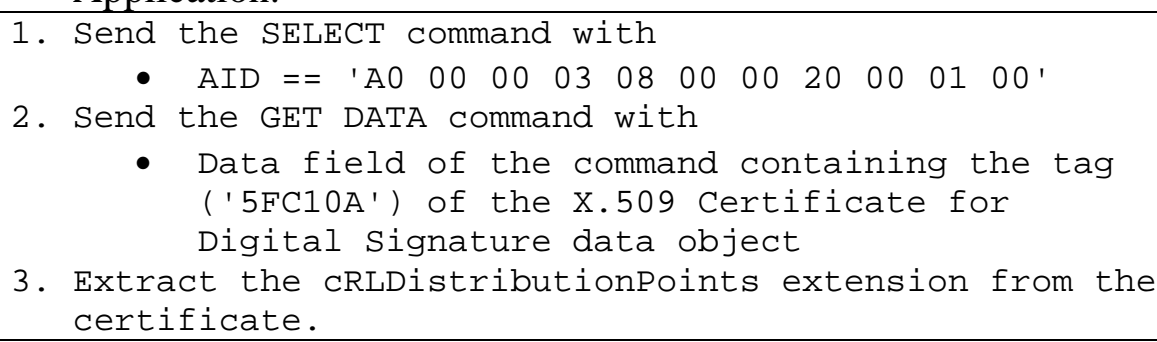 \\
\hline Expected Result(s) & $\begin{array}{l}\text { 1. From Step 1, the command returns the application property } \\
\text { template with the status word ' } 9000 \text { '. } \\
\text { 2. From Step 2, the command returns the requested data object } \\
\text { along with the status word ' } 9000 \text { '. } \\
\text { 3. From Step 3, a URI with either the "LDAP” or "HTTP” scheme } \\
\text { that can be used to access CRL information is present. }\end{array}$ \\
\hline
\end{tabular}

\subsubsection{RSA Exponent}

\begin{tabular}{|c|c|}
\hline Test Assertion & TA-09.03.02.09 \\
\hline Purpose & $\begin{array}{l}\text { Verifies that for RSA keys, the exponent of the asymmetric key for } \\
\text { digital signature is equal to } 65537 .\end{array}$ \\
\hline DTR(s) & - $\quad$ DTR-07.03.02.11 \\
\hline Issuer Documentation & None. \\
\hline Precondition(s) & $\begin{array}{l}\text { - A token with the Derived PIV Application is inserted into an } \\
\text { appropriate token reader. } \\
\text { - Suitable drivers have been loaded between the test system and } \\
\text { an instance of the reader. } \\
\text { - A digital signature certificate is present within the Derived PIV } \\
\text { Application. }\end{array}$ \\
\hline Test Scenario & 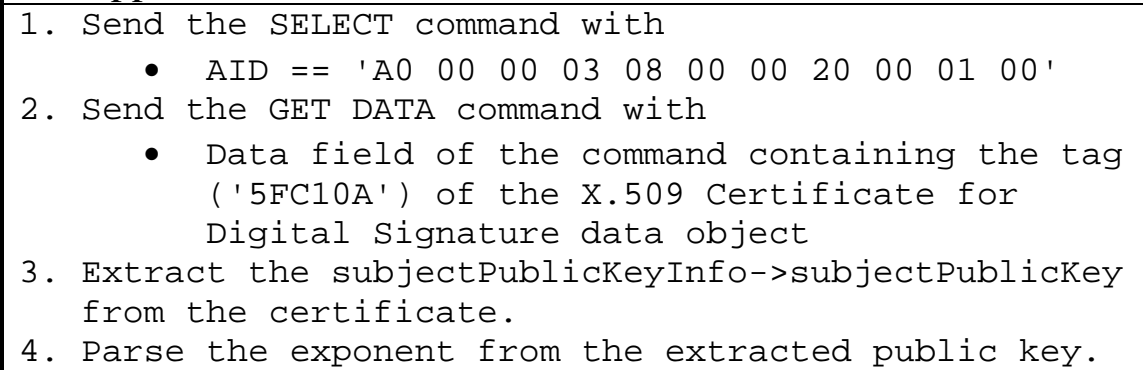 \\
\hline Expected Result(s) & $\begin{array}{l}\text { 1. From Step 1, the command returns the application property } \\
\text { template with the status word ' } 9000 \text { '. }\end{array}$ \\
\hline
\end{tabular}


2. From Step 2, the command returns the requested data object along with the status word ' 90 00'.

3. From Step 4, the exponent of the RSA asymmetric key for digital signature is equal to 65537.

\subsubsection{X.509 Certificate for Key Management ${ }^{20}$}

\subsubsection{Signature Algorithm}

\begin{tabular}{|c|c|}
\hline Test Assertion & TA-09.09.03.01 \\
\hline Purpose & $\begin{array}{l}\text { Verifies that the proper signature algorithm has been used to sign } \\
\text { the key management certificate as specified in Table 3-3 of } \\
\text { [SP800-78]. }\end{array}$ \\
\hline $\operatorname{DTR}(\mathrm{s})$ & $\begin{array}{ll}\text { - } & \text { DTR-07.03.03.01 } \\
\text { - } & \text { DTR-07.03.03.02 } \\
\end{array}$ \\
\hline Issuer Documentation & None. \\
\hline Precondition(s) & $\begin{array}{l}\text { - A token with the Derived PIV Application is inserted into an } \\
\text { appropriate token reader. } \\
\text { - Suitable drivers have been loaded between the test system and } \\
\text { an instance of the reader. } \\
\text { - A key management certificate is present within the Derived } \\
\text { PIV Application. }\end{array}$ \\
\hline Test Scenario & 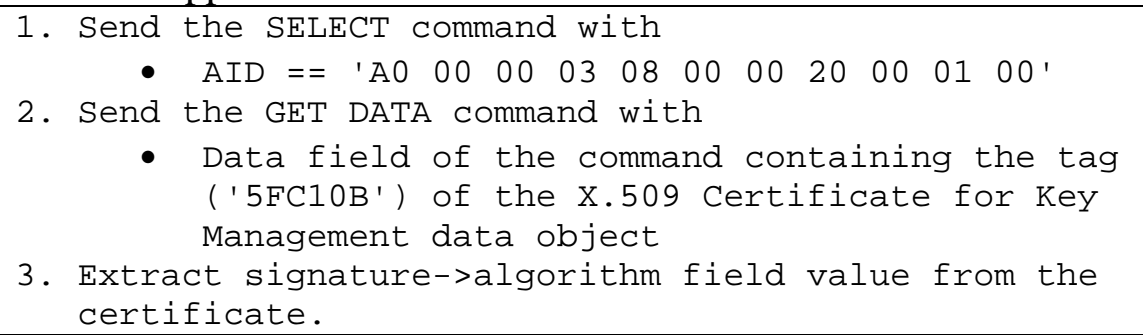 \\
\hline Expected Result(s) & $\begin{array}{l}\text { 1. From Step 1, the command returns the application property } \\
\text { template with the status word ' } 9000 \text { '. } \\
\text { 2. From Step 2, the command returns the requested data object } \\
\text { along with the status word ' } 9000 \text { '. } \\
\text { 3. From Step 3, the algorithm value is in accordance with Table 3- } \\
3 \text { of [SP800-78]. If the algorithm value is id-RSASSA-PSS, } \\
\text { then the hashAlgorithm field in signature->parameters field is } \\
\text { populated with SHA-256 (OID = 2.16.840.1.101.3.4.2.1). For } \\
\text { RSA with PKCS \#1 v1.5 padding, the parameters field is } \\
\text { populated with NULL. For ECDSA, the parameters field is } \\
\text { absent. }\end{array}$ \\
\hline
\end{tabular}

\footnotetext{
${ }^{20}$ The key management key and certificate may be tested outside of the Derived PIV Application. Specific test assertions can be developed by test entities to test this key and certificate based on the environment (e.g., email application) in which the key pair is being used. See Appendix A for example of testing approaches.
} 


\subsubsection{Subject Public Key Algorithm}

\begin{tabular}{|c|c|}
\hline Test Assertion & TA-09.03.03.02 \\
\hline Purpose & $\begin{array}{l}\text { Verifies that the public key algorithm used for generating the keys is } \\
\text { as specified in Table 3-4 of [SP800-78]. }\end{array}$ \\
\hline DTR(s) & $\begin{array}{l}-\quad \text { DTR-07.03.03.03 } \\
\text { - } \quad \text { DTR-07.03.03.04 }\end{array}$ \\
\hline Issuer Documentation & None. \\
\hline Precondition(s) & $\begin{array}{l}\text { - A token with the Derived PIV Application is inserted into an } \\
\text { appropriate token reader. } \\
\text { - Suitable drivers have been loaded between the test system and } \\
\text { an instance of the reader. } \\
\text { - A key management certificate is present within the Derived PIV } \\
\text { Application. }\end{array}$ \\
\hline Test Scenario & 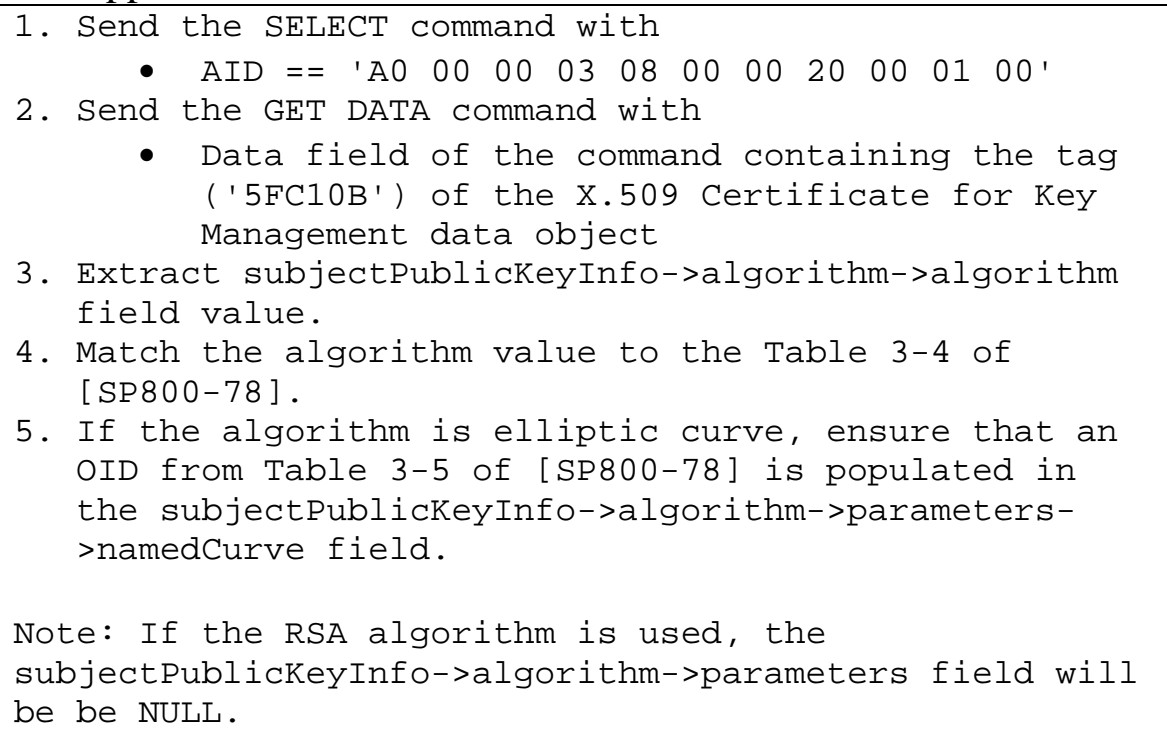 \\
\hline Expected Result(s) & $\begin{array}{l}\text { 1. From Step 1, the command returns the application property } \\
\text { template with the status word ' } 9000 \text { '. } \\
\text { 2. From Step 2, the command returns the requested data object } \\
\text { along with the status word ' } 9000 \text { '. } \\
\text { 3. From Steps } 4 \text { and 5, the key management key is generated using } \\
\text { an allowed asymmetric key algorithm. }\end{array}$ \\
\hline
\end{tabular}

\subsubsection{Public Key Size}

\begin{tabular}{|l|l|}
\hline Test Assertion & TA-09.09.03.03 \\
\hline Purpose & $\begin{array}{l}\text { Verifies that the key size requirements are in accordance with } \\
\text { Table 3-1 of [SP800-78]. }\end{array}$ \\
\hline DTR(s) & $\bullet \quad$ DTR-07.03.03.09 \\
\hline Issuer Documentation & None. \\
\hline
\end{tabular}




\begin{tabular}{|c|c|}
\hline Precondition(s) & $\begin{array}{l}\text { - A token with the Derived PIV Application is inserted into an } \\
\text { appropriate token reader. } \\
\text { - Suitable drivers have been loaded between the test system and } \\
\text { an instance of the reader. } \\
\text { - A key management certificate is present within the Derived } \\
\text { PIV Application. }\end{array}$ \\
\hline Test Scenario & 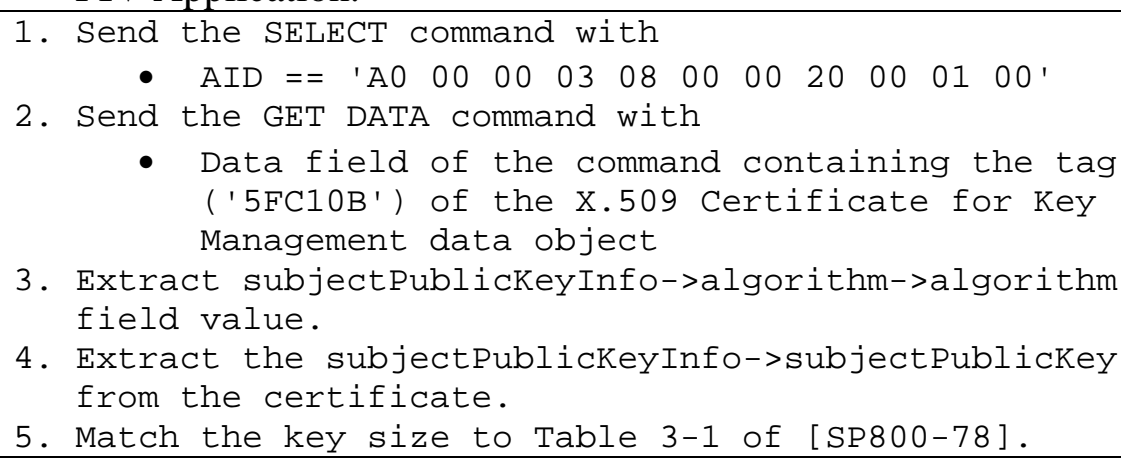 \\
\hline Expected Result(s) & $\begin{array}{l}\text { 1. From Step 1, the command returns the application property } \\
\text { template with the status word ' } 9000 \text { '. } \\
\text { 2. From Step 2, the command returns the requested data object } \\
\text { along with the status word ' } 9000 \text { '. } \\
\text { 3. From Step 5, the key size is in accordance with Table 3-1 of } \\
\text { [SP800-78]. }\end{array}$ \\
\hline
\end{tabular}

\subsubsection{Key Usage Extension}

\begin{tabular}{|c|c|}
\hline Test Assertion & TA-09.03.03.04 \\
\hline Purpose & $\begin{array}{l}\text { Verifies the key management certificate asserts the appropriate } \\
\text { purposes for the key. }\end{array}$ \\
\hline DTR(s) & - DTR-07.03.03.05 \\
\hline Issuer Documentation & None. \\
\hline Precondition(s) & $\begin{array}{l}\text { - A Token with the Derived PIV Application is inserted into an } \\
\text { appropriate token reader. } \\
\text { - Suitable drivers have been loaded between the test system and } \\
\text { an instance of the reader. } \\
\text { - A key management certificate is present within the Derived } \\
\text { PIV Application. }\end{array}$ \\
\hline Test Scenario & 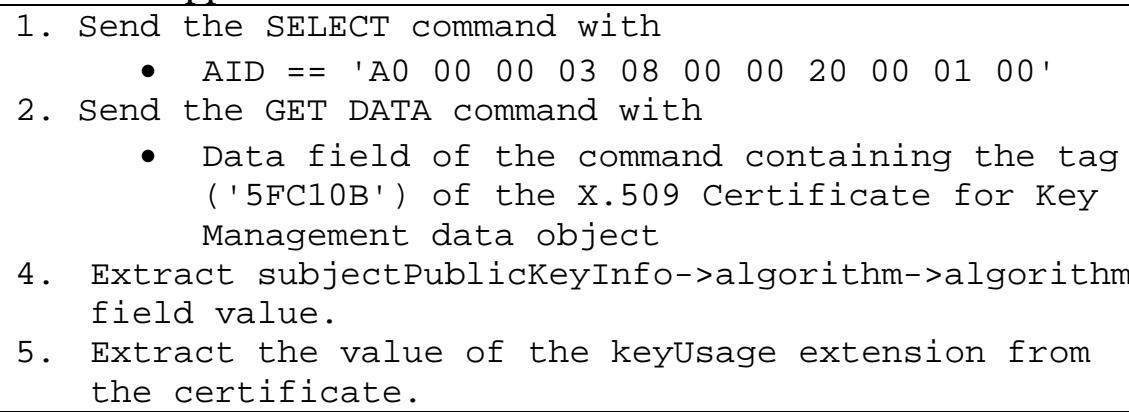 \\
\hline
\end{tabular}


Expected Result(s)

1. From Step 1, the command returns the application property template with the status word '90 00'.

2. From Step 2, the command returns the requested data object along with the status word '90 00'.

3. From Step 5, if the public key algorithm is RSA, then the keyUsage extension only asserts the keyEncipherment bit. If the public key algorithm is elliptic curve, then the keyUsage extension only asserts the keyAgreement bit.

\subsubsection{Certificate Policy}

\begin{tabular}{|c|c|}
\hline Test Assertion & TA-09.03.03.05 \\
\hline Purpose & $\begin{array}{l}\text { Verifies the key management certificate asserts the appropriate } \\
\text { certificate policy OID. }\end{array}$ \\
\hline DTR(s) & - $\quad$ DTR-07.03.03.06 \\
\hline Issuer Documentation & None. \\
\hline Precondition(s) & $\begin{array}{l}\text { - A token with the Derived PIV Application is inserted into an } \\
\text { appropriate token reader. } \\
\text { - Suitable drivers have been loaded between the test system and } \\
\text { an instance of the reader. } \\
\text { - A key management certificate is present within the Derived } \\
\text { PIV Application. }\end{array}$ \\
\hline Test Scenario & 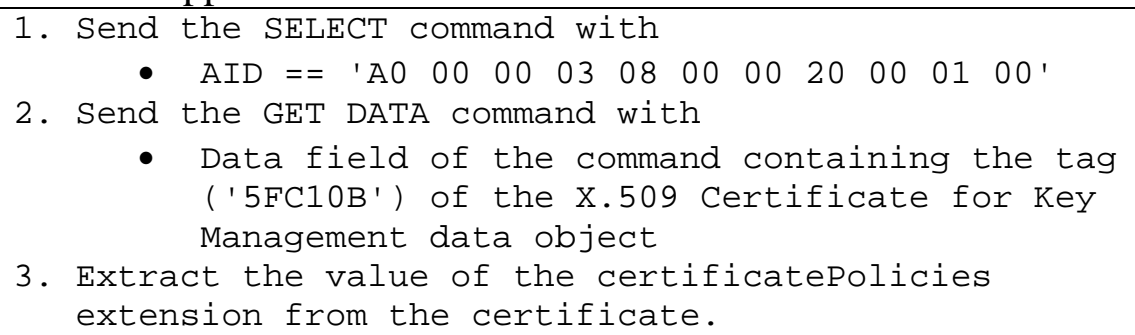 \\
\hline Expected Result(s) & $\begin{array}{l}\text { 1. From Step 1, the command returns the application property } \\
\text { template with the status word ' } 9000 \text { '. } \\
\text { 2. From Step 2, the command returns the requested data object } \\
\text { along with the status word ' } 9000 \text { '. } \\
\text { 3. From Step 3, the certificatePolicies extension asserts one of the } \\
\text { following: id-fpki-common-policy, id-fpki-common-hardware } \\
\text { or id-fpki-common-High. }\end{array}$ \\
\hline
\end{tabular}

\subsubsection{Authority Information Access Extension}

\begin{tabular}{|l|l|}
\hline Test Assertion & TA-09.03.03.06 \\
\hline Purpose & $\begin{array}{l}\text { Verifies that the authority information access extension in the key } \\
\text { management certificate is populated appropriately and contains an } \\
\text { id-ad-caIssuers (1.3.6.1.5.5.7.48.2) accessMethod, which points to } \\
\text { the location where the certificates issued to the issuer of this } \\
\text { certificate can be found. }\end{array}$ \\
\hline
\end{tabular}




\begin{tabular}{|c|c|}
\hline DTR(s) & - $\quad$ DTR-07.03.03.07 \\
\hline Issuer Documentation & None. \\
\hline Precondition(s) & $\begin{array}{l}\text { - A token with the Derived PIV Application is inserted into an } \\
\text { appropriate token reader. } \\
\text { - Suitable drivers have been loaded between the test system and } \\
\text { an instance of the reader. } \\
\text { - A key management certificate is present within the Derived } \\
\text { PIV Application. }\end{array}$ \\
\hline Test Scenario & 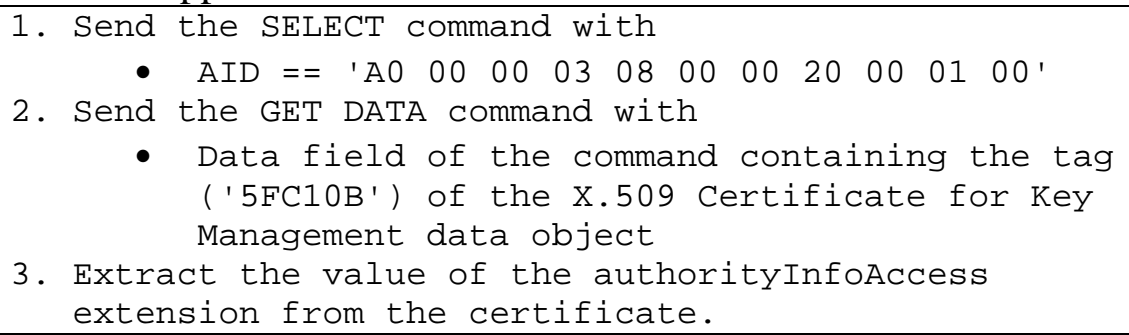 \\
\hline Expected Result(s) & $\begin{array}{l}\text { 1. From Step 1, the command returns the application property } \\
\text { template with the status word ' } 9000 \text { '. } \\
\text { 2. From Step 2, the command returns the requested data object } \\
\text { along with the status word ' } 9000 \text { '. } \\
\text { 3. From Step 3, the authorityInfoAccess extension contains an id- } \\
\text { ad-caIssuers (1.3.6.1.5.5.7.48.2) accessMethod with an } \\
\text { accessLocation of type uniformResourceIdentifier where the } \\
\text { scheme is "http" or "ldap." }\end{array}$ \\
\hline
\end{tabular}

\subsubsection{Asymmetric Key Pair}

\begin{tabular}{|c|c|}
\hline Test Assertion & TA-09.03.03.07 \\
\hline Purpose & $\begin{array}{l}\text { Verifies that the public key that exists in the key management } \\
\text { certificate corresponds to the private key within the Derived PIV } \\
\text { Application. }\end{array}$ \\
\hline DTR(s) & - DTR-07.03.03.10 \\
\hline Issuer Documentation & None. \\
\hline Precondition(s) & $\begin{array}{l}\text { - A Token with the Derived PIV Application is inserted into an } \\
\text { appropriate token reader. } \\
\text { - Suitable drivers have been loaded between the test system and } \\
\text { an instance of the reader. } \\
\text { - A key management certificate is present within the Derived } \\
\text { PIV Application. } \\
\text { - The Derived PIV Application Password is recorded. } \\
\text { - The Derived PIV Application Password's retry counter is not } 0 .\end{array}$ \\
\hline Test Scenario & 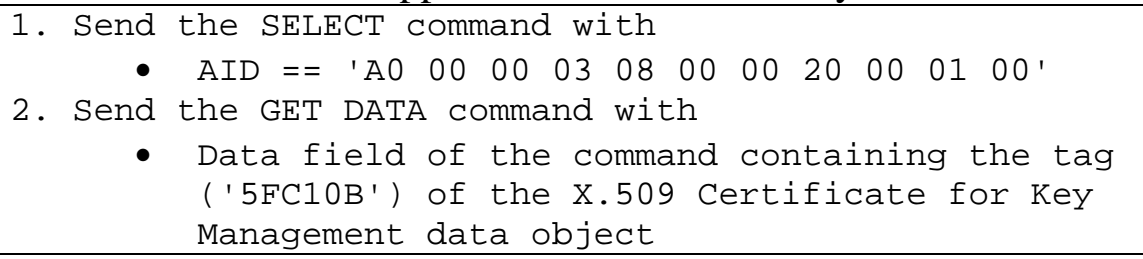 \\
\hline
\end{tabular}




\begin{tabular}{|c|c|}
\hline & 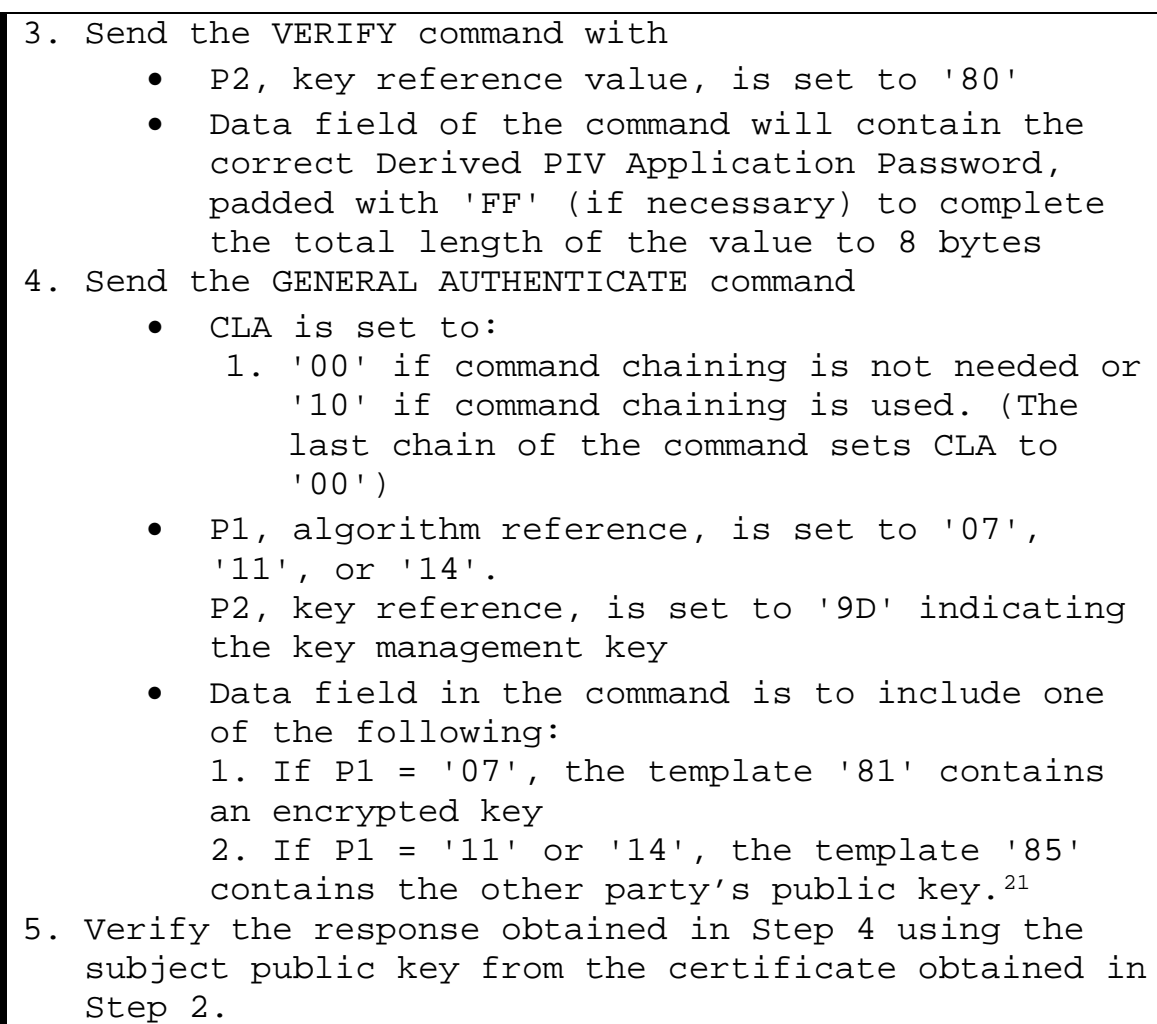 \\
\hline Expected Result(s) & $\begin{array}{l}\text { 1. From Step 1, the command returns the application property } \\
\text { template with the status word ' } 9000 \text { '. } \\
\text { 2. From Step 2, the command returns the requested data object } \\
\text { along with the status word ' } 9000 \text { '. } \\
\text { 3. From Step 3, the command returns status word ' } 9000 \text { ' } \\
\text { 4. From Step 4, for algorithm reference '07', the command returns } \\
\text { the transported key with status word ' } 9000 \text { '. For algorithm } \\
\text { reference ' } 11 \text { ' or ' } 14 \text { ', the command returns the shared secret } Z^{22} \\
\text { with status word ' } 9000 \text { '. } \\
\text { 5. From Step 5, the private key corresponds to the public key } \\
\text { contained in the certificate. For algorithm reference '07', the } \\
\text { test tool application's copy of the plaintext key corresponds to } \\
\text { the one received in the response to Step } 4 \text { from the token. For } \\
\text { algorithm reference ' } 11 \text { ' or ' } 14 \text { ', the shared secret returned in } \\
\text { Step } 4 \text { matches the shared secret computed off token. }\end{array}$ \\
\hline
\end{tabular}

\footnotetext{
${ }^{21}$ Template '85' contains the other party's public key, a point on Curve P-256 or P-384, encoded as '04' || X \| Y, without the use of point compression, as described in Section 2.3.3 of [SEC1].

${ }^{22} \mathrm{Z}$ is the $\mathrm{X}$ coordinate of point $\mathrm{P}$ as defined in [SP800-56A], Section 5.7.1.2
} 


\subsubsection{8 cRLDistributionPoints Extension}

\begin{tabular}{|c|c|}
\hline Test Assertion & TA-09.03.03.08 \\
\hline Purpose & $\begin{array}{l}\text { Verifies that the cRLDistributionPoints extension in the key } \\
\text { management certificate contains at least one URIs, either LDAP or } \\
\text { HTTP. }\end{array}$ \\
\hline DTR(s) & • $\quad$ DTR-07.03.03.08 \\
\hline Issuer Documentation & None. \\
\hline Precondition(s) & $\begin{array}{l}\text { - A token with the Derived PIV Application is inserted into an } \\
\text { appropriate token reader. } \\
\text { - Suitable drivers have been loaded between the test system and } \\
\text { an instance of the reader. } \\
\text { - A key management certificate is present within the Derived PIV } \\
\text { Application. }\end{array}$ \\
\hline Test Scenario & 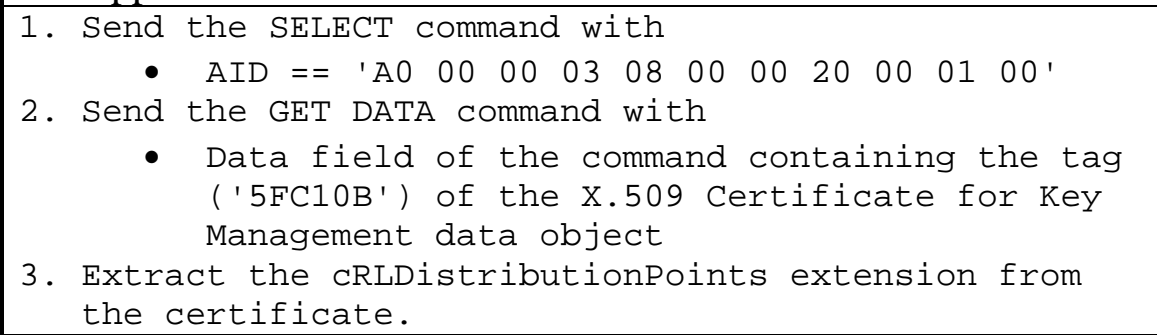 \\
\hline Expected Result(s) & $\begin{array}{l}\text { 1. From Step 1, the command returns the application property } \\
\text { template with the status word ' } 9000 \text { '. } \\
\text { 2. From Step 2, the command returns the requested data object } \\
\text { along with the status word ' } 9000 \text { '. } \\
\text { 3. From Step 3, a URI with either the "LDAP” or "HTTP” scheme } \\
\text { that can be used to access CRL information is present. }\end{array}$ \\
\hline
\end{tabular}

\subsubsection{RSA Exponent}

\begin{tabular}{|c|c|}
\hline Test Assertion & TA-09.09.03.09 \\
\hline Purpose & $\begin{array}{l}\text { Verifies that for RSA keys, the exponent of the asymmetric key for } \\
\text { key management is equal to } 65537 \text {. }\end{array}$ \\
\hline DTR(s) & - $\quad$ DTR-07.03.03.11 \\
\hline Issuer Documentation & None. \\
\hline Precondition(s) & $\begin{array}{l}\text { - A token with the Derived PIV Application is inserted into an } \\
\text { appropriate token reader. } \\
\text { - Suitable drivers have been loaded between the test system and } \\
\text { an instance of the reader. } \\
\text { - A key management certificate is present within the Derived } \\
\text { PIV Application. }\end{array}$ \\
\hline Test Scenario & 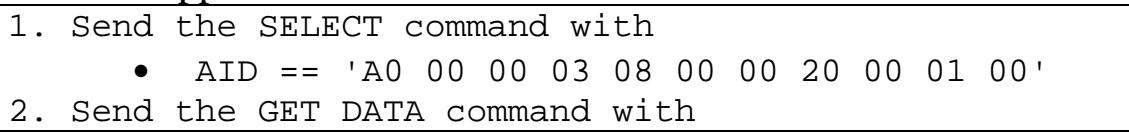 \\
\hline
\end{tabular}




\begin{tabular}{|l|l|}
\hline $\begin{array}{r}\text { Data field of the command containing the tag } \\
(\text { '5FC1OB') of the X.509 Certificate for Key } \\
\text { Management data object }\end{array}$ & $\begin{array}{l}\text { 3. Extract the subjectPublickeyInfo->subjectPublickey } \\
\text { from the certificate. } \\
\text { 4. Parse the exponent from the extracted public key. }\end{array}$ \\
\hline Expected Result(s) & $\begin{array}{l}\text { 1. From Step 1, the command returns the application property } \\
\text { template with the status word '90 00'. }\end{array}$ \\
2. From Step 2, the command returns the requested data object \\
along with the status word '90 00'. \\
3rom Step 4, the exponent of the RSA asymmetric key for key \\
management is equal to 65 537.
\end{tabular}

\subsubsection{X.509 Certificate of the Derived PIV Credential Issuer (Content Signing)}

\subsubsection{Signature Algorithm}

\begin{tabular}{|c|c|}
\hline Test Assertion & TA-09.03.04.01 \\
\hline Purpose & $\begin{array}{l}\text { Verifies that the signature field of the Derived PIV Credential } \\
\text { Issuer's (content signing) certificate specifies one of the following } \\
\text { algorithm OIDs: } 1.2 .840 .113549 .1 .10 \text { (id-RSASSA-PSS), } \\
\text { 1.2.840.113549.1.11 (Sha256WithRSAEncryption), } \\
\text { 1.2.840.10045.4.3.2 (edsa-with-Sha256), 1.2.840.10045.4.3.3 } \\
\text { (edsa-with-Sha384). }\end{array}$ \\
\hline DTR(s) & $\begin{array}{ll}- & \text { DTR-07.03.04.01 } \\
- & \text { DTR-07.03.04.02 }\end{array}$ \\
\hline Issuer Documentation & None. \\
\hline Precondition(s) & $\begin{array}{l}\text { - A token with the Derived PIV Application is inserted into an } \\
\text { appropriate token reader. } \\
\text { - Suitable drivers have been loaded between the test system and } \\
\text { an instance of the reader. } \\
\text { - A Security Object is present within the Derived PIV } \\
\text { Application. }\end{array}$ \\
\hline Test Scenario & 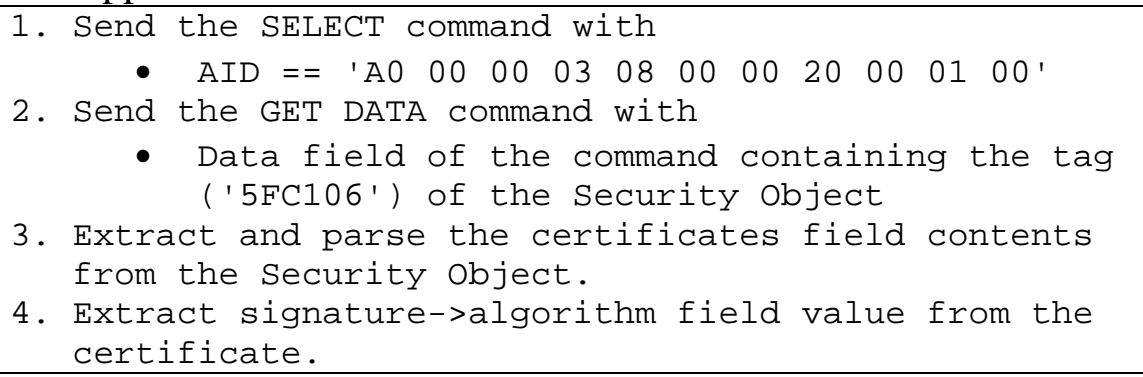 \\
\hline Expected Result(s) & $\begin{array}{l}\text { 1. From Step 1, the command returns the application property } \\
\text { template with the status word ' } 9000 \text { '. } \\
\text { 2. From Step 2, the command returns the requested data object } \\
\text { along with the status word ' } 9000 \text { '. } \\
\text { 3. From Step 4, the algorithm value is in accordance with Table 3- } \\
3 \text { of [SP800-78]. If the algorithm value is id-RSASSA-PSS, }\end{array}$ \\
\hline
\end{tabular}


then the hashAlgorithm field in signature->parameters is populated with SHA-256 (OID $=2 \cdot 16.840 .1 .101 .3 .4 .2 .1)$. For RSA with PKCS \#1 v1.5 padding, the parameters field is populated with NULL. For ECDSA, the parameters field is absent.

\subsubsection{Subject Public Key Algorithm}

\begin{tabular}{|c|c|}
\hline Test Assertion & TA-09.03.04.02 \\
\hline Purpose & $\begin{array}{l}\text { Verifies that the public key algorithm used for generating the keys } \\
\text { is one of the following OIDs: } 1.2 .840 .113549 .1 .1 .1 \text { (RSA } \\
\text { Encryption) or 1.2.840.10045.2.1 (elliptic curve key). }\end{array}$ \\
\hline DTR(s) & $\begin{array}{ll} & \text { DTR-07.03.04.03 } \\
\text { - } & \text { DTR-07.03.04.04 }\end{array}$ \\
\hline Issuer Documentation & None. \\
\hline Precondition(s) & $\begin{array}{l}\text { - A token with the Derived PIV Application is inserted into an } \\
\text { appropriate token reader. } \\
\text { - Suitable drivers have been loaded between the test system and } \\
\text { an instance of the reader. } \\
\text { - A Security Object is present within the Derived PIV } \\
\text { Application. }\end{array}$ \\
\hline Test Scenario & 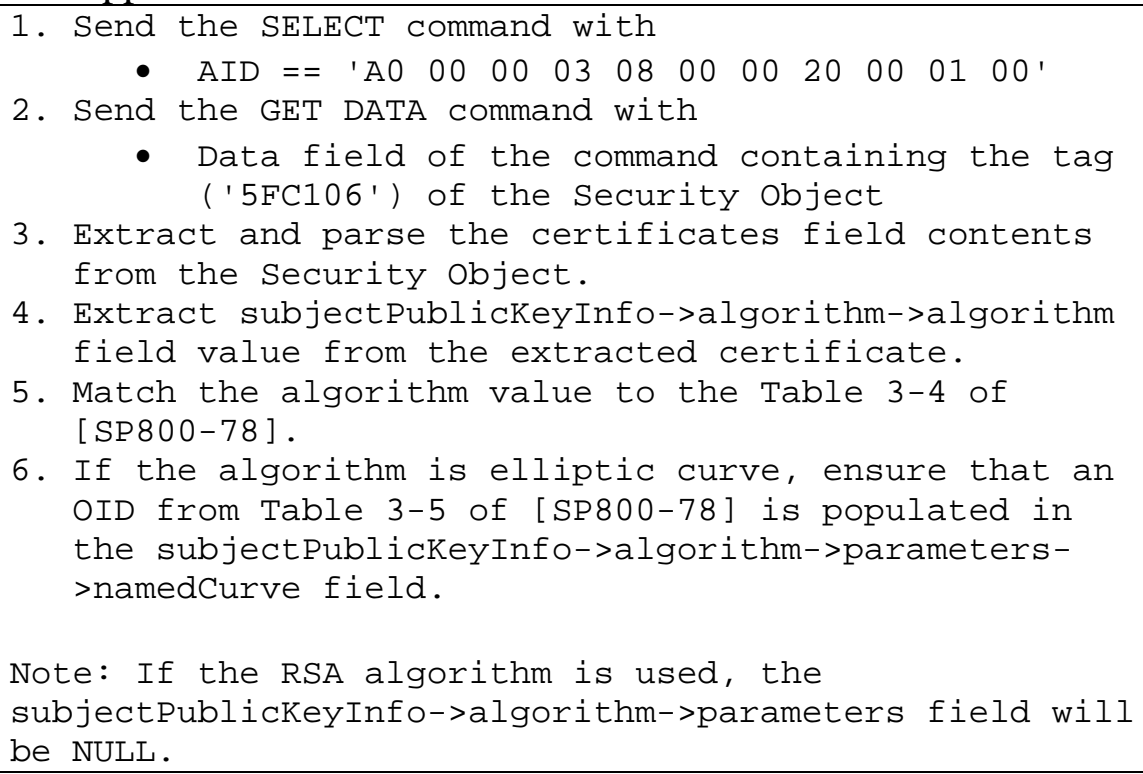 \\
\hline Expected Result(s) & $\begin{array}{l}\text { 1. From Step 1, the command returns the application property } \\
\text { template with the status word ' } 9000 \text { '. } \\
\text { 2. From Step 2, the command returns the requested data object } \\
\text { along with the status word ' } 9000 \text { '. } \\
\text { 3. From Step 5, the Derived PIV Credential Issuer's (content } \\
\text { signing) key pair is generated using an allowed asymmetric key } \\
\text { algorithm. }\end{array}$ \\
\hline
\end{tabular}


4. From Step 6, the Derived PIV Credential Issuer's (content signing) key pair is generated using an allowed curve.

\subsubsection{Public Key Size S23 $^{23}$}

\begin{tabular}{|c|c|}
\hline Test Assertion & TA-09.03.04.03 \\
\hline Purpose & $\begin{array}{l}\text { Verifies that size of the subject public key in the Derived PIV } \\
\text { Credential Issuer's (content signing) certificate conforms to Table } \\
3-2 \text { of [SP800-78]. }\end{array}$ \\
\hline DTR(s) & - DTR-07.03.04.10 \\
\hline Issuer Documentation & None. \\
\hline Precondition(s) & $\begin{array}{l}\text { - A token with the Derived PIV Application is inserted into an } \\
\text { appropriate token reader. } \\
\text { - Suitable drivers have been loaded between the test system and } \\
\text { an instance of the reader. } \\
\text { - A Security Object is present within the Derived PIV } \\
\text { Application. }\end{array}$ \\
\hline Test Scenario & 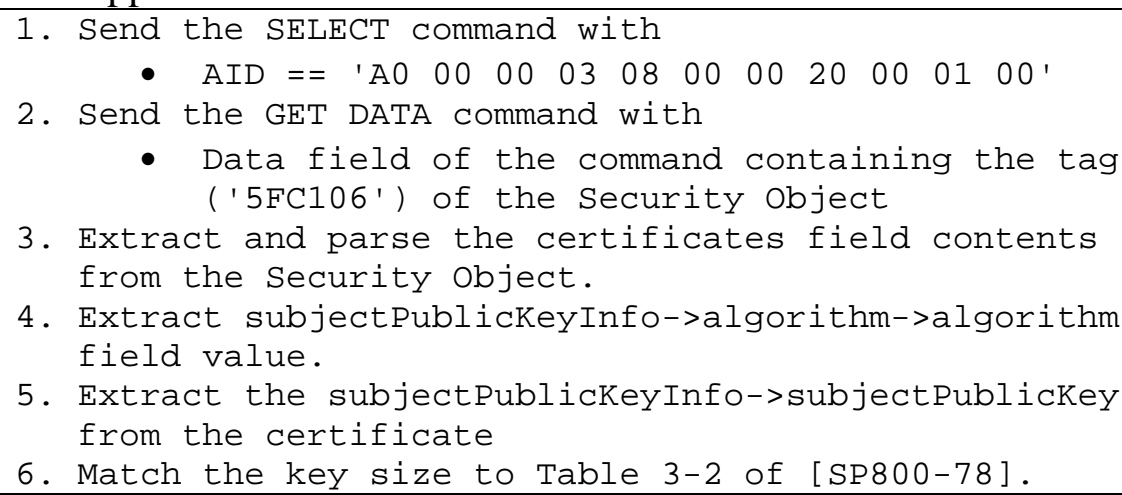 \\
\hline Expected Result(s) & $\begin{array}{l}\text { 1. From Step 1, the command returns the application property } \\
\text { template with the status word ' } 9000 \text { '. } \\
\text { 2. From Step 2, the command returns the requested data object } \\
\text { along with the status word ' } 9000 \text { '. } \\
\text { 3. From Step 6, the key size is in accordance with Table 3-2 of } \\
\text { [SP800-78]. }\end{array}$ \\
\hline
\end{tabular}

\subsubsection{Key Usage Extension}

\begin{tabular}{|l|l|}
\hline Test Assertion & TA-09.03.04.04 \\
\hline Purpose & $\begin{array}{l}\text { Verifies the Derived PIV Credential Issuer's (content signing) } \\
\text { certificate asserts the appropriate purpose for the key. }\end{array}$ \\
\hline DTR(s) & $\bullet \quad$ DTR-07.03.04.05 \\
\hline Issuer Documentation & None. \\
\hline
\end{tabular}

${ }^{23}$ Note that the Security Object for a Derived PIV Application is signed using a private key whose corresponding public key is contained in a Derived PIV Credential Issuer's (content signing) certificate. 


\begin{tabular}{|l|l|}
\hline Precondition(s) & $\begin{array}{l}\text { A token with the Derived PIV Application is inserted into an } \\
\text { appropriate token reader. } \\
\text { - Suitable drivers have been loaded between the test system and } \\
\text { an instance of the reader. } \\
\text { A Security Object is present within the Derived PIV } \\
\text { Application. }\end{array}$ \\
\hline Test Scenario & $\begin{array}{l}\text { 1. Send the SELECT command with } \\
\text { 2. Send the GET DATA command with } \\
\text { Data field of the command containing the tag } \\
\text { ('5FC106') of the Security Object }\end{array}$ \\
$\begin{array}{l}\text { 3. Extract and parse the certificates field contents } \\
\text { from the Security Object. }\end{array}$ \\
$\begin{array}{l}\text { 4. Extract the value of the keyUsage extension from } \\
\text { the certificate. }\end{array}$ \\
\hline Expected Result(s) & $\begin{array}{l}\text { From Step 1, the command returns the application property } \\
\text { template with the status word '90 00'. } \\
\text { From Step 2, the command the requested data object along with } \\
\text { the returns status word '90 00'. }\end{array}$ \\
3. From Step 4, the digitalSignature bit has been set. No other bits \\
have been set.
\end{tabular}

\subsubsection{Certificate Policy}

\begin{tabular}{|c|c|}
\hline Test Assertion & TA-09.03.04.05 \\
\hline Purpose & $\begin{array}{l}\text { Verifies the Derived PIV Credential Issuer's (content signing) } \\
\text { certificate asserts the appropriate certificate policy OID. }\end{array}$ \\
\hline DTR(s) & - $\quad$ DTR-07.03.04.06 \\
\hline Issuer Documentation & None. \\
\hline Precondition(s) & $\begin{array}{l}\text { - A token with the Derived PIV Application is inserted into an } \\
\text { appropriate token reader. } \\
\text { - Suitable drivers have been loaded between the test system and } \\
\text { an instance of the reader. } \\
\text { - A Security Object is present within the Derived PIV } \\
\text { Application. }\end{array}$ \\
\hline Test Scenario & $\begin{array}{l}\text { 1. Send the SELECT command with } \\
\bullet \text { AID == 'A } \odot \odot \odot \odot \odot 3 \odot 8 \odot \odot \odot \odot 2 \odot \odot \odot \odot 1 \odot \odot \text { ' } \\
\text { 2. Send the GET DATA command with } \\
\bullet \text { Data field of the command containing the tag } \\
\text { ('5FC106') of the Security Object } \\
\text { 3. Extract and parse the certificates field contents } \\
\text { from the Security object. } \\
\text { 4. Extract the value of the certificatePolicies } \\
\text { extension from the certificate. }\end{array}$ \\
\hline Expected Result(s) & $\begin{array}{l}\text { 1. From Step 1, the command returns the application property } \\
\text { template with the status word ' } 9000 \text { '. } \\
\text { 2. From Step 2, the command the requested data object along with } \\
\text { the returns status word ' } 9000 \text { '. }\end{array}$ \\
\hline
\end{tabular}


3. From Step 4, the certificatePolicies extension asserts the idfpki-common-piv-contentSigning policy.

\subsubsection{Extended Key Usage}

\begin{tabular}{|c|c|}
\hline Test Assertion & TA-09.03.04.06 \\
\hline Purpose & $\begin{array}{l}\text { Verifies the Derived PIV Credential Issuer's (content signing) } \\
\text { certificate asserts the appropriate OID in the extended key usage } \\
\text { extension. }\end{array}$ \\
\hline DTR(s) & - $\quad$ DTR-07.03.04.07 \\
\hline Issuer Documentation & None. \\
\hline Precondition(s) & $\begin{array}{l}\text { - A token with the Derived PIV Application is inserted into an } \\
\text { appropriate token reader. } \\
\text { - Suitable drivers have been loaded between the test system and } \\
\text { an instance of the reader. } \\
\text { - A Security Object is present within the Derived PIV } \\
\text { Application. }\end{array}$ \\
\hline Test Scenario & $\begin{array}{l}\text { 1. Send the SELECT command with } \\
\bullet \text { AID == 'A } \odot \odot \odot \odot \odot 3 \odot 8 \odot \odot \odot \odot 2 \odot \odot \odot \odot 1 \odot \odot \text { ' } \\
\text { 2. Send the GET DATA command with } \\
\text { - Data field of the command containing the tag } \\
\text { ('5FC106') of the Security Object } \\
\text { 3. Extract and parse the certificates field contents } \\
\text { from the Security object. } \\
\text { 4. Extract the value of the extKeyUsage extension from } \\
\text { the certificate. }\end{array}$ \\
\hline Expected Result(s) & $\begin{array}{l}\text { 1. From Step 1, the command returns the application property } \\
\text { template with the status word ' } 9000 \text { '. } \\
\text { 2. From Step 2, the command returns the requested data object } \\
\text { along with the status word ' } 9000 \text { '. } \\
\text { 3. From Step 4, the extended key usage extension asserts the id- } \\
\text { PIV-content-signing OID, indicating that the certificate is } \\
\text { authorized to sign PIV data objects. }\end{array}$ \\
\hline
\end{tabular}

\subsubsection{Authority Information Access Extension}

\begin{tabular}{|l|l|}
\hline Test Assertion & TA-09.03.04.07 \\
\hline Purpose & $\begin{array}{l}\text { Verifies the authority information access extension in the Derived } \\
\text { PIV Credential Issuer's (content signing) certificate is populated } \\
\text { appropriately and contains the id-ad-caIssuers (1.3.6.1.5.5.7.48.2) } \\
\text { accessMethod, which points to the location where the certificates } \\
\text { issued to the issuer of this certificate can be found. }\end{array}$ \\
\hline DTR(s) & $\bullet \quad$ DTR-07.03.04.08 \\
\hline Issuer Documentation & None. \\
\hline
\end{tabular}




\begin{tabular}{|c|c|}
\hline Precondition(s) & $\begin{array}{l}\text { - A token with the Derived PIV Application is inserted into an } \\
\text { appropriate token reader. } \\
\text { - Suitable drivers have been loaded between the test system and } \\
\text { an instance of the reader. } \\
\text { - A Security Object is present within the Derived PIV } \\
\text { Application. }\end{array}$ \\
\hline Test Scenario & 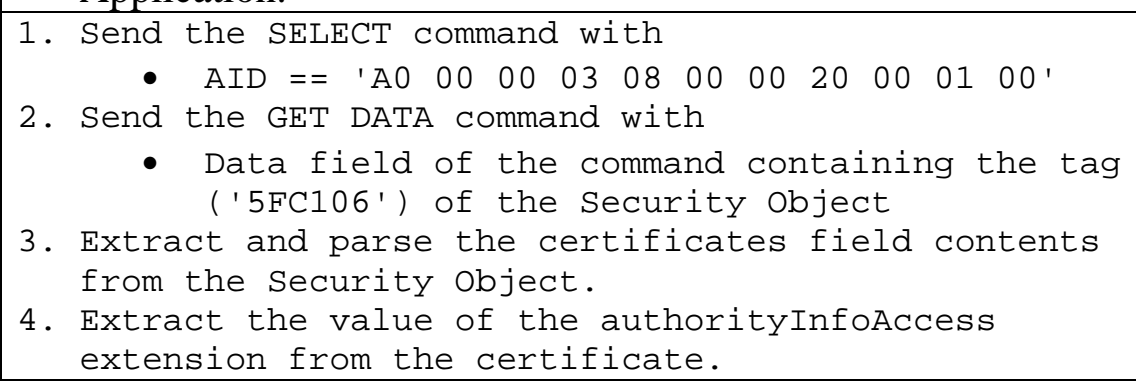 \\
\hline Expected Result(s) & $\begin{array}{l}\text { 1. From Step 1, the command returns the application property } \\
\text { template with the status word ' } 9000 \text { '. } \\
\text { 2. From Step 2, the command returns the requested data object } \\
\text { along with the status word ' } 9000 \text { '. } \\
\text { 3. From Step 4, the authorityInfoAccess extension contains an id- } \\
\text { ad-caIssuers (1.3.6.1.5.5.7.48.2) accessMethod with an } \\
\text { accessLocation of type uniformResourceIdentifier where the } \\
\text { scheme is "http.” }\end{array}$ \\
\hline
\end{tabular}

\subsubsection{8 cRLDistributionPoints Extension}

\begin{tabular}{|c|c|}
\hline Test Assertion & TA-09.03.04.08 \\
\hline Purpose & $\begin{array}{l}\text { Verifies that cRLDistributionPoints extension in the Derived PIV } \\
\text { Credential Issuer's (content signing) certificate contains at least one } \\
\text { URI, either LDAP or HTTP. }\end{array}$ \\
\hline DTR(s) & - $\quad$ DTR-07.03.04.09 \\
\hline Issuer Documentation & None. \\
\hline Precondition(s) & $\begin{array}{l}\text { - A token with the Derived PIV Application is inserted into an } \\
\text { appropriate token reader. } \\
\text { - Suitable drivers have been loaded between the test system and an } \\
\text { instance of the reader. } \\
\text { - A Security Object is present within the Derived PIV Application. }\end{array}$ \\
\hline Test Scenario & 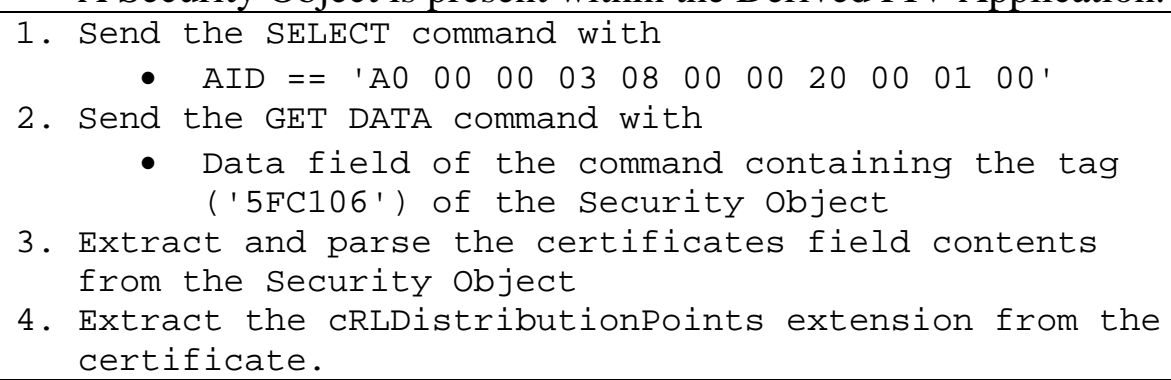 \\
\hline Expected Result(s) & $\begin{array}{l}\text { 1. From Step 1, the command returns the application property } \\
\text { template with the status word ' } 9000 \text { '. }\end{array}$ \\
\hline
\end{tabular}


2. From Step 2, the command returns the requested data object along with the status word ' 9000 '.

3. From Step 4, a URI with either the "LDAP" or "HTTP" scheme that can be used to access CRL information is present. 


\section{Appendix A-Testing of Derived PIV Credentials on Embedded Tokens}

Embedded hardware tokens are not removable from the mobile device, but may be accessed by software using the underlying cryptographic interface of the mobile device. Since these tokens are built into the mobile device, they do not require an application interface definition to enable communication between the token and the mobile device native environment. Nevertheless, embedded tokens can be tested for the service they provide. Two types of testing are described below:

\section{A.1 Functional Testing}

In order to ensure that an embedded Derived PIV Credential follows the specification, test entities may develop test assertions to test these credentials within their operating environment. For example, in order to determine if a Derived PIV Authentication certificate and associated private key on the mobile device can be used for authentication, a test entity may set up an TLSenabled test website and test whether a mobile device with an embedded Derived PIV Authentication certificate can successfully authenticate to the site. Similarly, for testing digital signature and encryption capabilities, a native email client on a mobile device may be setup to sign or decrypt Secure/Multipurpose Internet Mail Extensions (S/MIME) messages and the results reviewed to determine suitable functionality.

\section{A.2 Data Model Testing}

In order to perform data model conformance testing, test entities need to obtain the certificates (i.e., Derived PIV Authentication, digital signature, and key management). Methods for obtaining the certificates include, but are not limited to, (i) performing a functional test and acquiring the certificate by means of that test, (ii) using vendor-specific interface commands to extract the certificates, or (iii) requesting the certificates from the issuer directly.

Once the certificates have been obtain, test entities can follow (as appropriate) the test assertions from Section 9.3 to verify that the certificates conform to the appropriate profiles. 


\section{Appendix B-Acronyms}

API

BER

CMS

CRL

DTR

ECDH

ECDSA

HSPD

HTTP

ICCD

IUT

NIST

OSCP

OID

PC

PIV

PKI

PSS

PUK

RSA

S/MIME

SD

SHA

SIM

SSP

TA

TLV

USB

UICC

URI

URL
Application Programming Interface

Basic Encode Rules

Cryptographic Message Syntax

Certificate Revocation List

Derived Test Requirement

Elliptic Curve Diffie-Hellman

Elliptic Curve Digital Signature Algorithm

Homeland Security Presidential Directive

Hypertext Transfer Protocol

Integrated Circuit(s) Card Devices

Implementation Under Test

National Institute of Standards and Technology

Online Certificate Status Protocol

Object Identifier

Personal Computer

Personal Identity Verification

Public Key Infrastructure

Probabilistic Signature Scheme

Password Unblocking Key

Rivest Shamir Adleman

Secure/Multipurpose Internet Mail Extensions

Secure Digital

Secure Hash Algorithm

Subscriber Identity Module

Shared Service Provider

Test Assertion

Tag-Length-Value

Universal Serial Bus

Universal Integrated Circuit Cards

Uniform Resource Identifier

Uniform Resource Locator 


\section{Appendix C-Glossary of Terms}

Application A part of the application layer in the Open Systems Interconnection Protocol Reference model that is used for communication between two separate Data Unit device's applications. In the context of smart cards, an APDU is the communication unit between a smart card reader and a smart card. The structure of the APDU is defined by [ISO7816-4].

Derived PIV A standardized application residing on a cryptographic token that hosts a Application Derived PIV Credential and associated mandatory and optional elements.

Derived PIV An X.509 Derived PIV Authentication certificate, which is issued in Credential accordance with the requirements specified in [SP800-157], where the PIV Authentication certificate on the Applicant's PIV Card serves as the original credential. The Derived PIV Credential is an additional common identity credential under [HSPD12] and [FIPS201] that is issued by a federal department or agency and that is used with mobile devices

All other significant technical terms used within this document are defined in other key documents including [FIPS201], [SP800-63], and [SP800-73]. 


\section{Appendix D-References}

[FIPS140]

[FIPS201]

[GPSE]

[HSPD12]

[ICCDSPEC]

[ISO7816-4]

[PROF]

[RFC2585]
National Institute of Standards and Technology, Security Requirements for Cryptographic Modules, Federal Information Processing Standards (FIPS) Publication 140-2, May 2001 (including change notices through 12/3/02). http://csrc.nist.gov/publications/fips/fips140-2/fips1402.pdf [accessed 6/3/16].

National Institute of Standards and Technology, Personal Identity Verification (PIV) of Federal Employees and Contractors, Federal Information Processing Standards (FIPS) Publication 201-2, August 2013. http://dx.doi.org/10.6028/NIST.FIPS.201-2.

GlobalPlatform, GlobalPlatform Card Secure Element Configuration v1.0, October 2012. http://www.globalplatform.org/specificationscard.asp [accessed 6/3/16].

Homeland Security Presidential Directive-12, Policies for a Common Identification Standard for Federal Employees and Contractors, August 27, 2004. http://www.dhs.gov/homeland-security-presidential-directive-12 [accessed 6/3/16].

Universal Serial Bus Device Class: Smart Card ICCD Specification for USB Integrated Circuit(s) Card Devices, Revision 1.0, April 22, 2005. http://www.usb.org/developers/docs/devclass_docs/DWG_SmartCard_USB-ICC_ICCD_rev10.pdf [accessed 6/3/16].

International Organization for Standardization/International Electrotechnical Commission (ISO/IEC), Identification cards - Integrated circuit cards - Part 4: Organization, security and commands for interchange, ISO/IEC 7816-4:2013, 2013.

http://www.iso.org/iso/home/store/catalogue_ics/catalogue_detail_ics.htm? csnumber $=54550$ [accessed 6/3/16].

Federal PKI Policy Authority Shared Service Provider Working Group, X.509 Certificate and Certificate Revocation List (CRL) Extensions Profile for the Shared Service Providers (SSP) Program, Version 1.7, May 5, 2015.

https://www.idmanagement.gov/IDM/s/document_detail?Id=kA0t0000000 8ObeCAE [accessed 6/3/16].

R. Housley and P. Hoffman, Internet X.509 Public Key Infrastructure Operational Protocols: FTP and HTTP, Internet Engineering Task Force (IETF) Network Working Group Request for Comments (RFC) 2585, May 1999. http://dx.doi.org/10.17487/RFC2585. 
[RFC3370] R. Housley, Cryptographic Message Syntax (CMS) Algorithms, Internet Engineering Task Force (IETF) Network Working Group Request for Comments (RFC) 3370, August 2002. http://dx.doi.org/10.17487/RFC3370.

[RFC4122] P. Leach, M. Mealling, and R. Salz, A Universally Unique IDentifier (UUID) URN Namespace, Internet Engineering Task Force (IETF) Network Working Group Request for Comments (RFC) 4122, July 2005. http://dx.doi.org/10.17487/RFC4122.

[RFC5652] R. Housley, Cryptographic Message Syntax (CMS), Internet Engineering Task Force (IETF) Network Working Group Request for Comments (RFC) 5652, September 2009. http://dx.doi.org/10.17487/RFC5652.

[RFC5751] B. Ramsdell and S. Turner, Secure/Multipurpose Internet Mail Extensions (S/MIME) Version 3.2 Message Specification, Internet Engineering Task Force (IETF) Request for Comments (RFC) 5751, January 2010. http://dx.doi.org/10.17487/RFC5751.

[SEC1] Certicom Research, SEC 1: Elliptic Curve Cryptography, Version 1.0, Standards for Efficient Cryptography, September 2000. http://www.secg.org/SEC1-Ver-1.0.pdf [accessed 6/3/16].

[SP800-56A] National Institute of Standards and Technology, Recommendation for PairWise Key Establishment Schemes Using Discrete Logarithm Cryptography, NIST Special Publication (SP) 800-56A Revision 2, May 2013. http://dx.doi.org/10.6028/NIST.SP.800-56Ar2.

[SP800-63] National Institute of Standards and Technology, Electronic Authentication Guideline, NIST Special Publication (SP) 800-63-2, August 2013. http://dx.doi.org/10.6028/NIST.SP.800-63-2.

[SP800-73] National Institute of Standards and Technology, Interfaces for Personal Identity Verification, NIST Special Publication (SP) 800-73-4, May 2015 (includes updates as of 2/8/16).

http://dx.doi.org/10.6028/NIST.SP.800-73-4.

[SP800-78] National Institute of Standards and Technology, Cryptographic Algorithms and Key Sizes for Personal Identity Verification, NIST Special Publication (SP) 800-78-4, May 2015. http://dx.doi.org/10.6028/NIST.SP.800-78-4.

[SP800-79] National Institute of Standards and Technology, Guidelines for the Authorization of Personal Identity Verification Card Issuers (PCI) and Derived PIV Credential Issuers (DPCI), NIST Special Publication (SP) 800-79-2, July 2015. http://dx.doi.org/10.6028/NIST.SP.800-79-2. 
[SP800-96] National Institute of Standards and Technology, PIV Card to Reader Interoperability Guidelines, NIST Special Publication (SP) 800-96, September 2006. http://dx.doi.org/10.6028/NIST.SP.800-96.

[SP800-157] National Institute of Standards and Technology, Guidelines for Derived Personal Identity Verification (PIV) Credentials, NIST Special Publication (SP) 800-157, December 2014. http://dx.doi.org/10.6028/NIST.SP.800$\underline{157 .}$. 